\title{
Structure-Based Design, Synthesis and Biological Evaluation of New Triazolo[1,5- a]Pyrimidine Derivatives as Highly Potent and Orally Active ABCB1 Modulators
}

Shuai Wang ${ }^{a, \#}$, Sai-Qi Wang ${ }^{b, \#}$, Qiu-Xu Teng ${ }^{c, \#}$, Linlin Yang $^{d, \#}$, Zi-Ning Lei ${ }^{c}$, Xiao-Han Yuan ${ }^{a}$, Jun-Feng Huo ${ }^{a}$, Xiao-Bing Chen ${ }^{b}$, Mengru Wang ${ }^{d}$, Bin Yu ${ }^{a,{ }^{*}}$, Zhe-Sheng Chen ${ }^{c,}{ }^{*}$, and Hong-Min $\operatorname{Liu}^{a, *}$

a School of Pharmaceutical Sciences \& Key Laboratory of Advanced Drug Preparation Technologies, Ministry of Education, Zhengzhou University, Zhengzhou 450001, China;

${ }^{b}$ Department of Oncology, The Affiliated Cancer Hospital of Zhengzhou University, Henan Cancer Hospital, Henan cancer institute, Zhengzhou 450008, China;

${ }^{\mathrm{c}}$ College of Pharmacy and Health Sciences, St. John's University, Queens, NY, 11439, USA;

d Department of Pharmacology, School of Basic Medical Sciences, Zhengzhou University, Zhengzhou 450001, China;

\section{Corresponding authors:}

Prof.BinYu(yubin@zzu.edu.cn)

Prof. Zhe-Sheng Chen (chenz@stjohns.edu)

Prof. Hong-Min Liu (liuhm@zzu.edu.cn)

\# Shuai Wang, Sai-Qi Wang, Qiu-Xu Teng and Linlin Yang contributed equally to this work.* 


\section{Table of Contents}

1. Cell survival rate under the nontoxic concentration of compounds toward SW620/AD300 cells

2. Cell survival rate under the nontoxic concentration of WS-691 toward selected cancer cells 3. NMR spectra of products

4. LCMS spectra of products

5. HPLC spectra of products 
Table S1. Cell survival rate under the nontoxic concentration of compounds toward SW620/AD300 cells

\begin{tabular}{ccc|ccc}
\hline Compound & $\begin{array}{c}\text { Concentration } \\
(\mu \mathrm{M})\end{array}$ & $\begin{array}{c}\text { Survival Rate } \\
(\%)\end{array}$ & $\begin{array}{c}\text { Compound } \\
\text { WS-36 (5) }\end{array}$ & $\begin{array}{c}\text { Concentration } \\
(\mu \mathrm{M})\end{array}$ & $\begin{array}{c}\text { Survival Rate } \\
(\%)\end{array}$ \\
\hline $\mathbf{6}$ & 20 & $93.25 \pm 3.78$ & $\mathbf{3 1}$ & 20 & $92.96 \pm 5.01$ \\
$\mathbf{7}$ & 20 & $95.34 \pm 2.46$ & $\mathbf{3 2}$ & 15 & $94.23 \pm 9.51$ \\
$\mathbf{8}$ & 20 & $94.02 \pm 2.05$ & $\mathbf{3 3}$ & 20 & $97.91 \pm 3.65$ \\
$\mathbf{9}$ & 20 & $93.41 \pm 2.45$ & $\mathbf{3 4}$ & 20 & $91.46 \pm 2.39$ \\
$\mathbf{1 0}$ & 20 & $91.37 \pm 2.30$ & $\mathbf{3 5}$ & 20 & $90.10 \pm 4.50$ \\
$\mathbf{1 1}$ & 20 & $94.66 \pm 4.33$ & $\mathbf{3 6}$ & 20 & $97.95 \pm 2.79$ \\
$\mathbf{1 2}$ & 20 & $93.01 \pm 4.72$ & $\mathbf{3 7}$ & 20 & $92.10 \pm 4.30$ \\
$\mathbf{1 3}$ & 15 & $91.27 \pm 4.86$ & $\mathbf{3 8}$ & 20 & $95.97 \pm 2.24$ \\
$\mathbf{1 4}$ & 20 & $98.79 \pm 5.99$ & $\mathbf{3 9}$ & 20 & $99.12 \pm 2.22$ \\
$\mathbf{1 5}$ & 15 & $91.49 \pm 3.78$ & $\mathbf{4 0}$ & 20 & $96.21 \pm 4.19$ \\
$\mathbf{1 6}$ & 20 & $91.10 \pm 2.91$ & $\mathbf{4 1}$ & 20 & $93.12 \pm 2.98$ \\
$\mathbf{1 7}$ & 20 & $97.76 \pm 5.02$ & $\mathbf{4 2}$ & 20 & $92.08 \pm 3.95$ \\
$\mathbf{1 8}$ & 20 & $95.52 \pm 2.53$ & $\mathbf{4 3}$ & 20 & $93.66 \pm 7.01$ \\
$\mathbf{1 9}$ & 20 & $90.84 \pm 6.06$ & $\mathbf{4 4}$ & 20 & $91.62 \pm 2.47$ \\
$\mathbf{2 0}$ & 20 & $92.57 \pm 1.38$ & $\mathbf{4 5}$ & 20 & $97.81 \pm 1.69$ \\
$\mathbf{2 1}$ & 20 & $97.86 \pm 1.82$ & $\mathbf{4 6}$ & 20 & $95.50 \pm 5.37$ \\
$\mathbf{2 2}$ & 20 & $90.90 \pm 2.55$ & $\mathbf{4 7}$ & 20 & $94.56 \pm 1.86$ \\
$\mathbf{2 3}$ & 20 & $98.86 \pm 2.34$ & $\mathbf{4 8}$ & 20 & $92.35 \pm 3.90$ \\
$\mathbf{2 4}$ & 20 & $94.98 \pm 2.86$ & $\mathbf{4 9}$ & 20 & $96.63 \pm 3.22$ \\
$\mathbf{2 5}$ & 20 & $93.80 \pm 1.94$ & $\mathbf{5 0}$ & 20 & $94.35 \pm 2.61$ \\
$\mathbf{2 6}$ & 20 & $92.36 \pm 2.16$ & $\mathbf{5 1}$ & 20 & $92.46 \pm 2.66$ \\
$\mathbf{W S - 6 9 1}(\mathbf{2 7})$ & 20 & $96.10 \pm 4.71$ & $\mathbf{5 2}$ & 20 & $97.39 \pm 2.50$ \\
$\mathbf{2 8}$ & 20 & $91.12 \pm 2.22$ & $\mathbf{5 3}$ & 20 & $90.74 \pm 3.33$ \\
$\mathbf{2 9}$ & 20 & $97.40 \pm 5.66$ & $\mathbf{5 4}$ & 15 & $96.80 \pm 3.46$ \\
$\mathbf{3 0}$ & 20 & $95.59 \pm 3.12$ & $\mathbf{5 5}$ & 20 & $96.60 \pm 5.66$ \\
& & & & \\
\hline
\end{tabular}


Table S2. Cell survival rate under the nontoxic concentration of WS-691 toward selected cancer cells

\begin{tabular}{ccc}
\hline \multirow{2}{*}{ Cells } & \multicolumn{2}{c}{ Survival Rate (\%) } \\
\cline { 2 - 3 } & $\begin{array}{c}\text { Concentration } \\
(10 \mu \mathrm{M})\end{array}$ & $\begin{array}{c}\text { Concentration } \\
(20 \mu \mathrm{M})\end{array}$ \\
\hline SW620 & $98.79 \pm 8.32$ & $93.53 \pm 4.32$ \\
SW620/AD300 & $95.51 \pm 3.25$ & $91.12 \pm 2.22$ \\
KB-31 & $95.27 \pm 4.12$ & $90.26 \pm 1.35$ \\
KB-C2 & $96.62 \pm 2.29$ & $93.42 \pm 3.56$ \\
HEK293/pcDNA3.1 & $97.52 \pm 3.14$ & $90.13 \pm 1.51$ \\
HEK293/ABCB1 & $98.36 \pm 2.58$ & $93.63 \pm 1.96$ \\
\hline
\end{tabular}



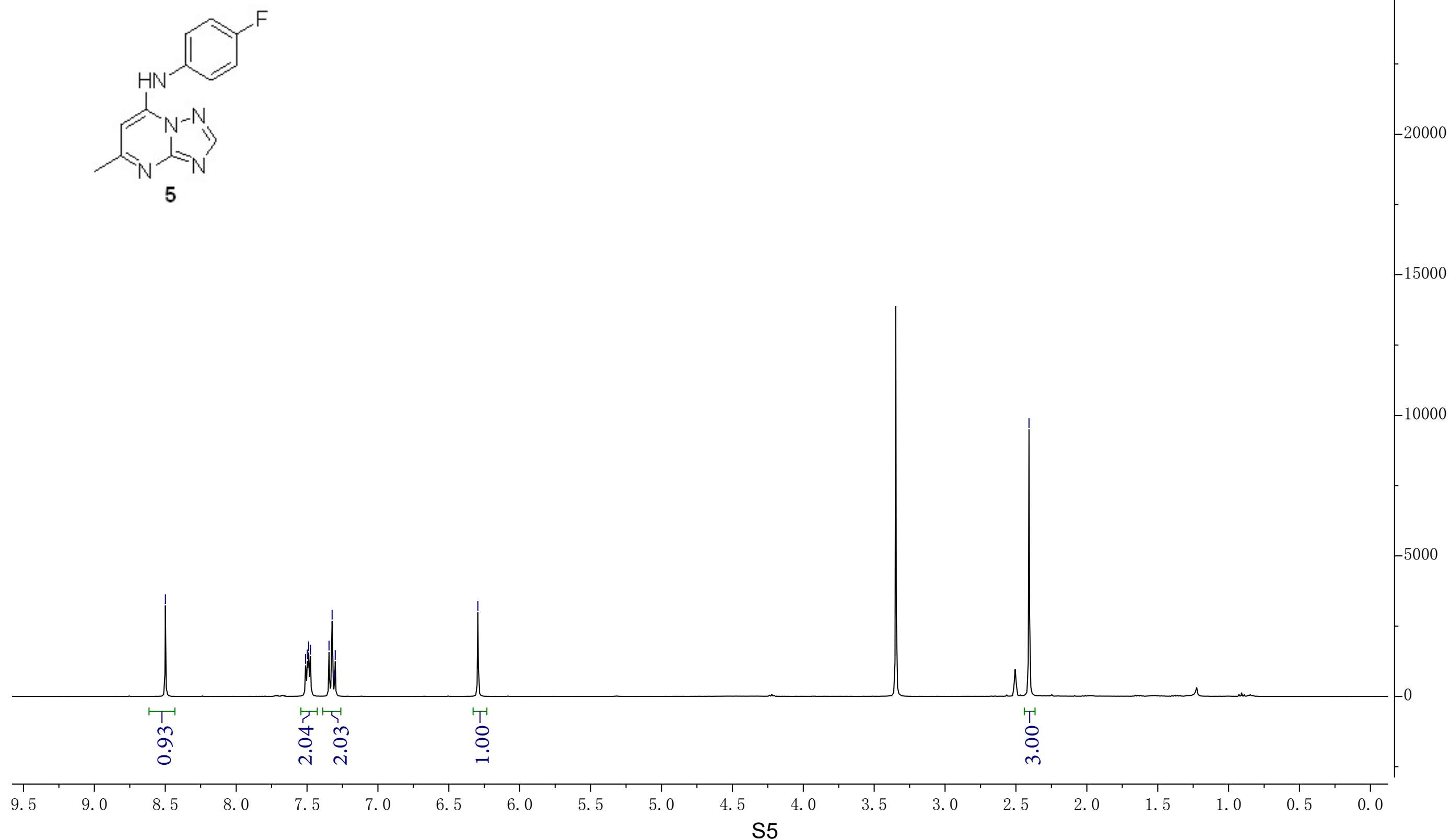


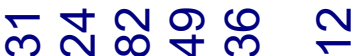

श 50

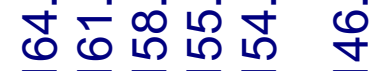

$\infty$

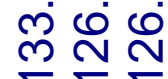

ํㅜㄷ

๑

Гॅ

운 눈
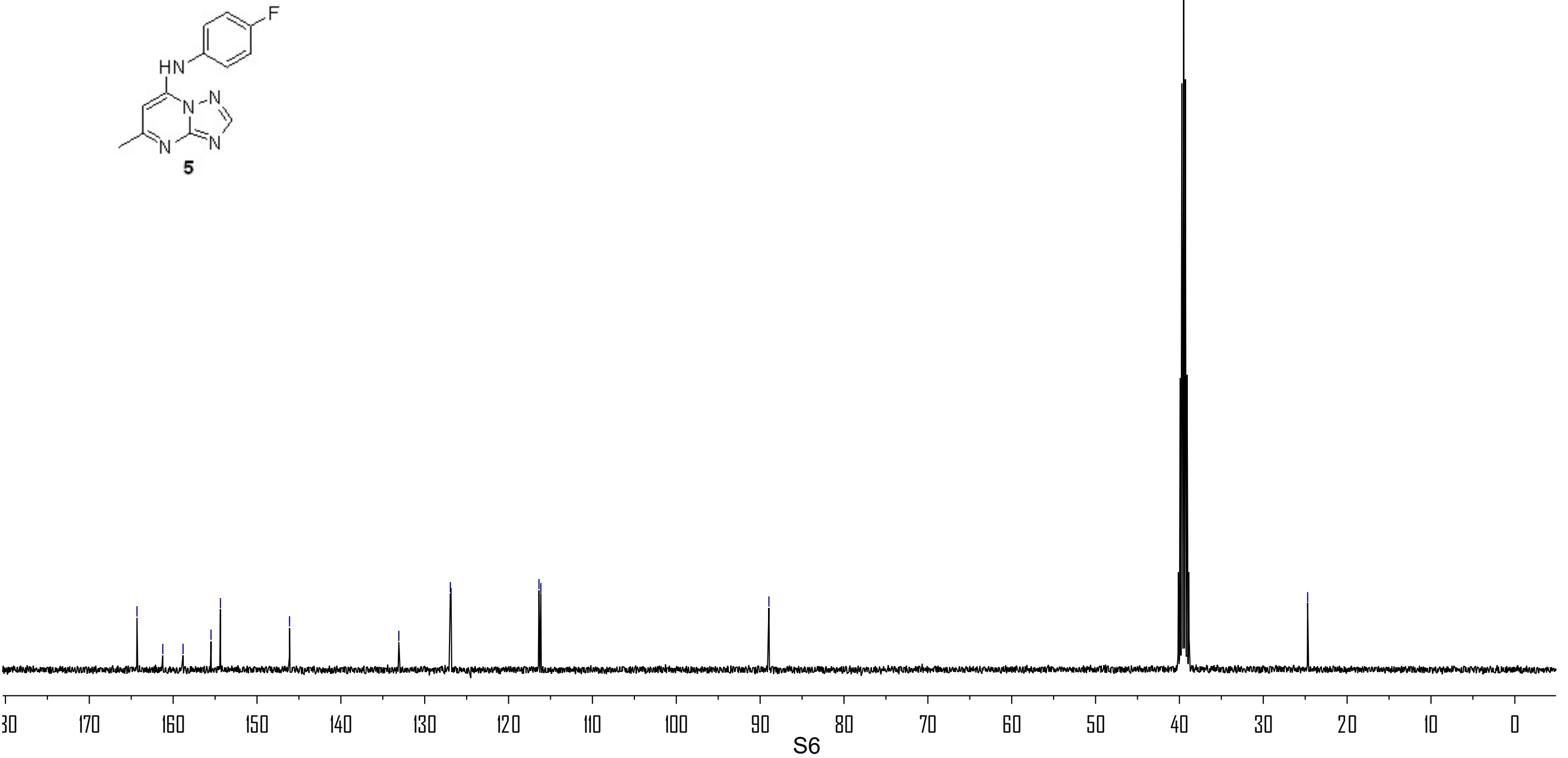


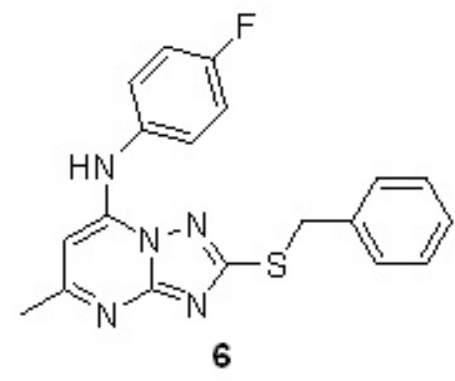



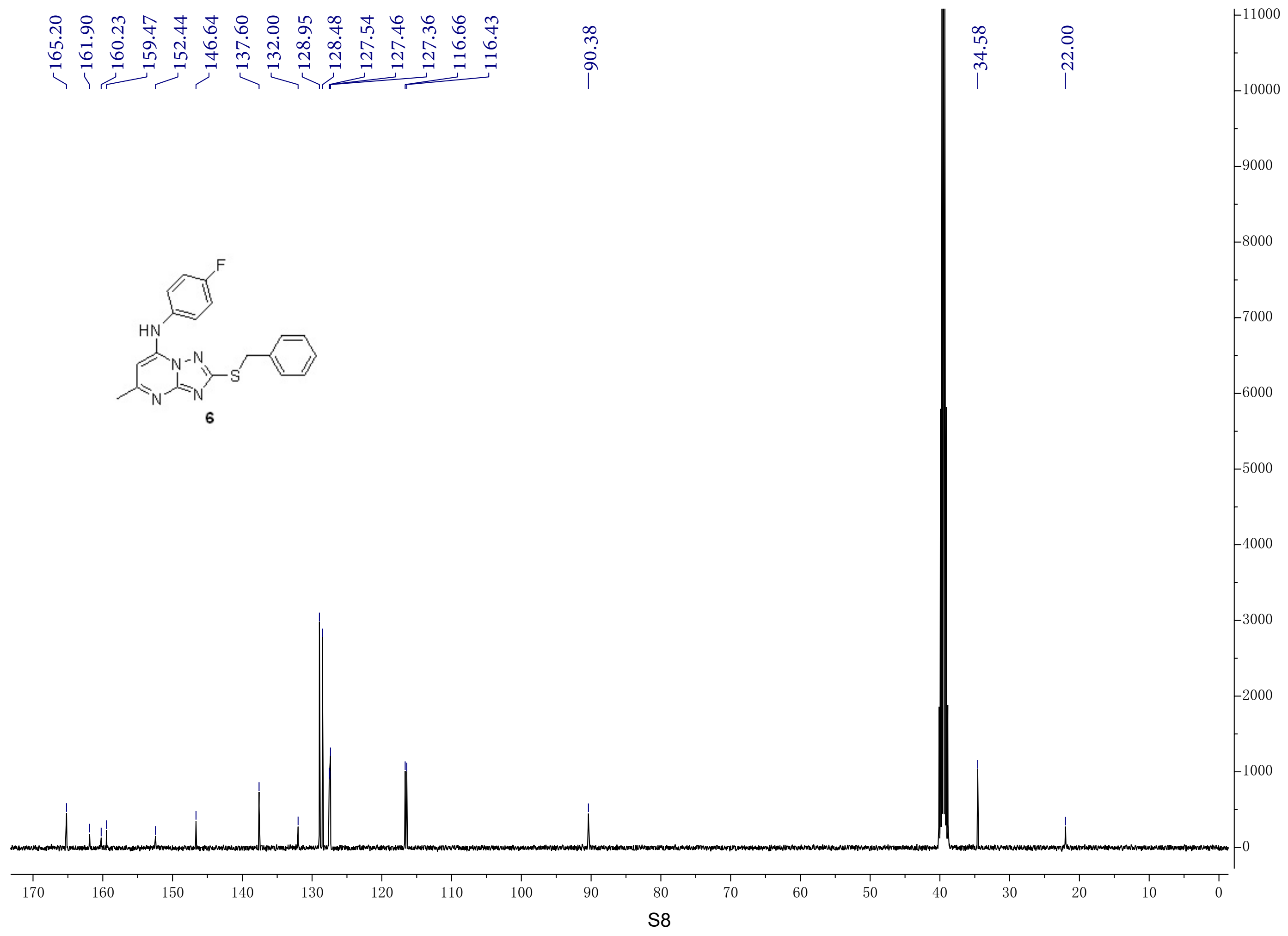

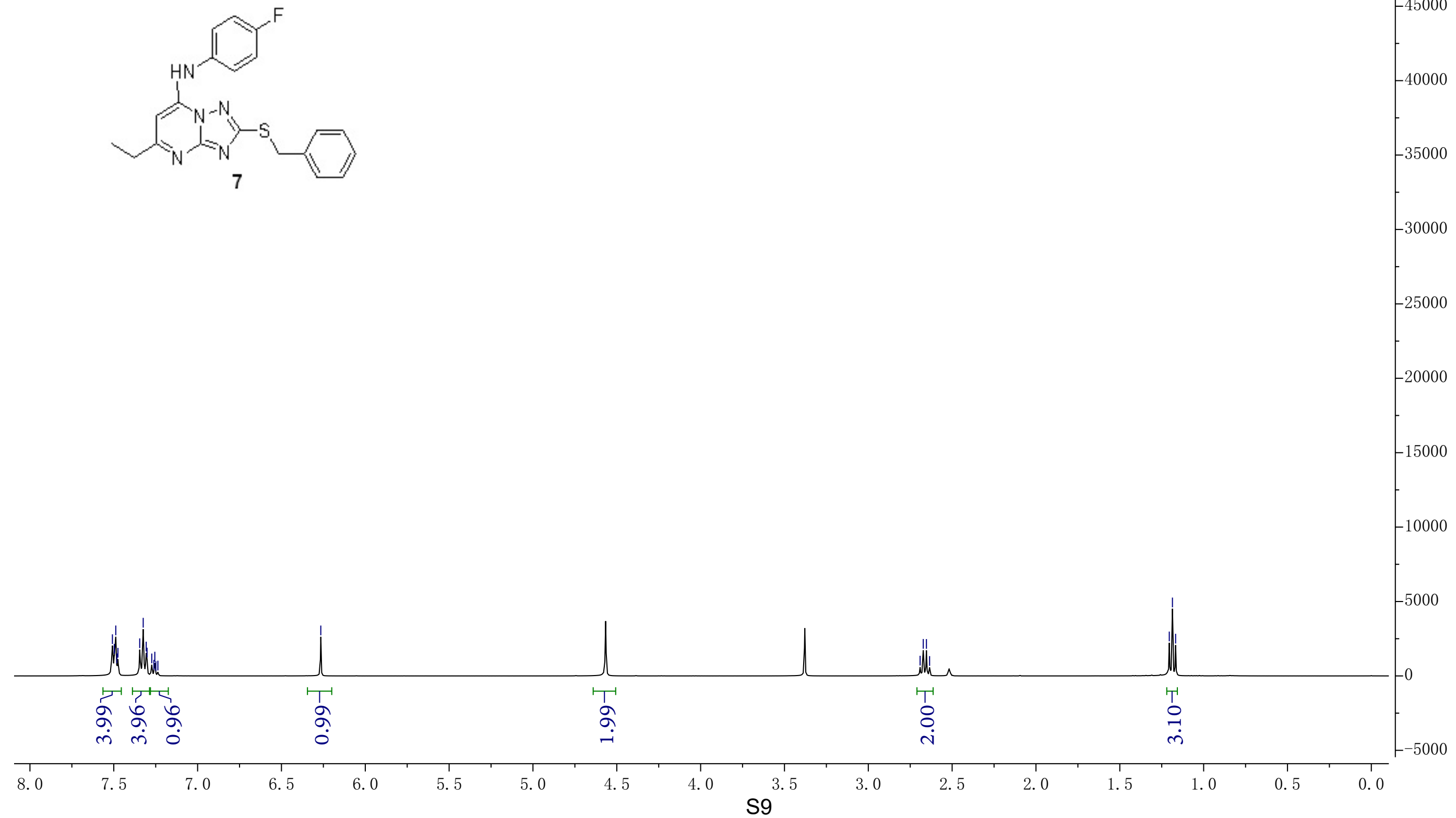

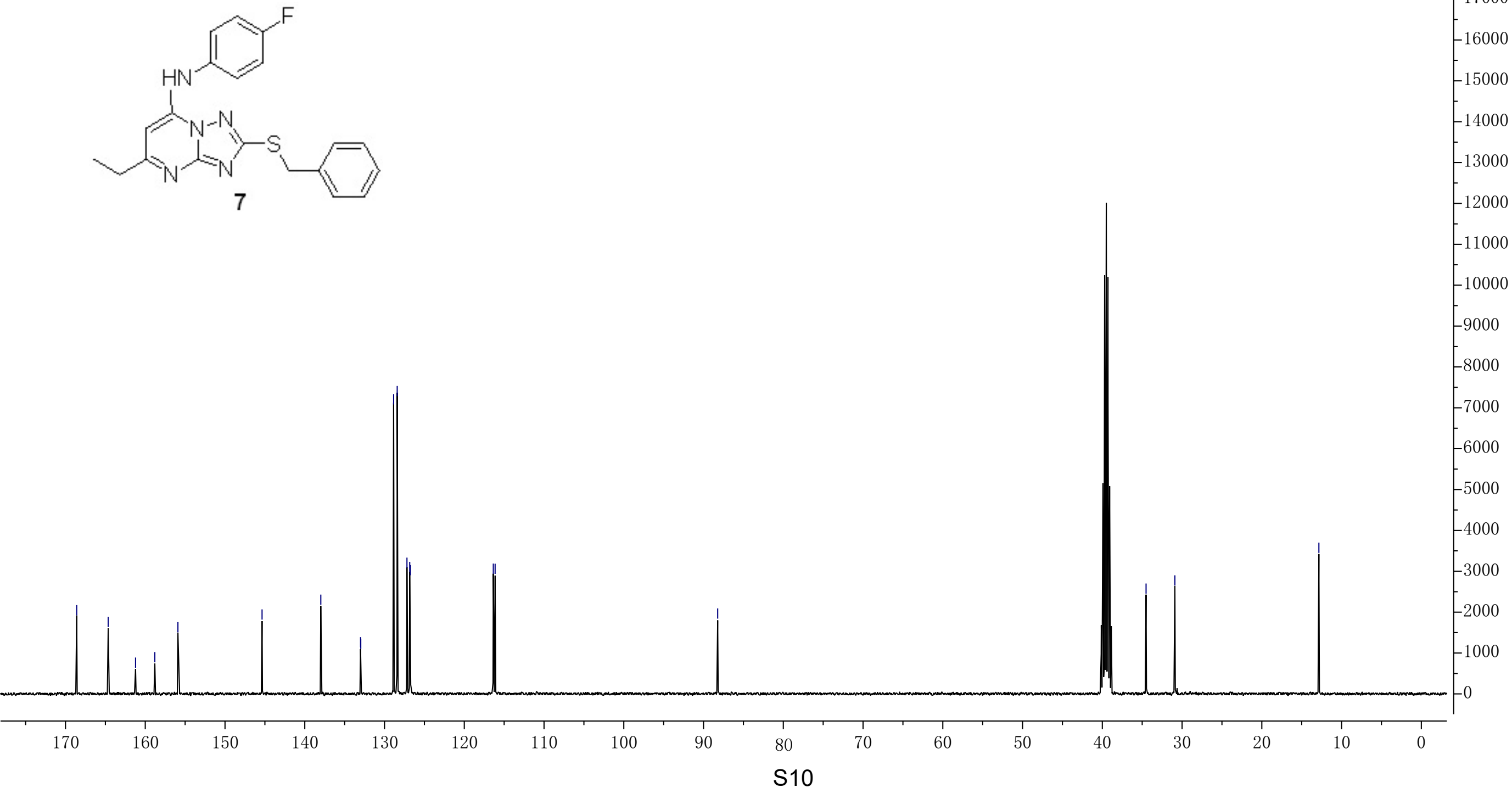

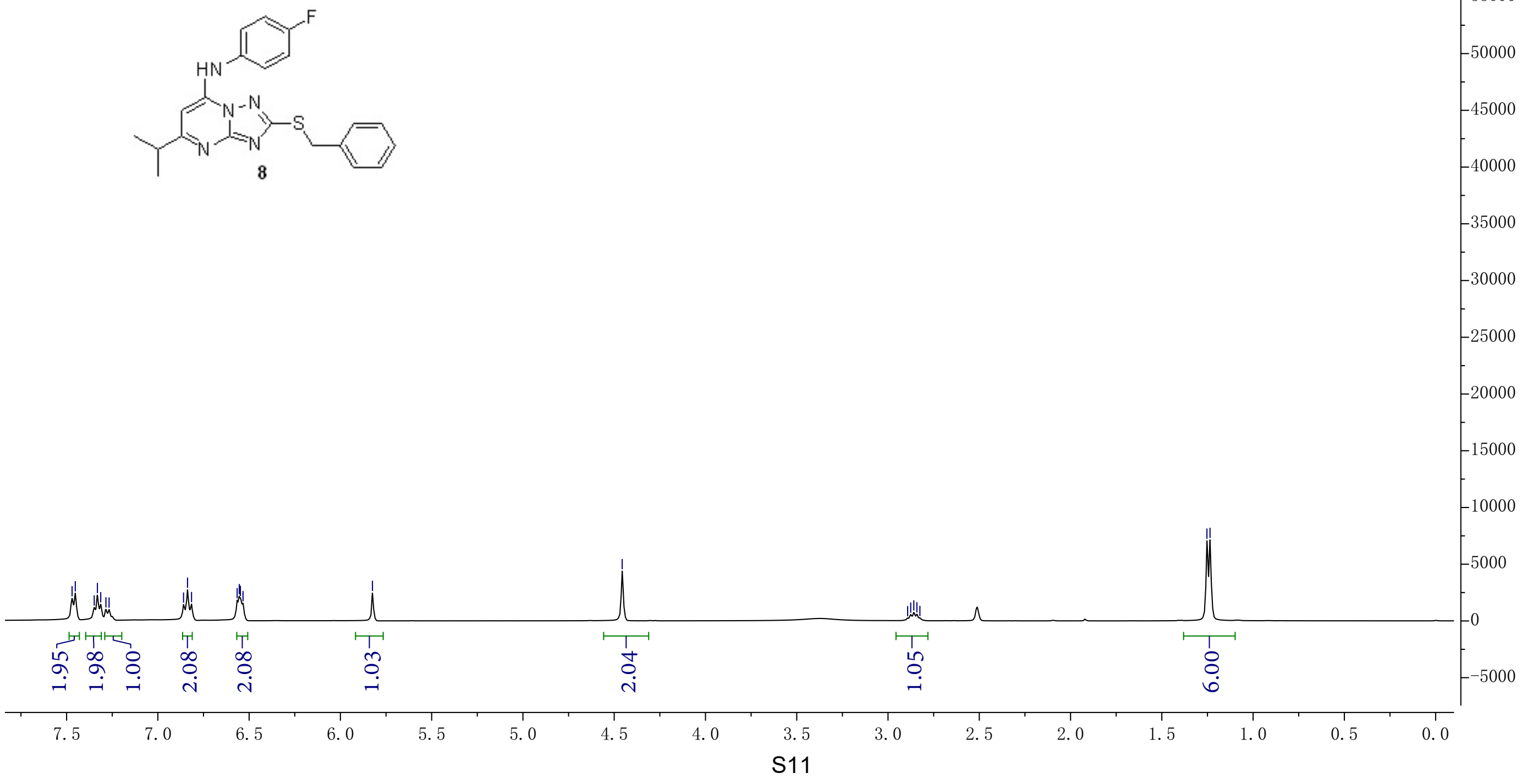

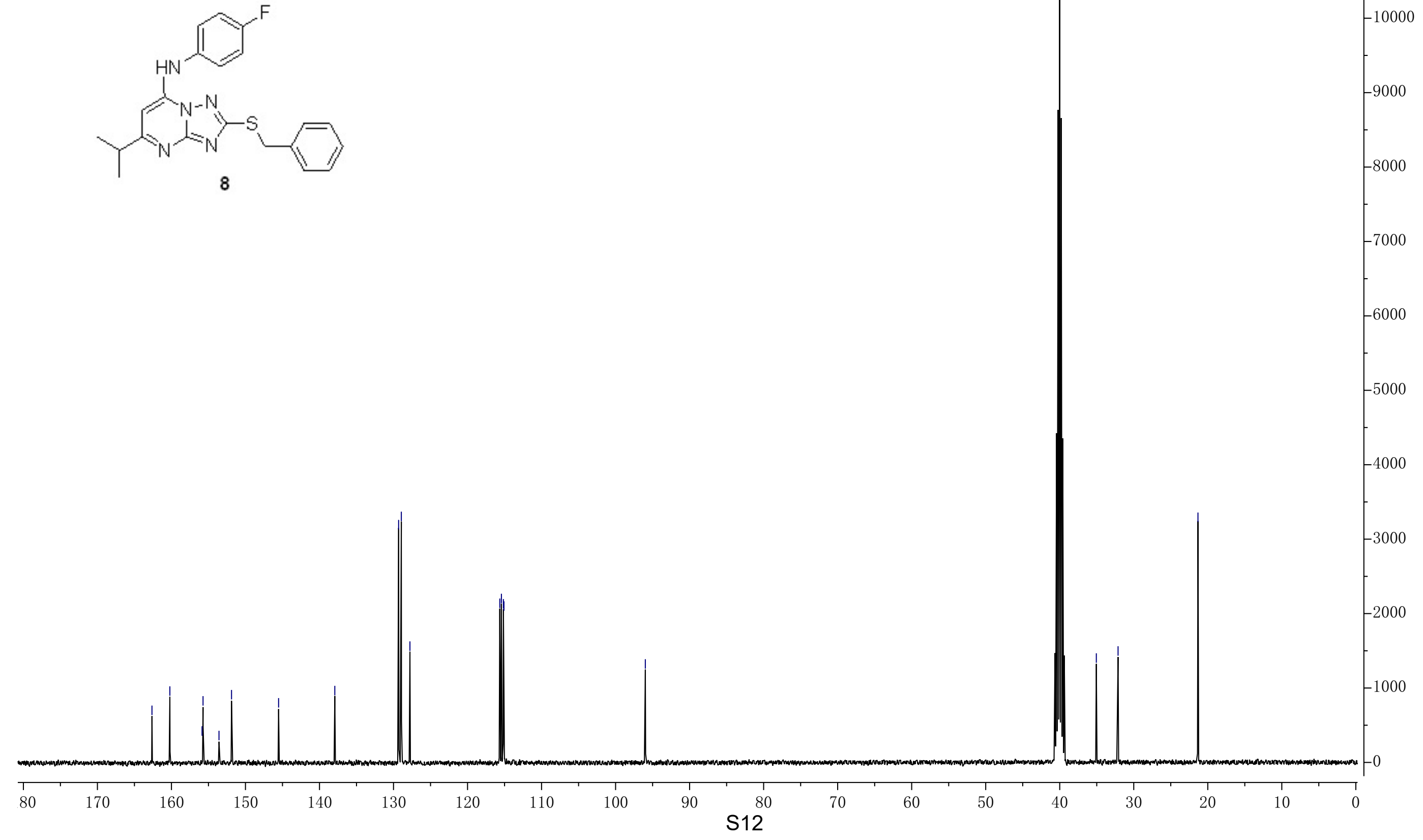

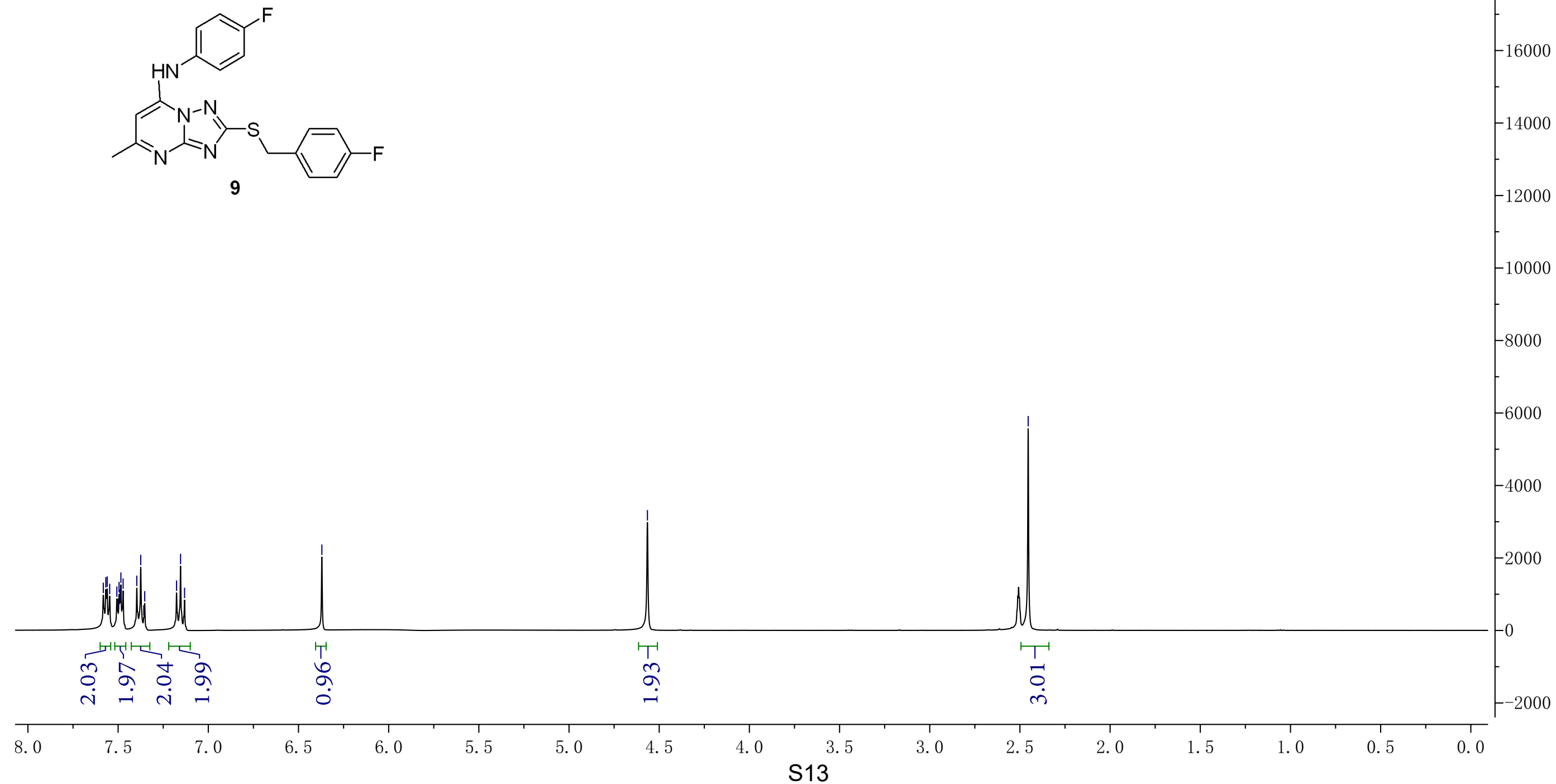


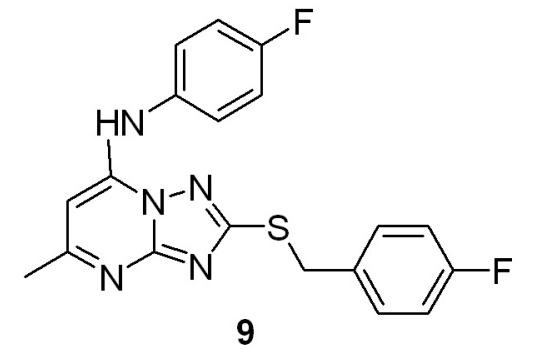



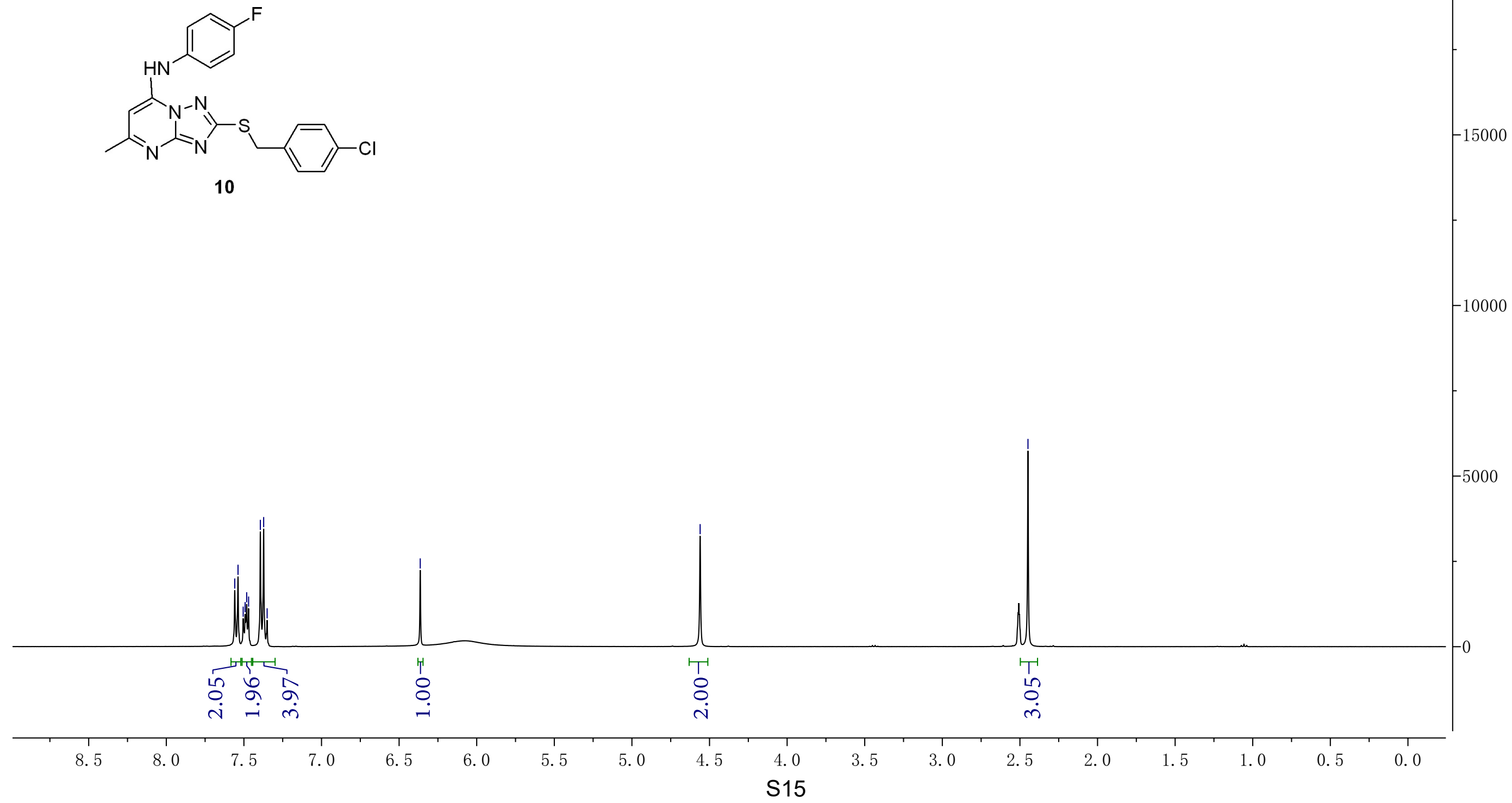


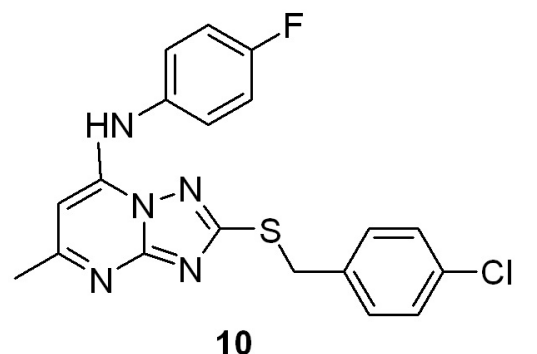



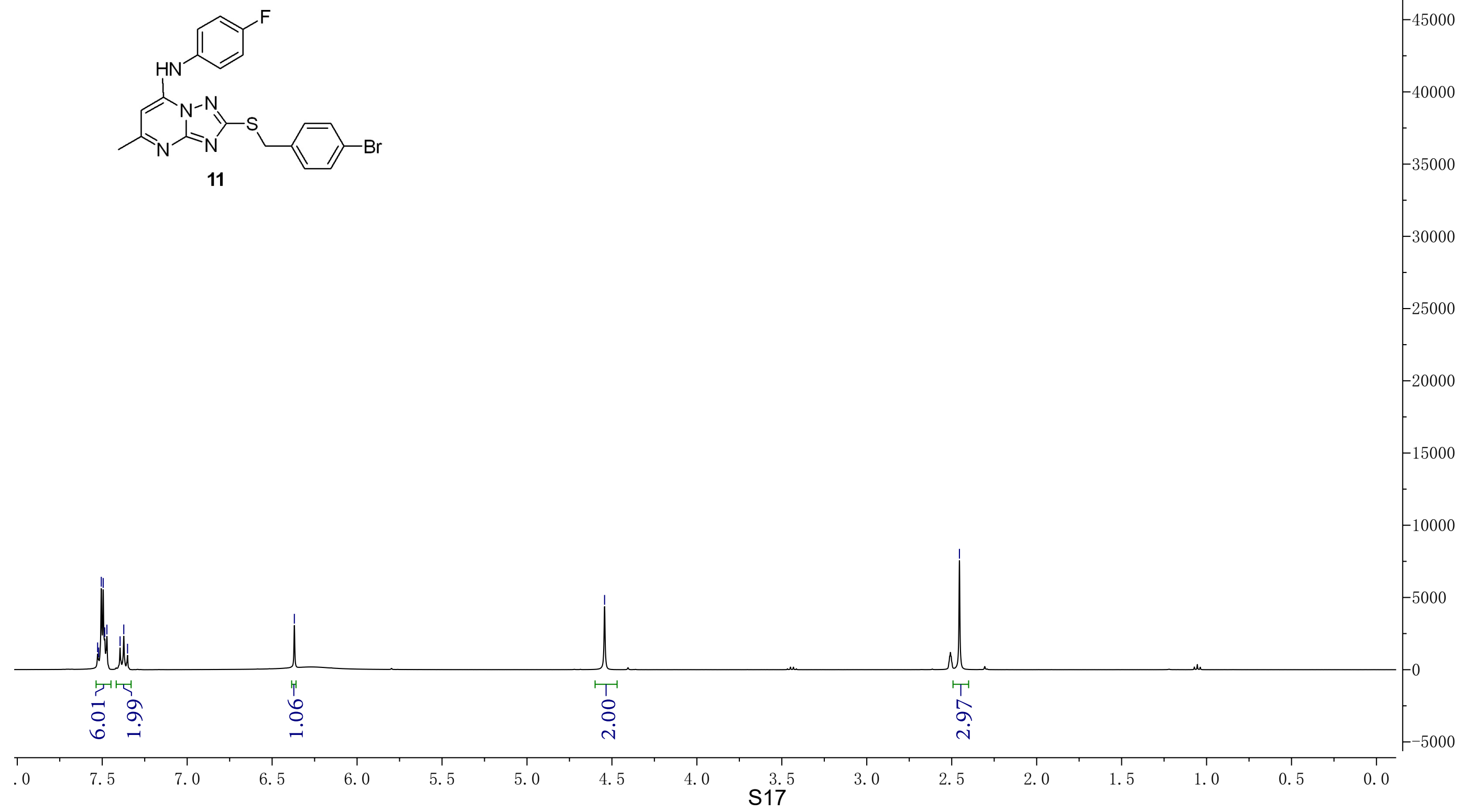

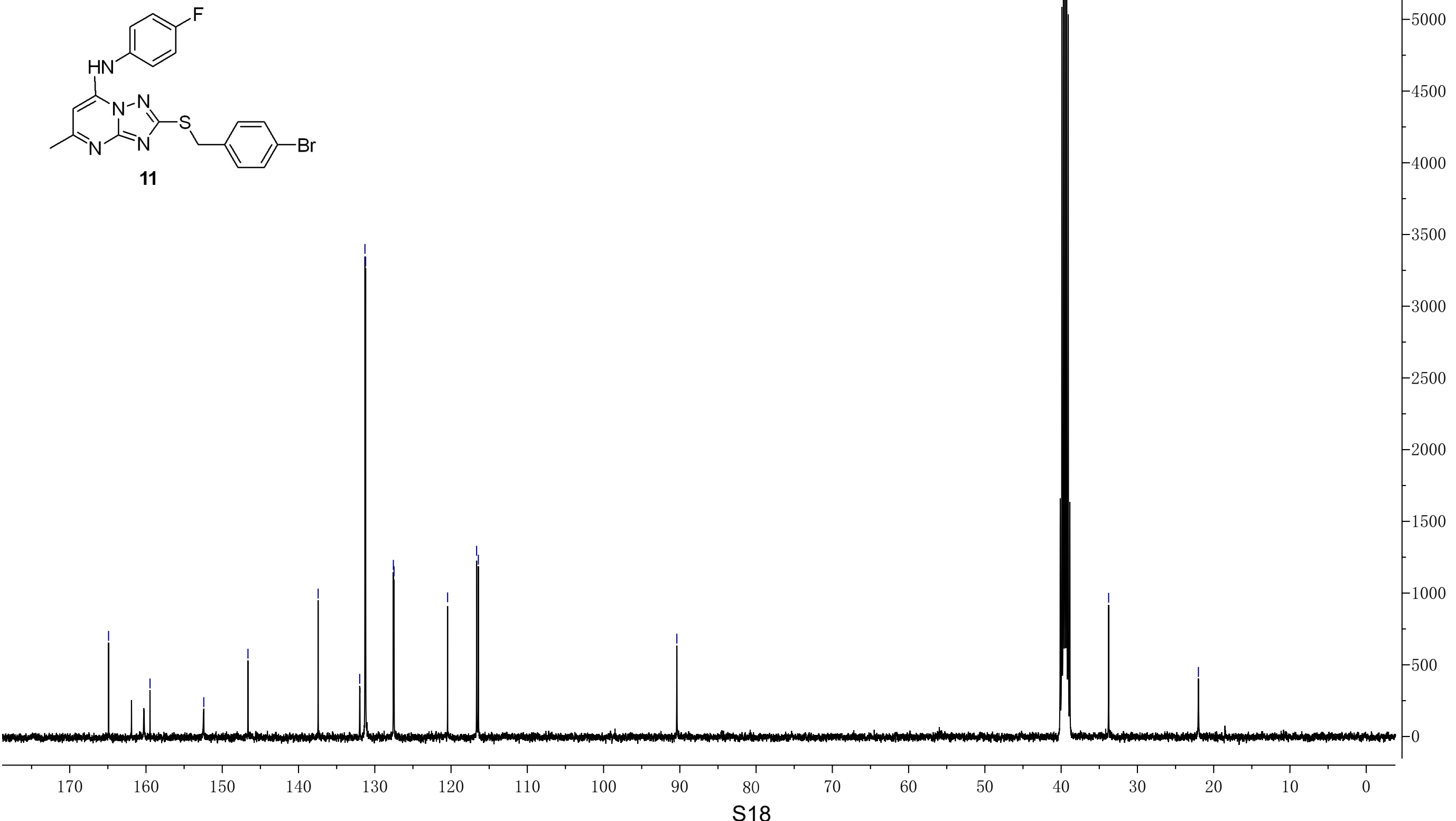

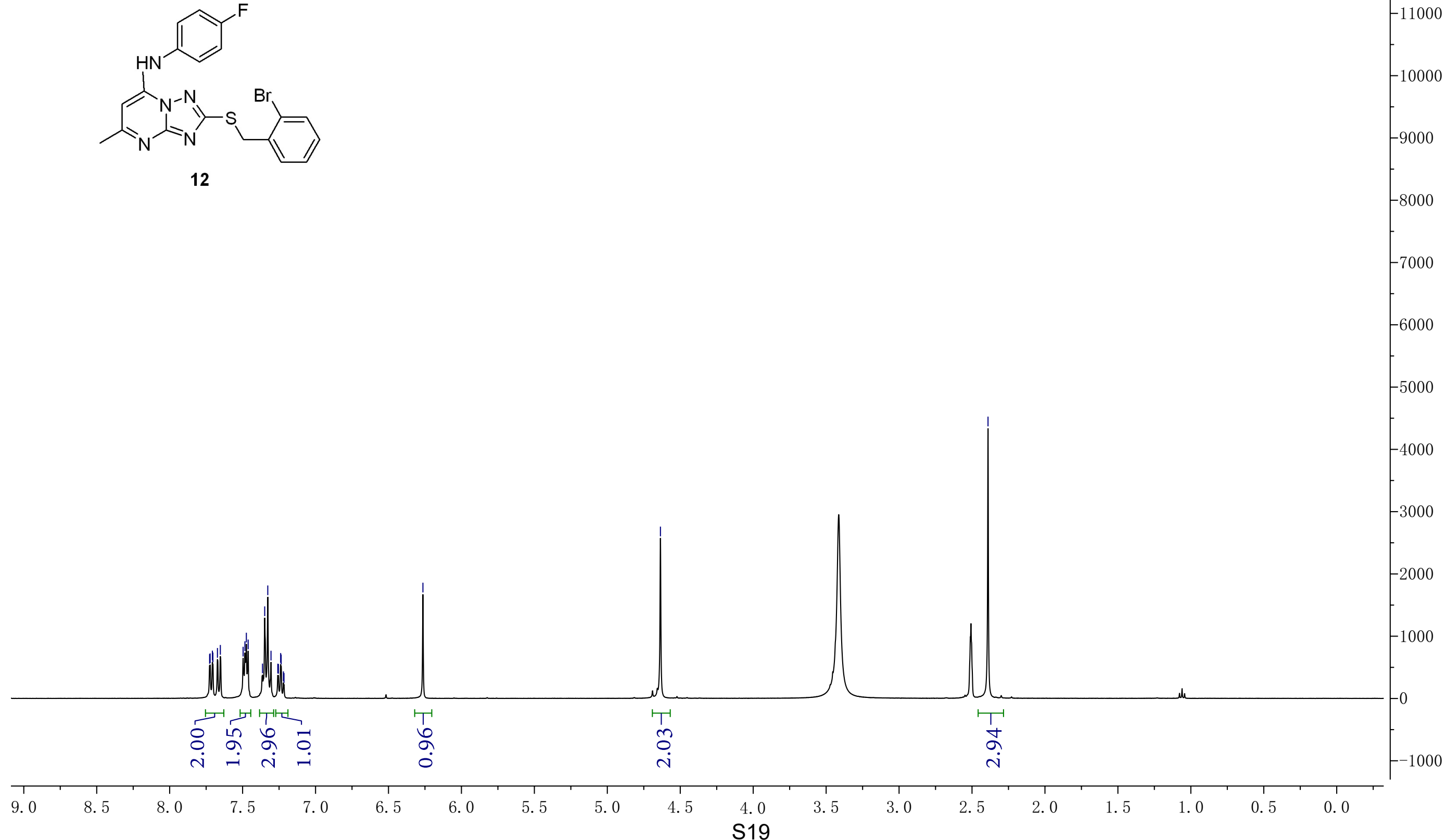

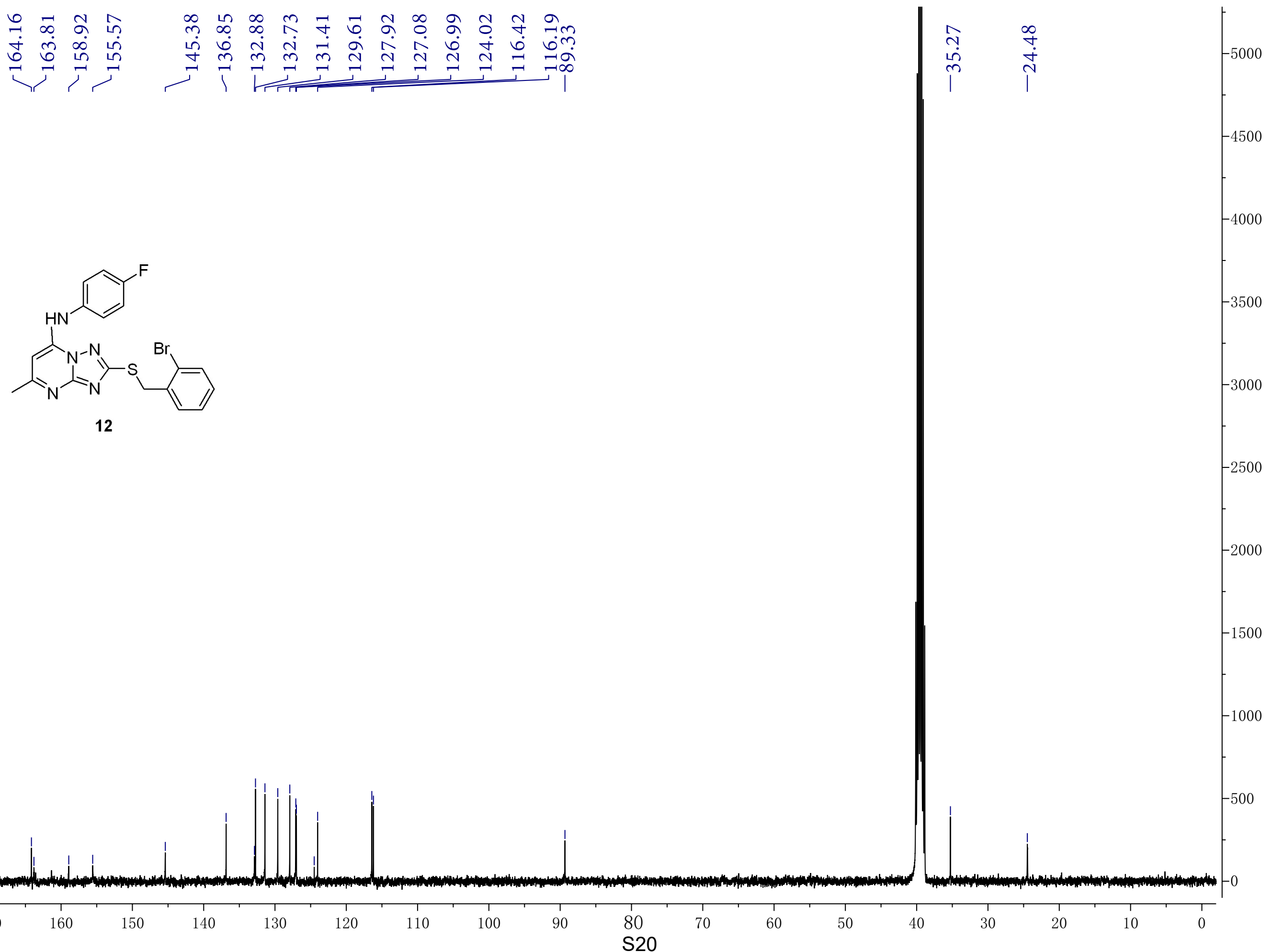

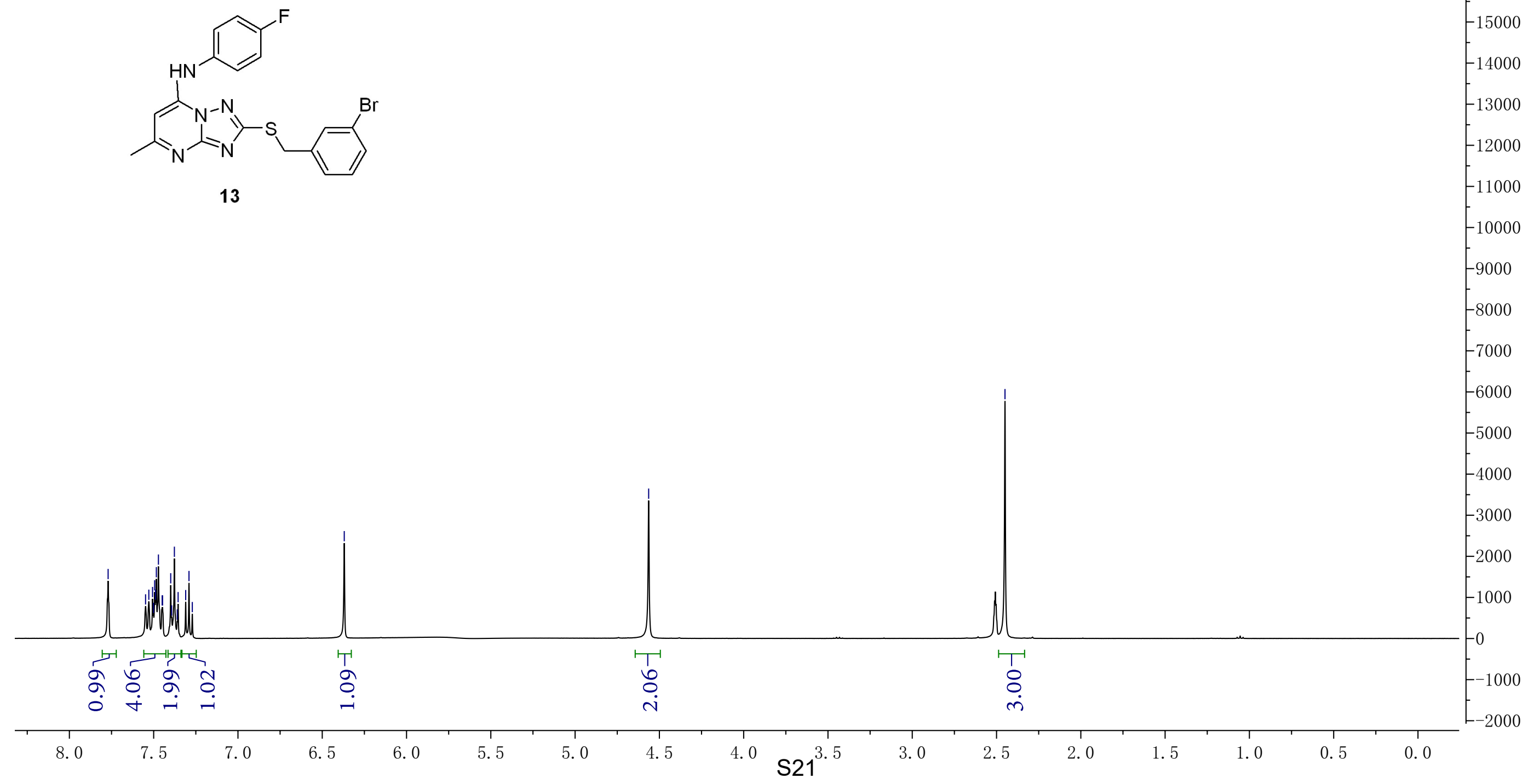

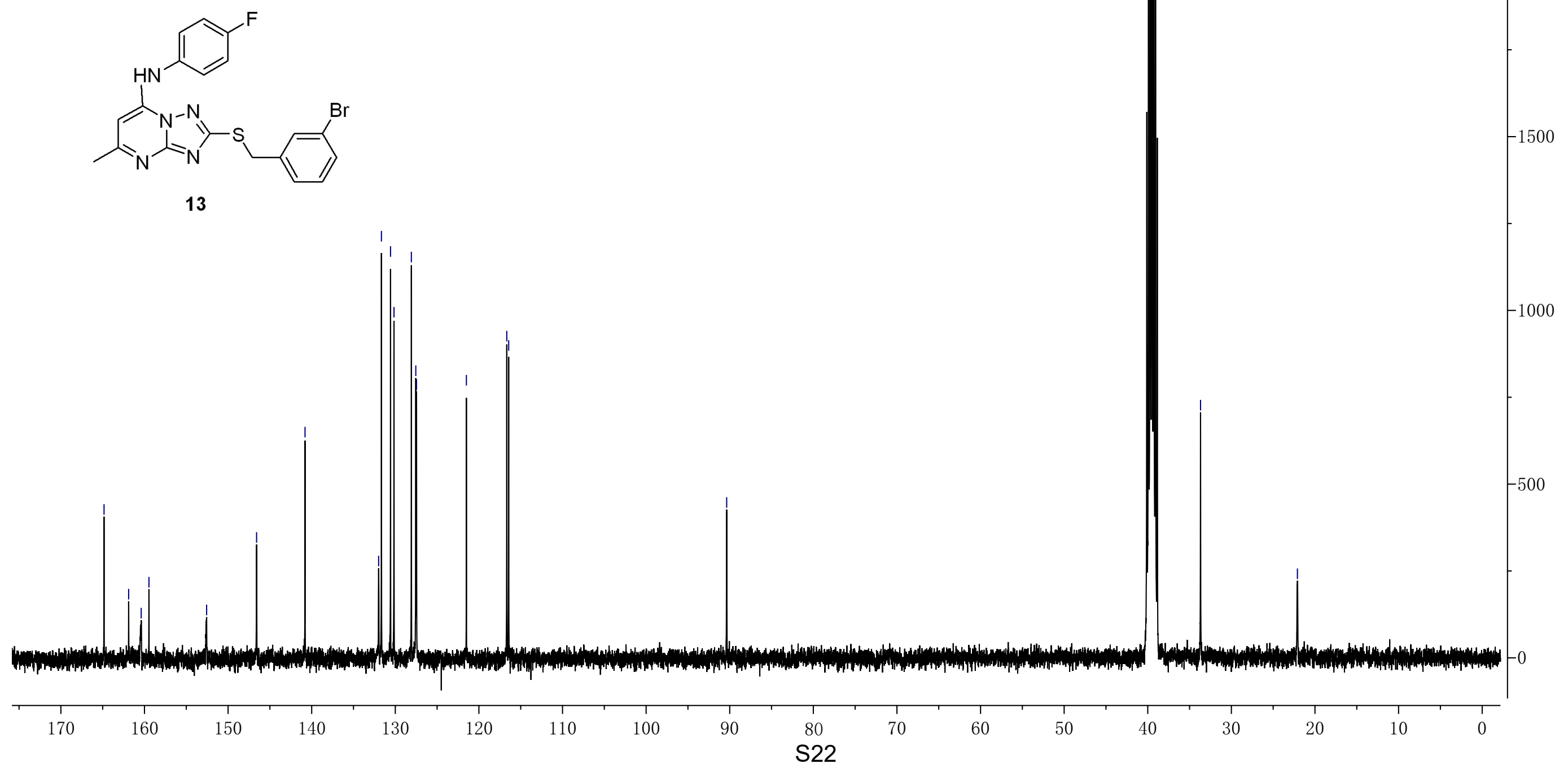

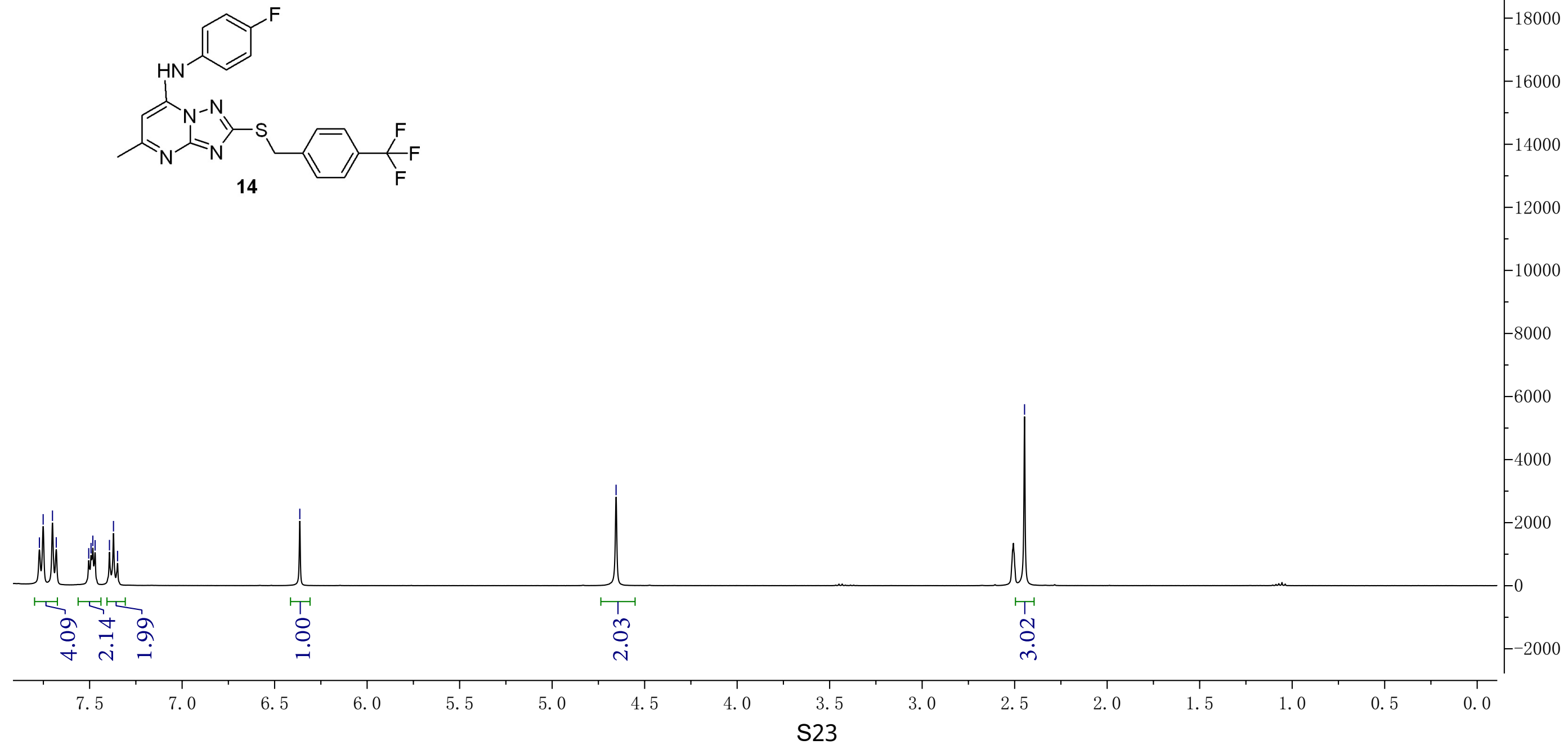


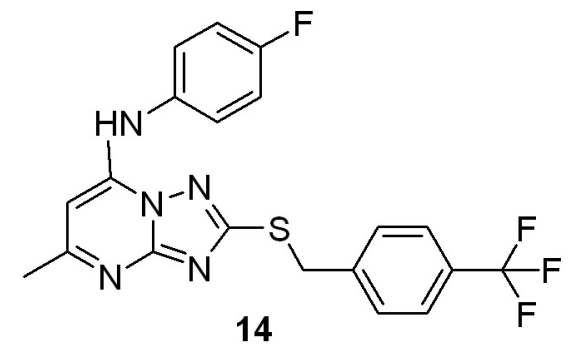



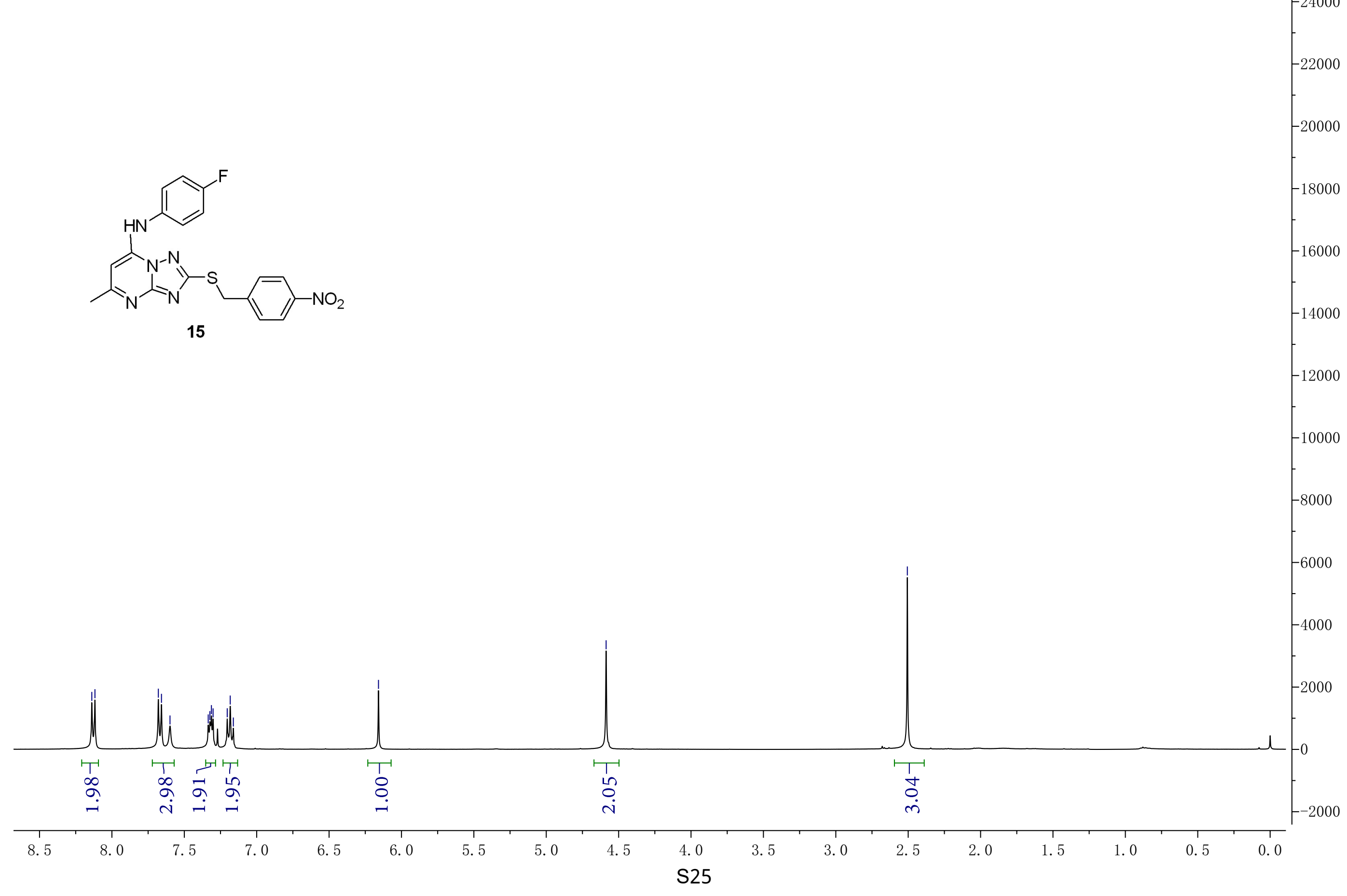

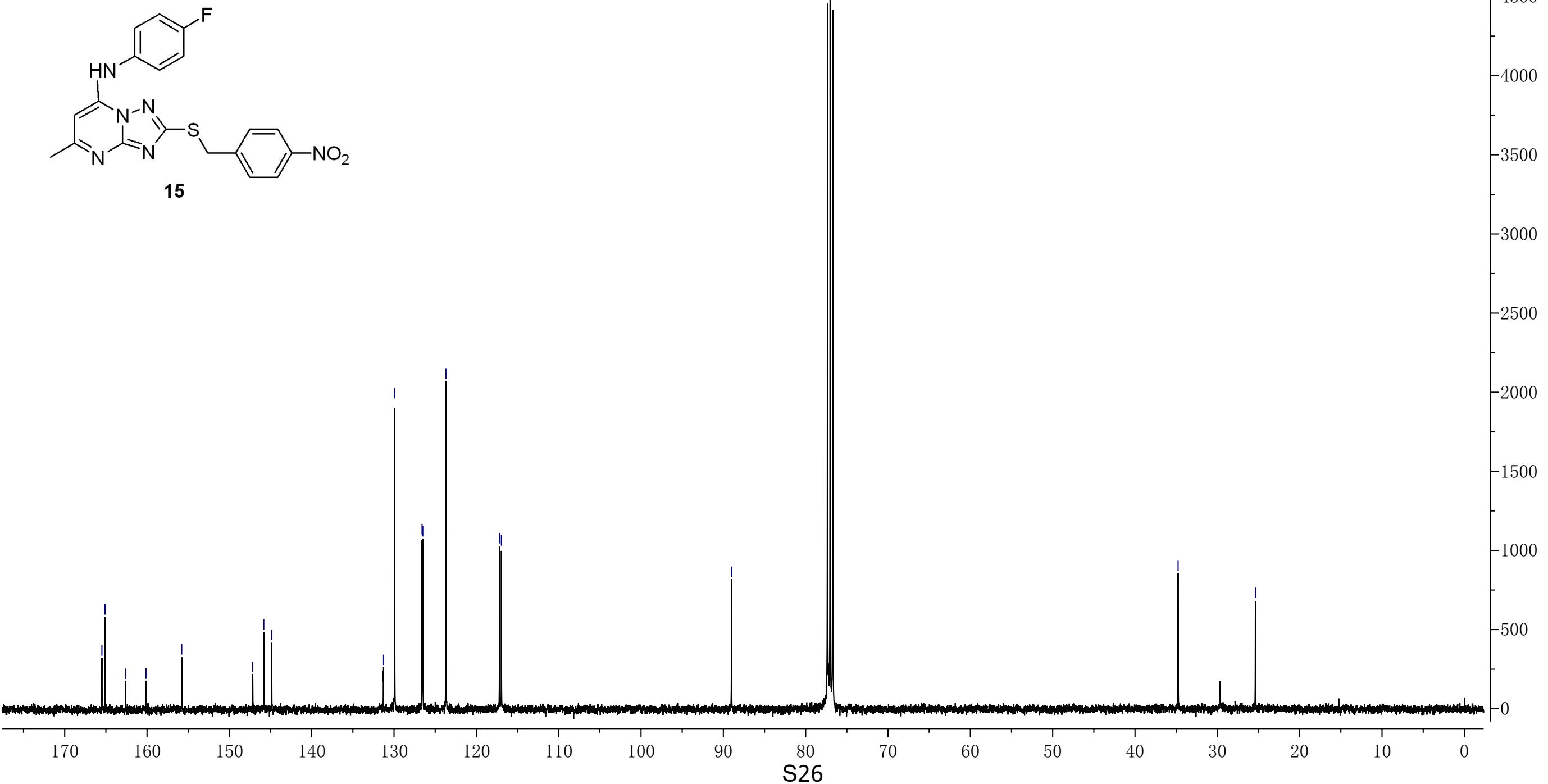

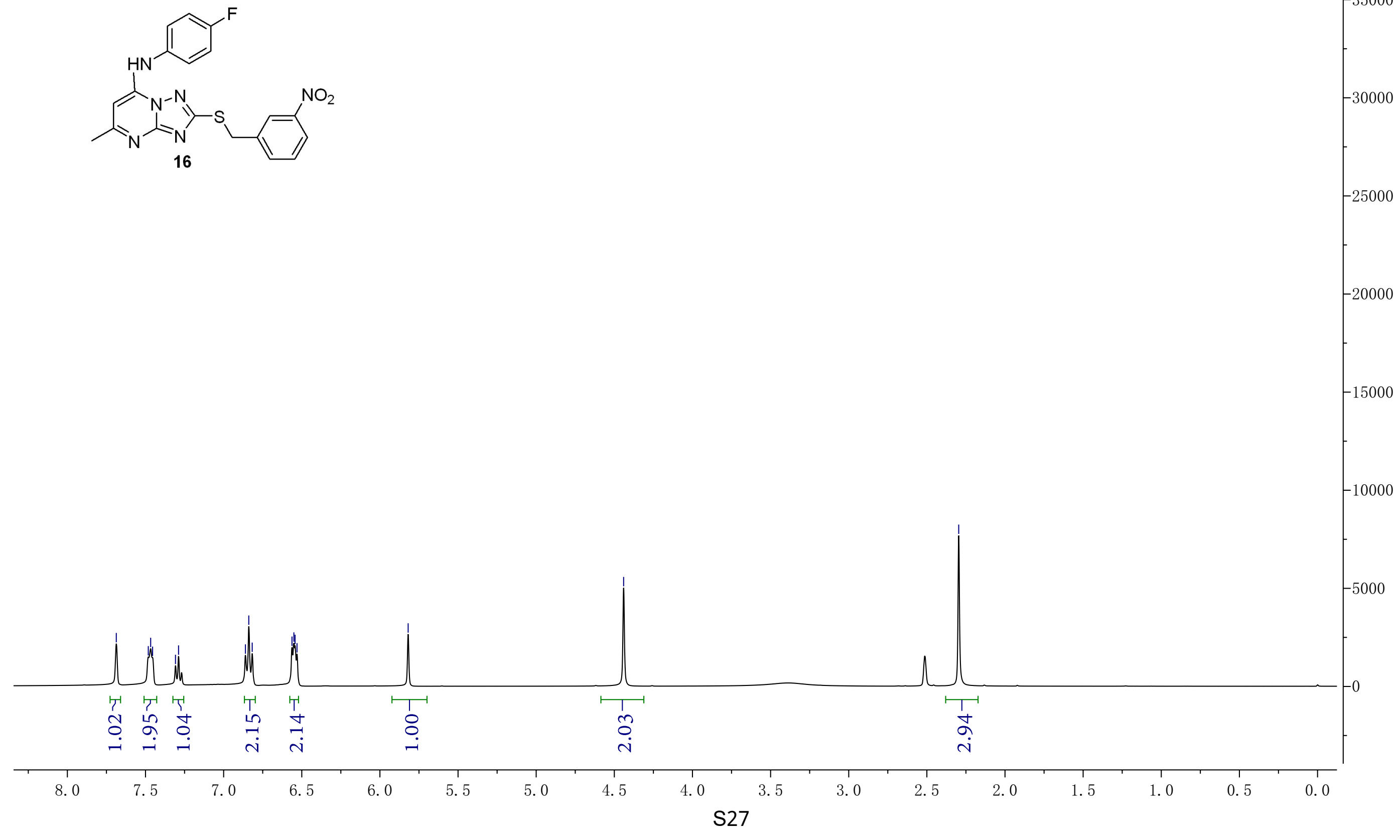

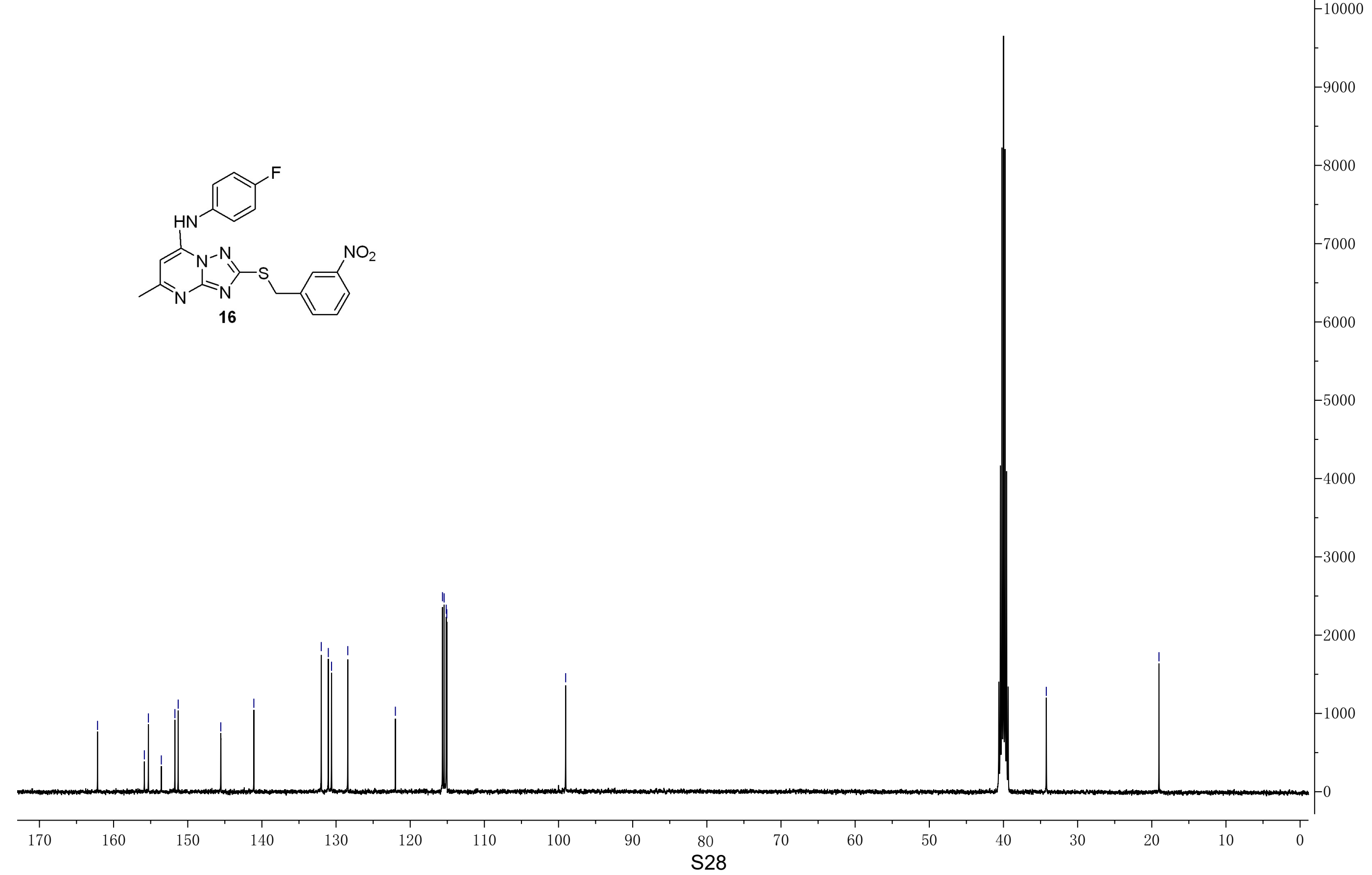

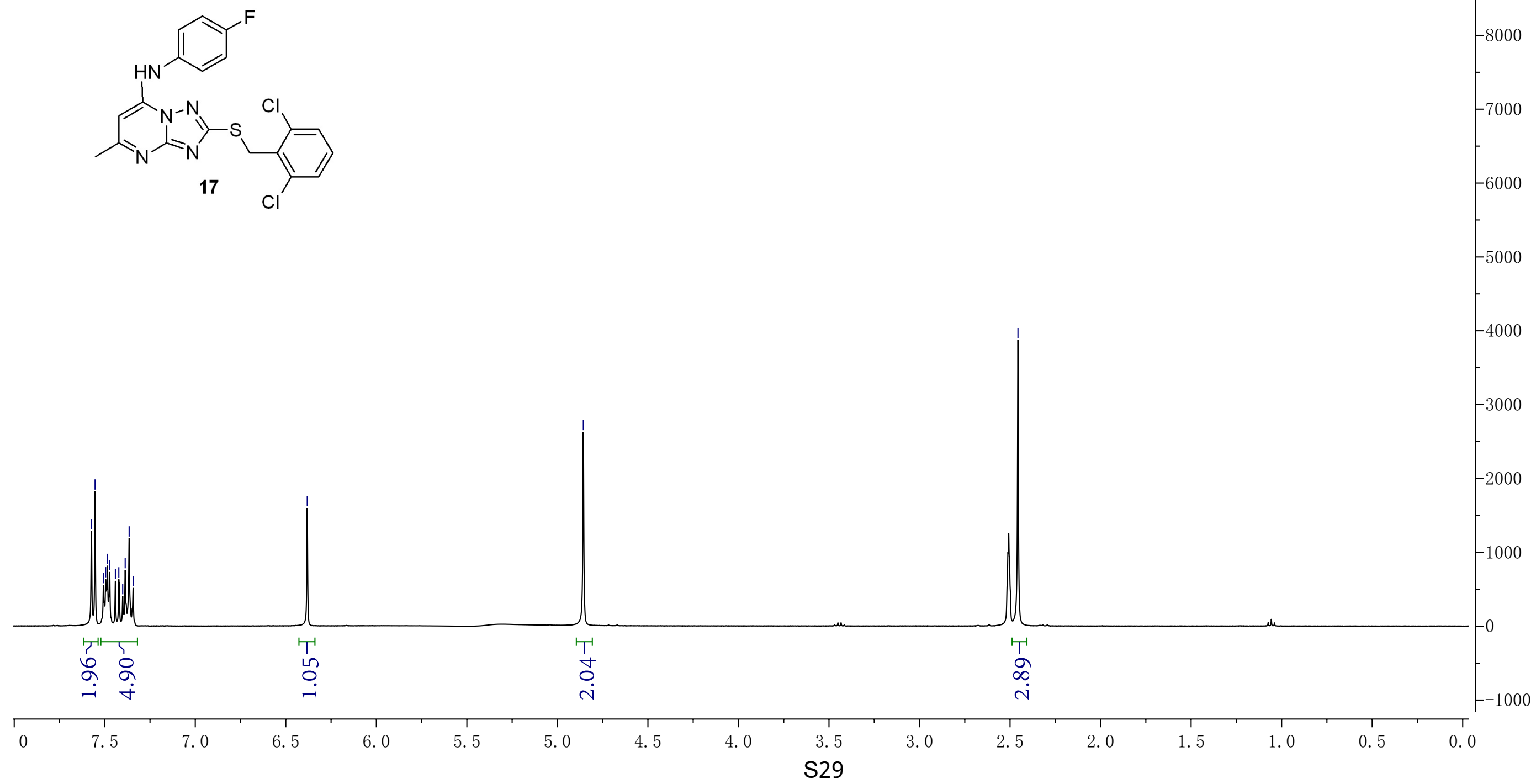


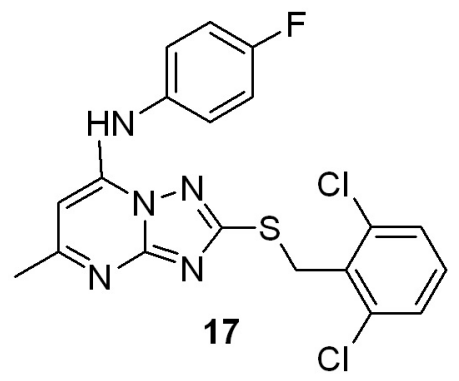

$\begin{array}{ll}\infty & \text { bे } \\ \stackrel{i}{i} & \text { iे }\end{array}$ 

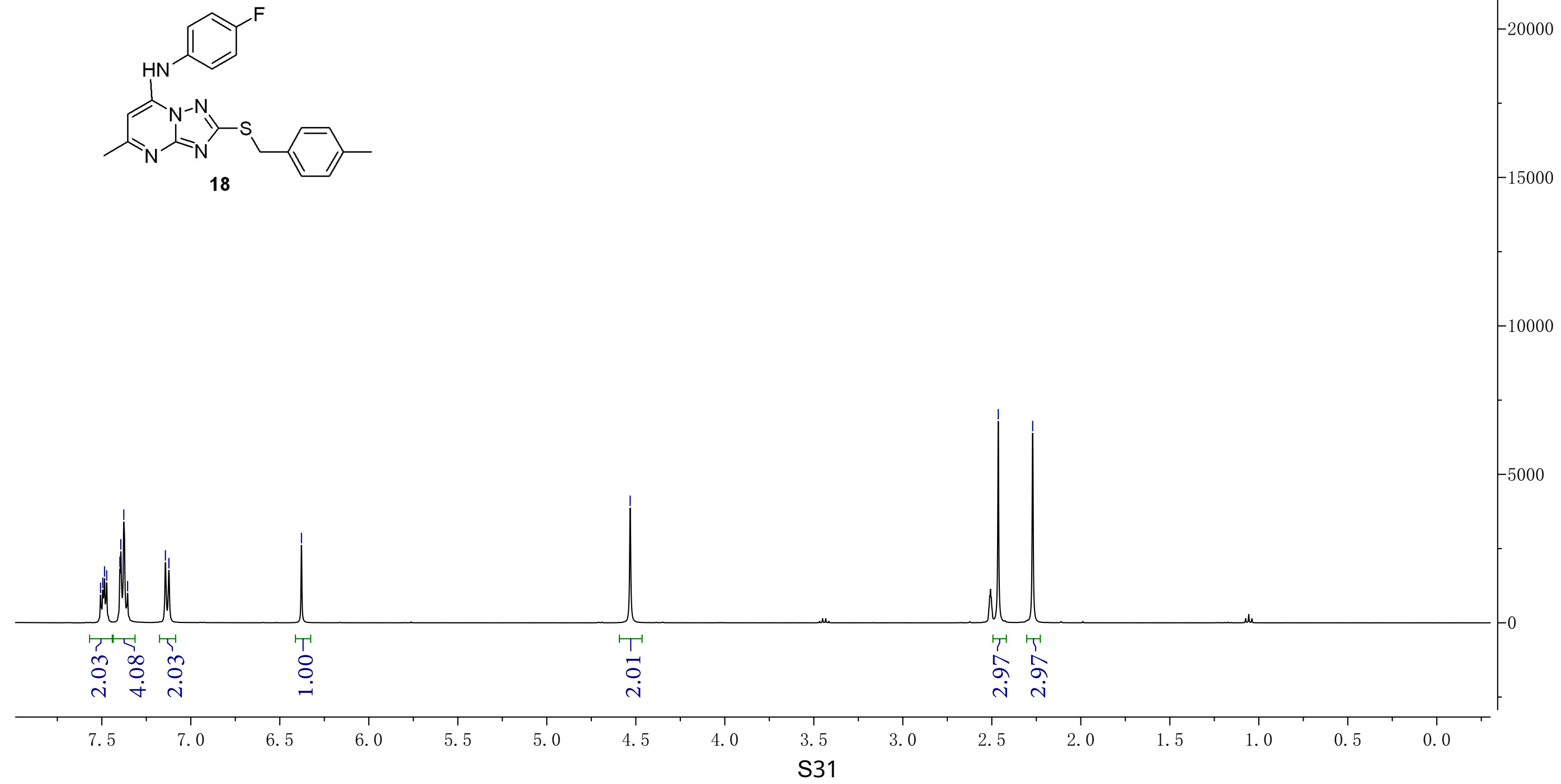

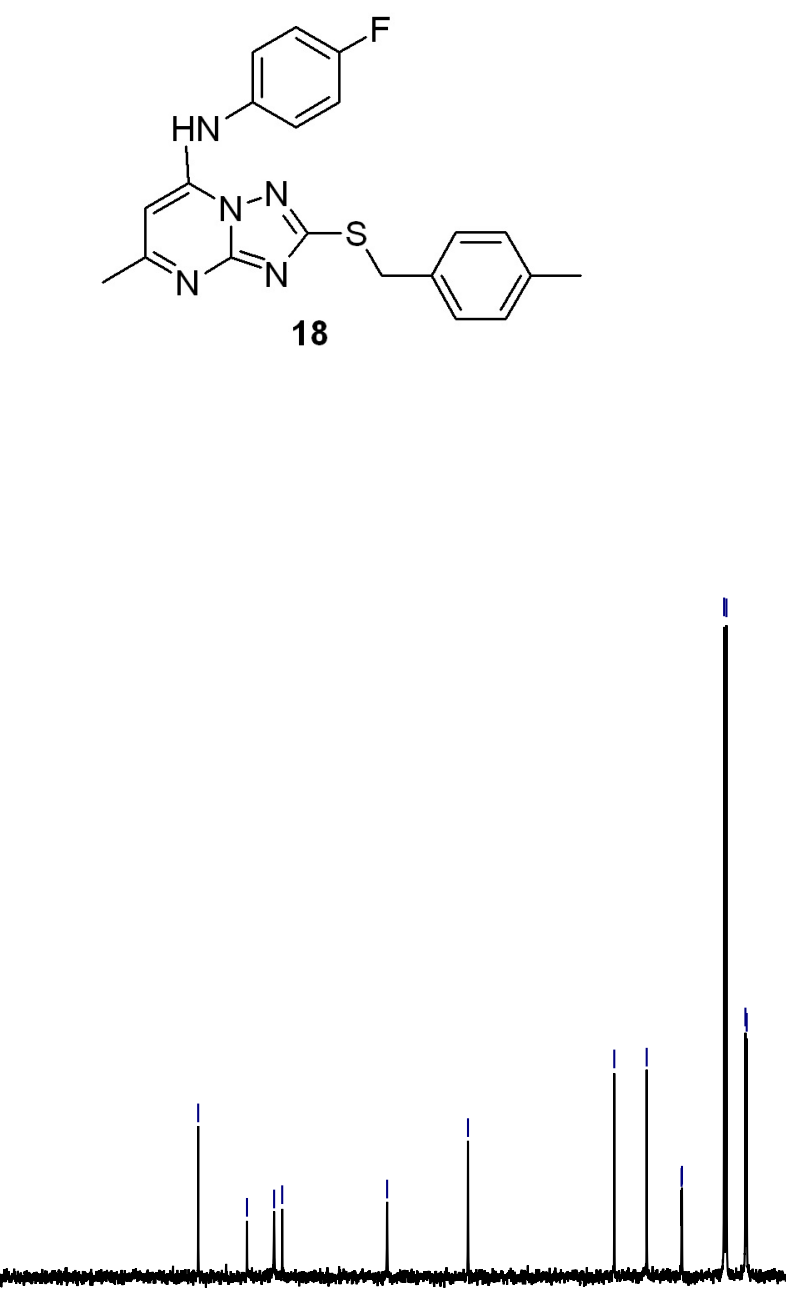

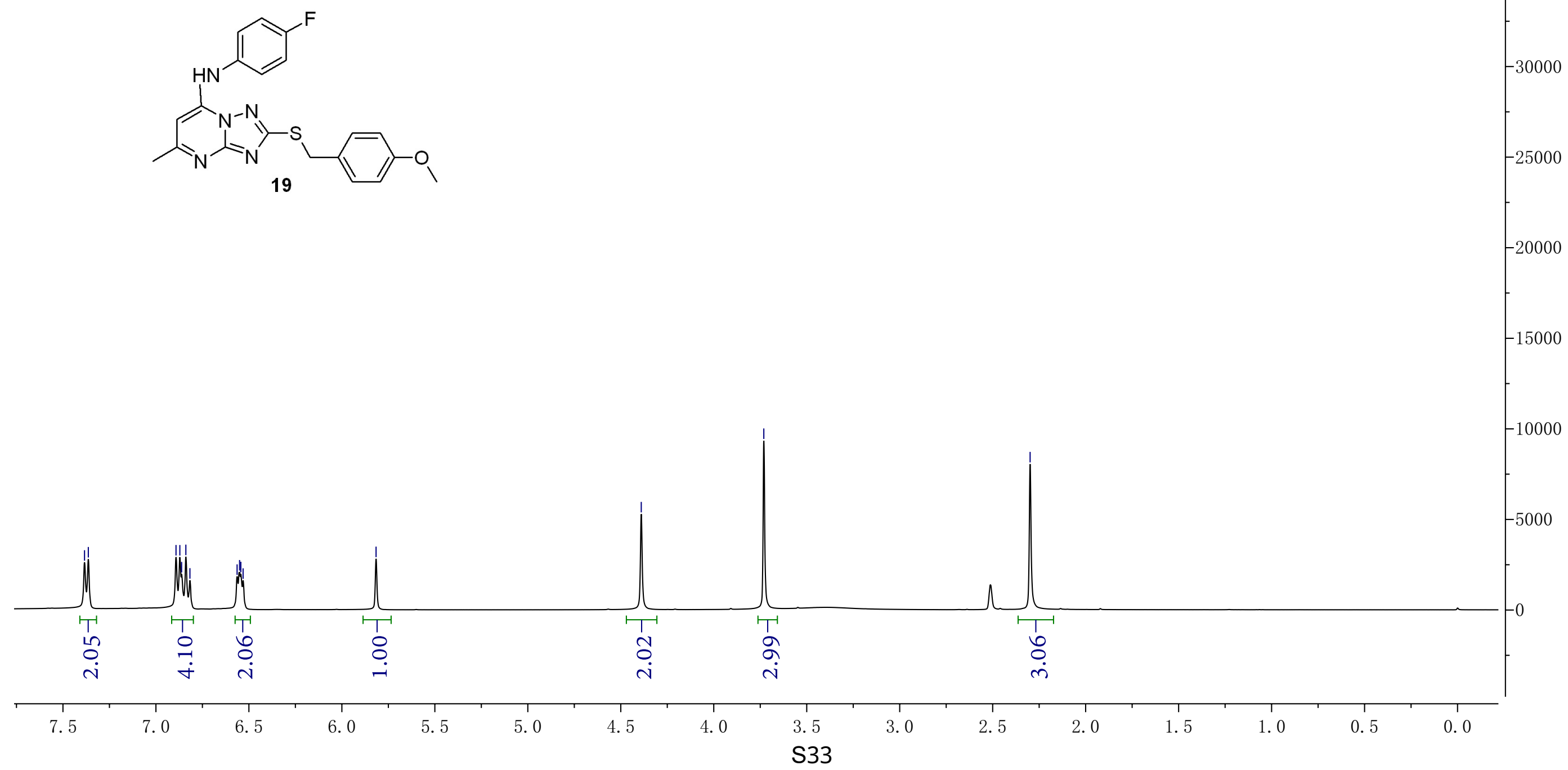

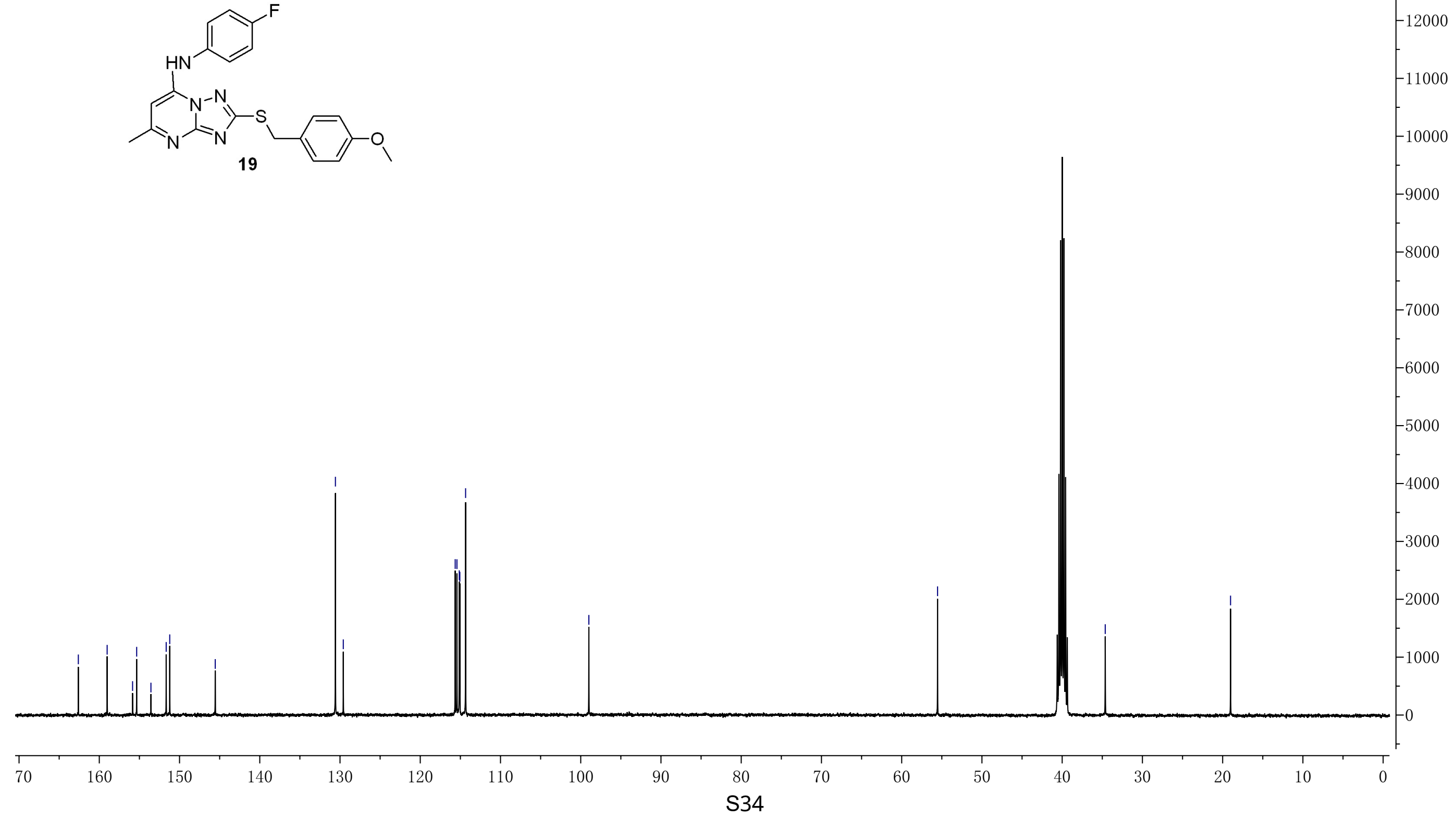

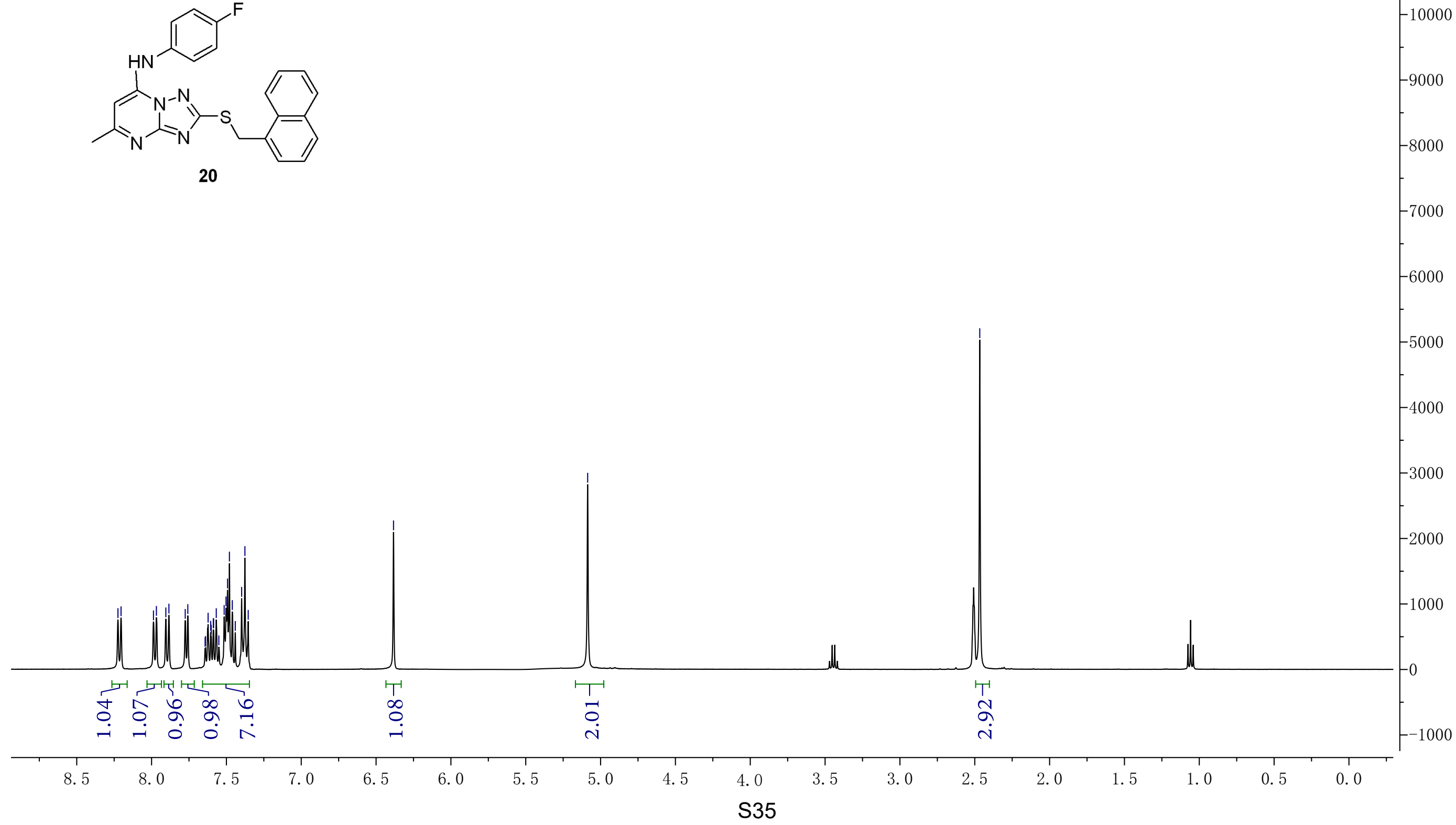

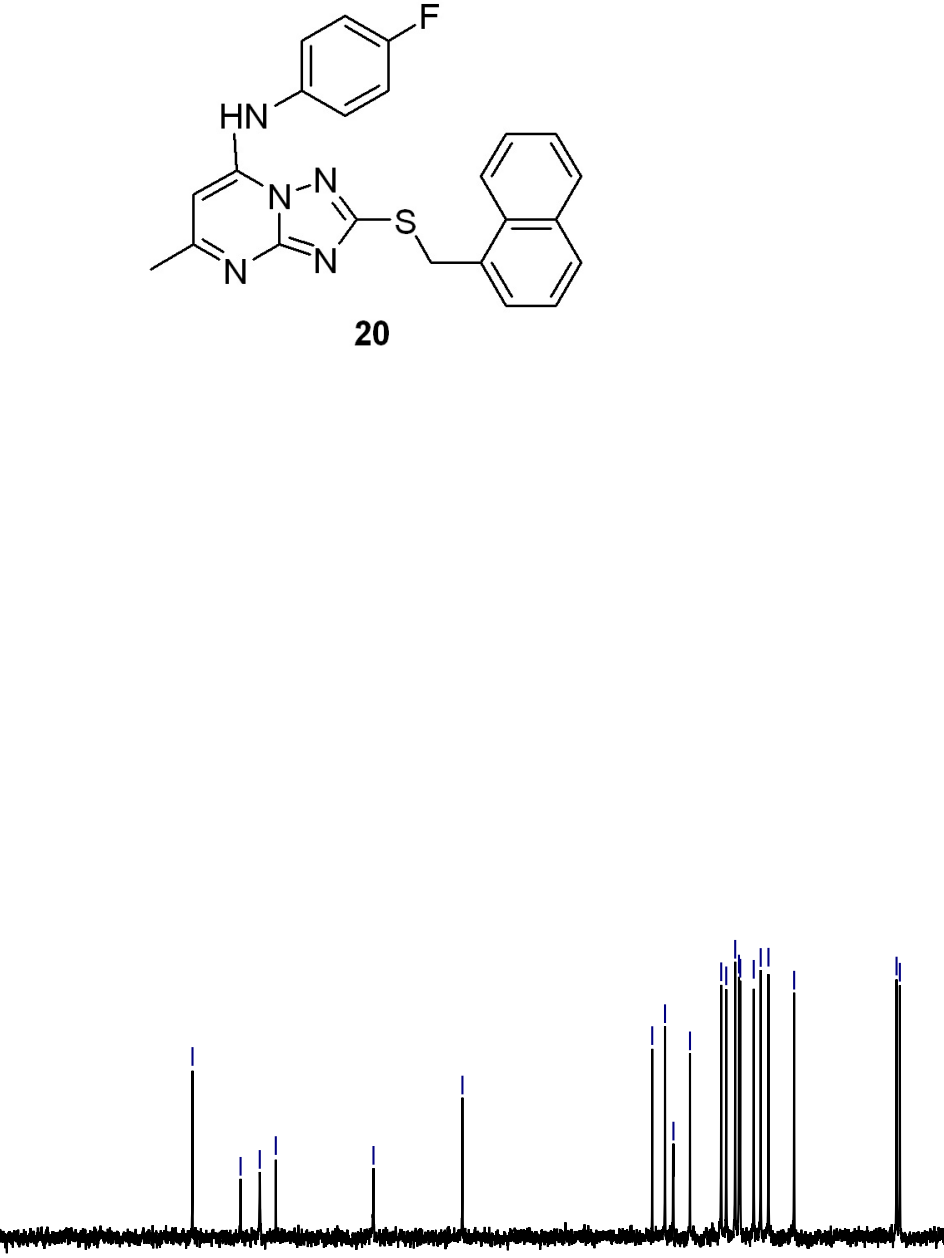

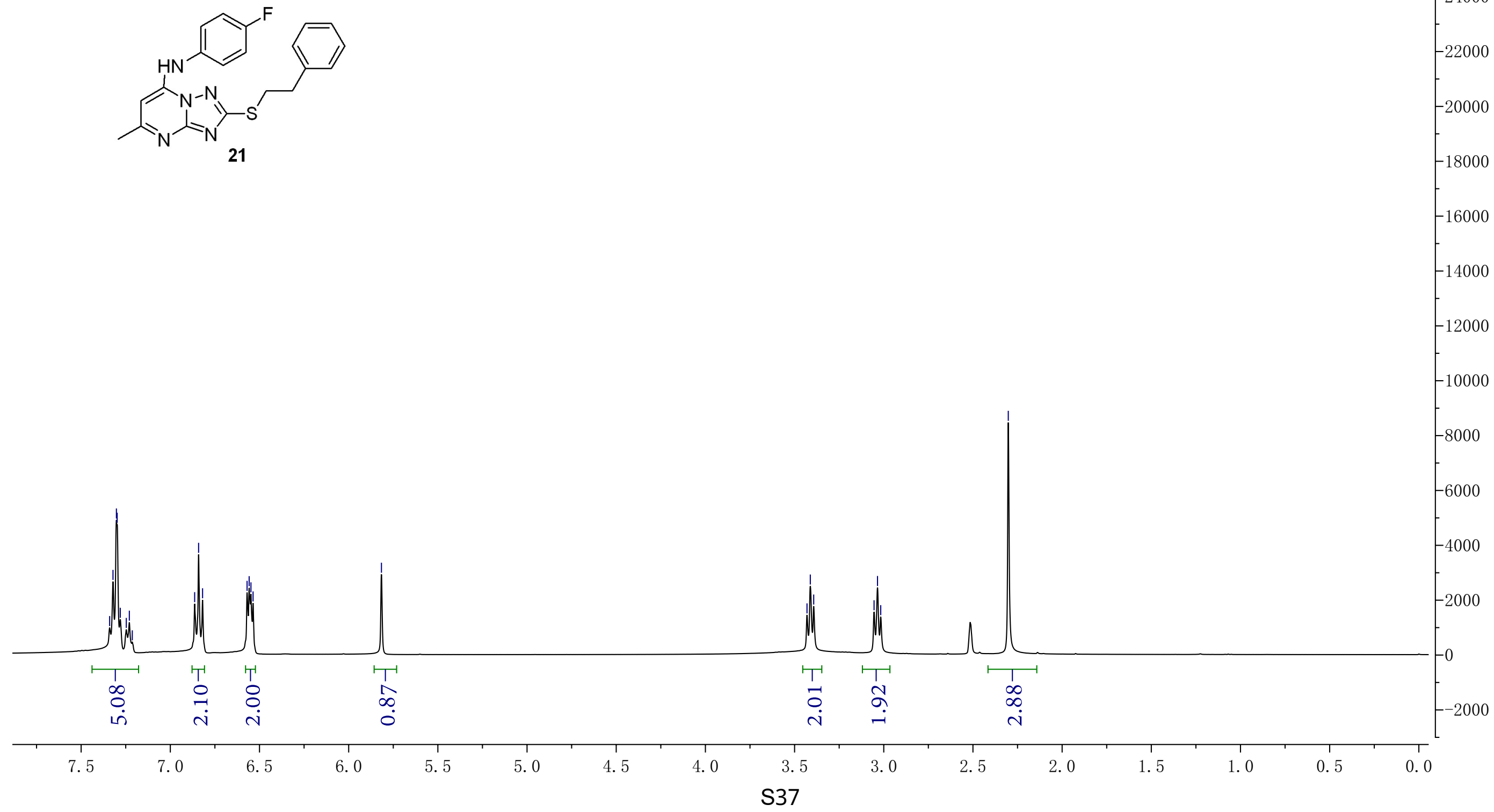

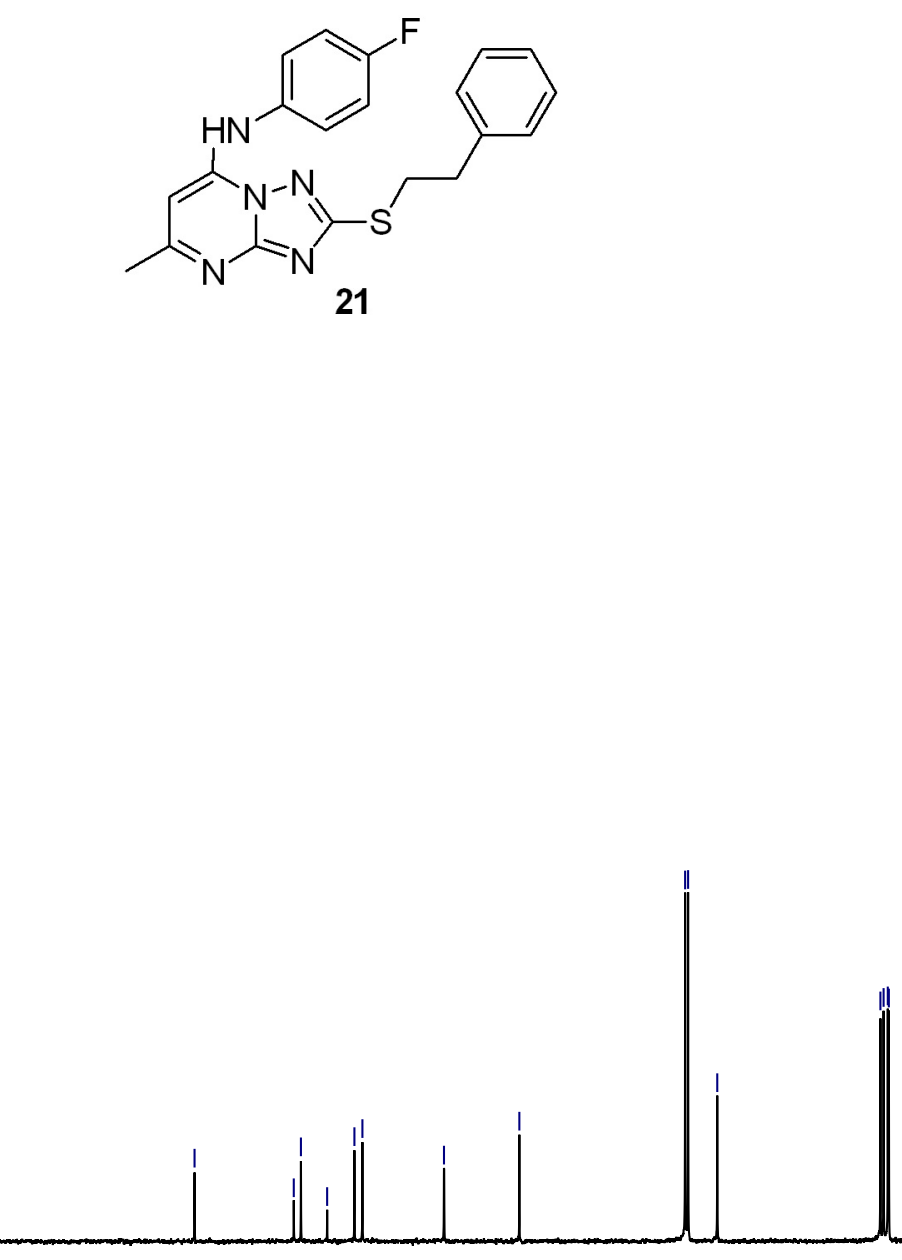

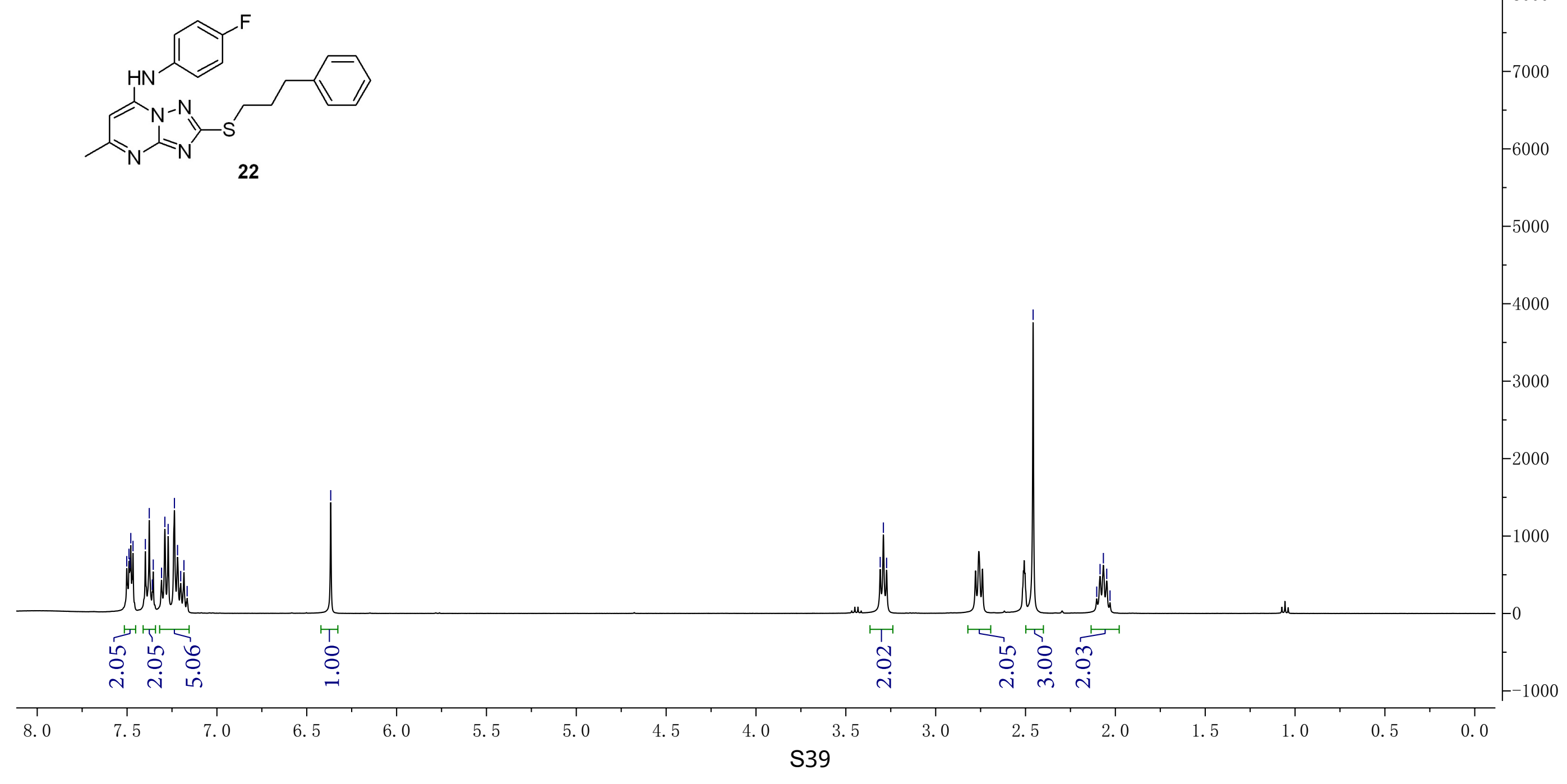

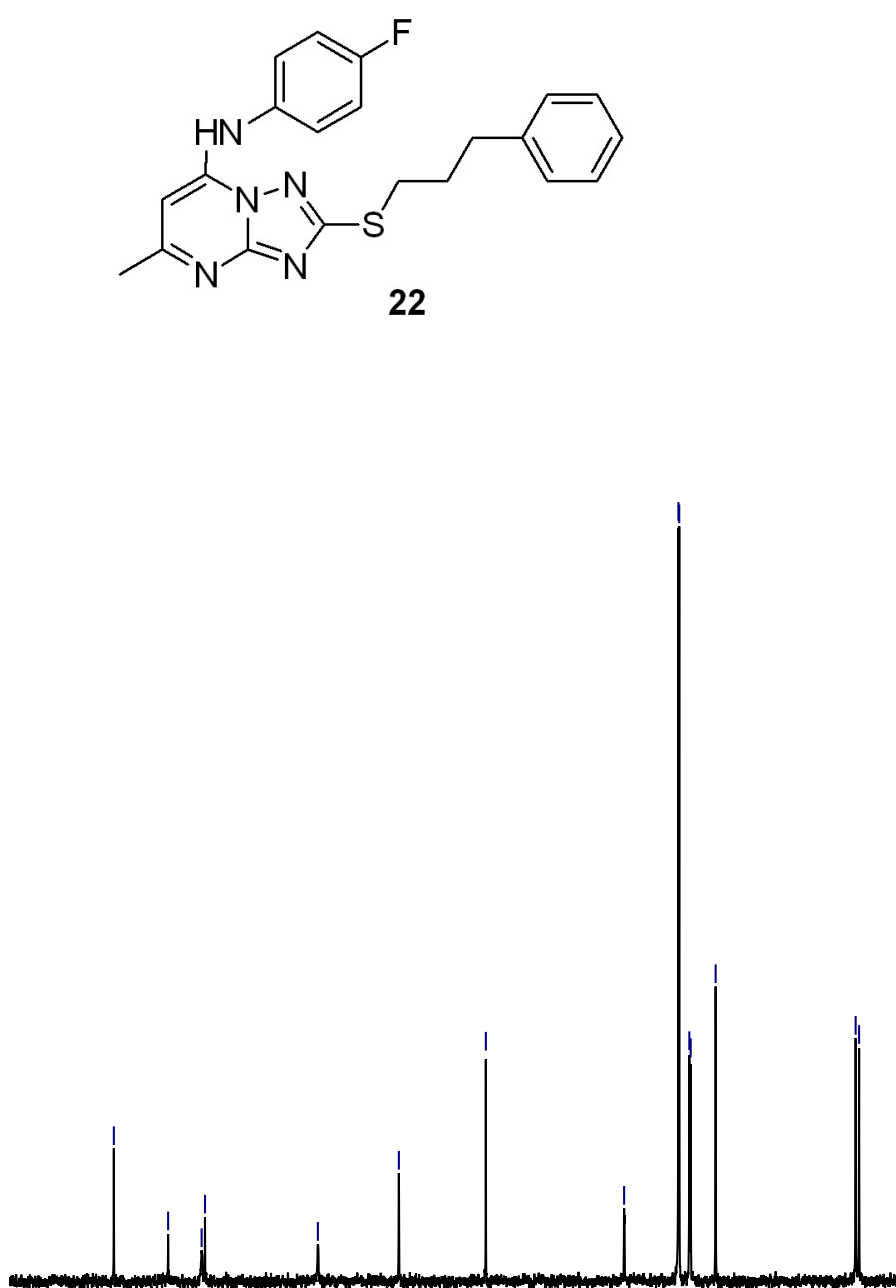

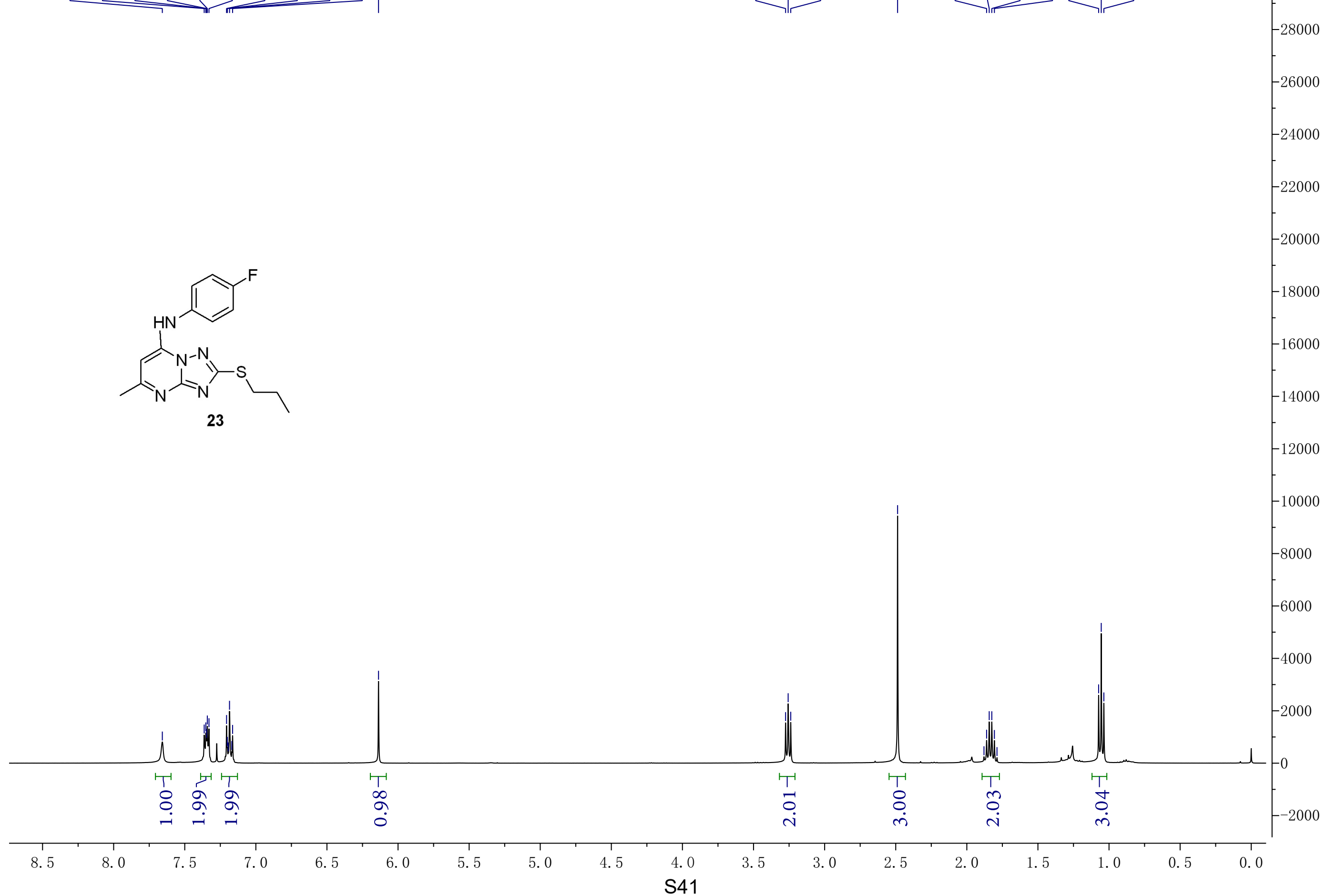


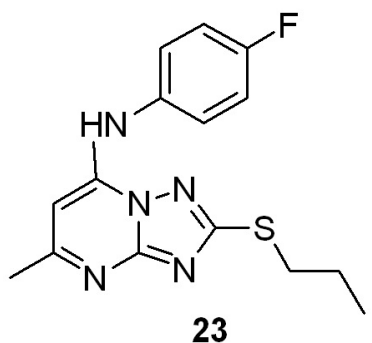



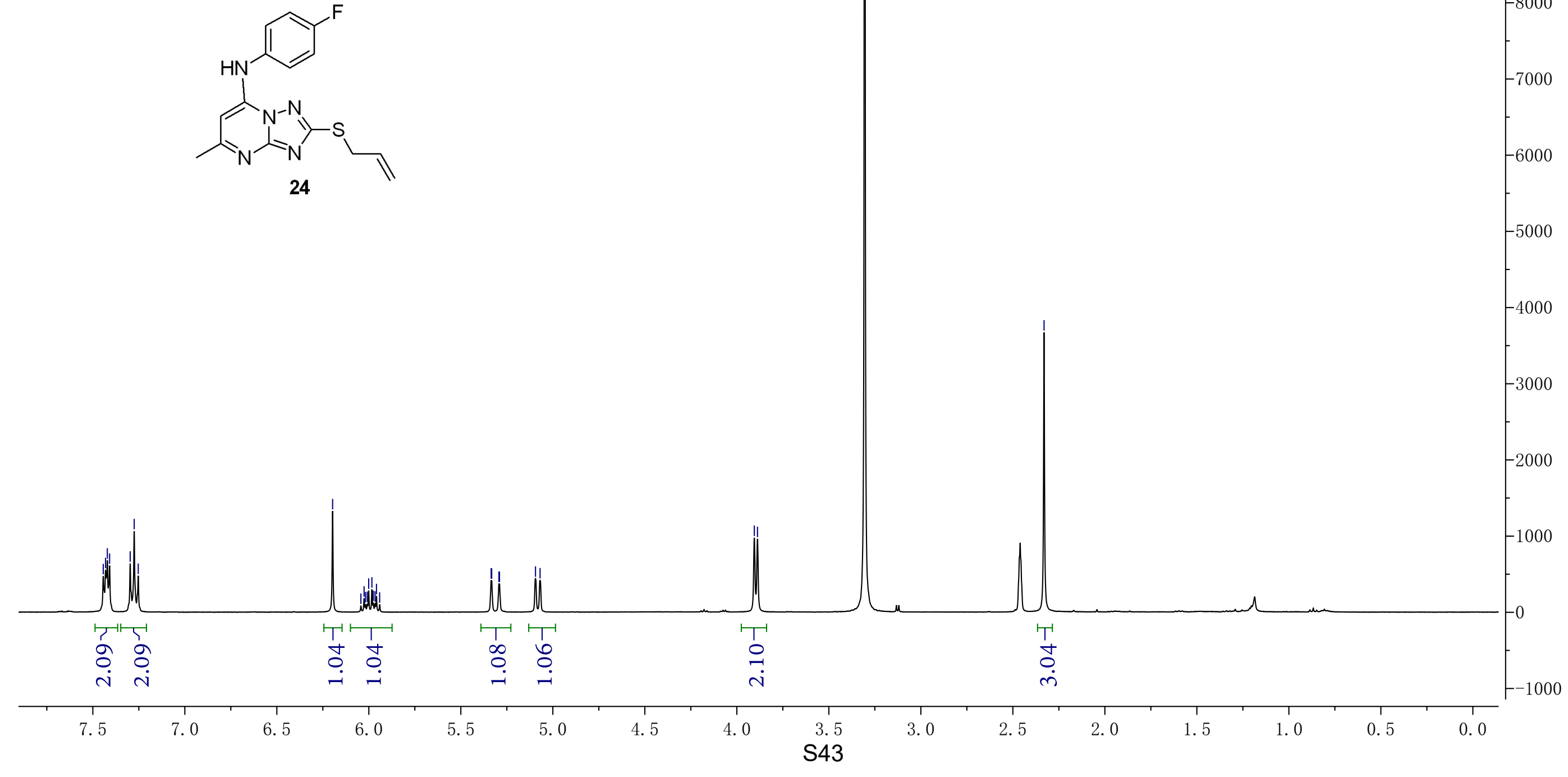


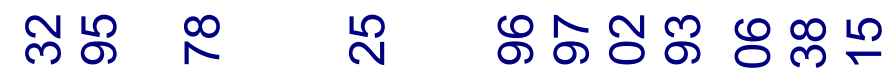

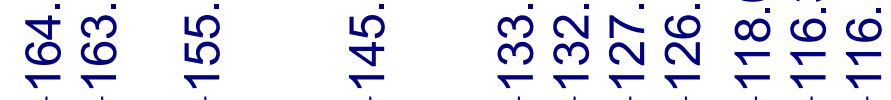

$\stackrel{\sim}{\infty}$

กิ
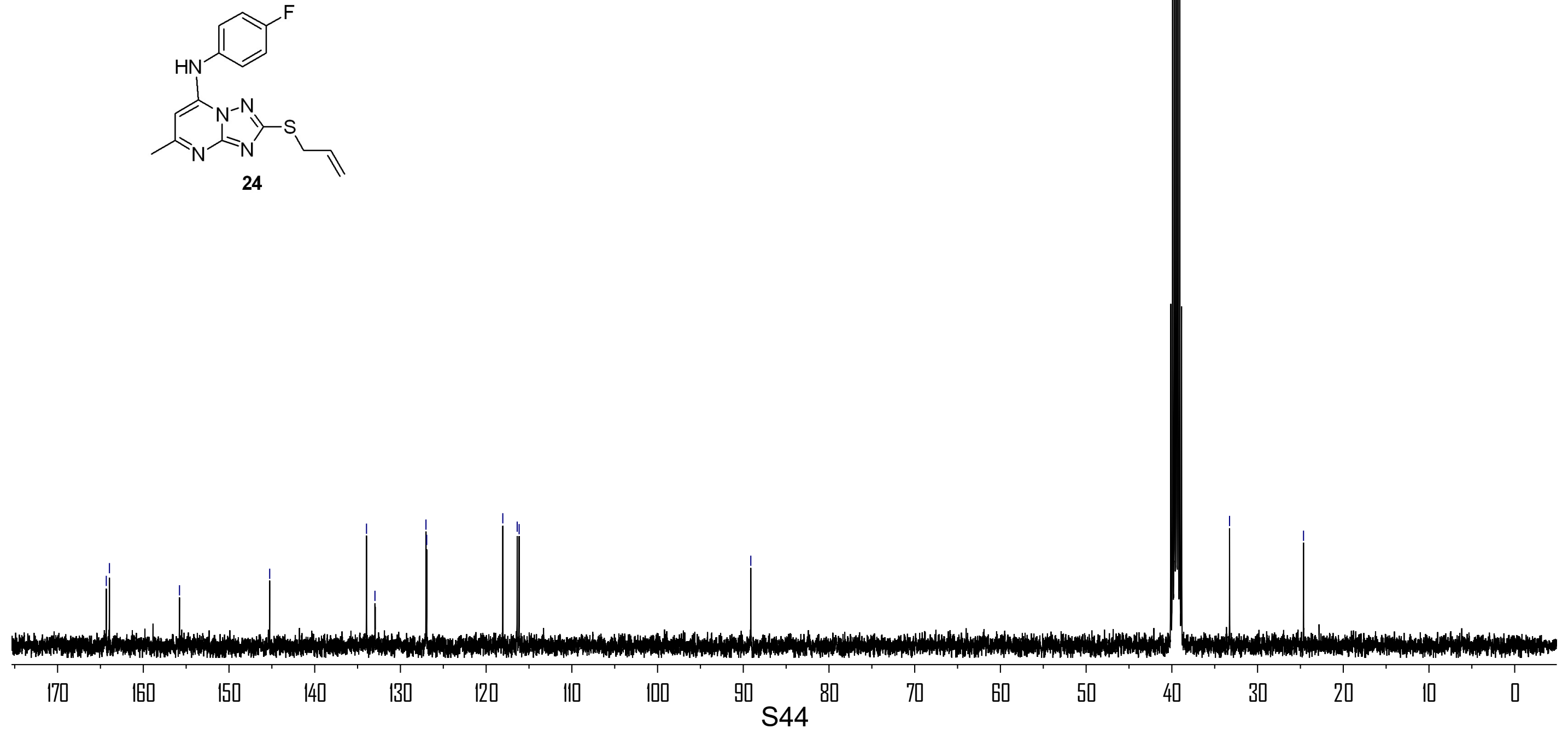

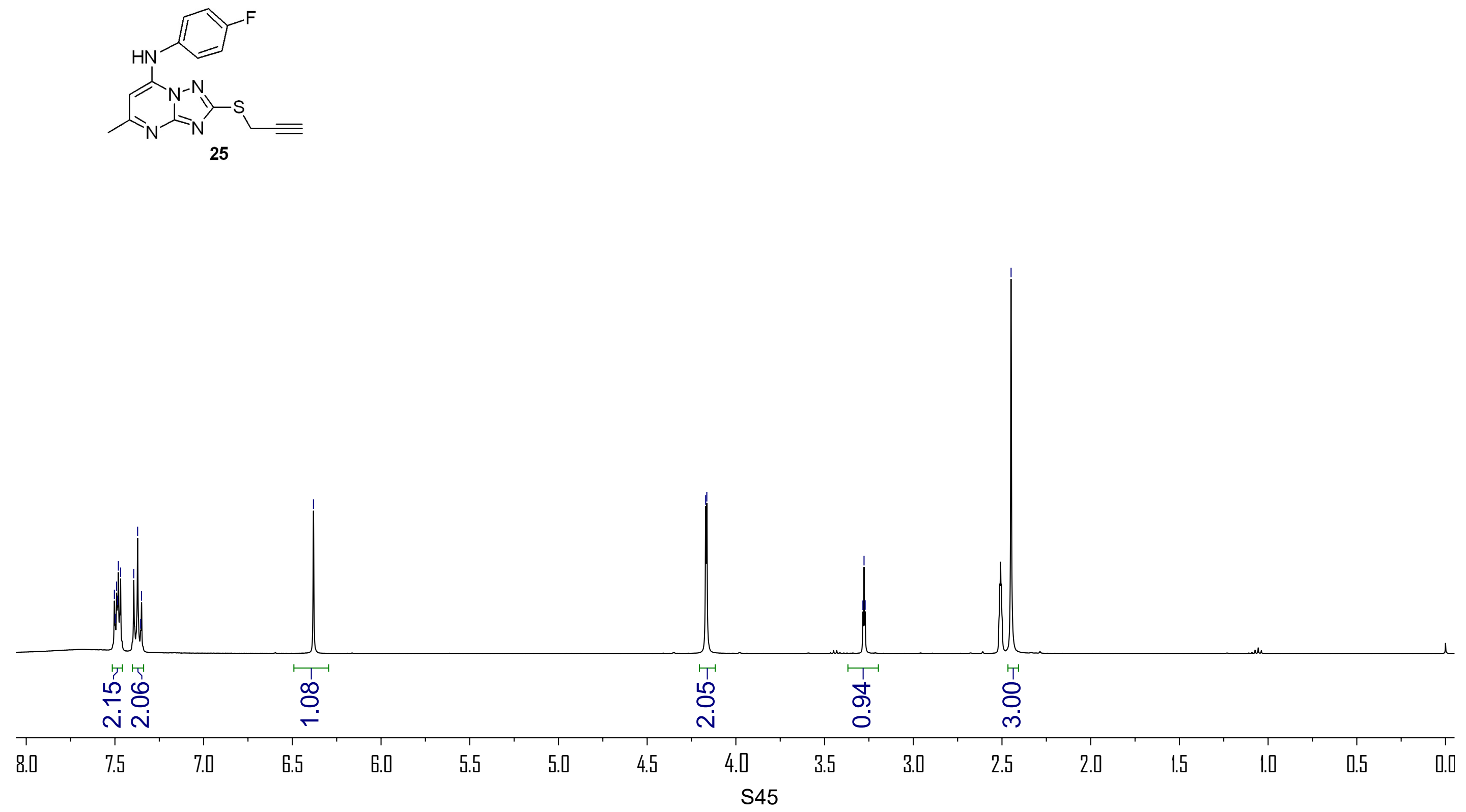
니ㅇㅛㅠ 용

ษั

ํ

ชิ ซ

ิㅗํ ํㅡㄷ

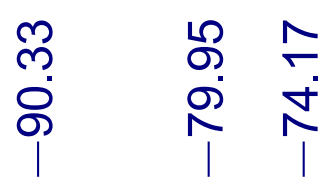

nิ

ํํํ
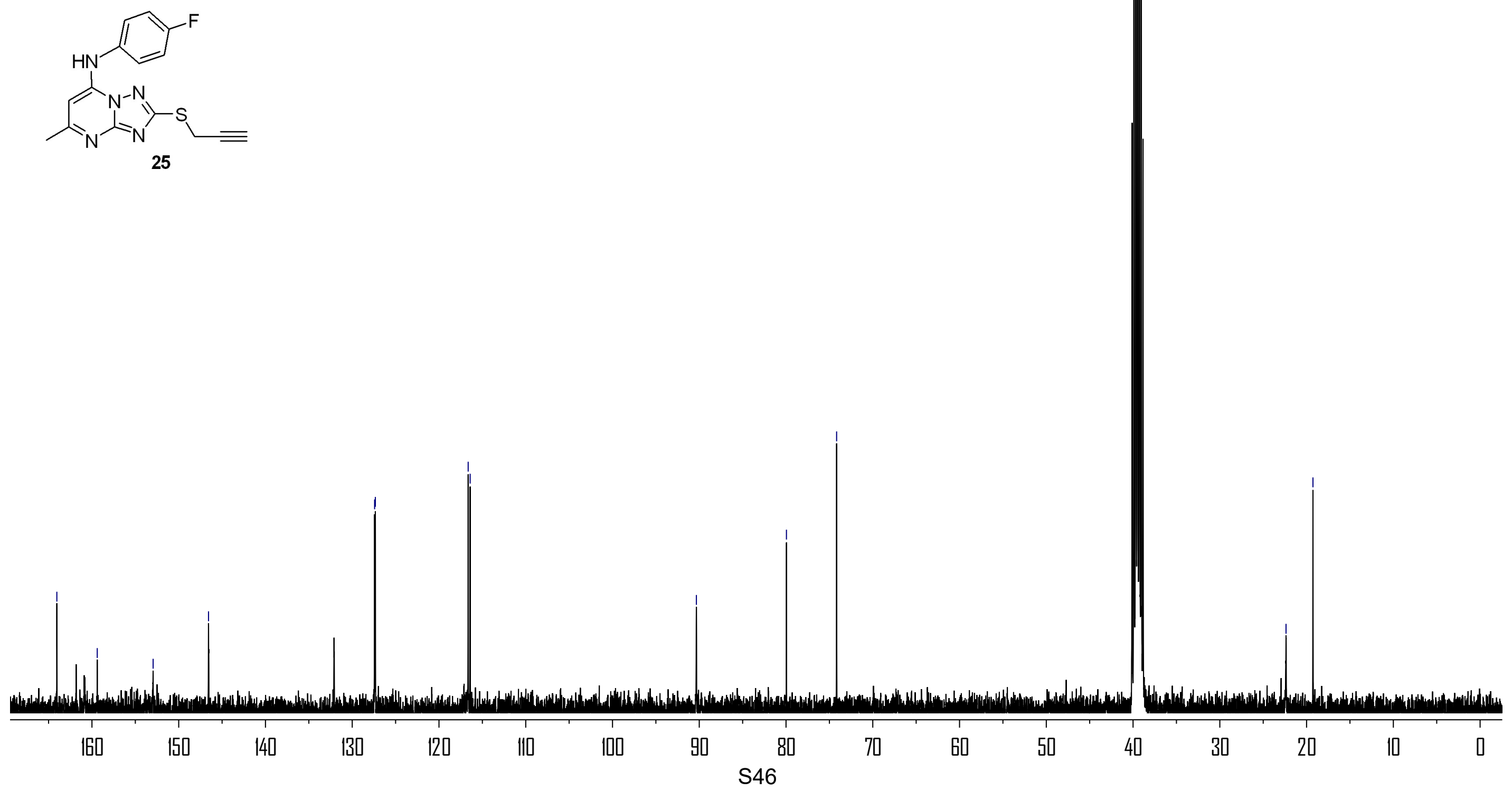

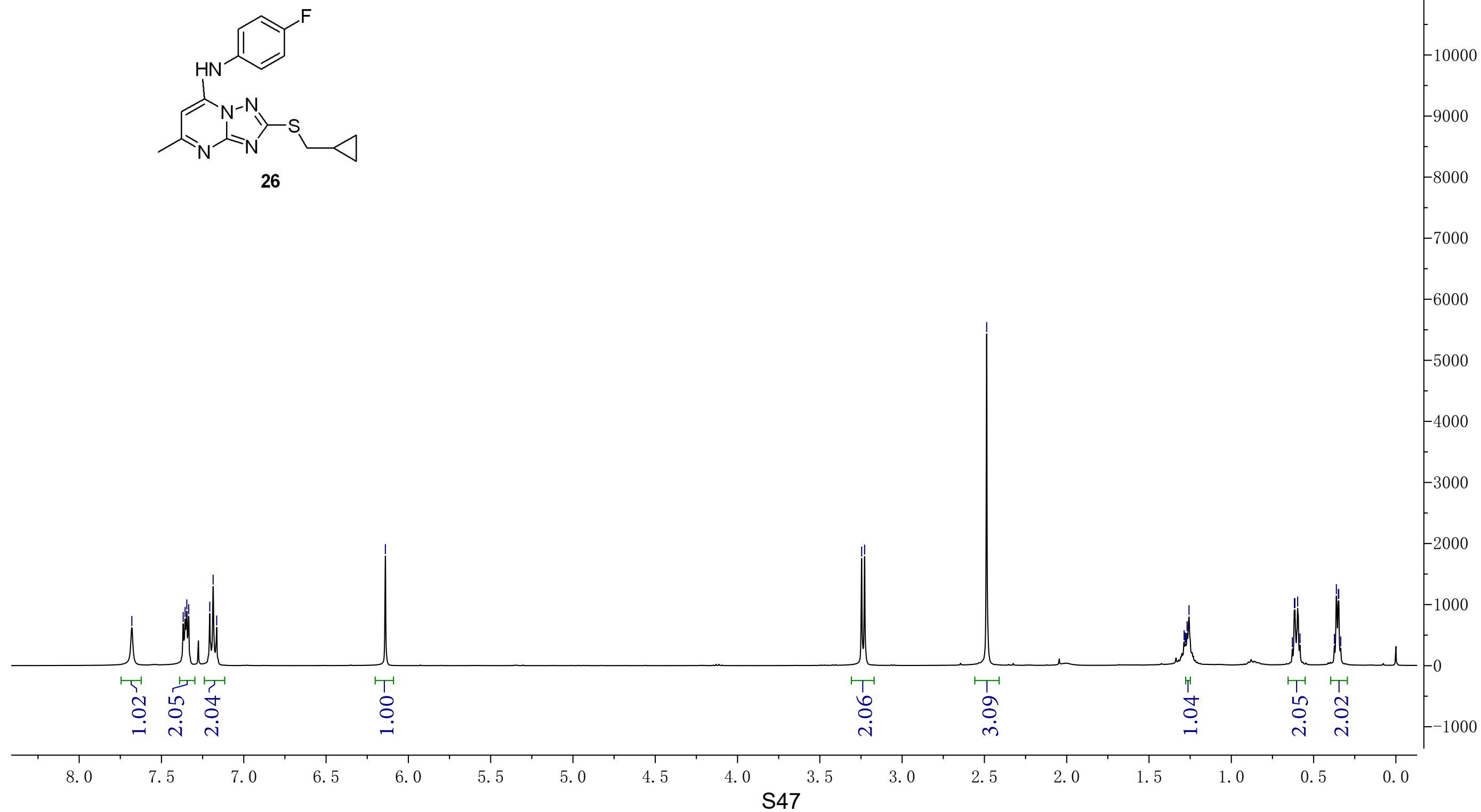
tras<smiles>Cc1cc(Nc2ccc(F)cc2)n2nc(SCC3CC3)nc2n1</smiles>

26

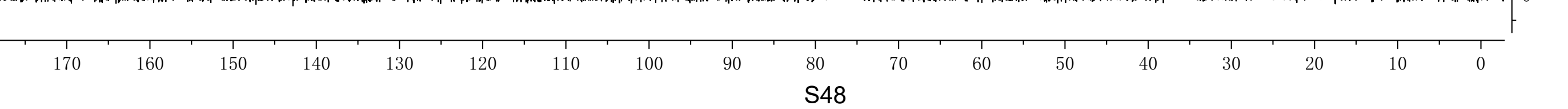



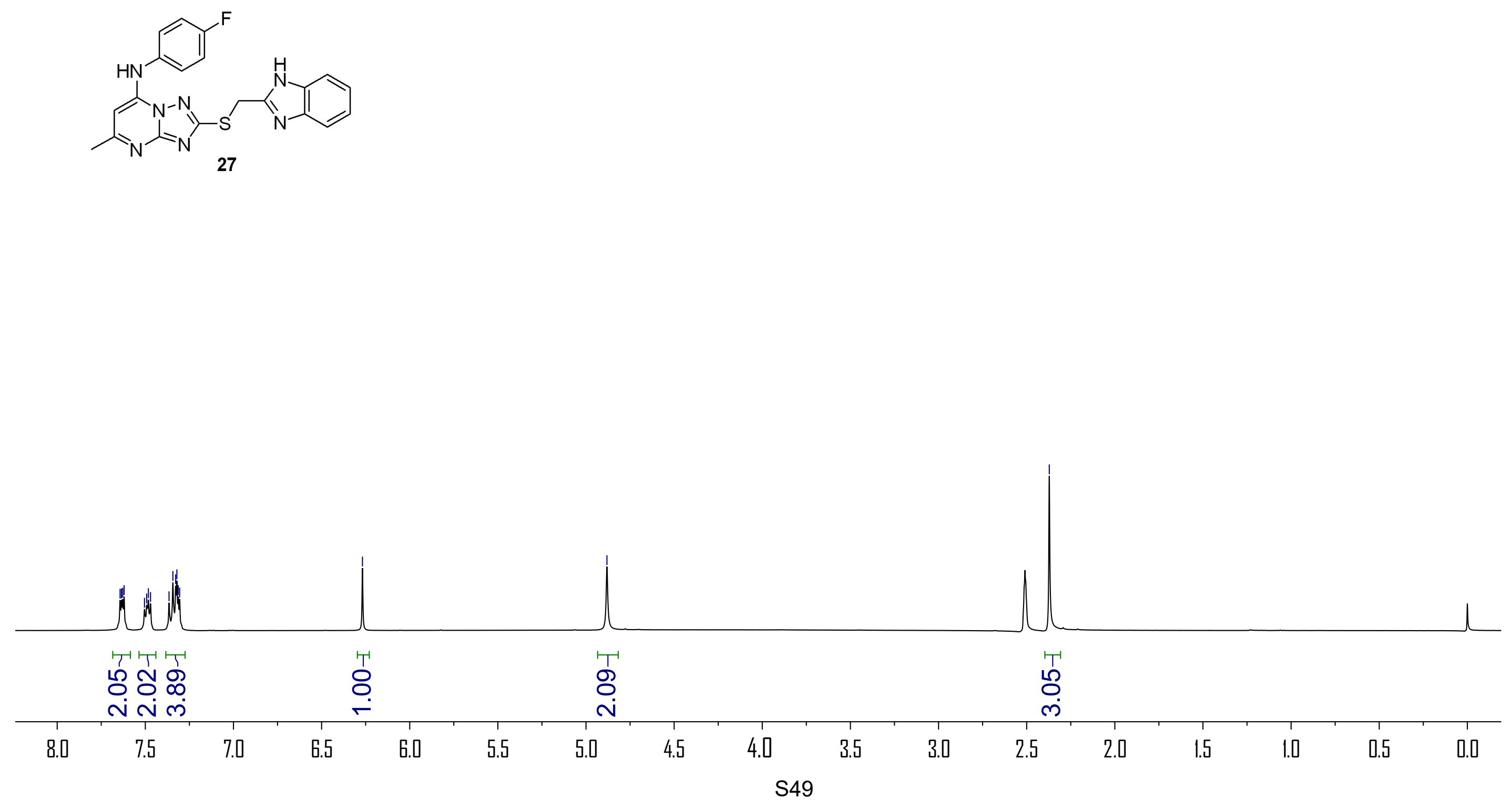

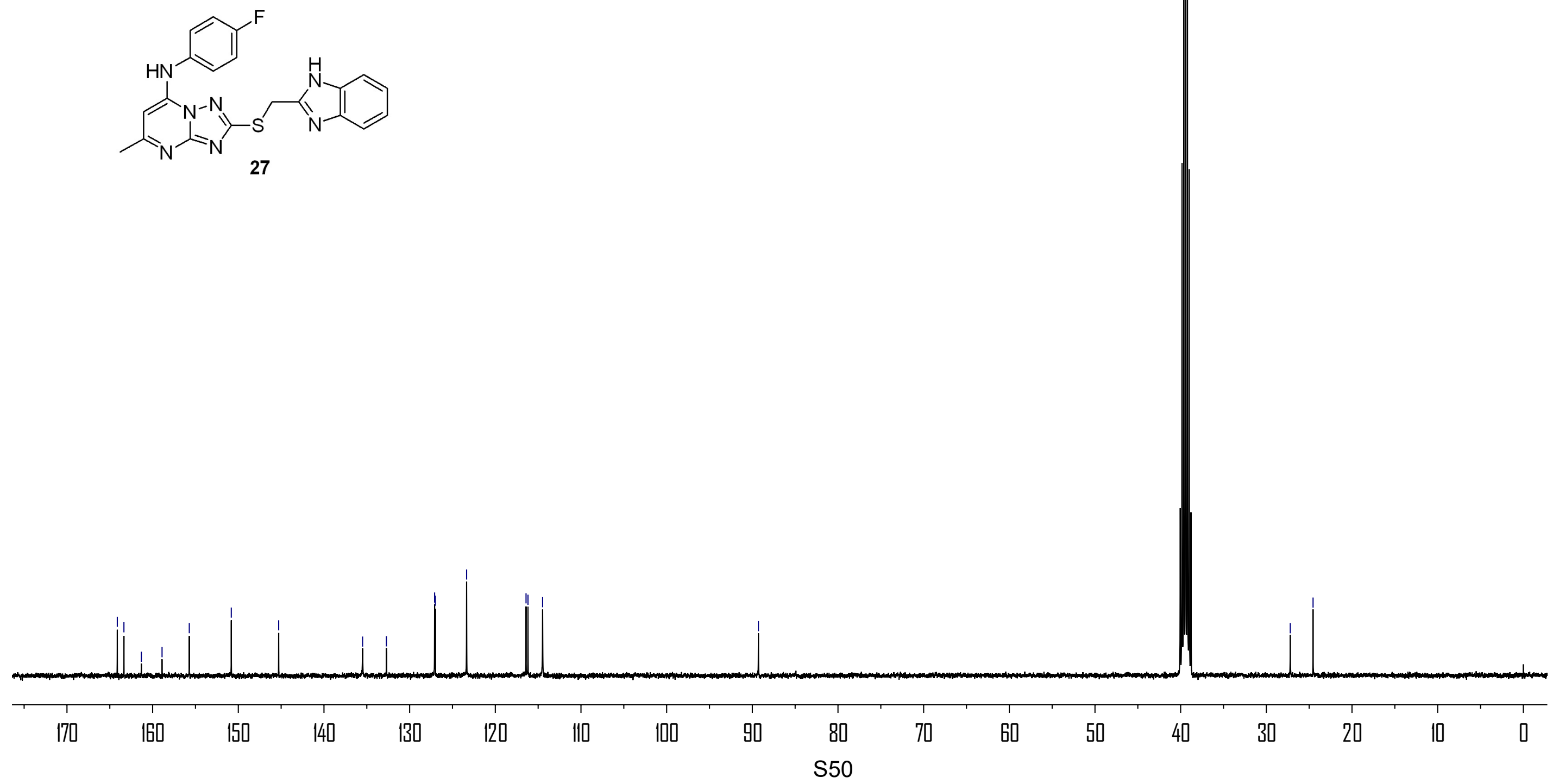

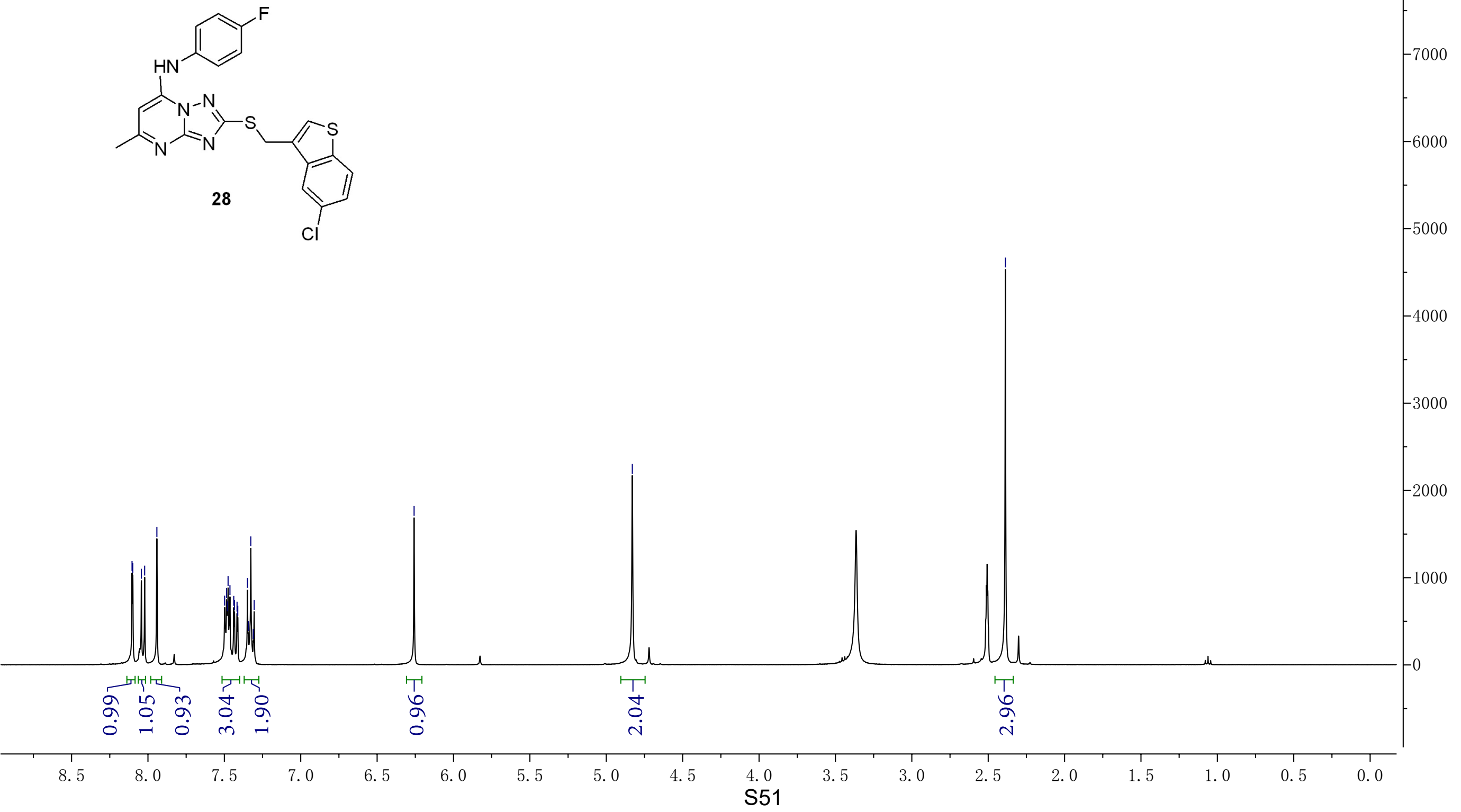

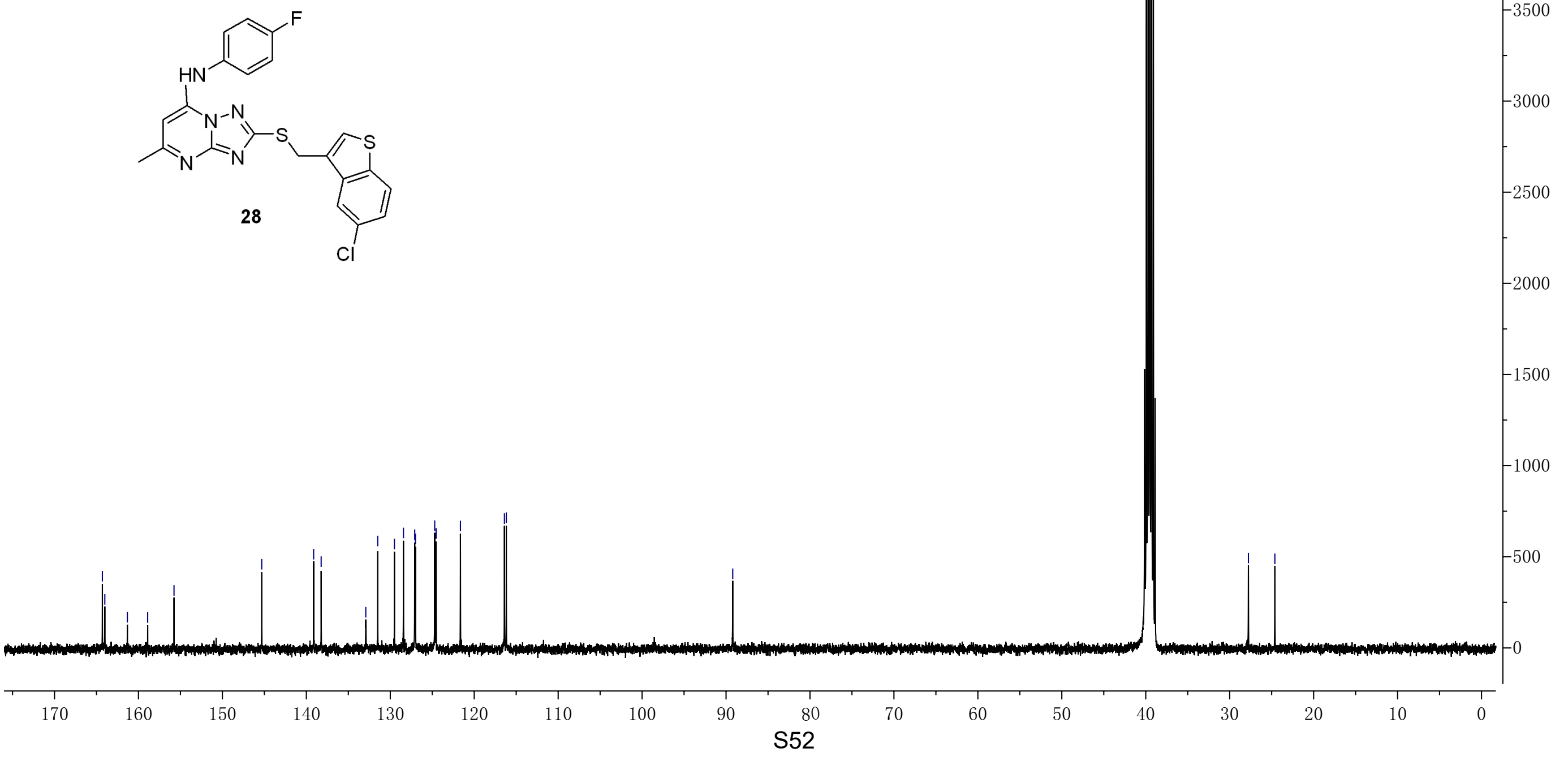

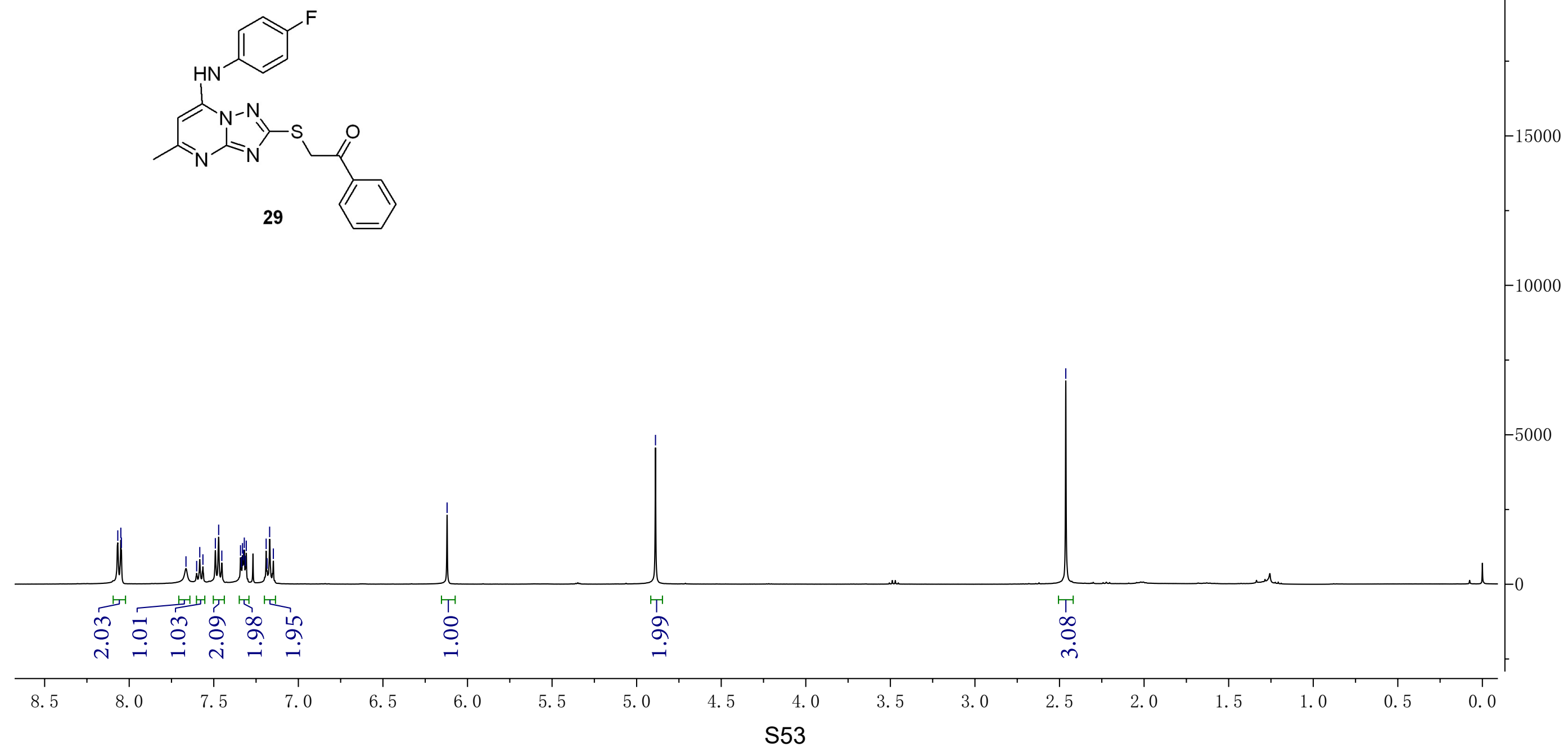


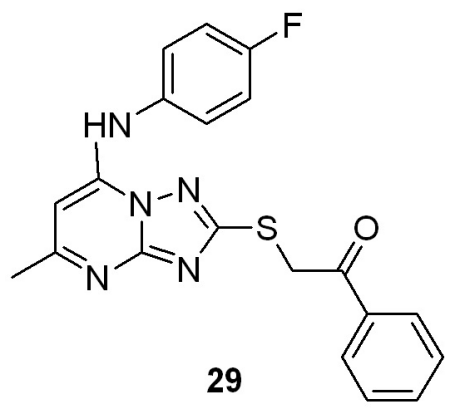




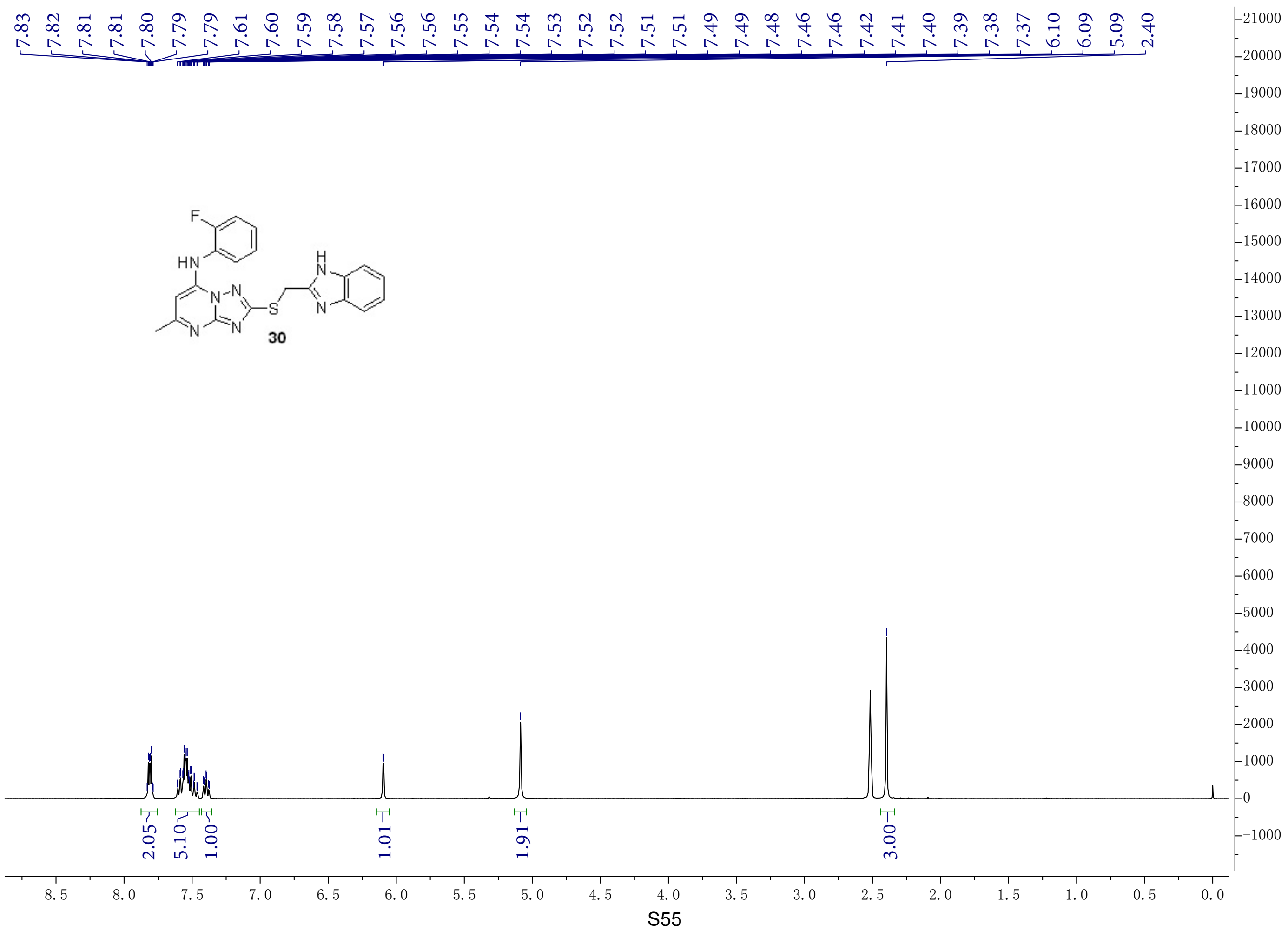



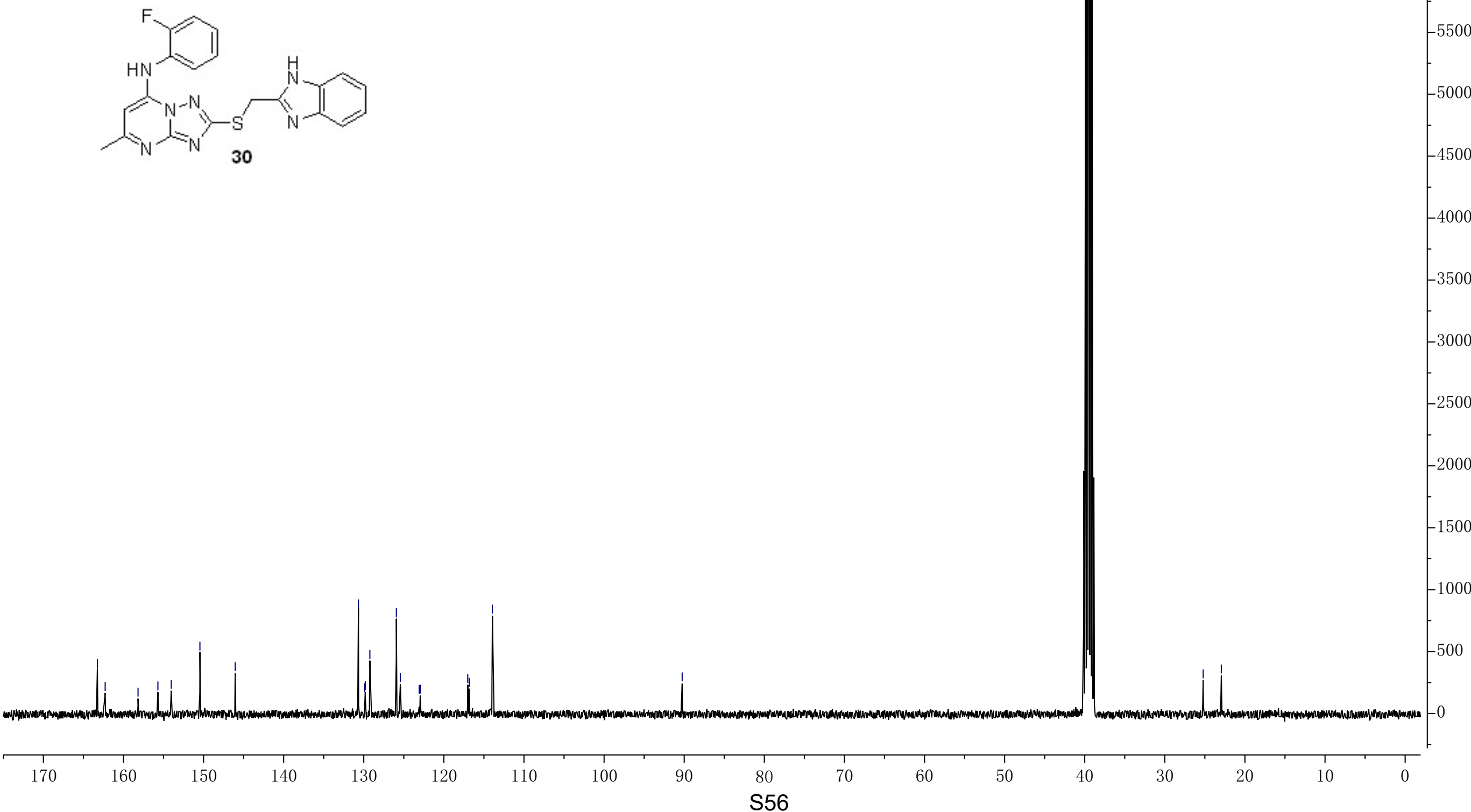

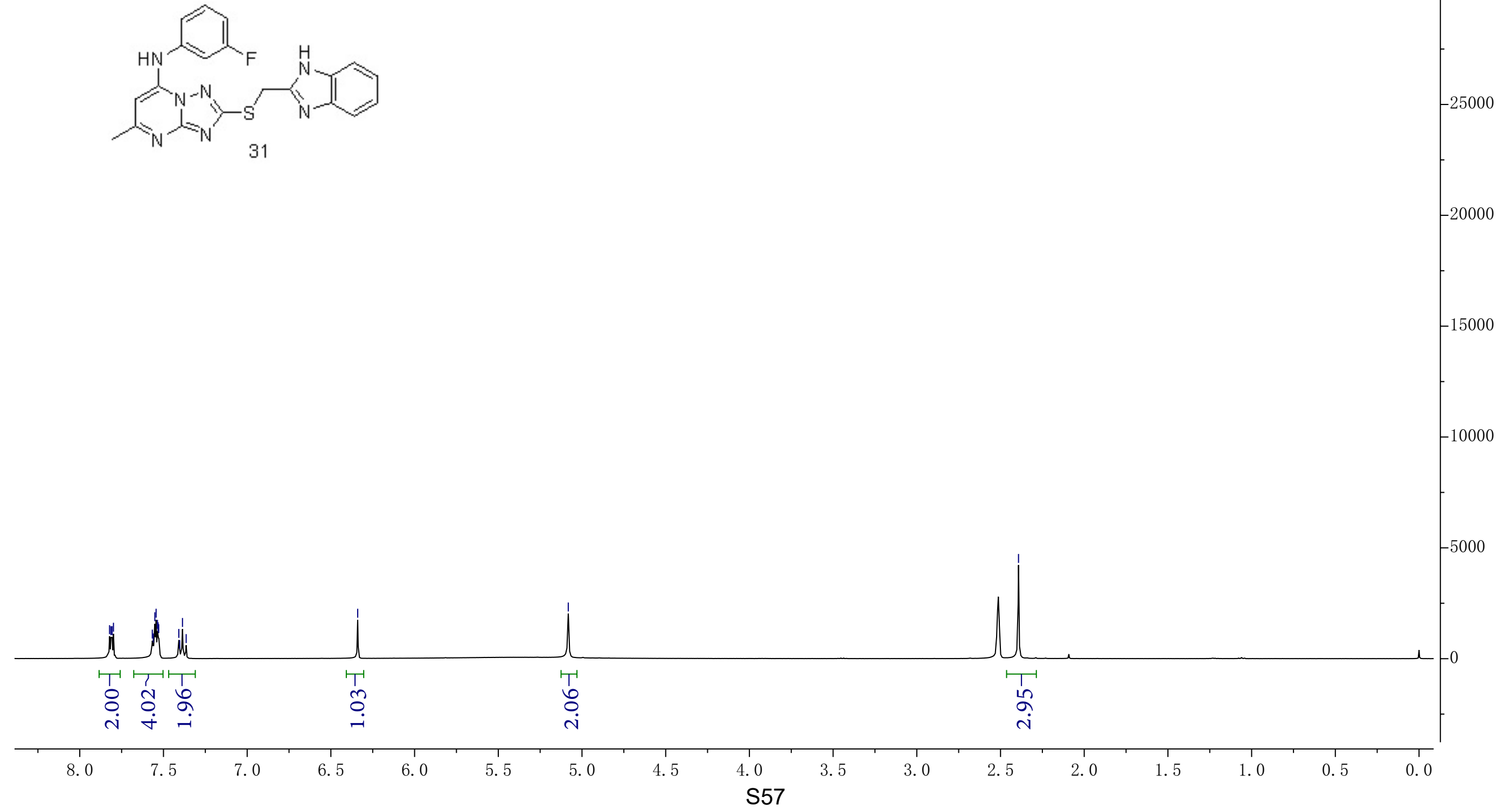

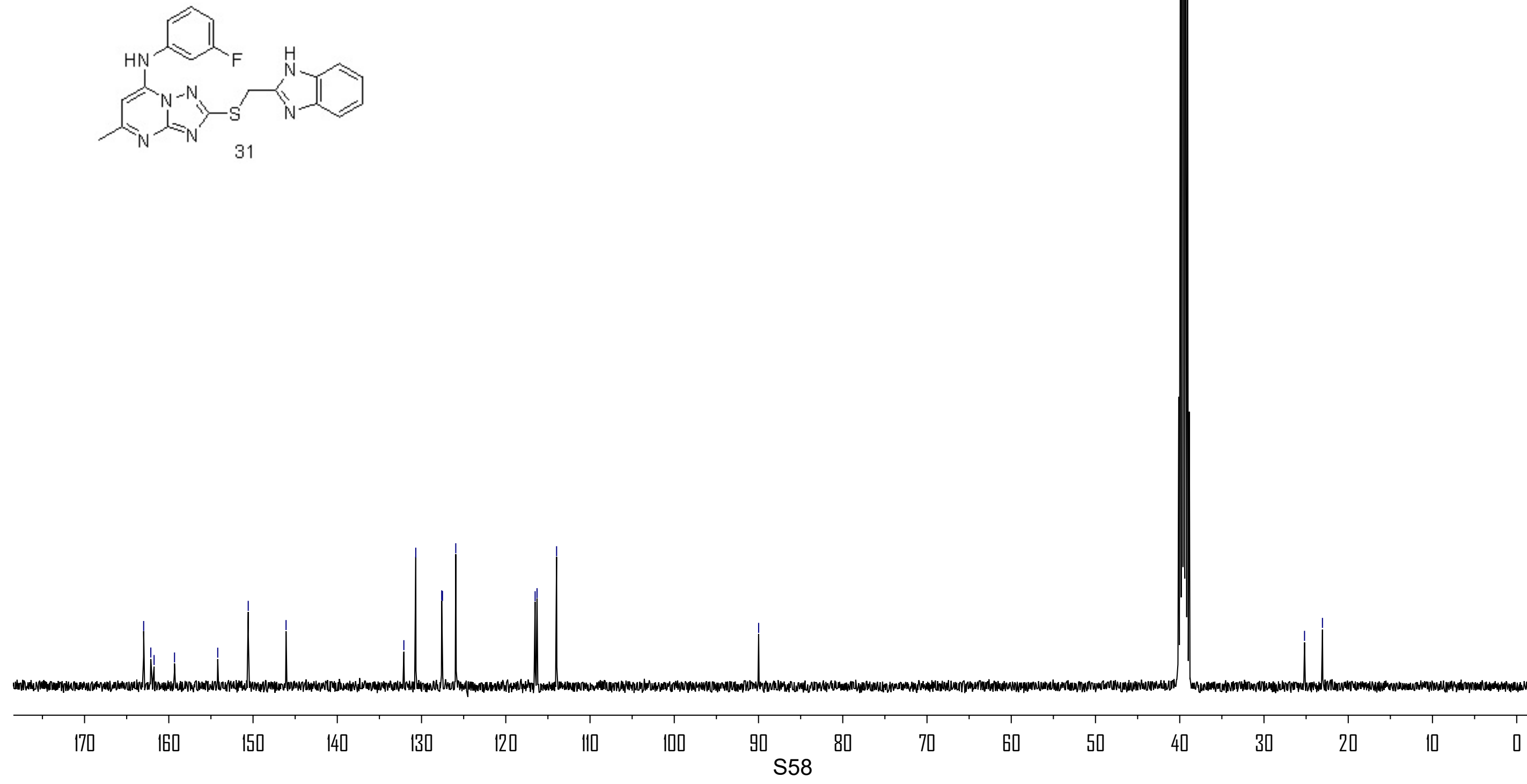

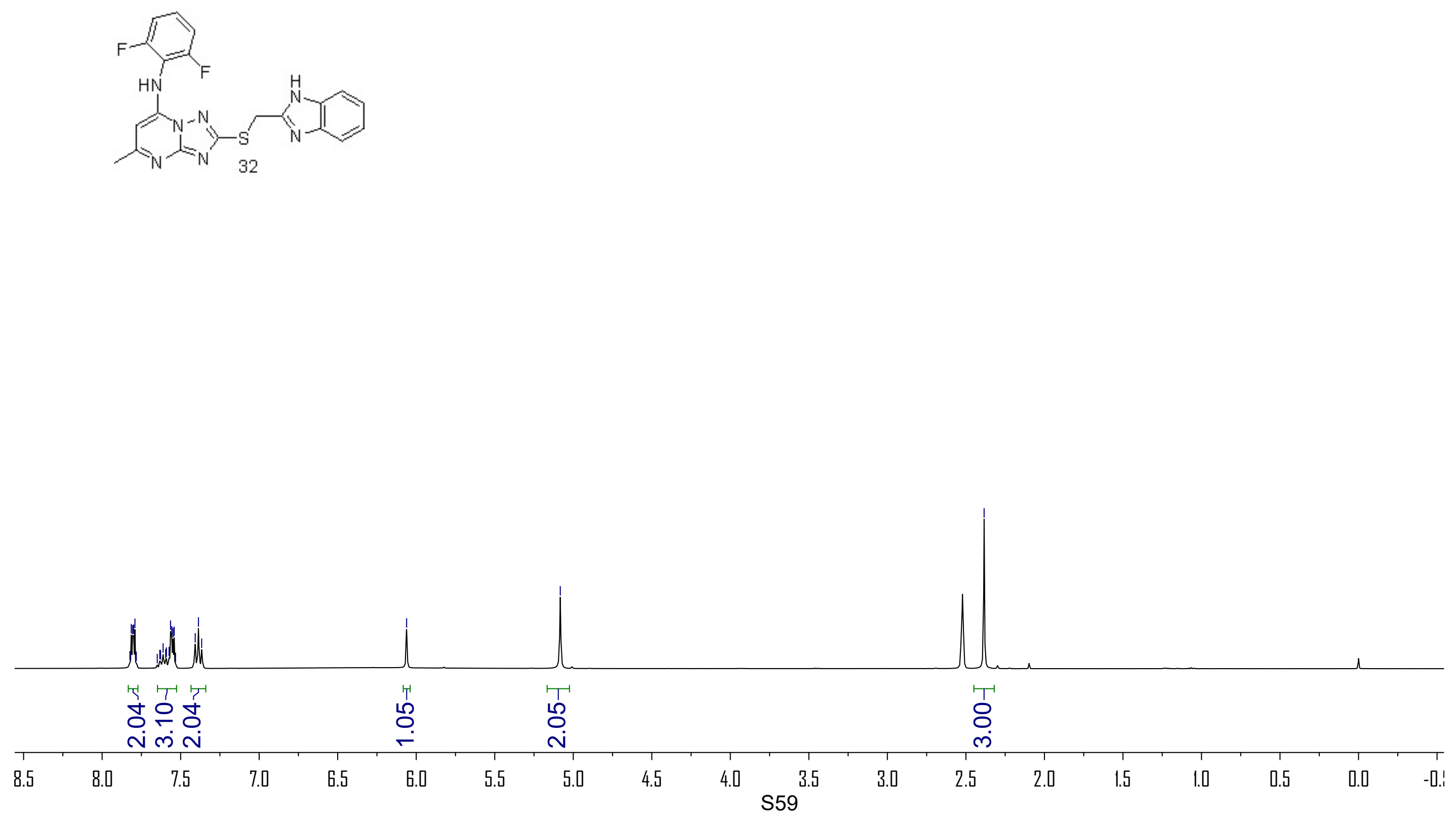

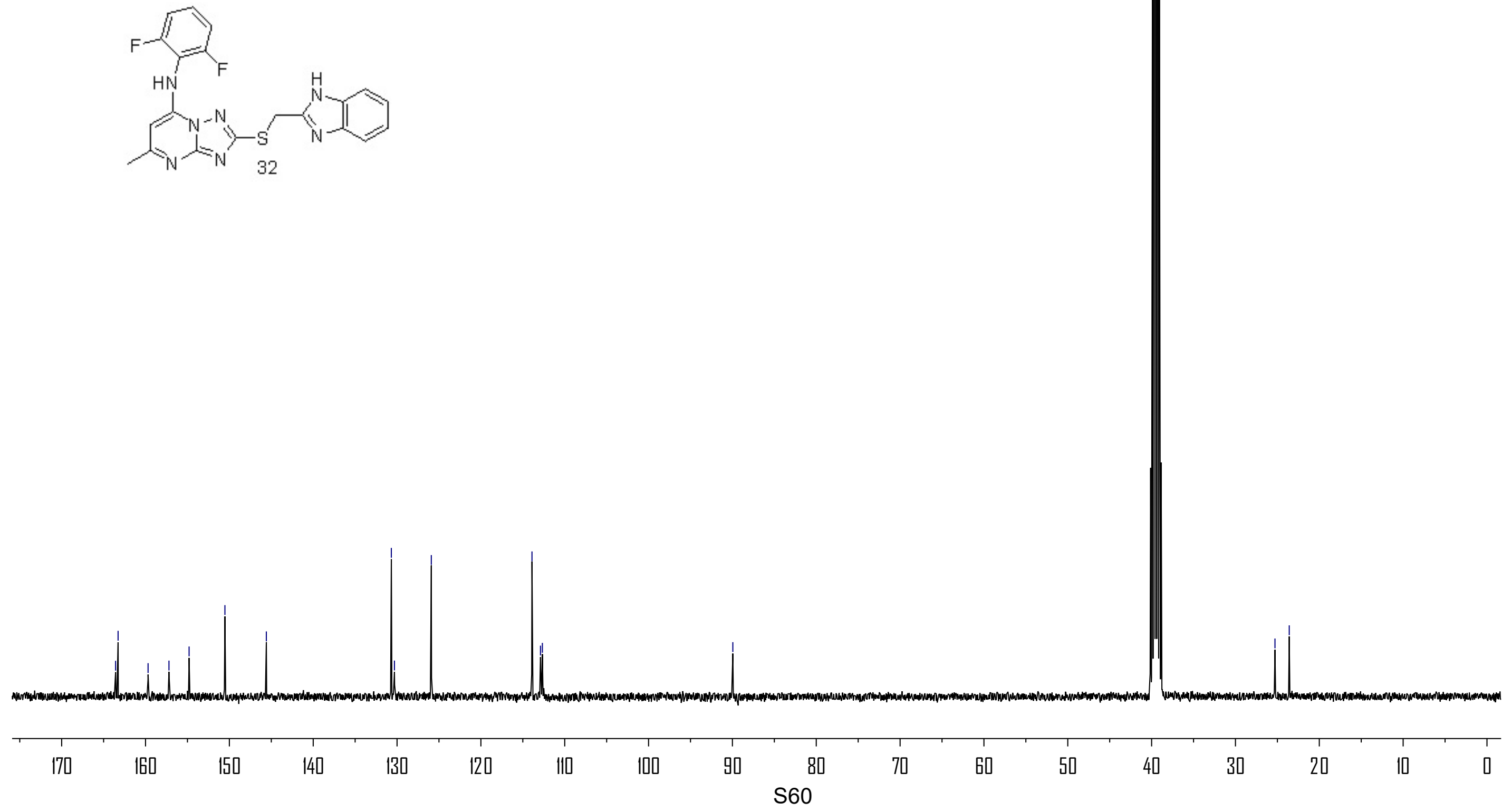

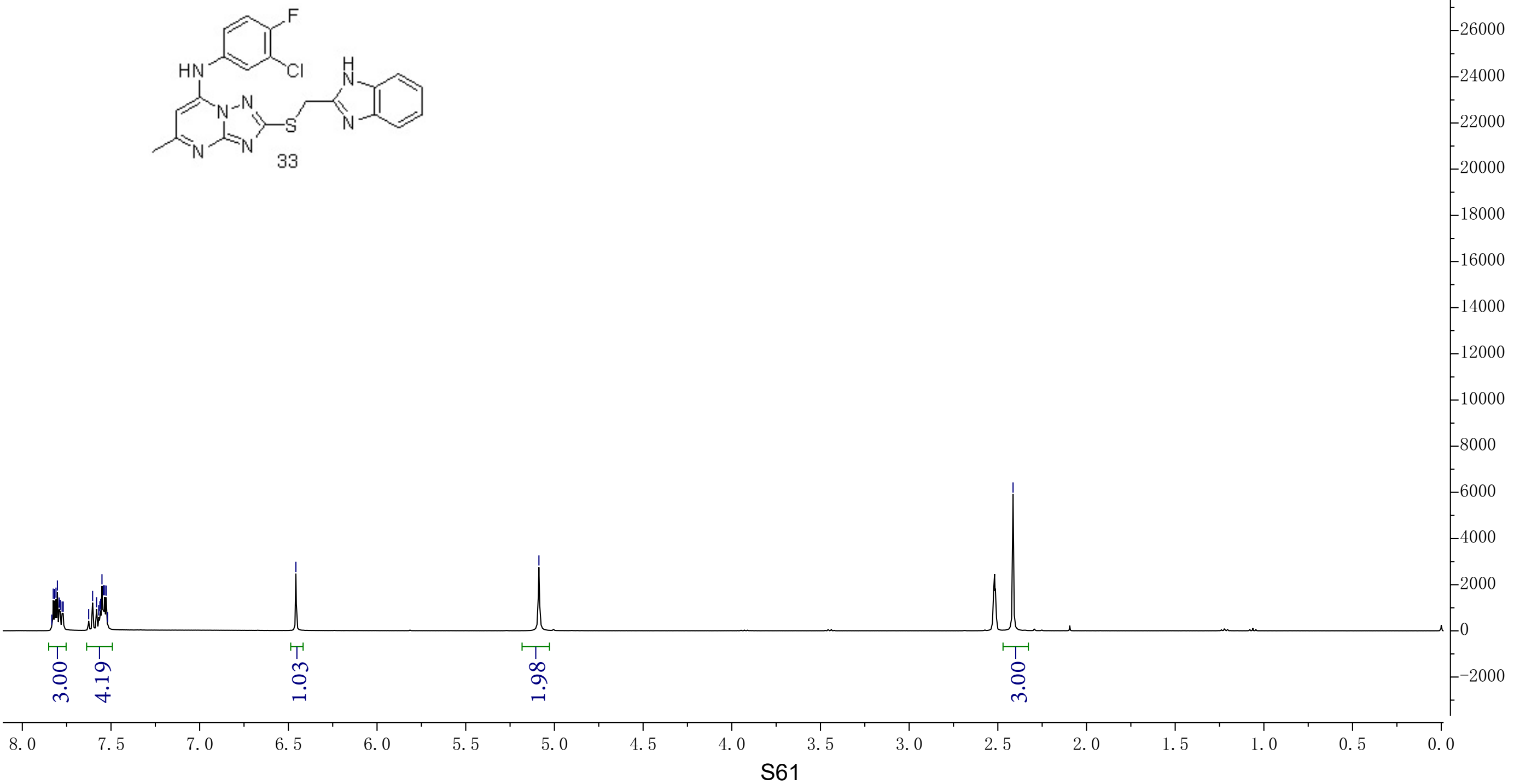

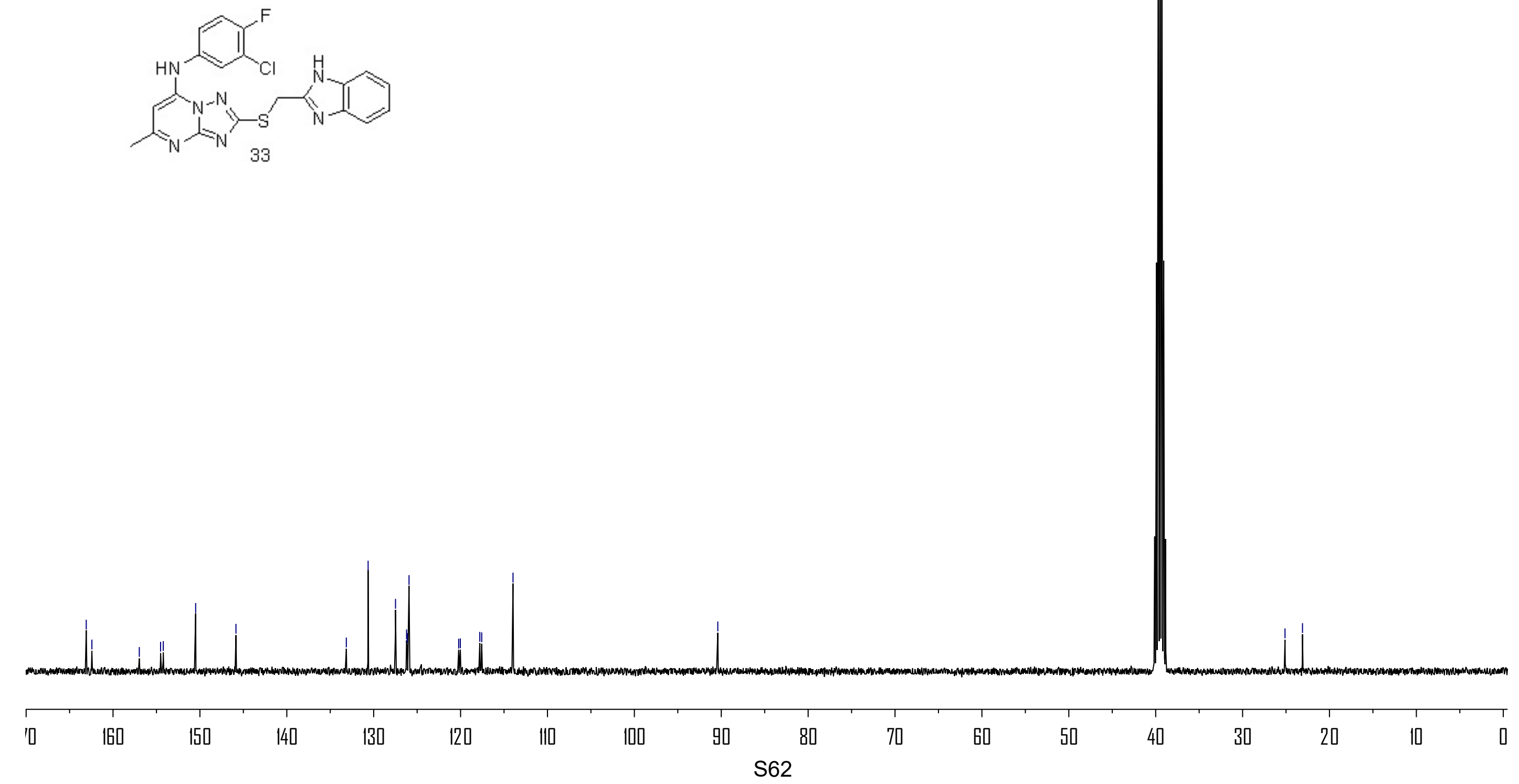
IIIm
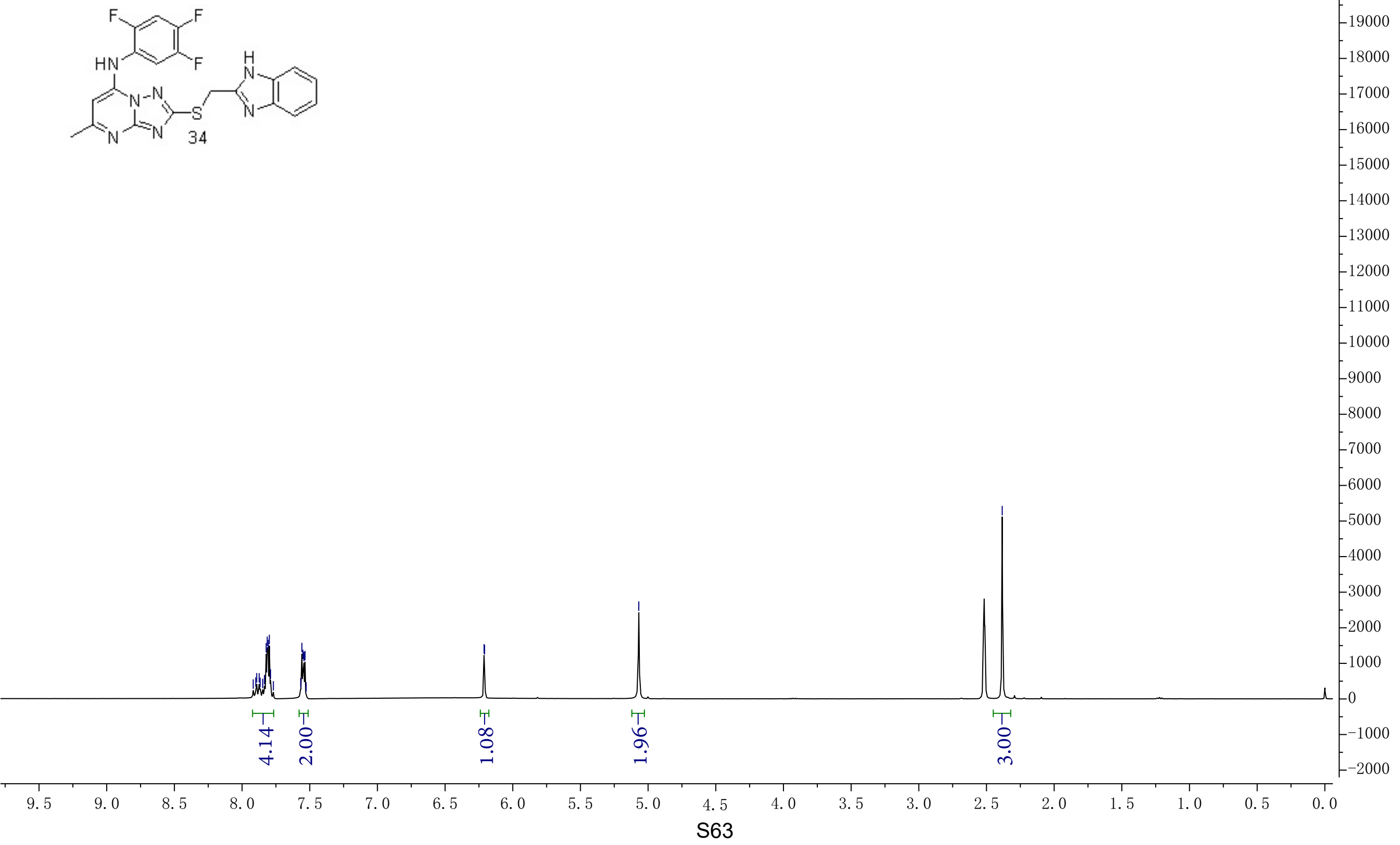


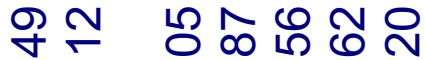

ชூ

广)

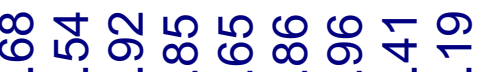

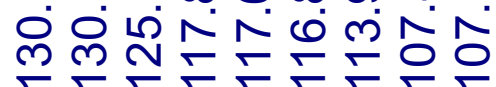

穴宁示宁

ల

요

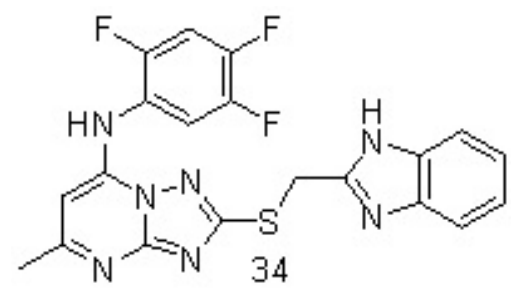

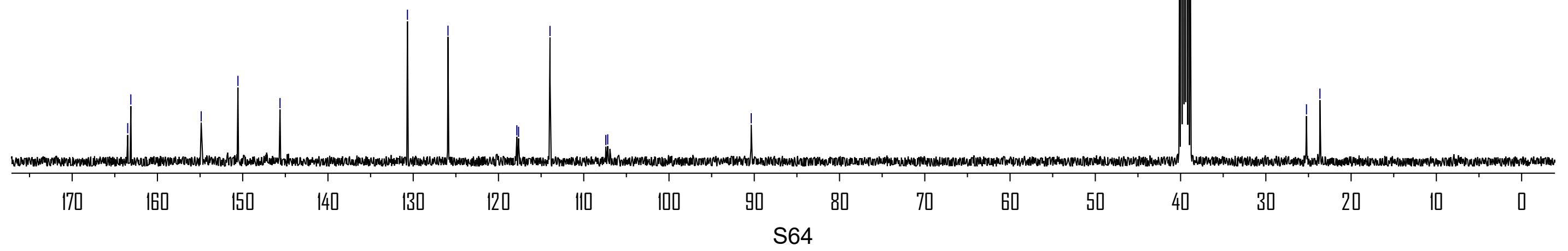



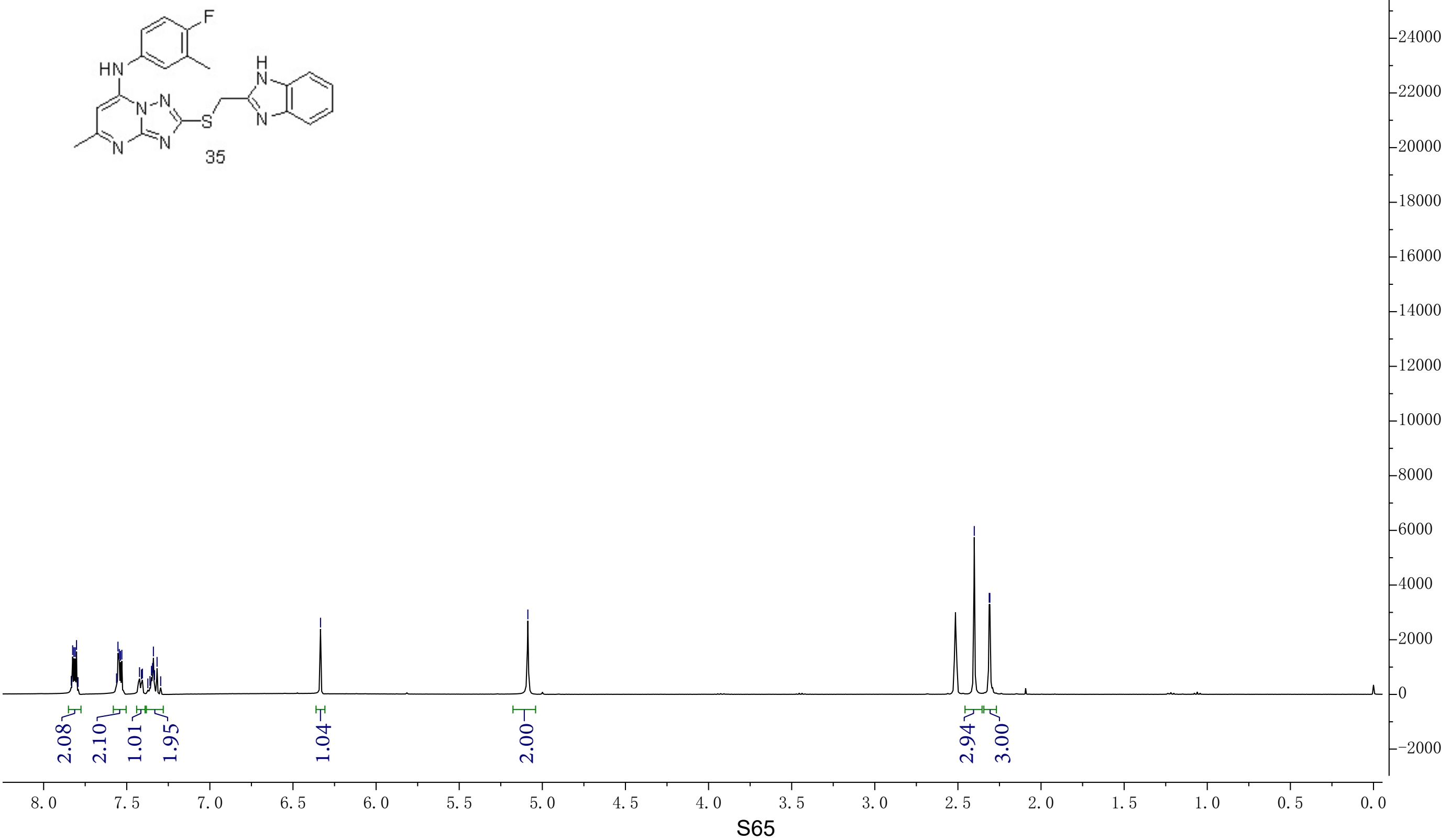


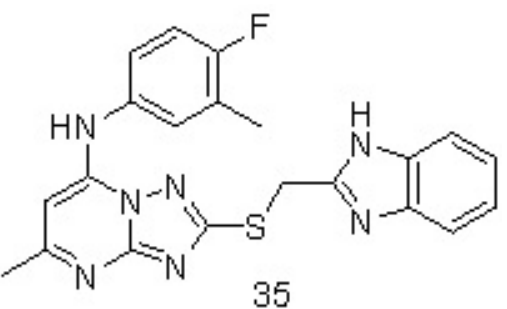



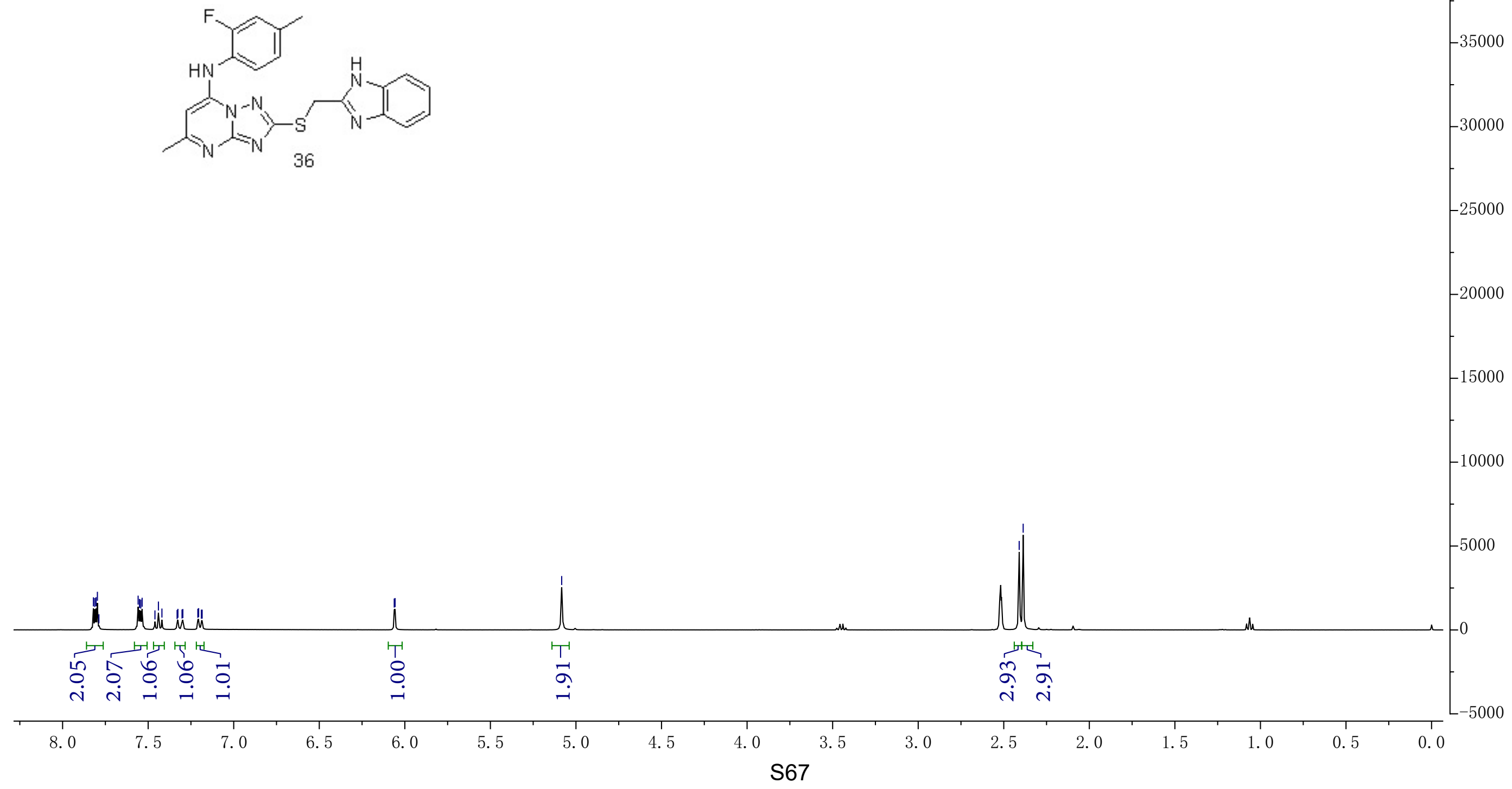


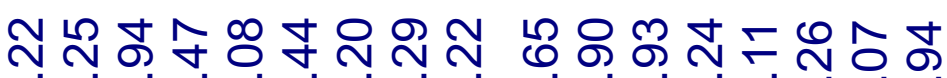

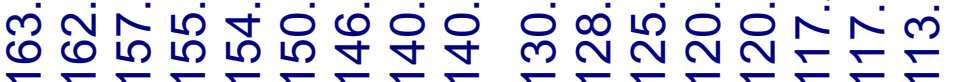

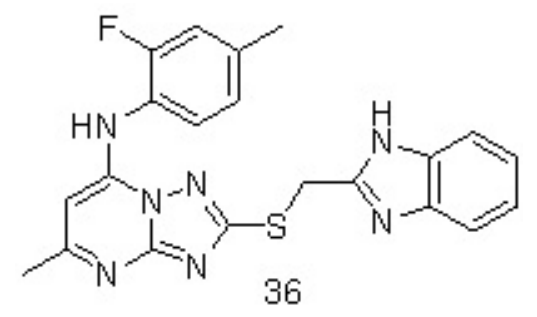




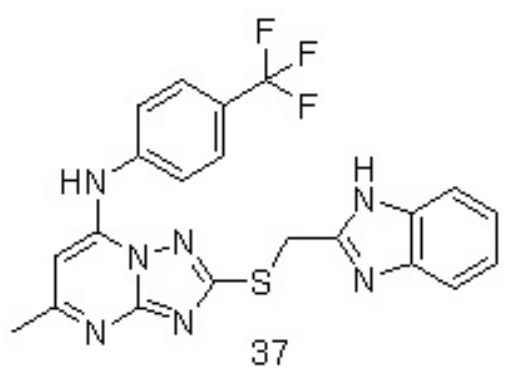



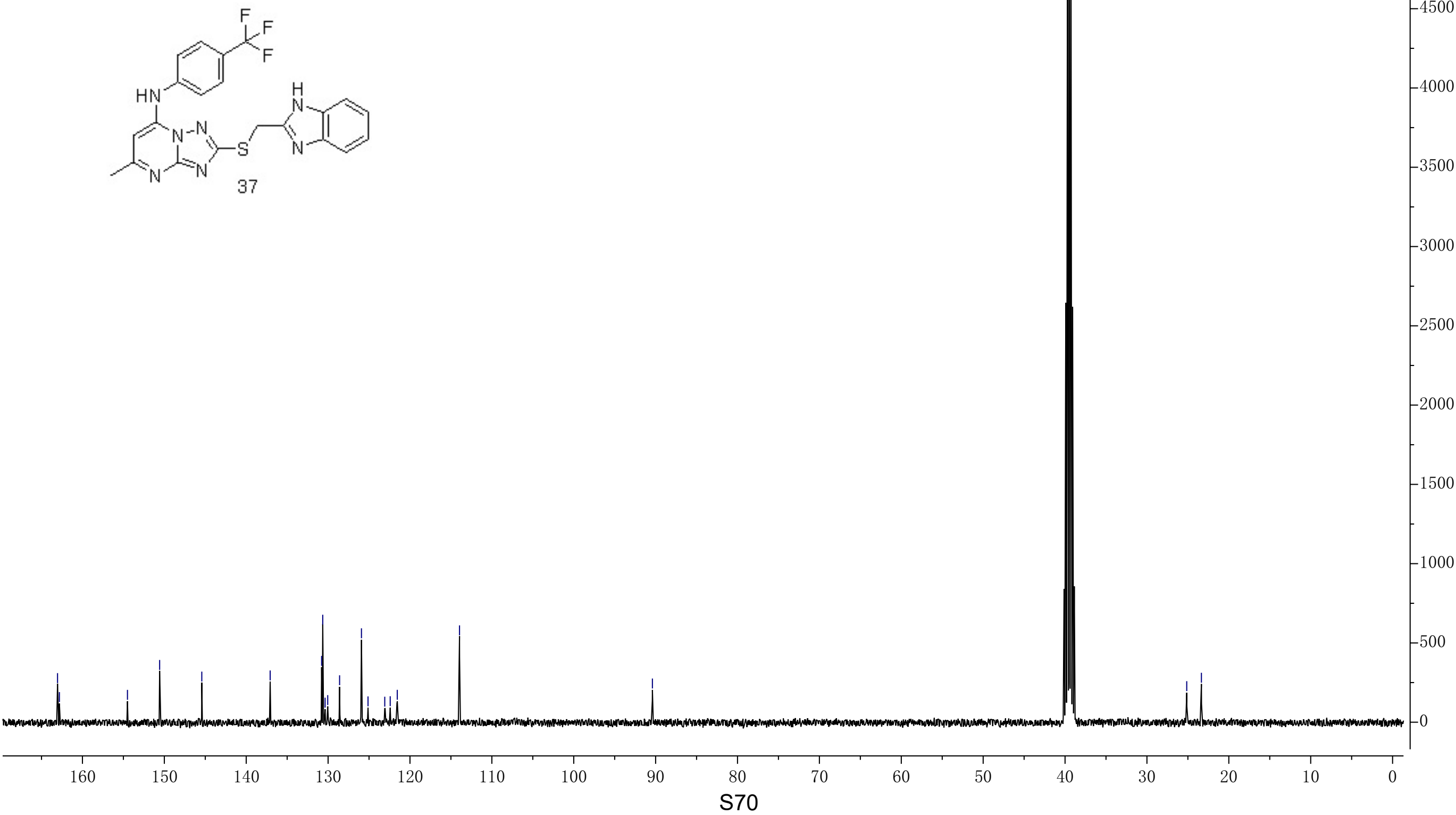

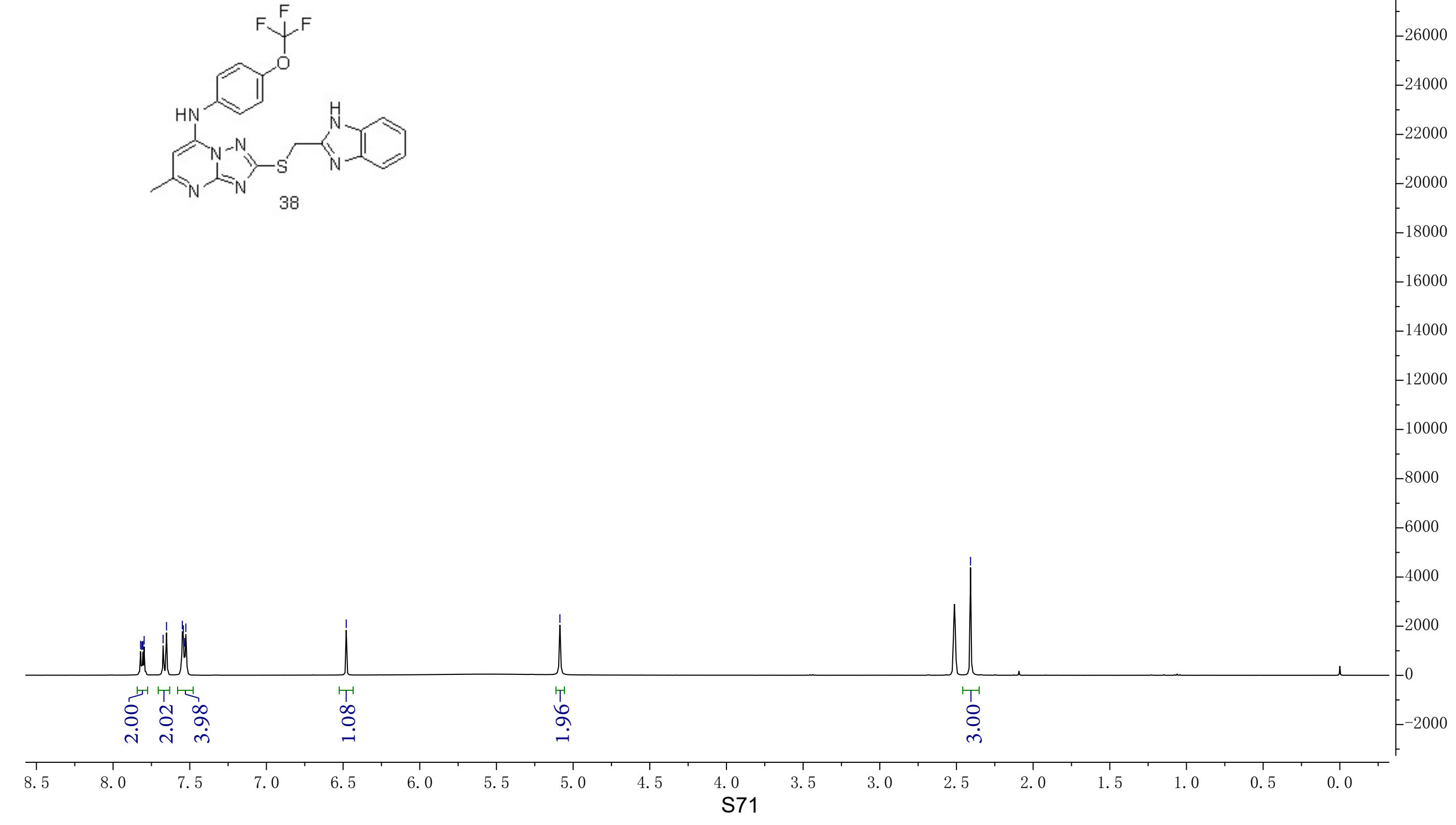

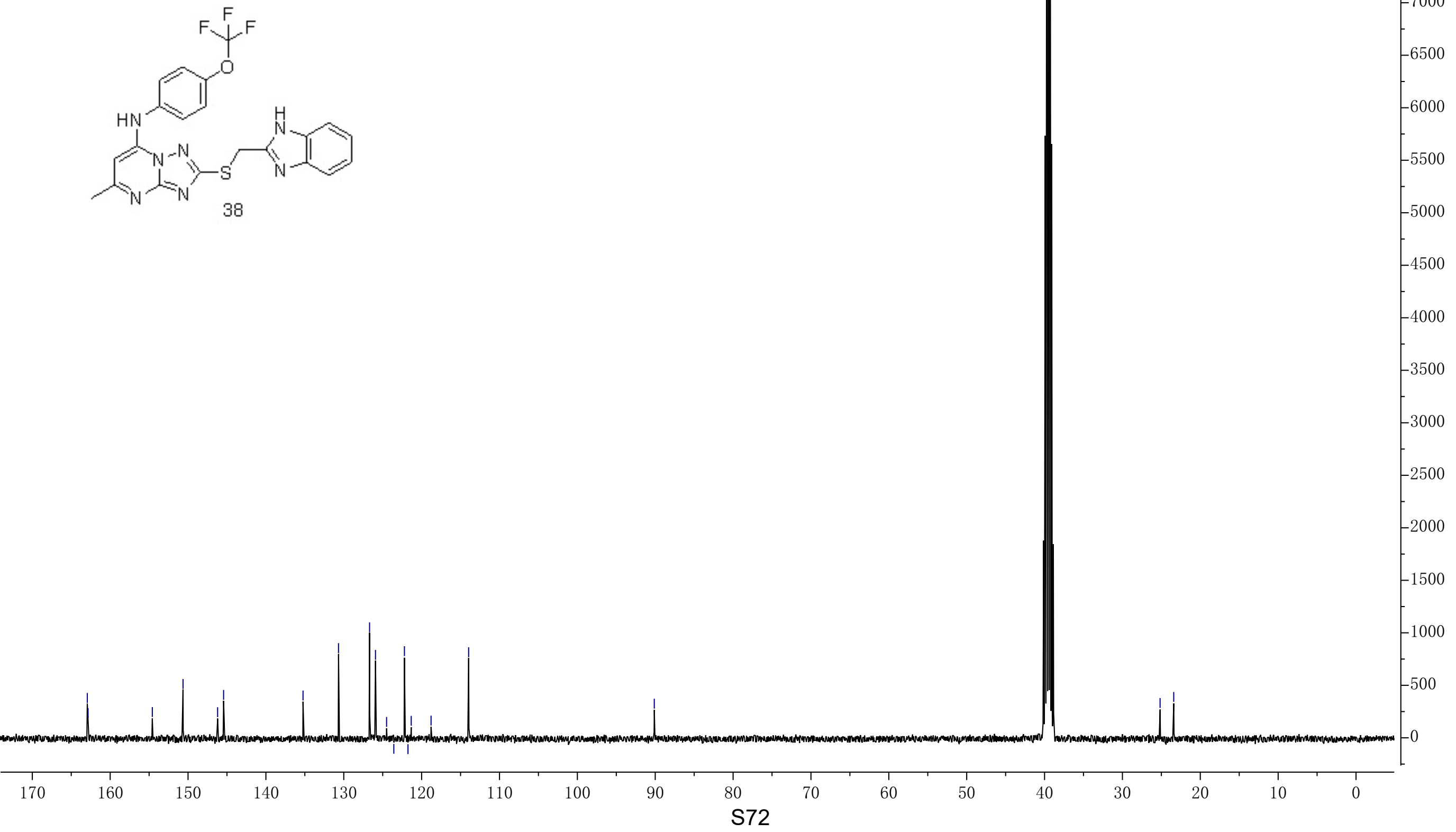

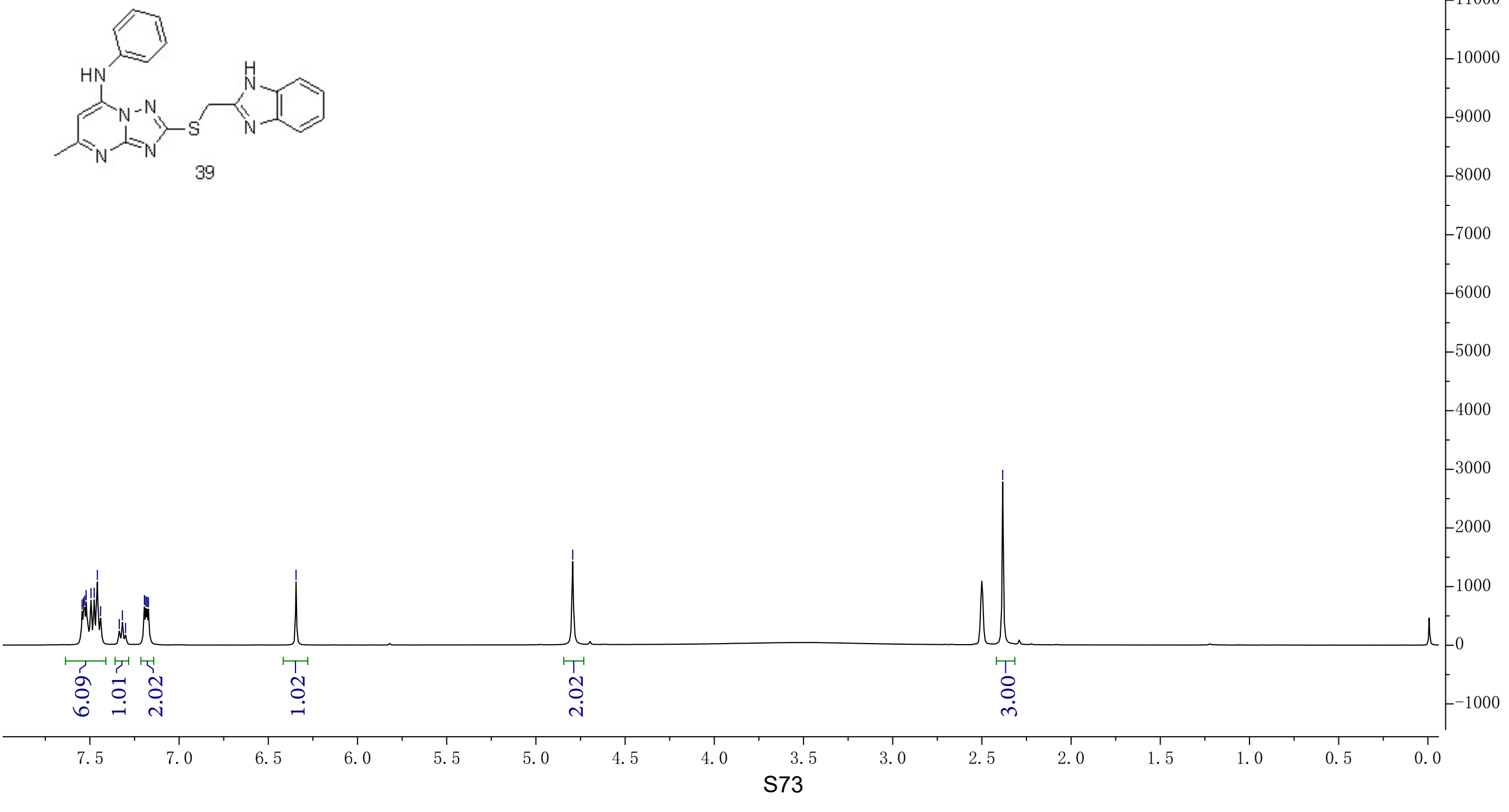

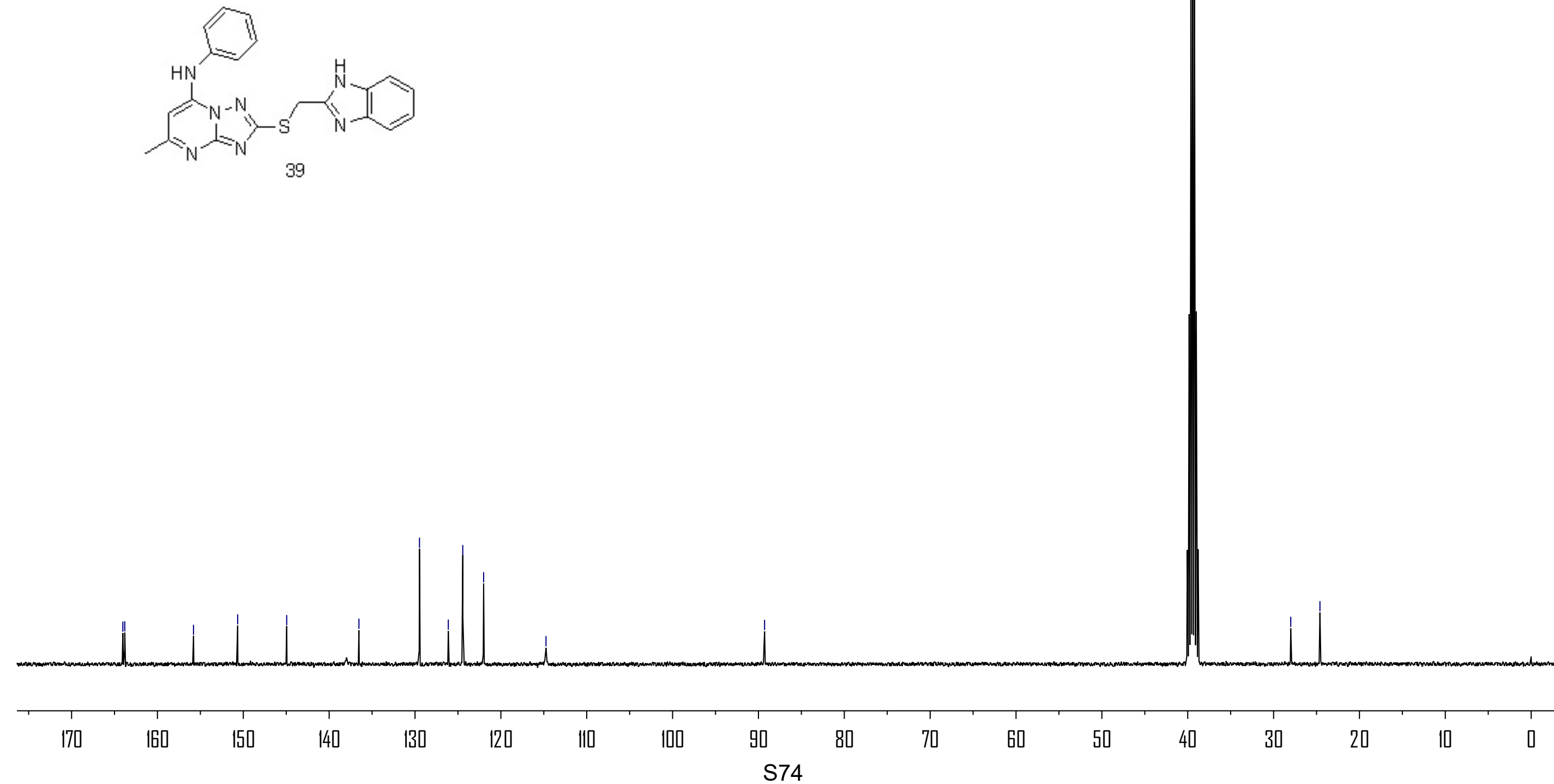

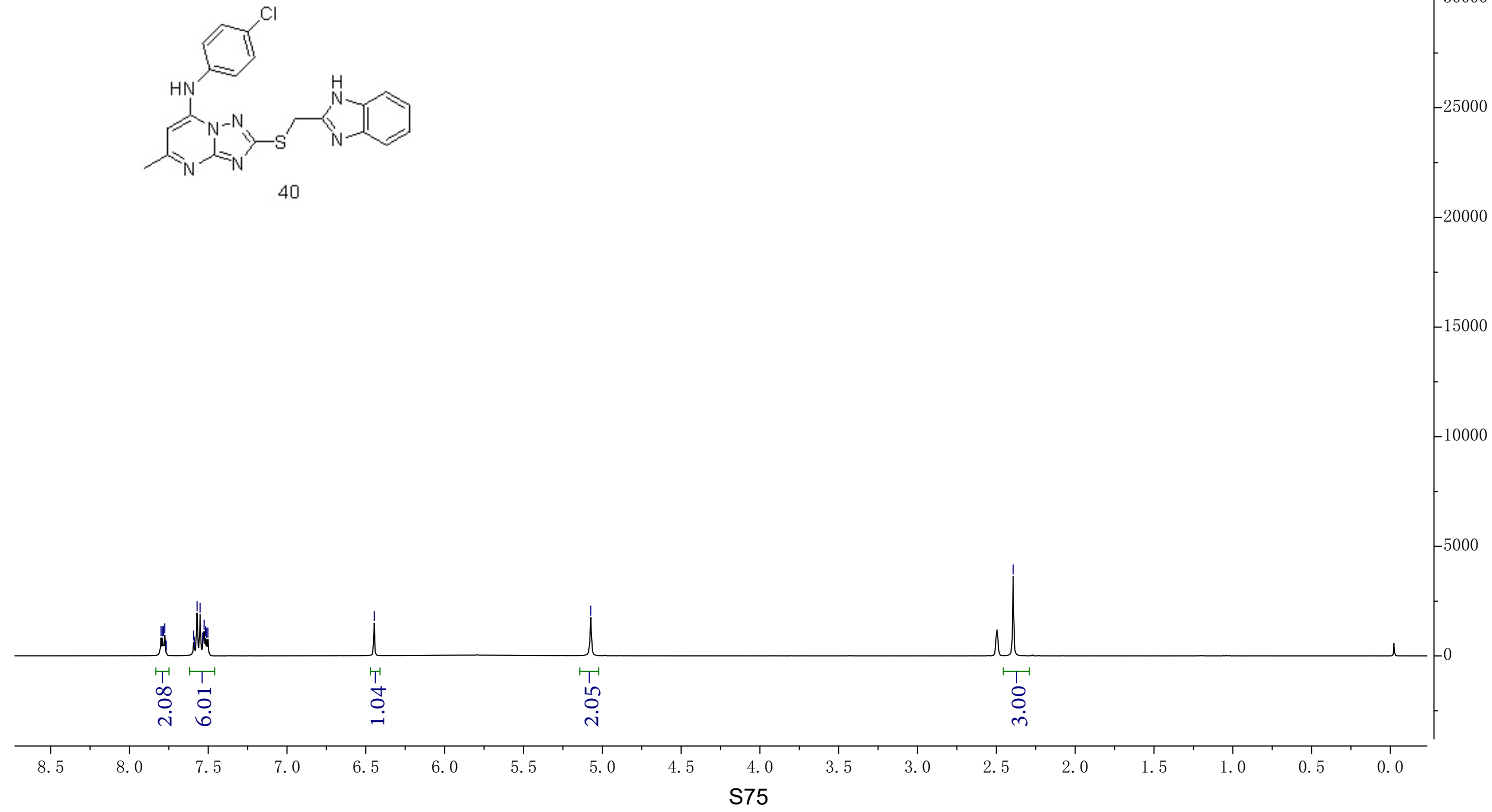


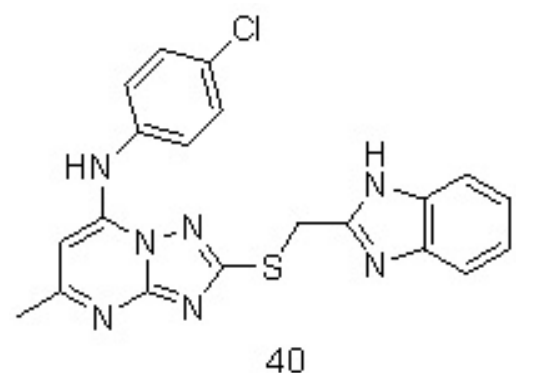

لنس

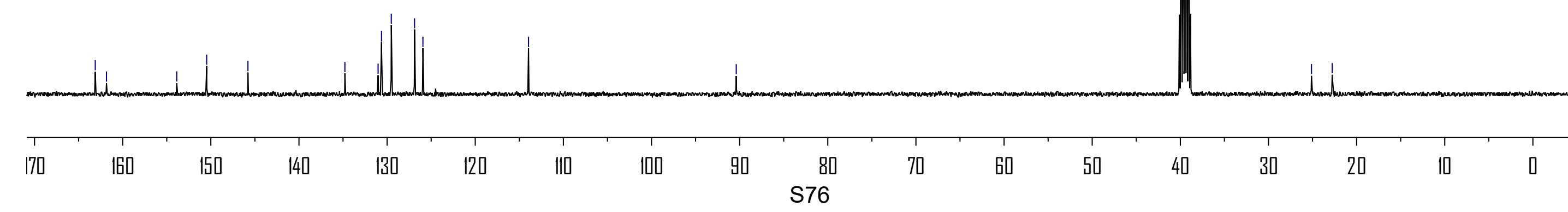



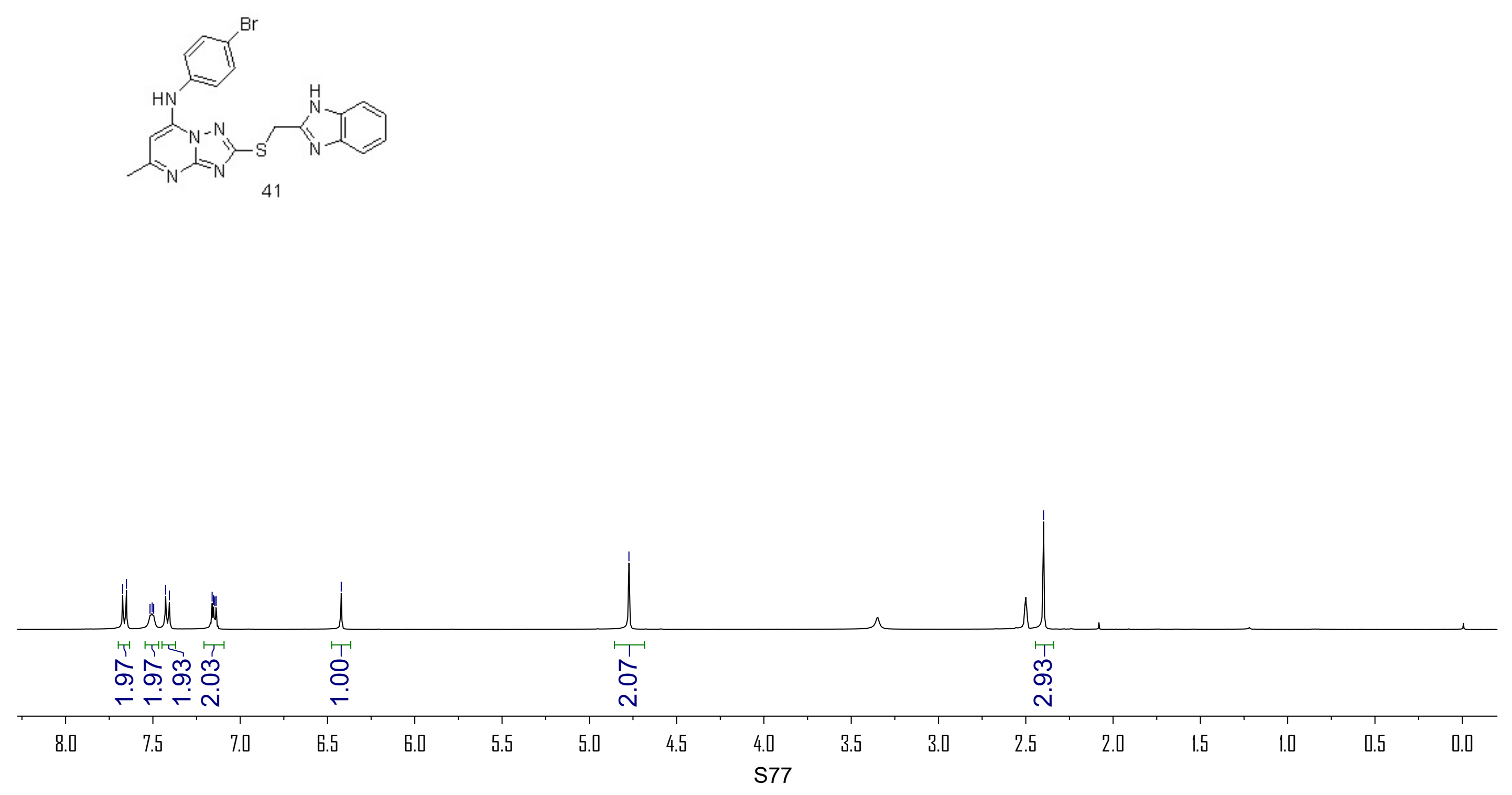

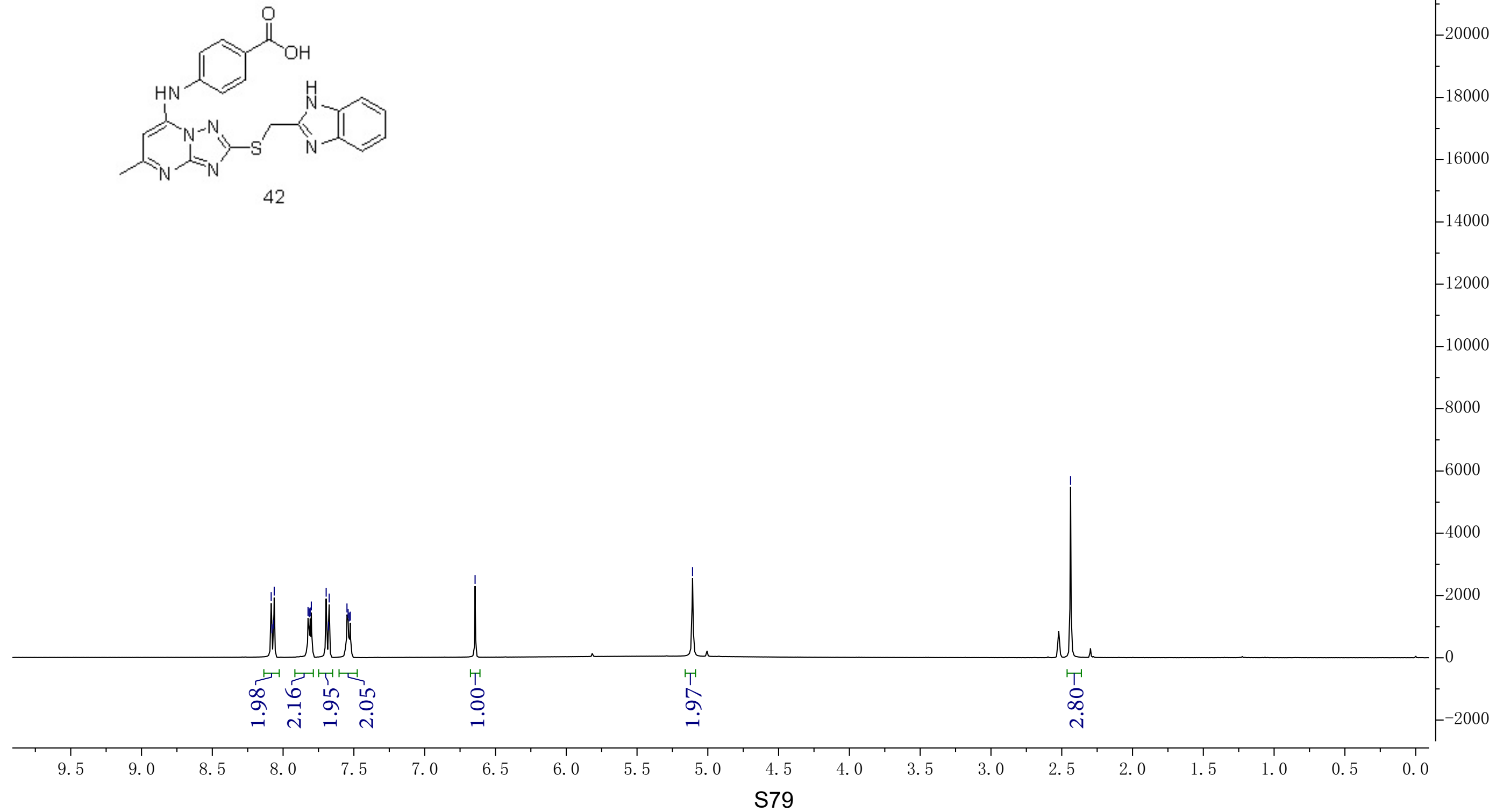

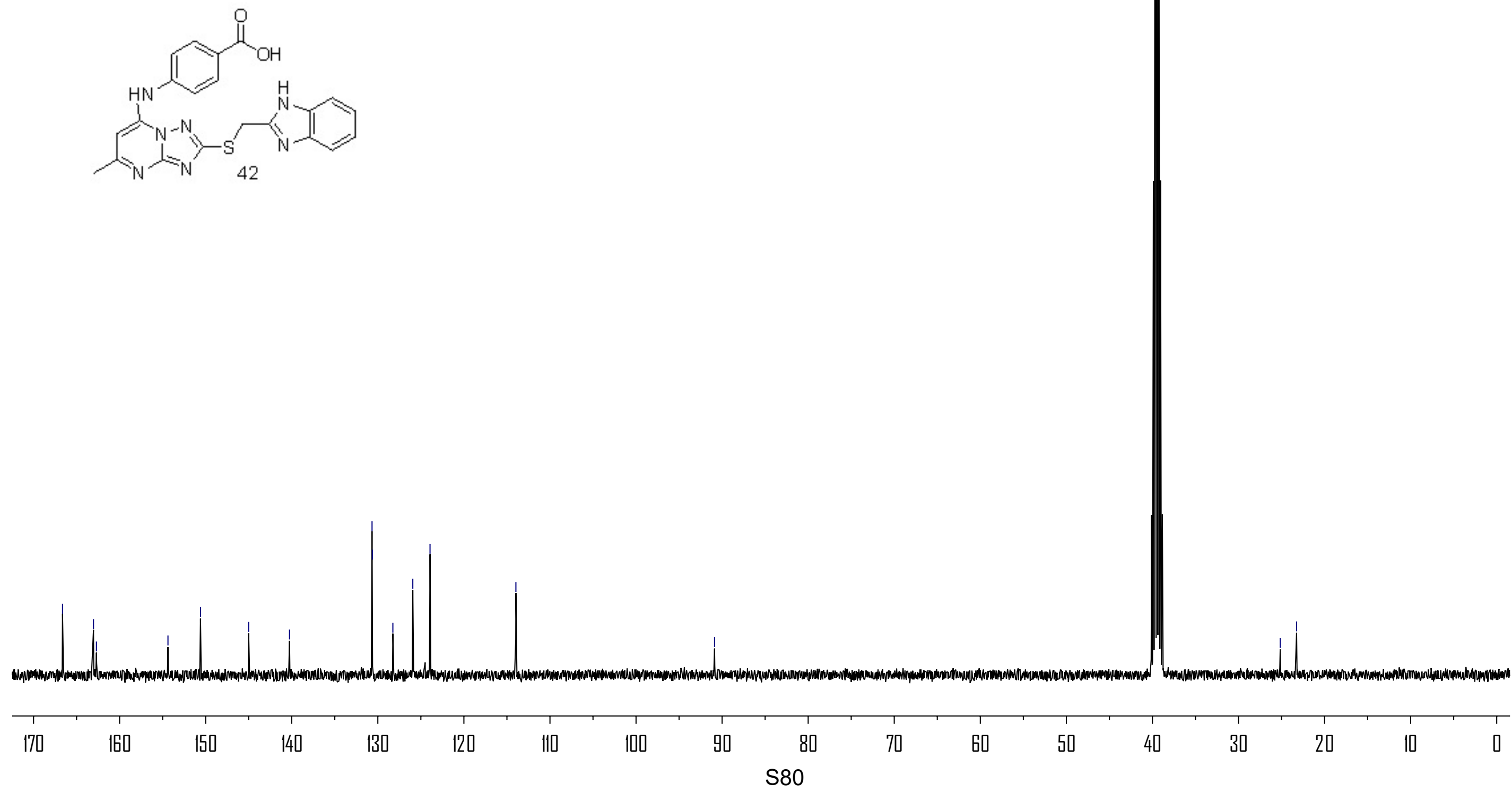

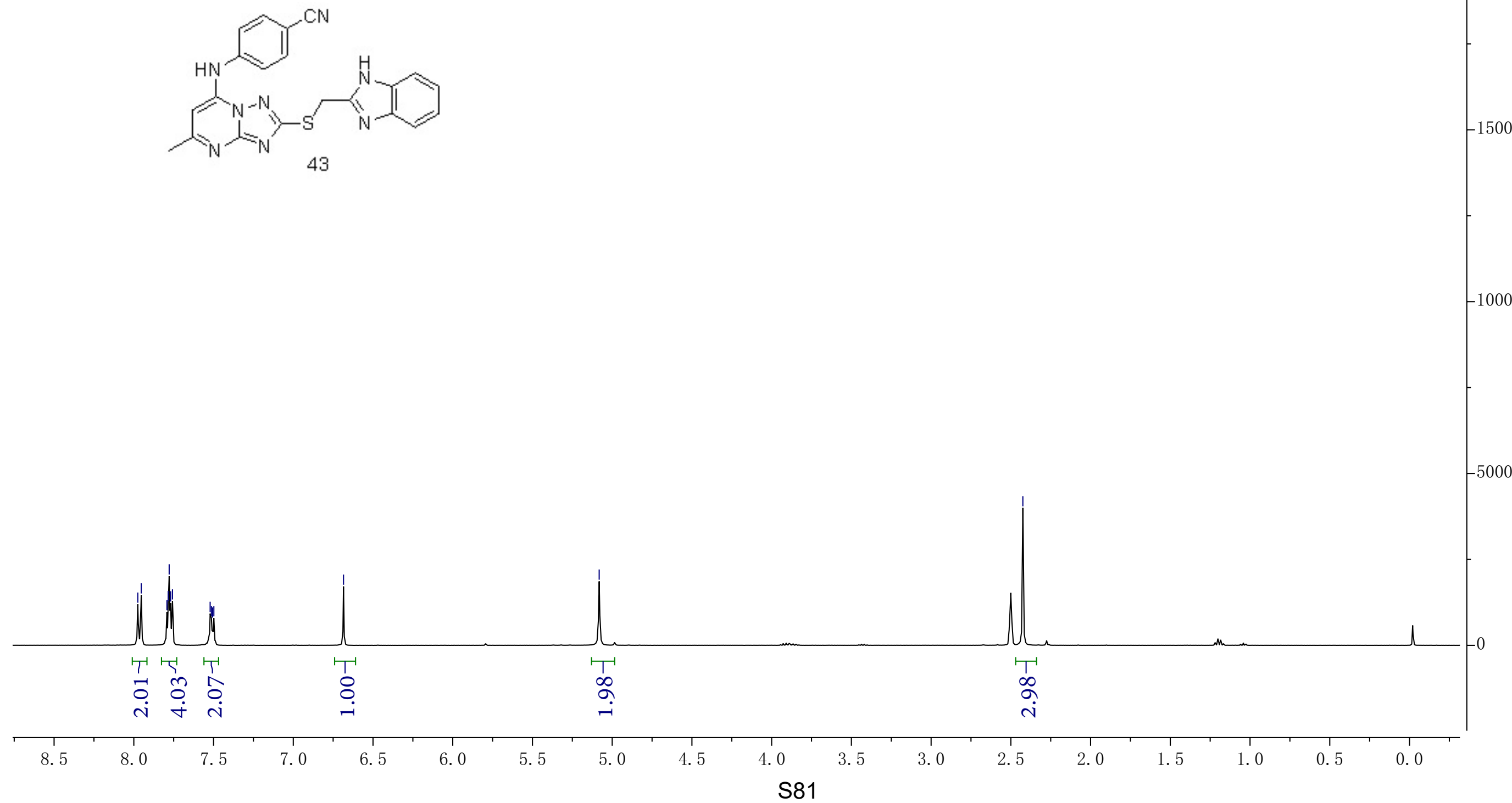
ठ

ஸें

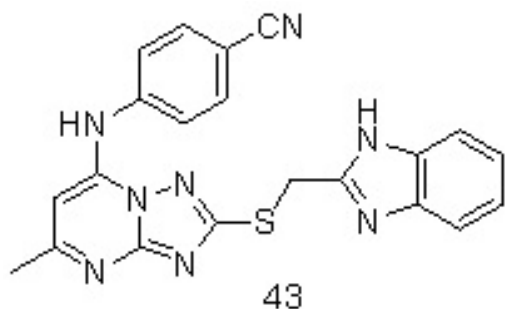

43 

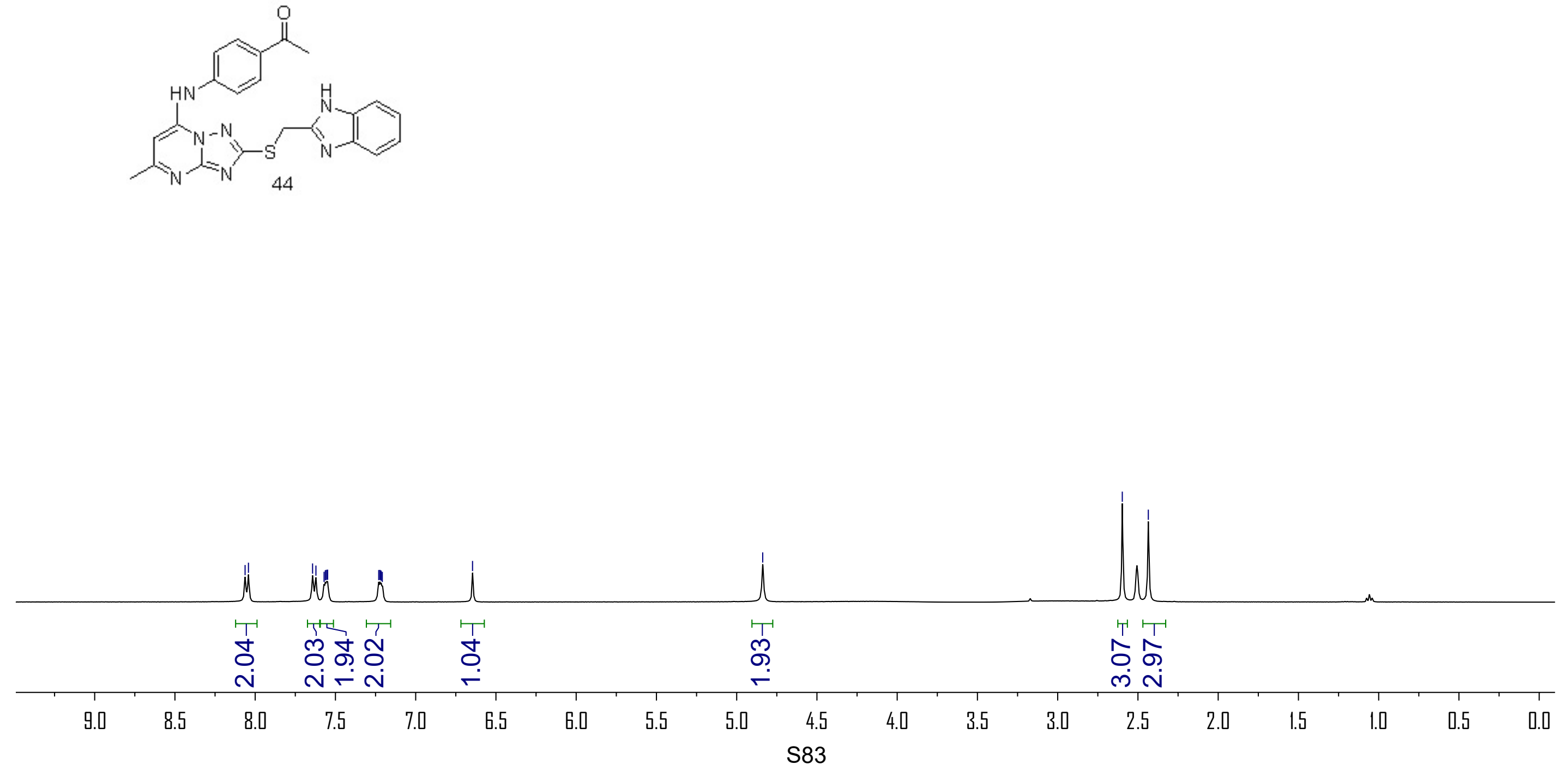


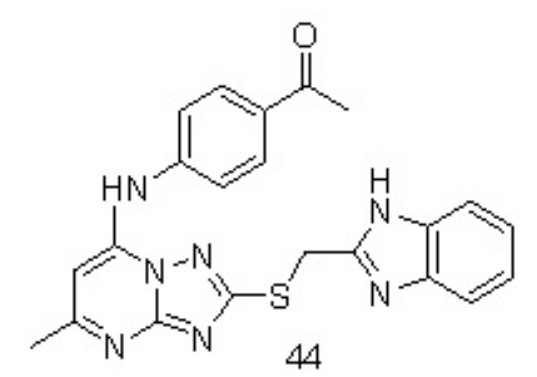

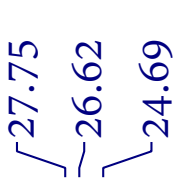



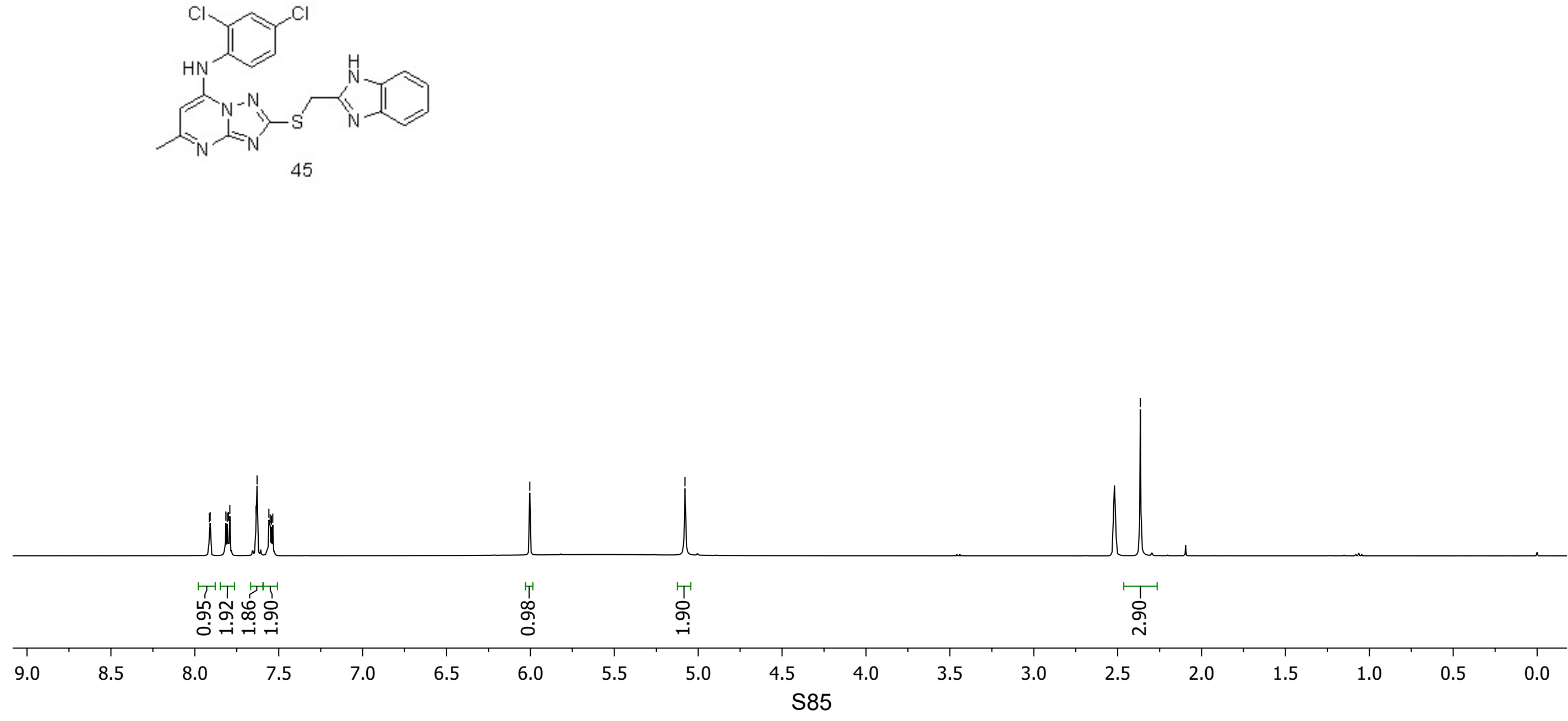

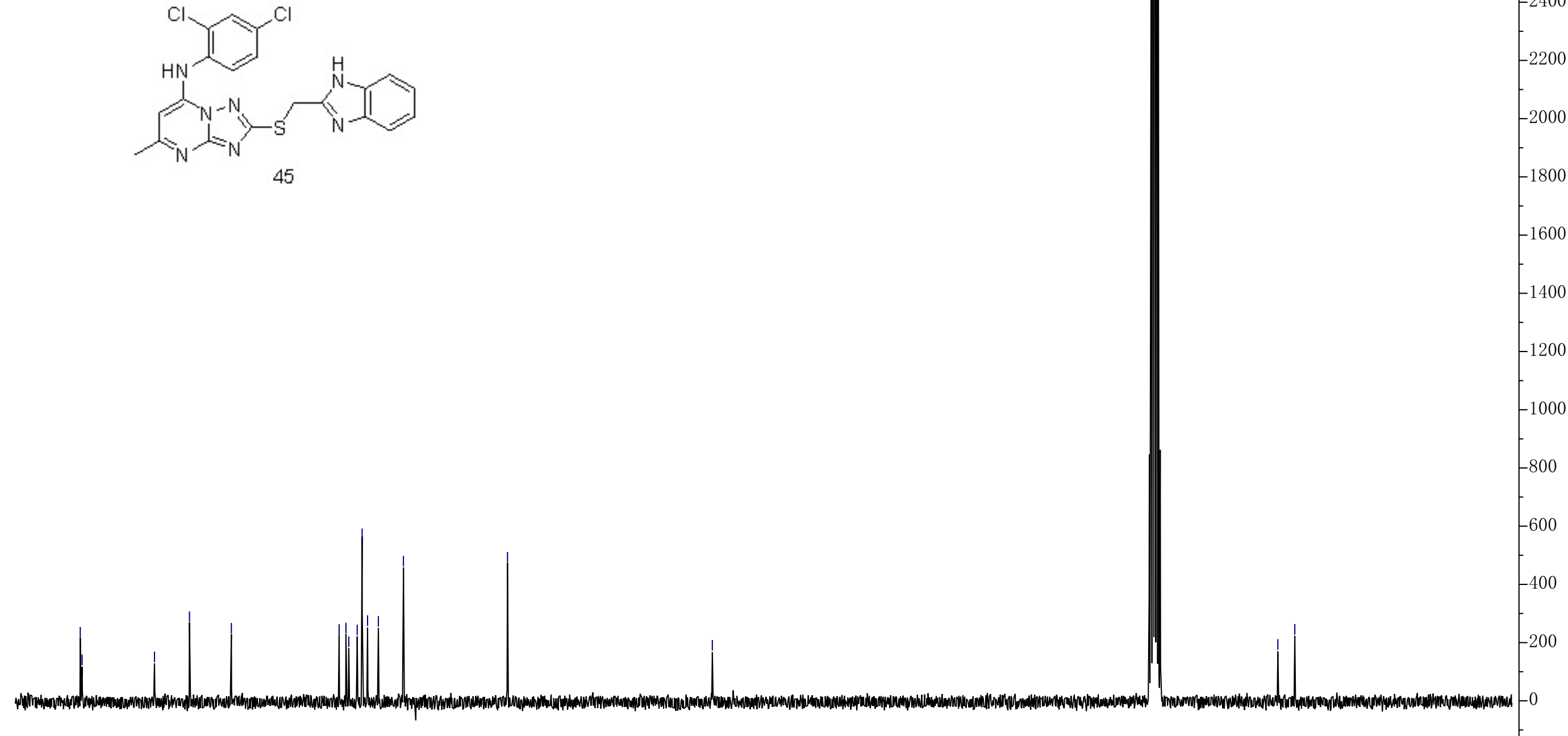

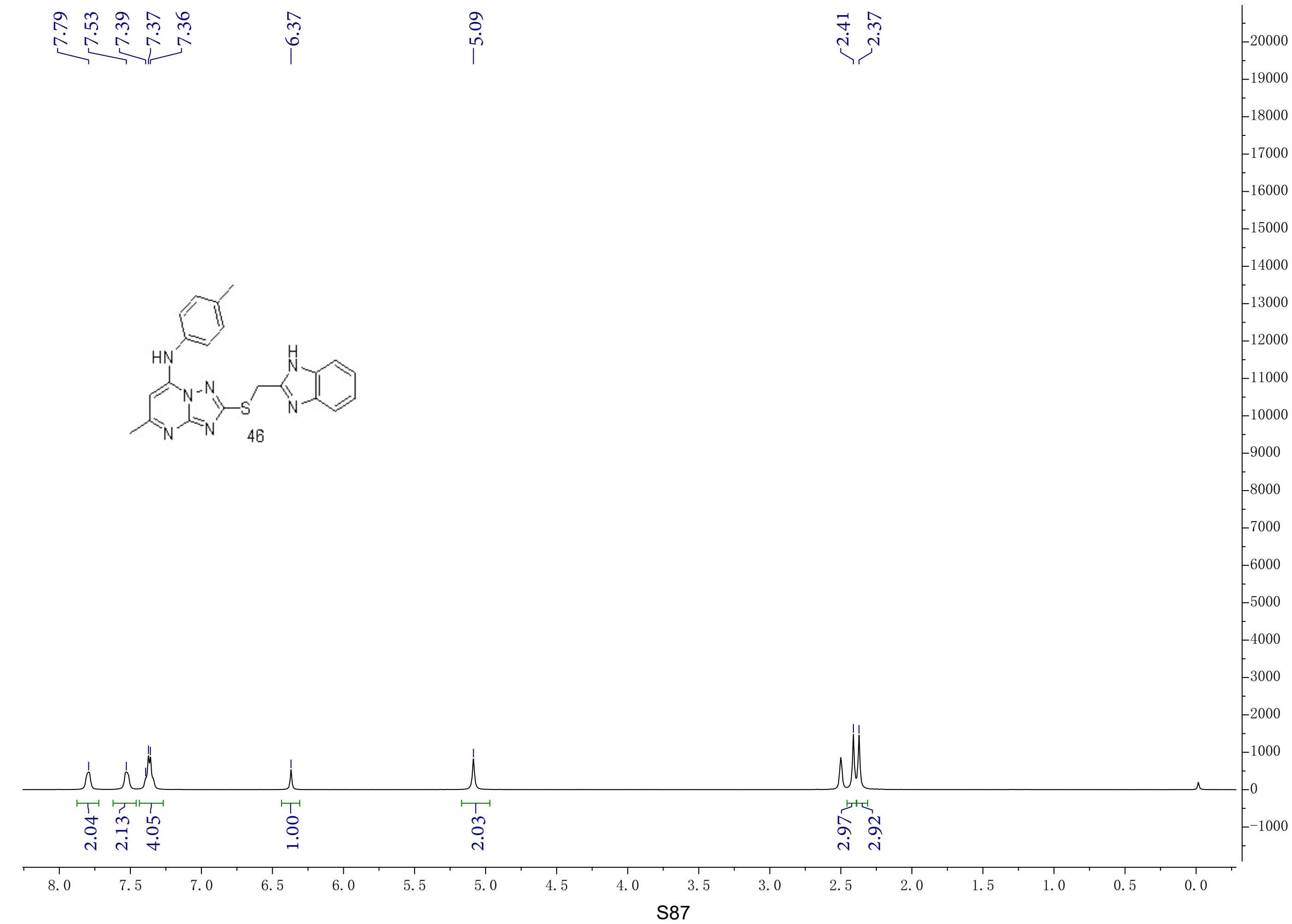


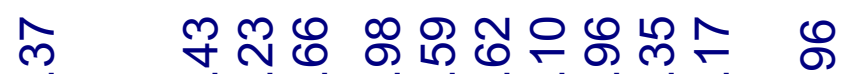

ஜ $\quad$ ஸे
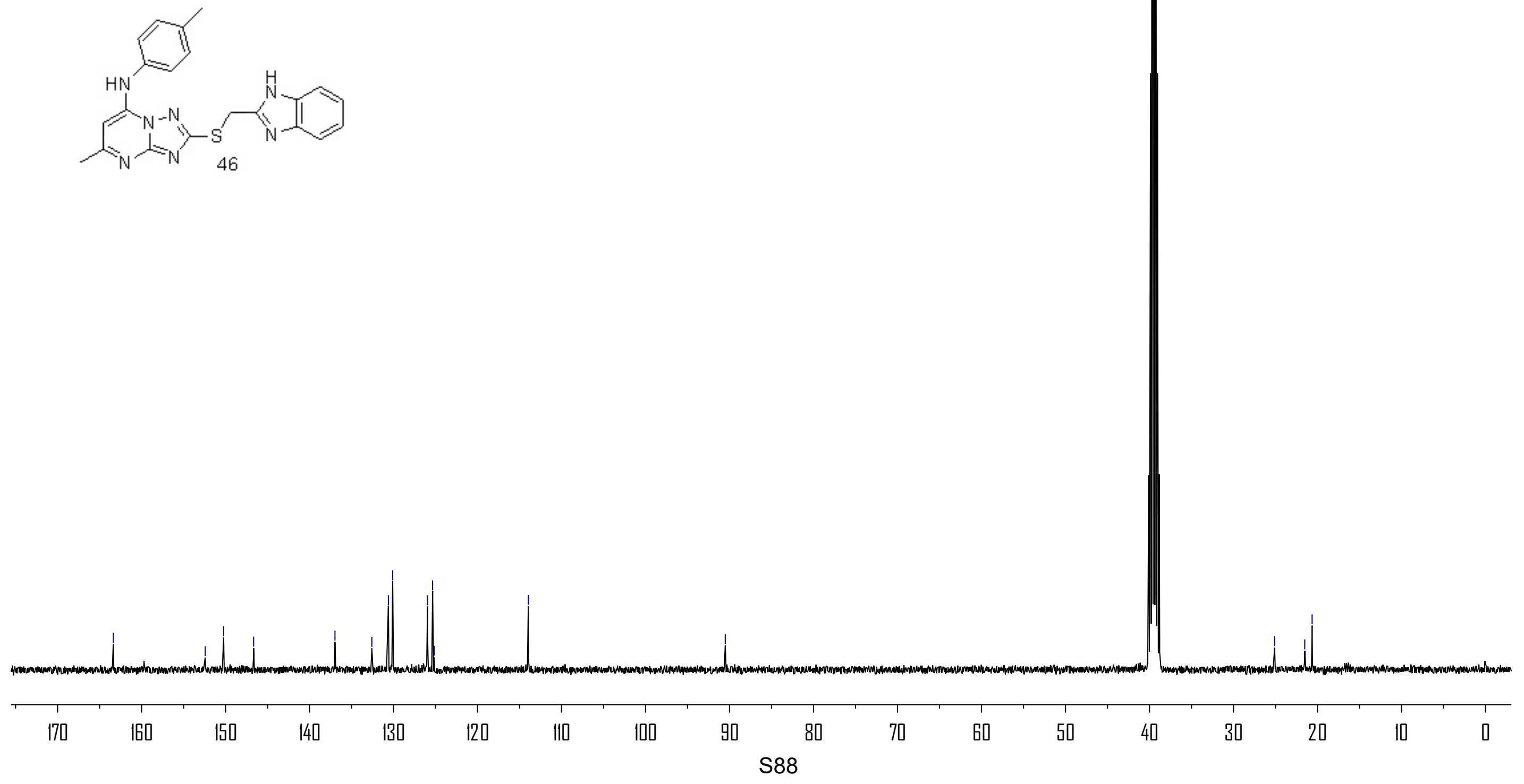

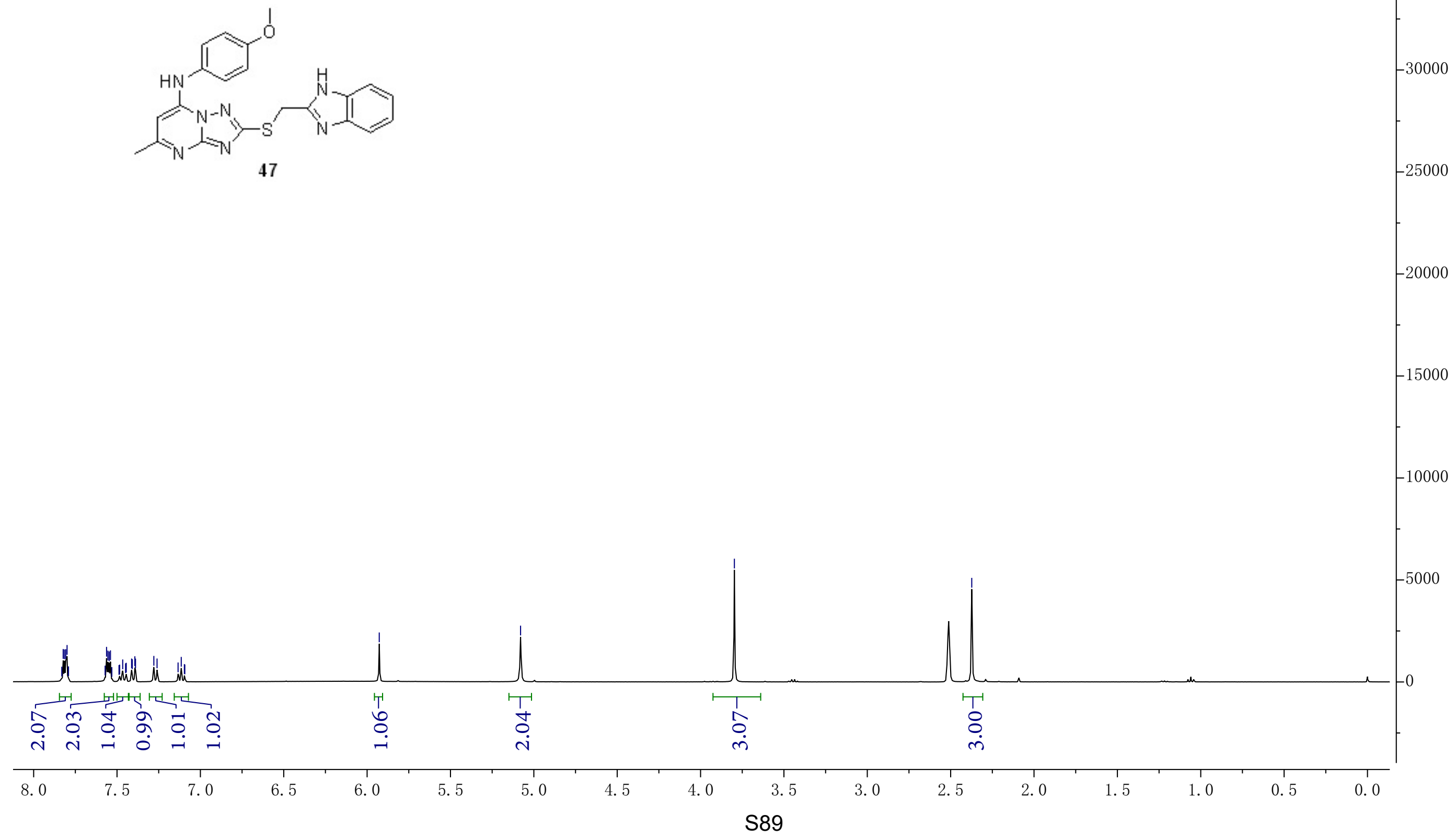

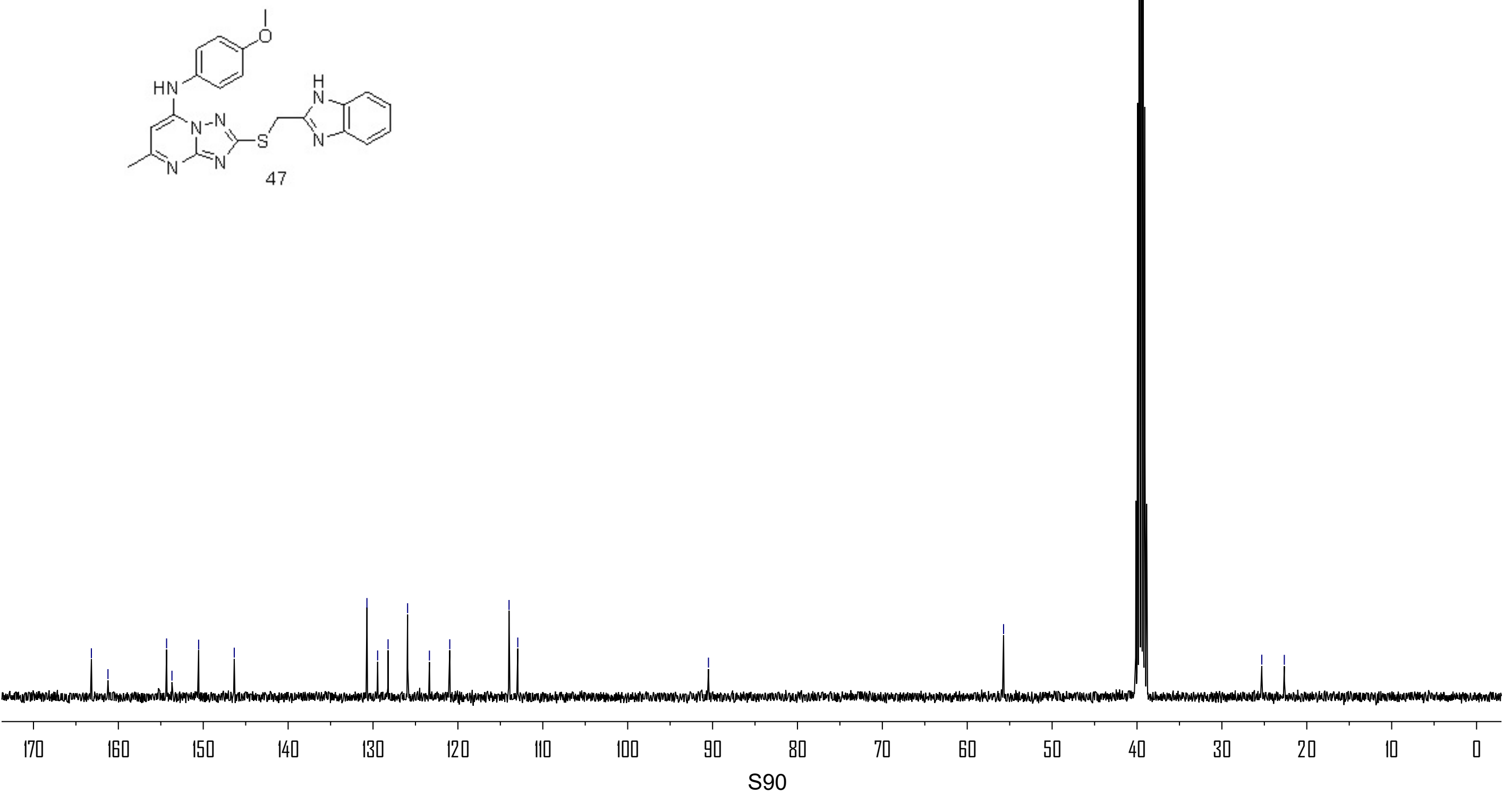

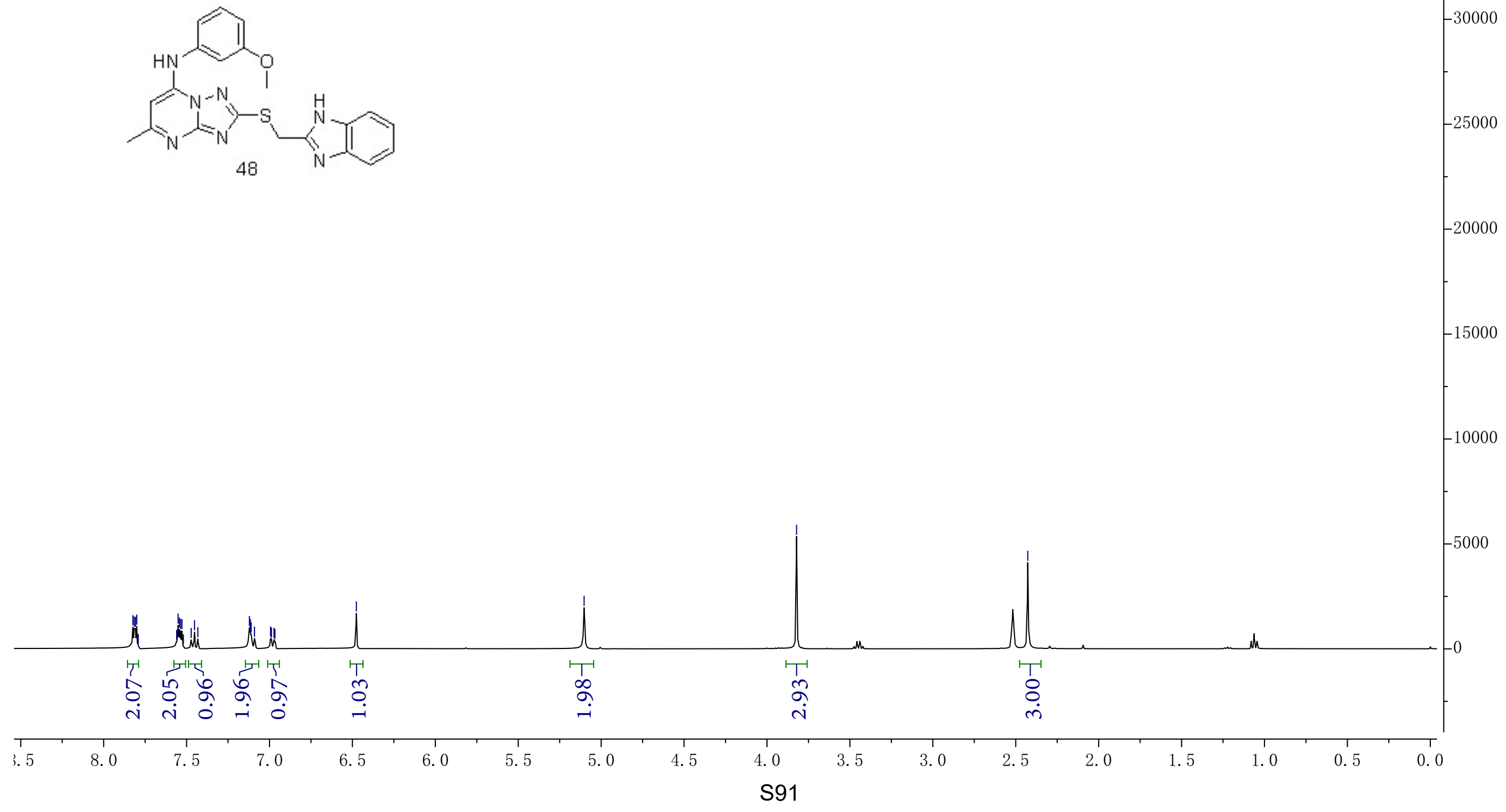

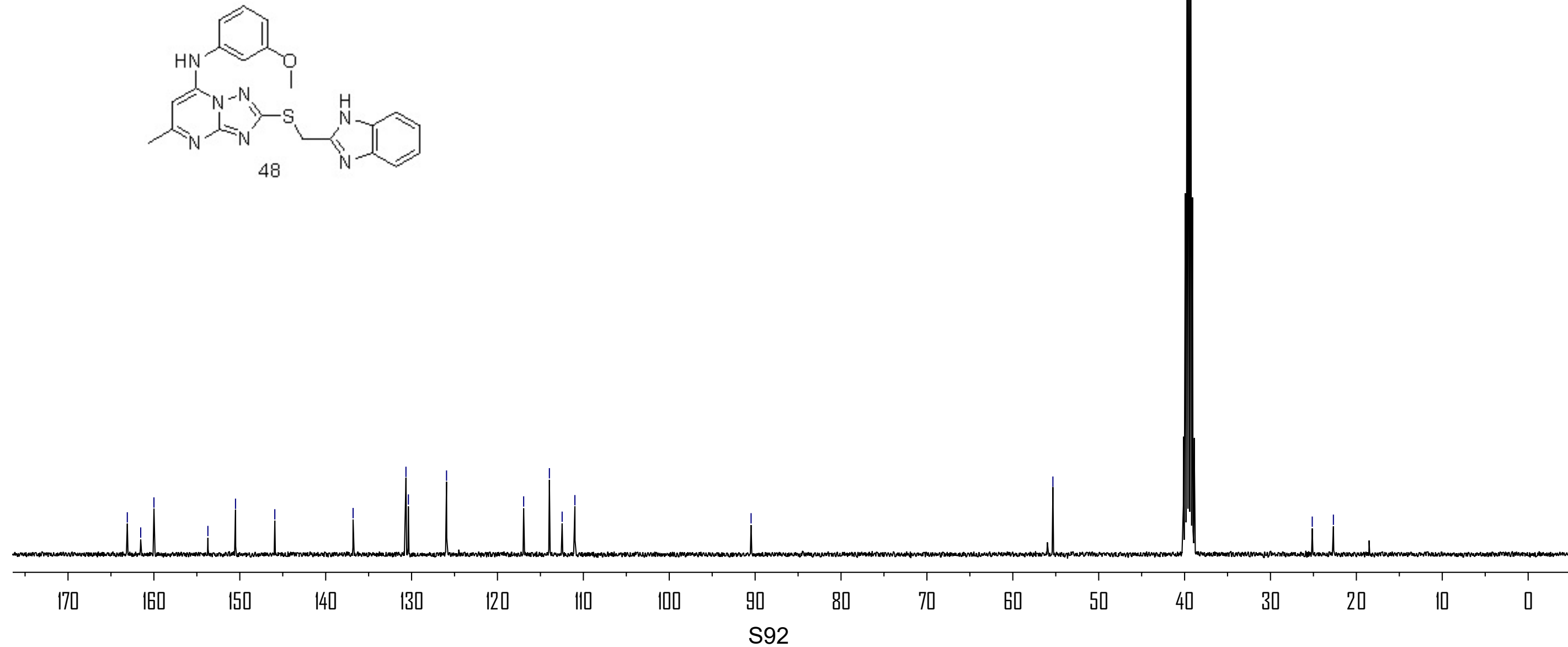


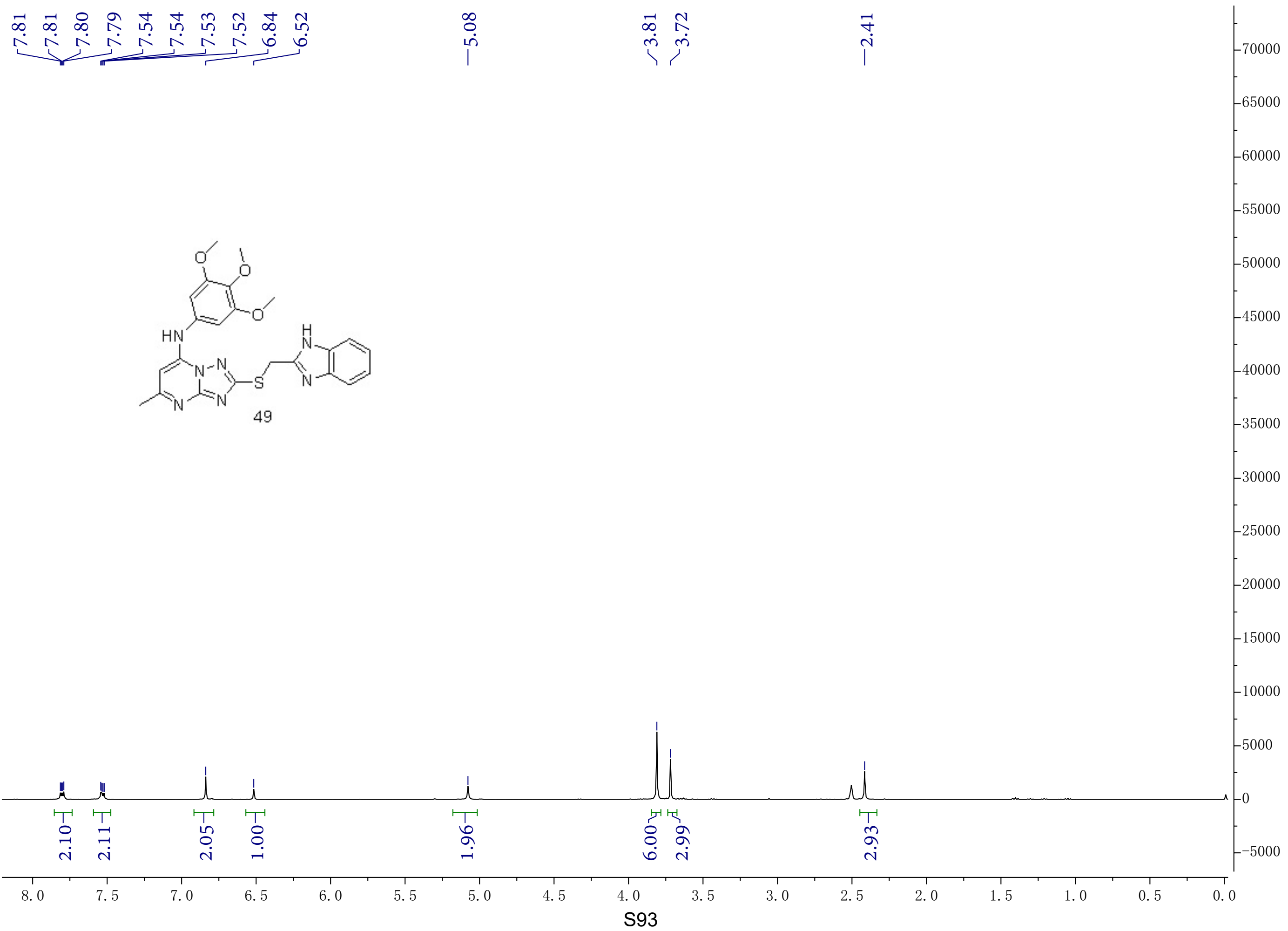



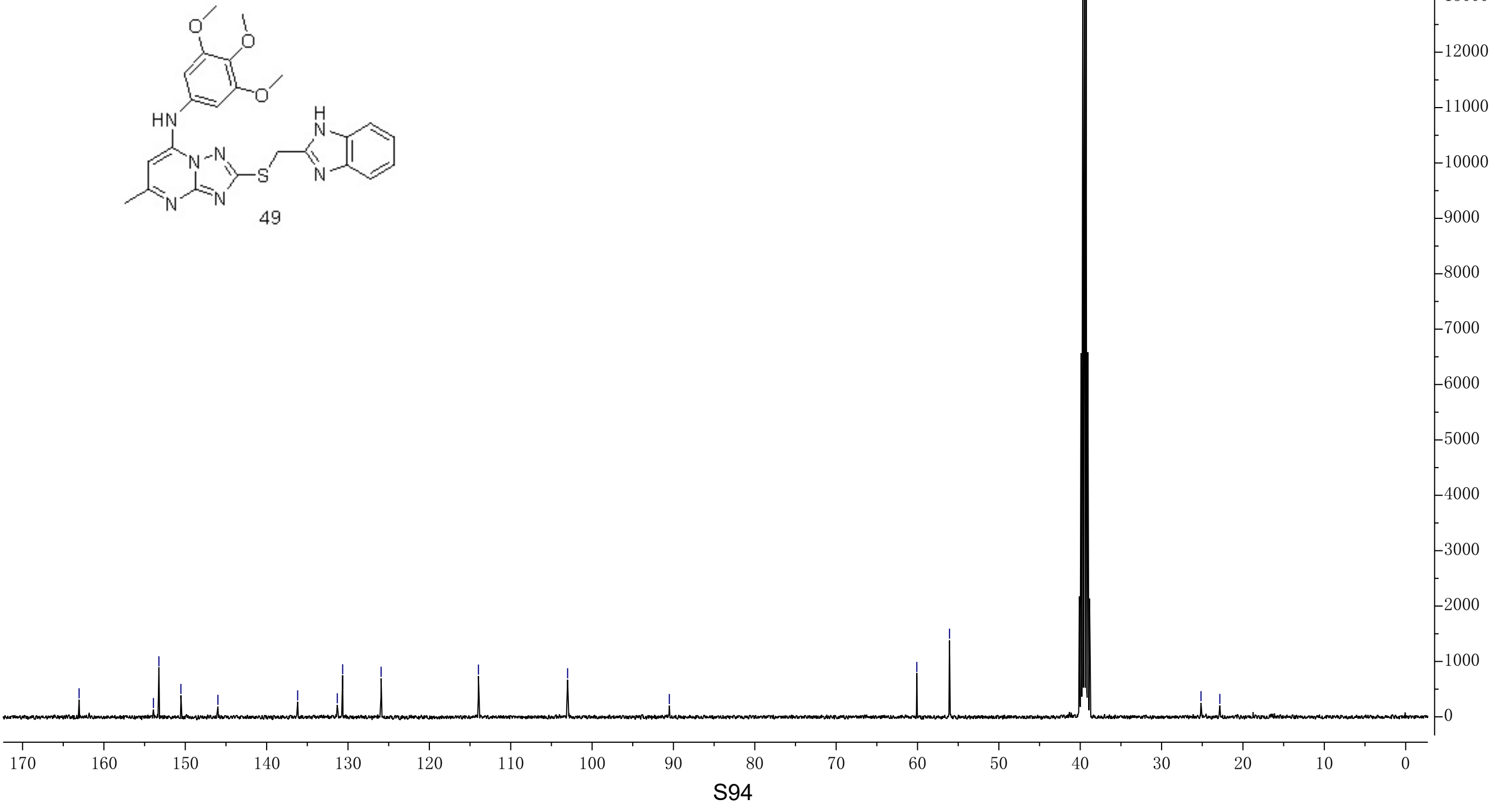

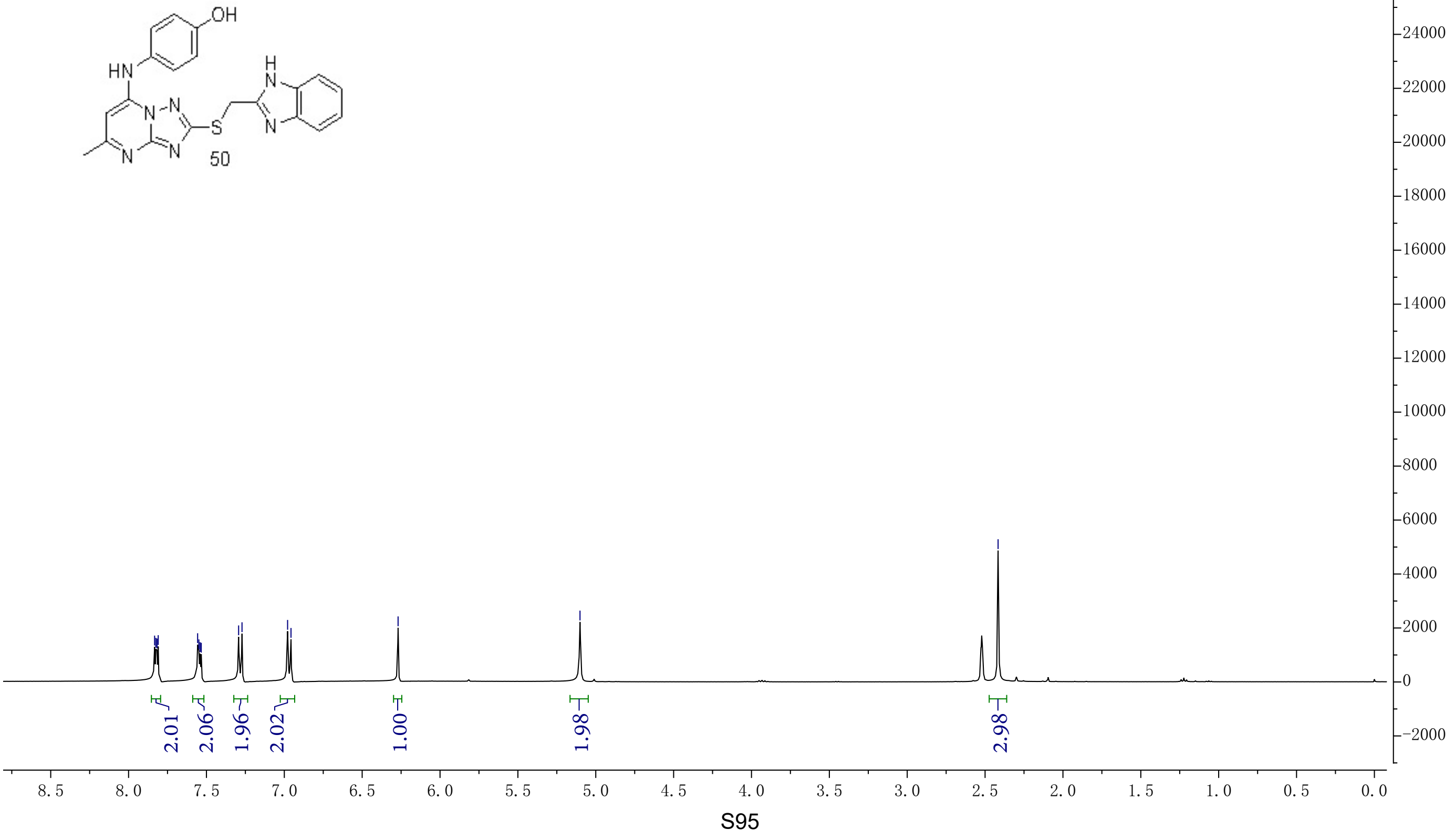
ㄴํํㄷำ

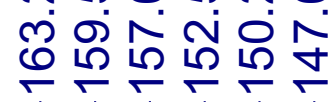

늄용

$\stackrel{\infty}{\circ}$

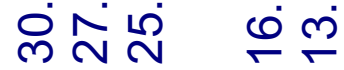

$\bar{m}$

ऑ尓尔

Nㅠ닫

๑

둥

ผึ่

l
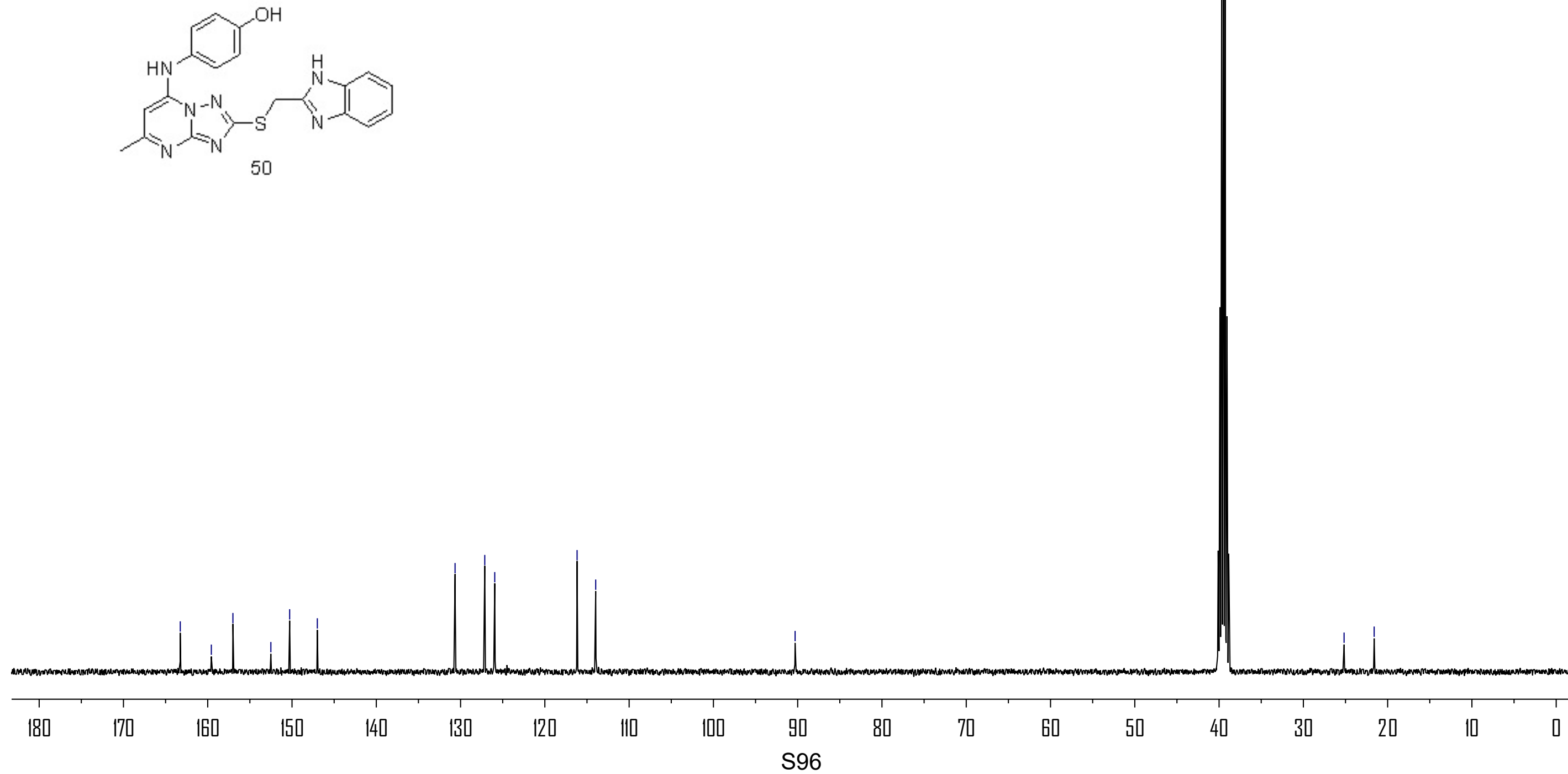

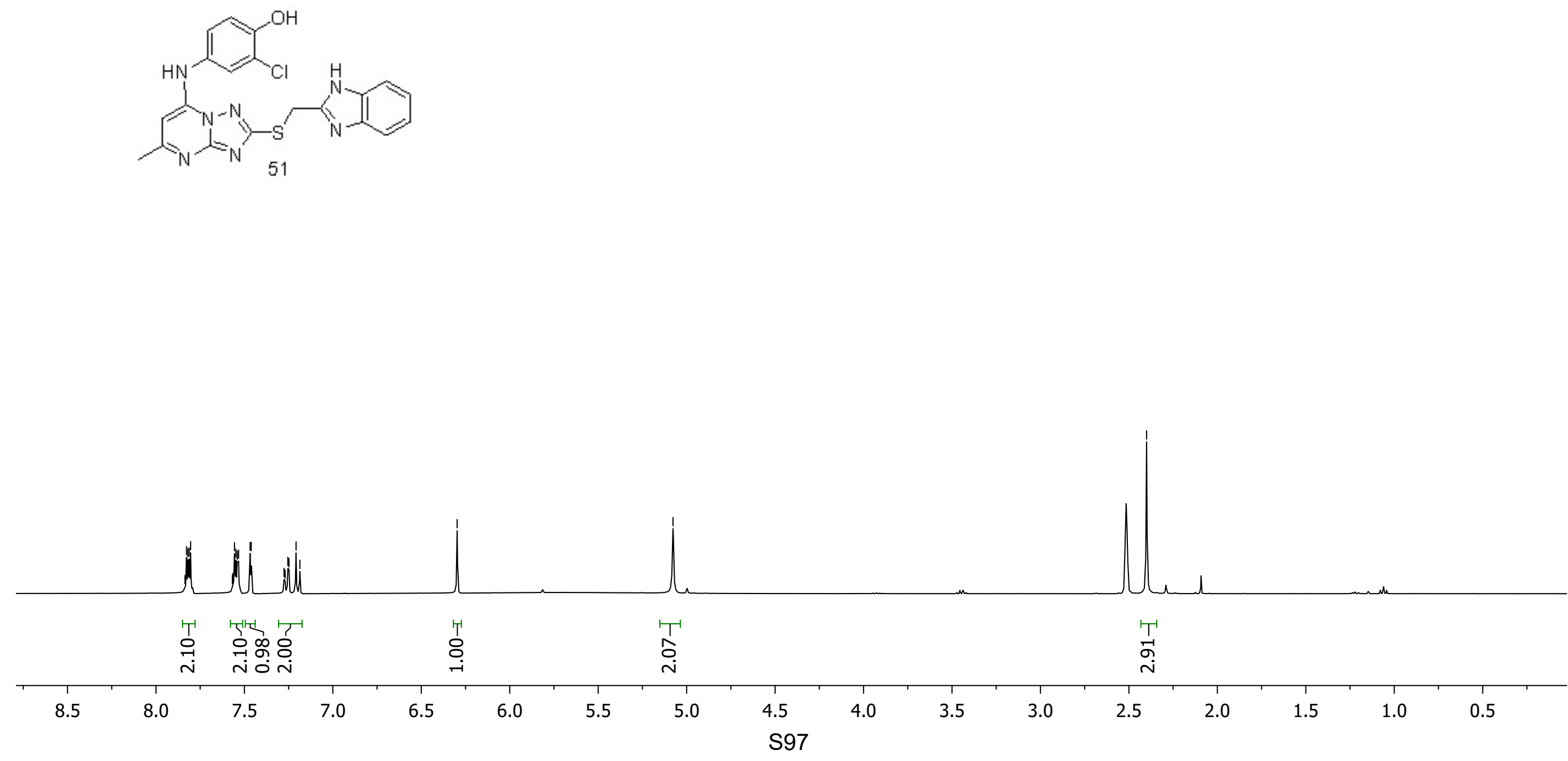


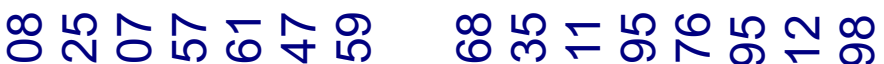

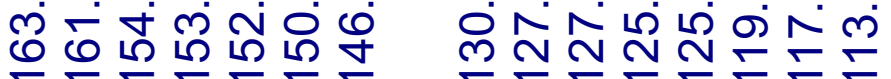

ก

雨
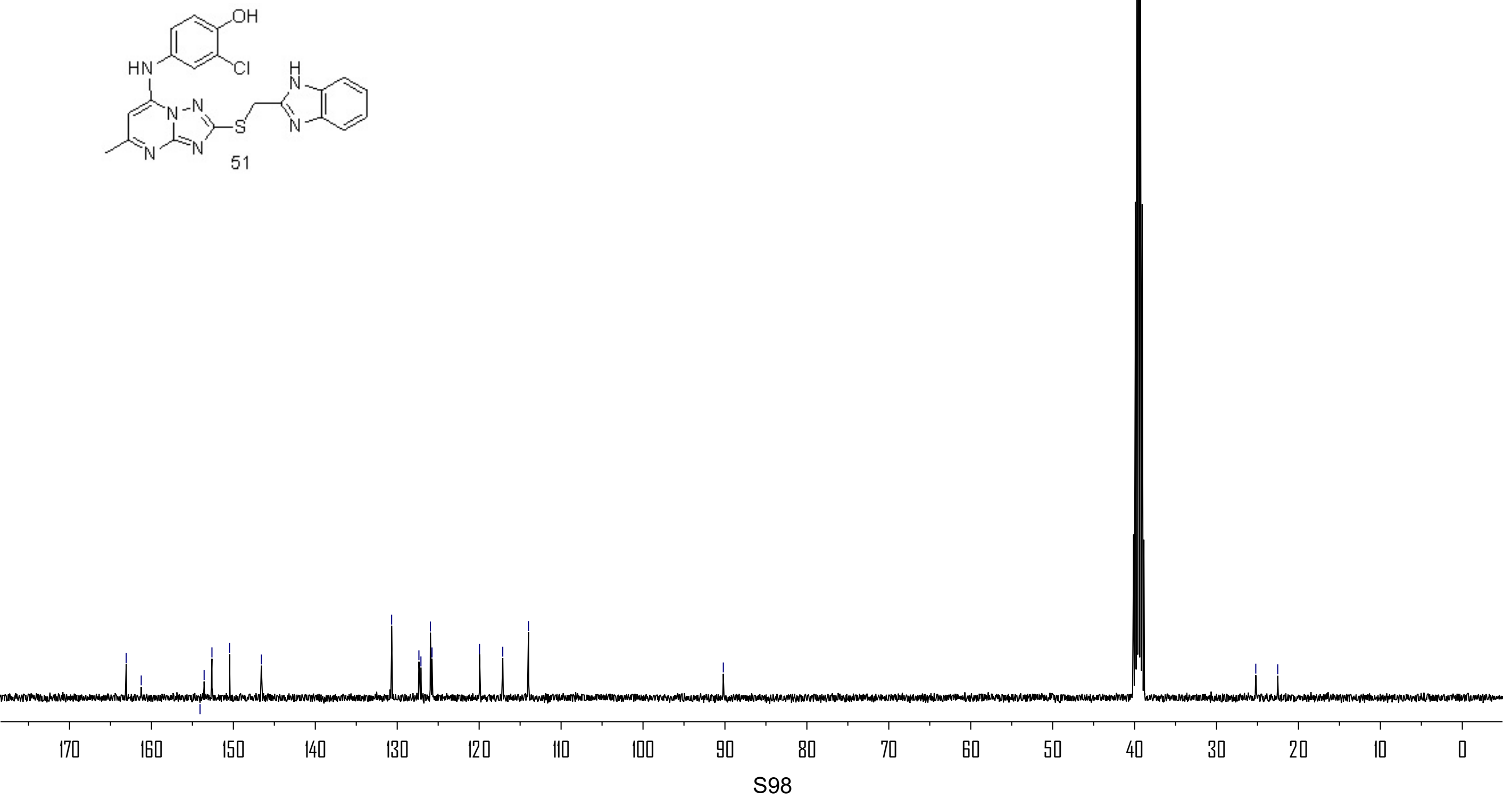

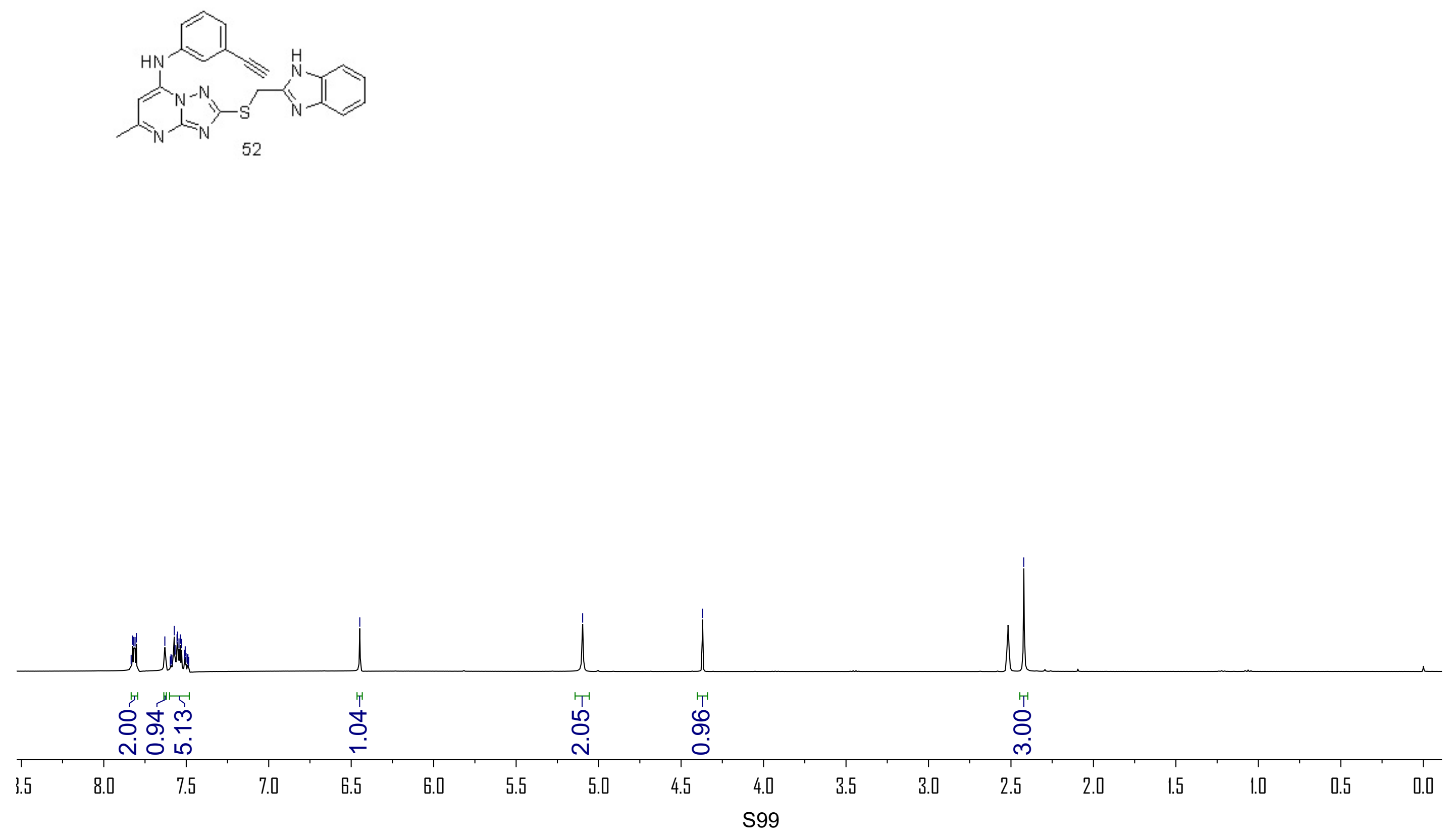


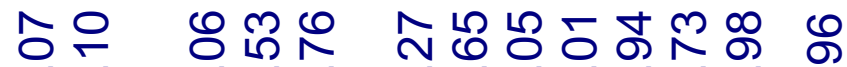

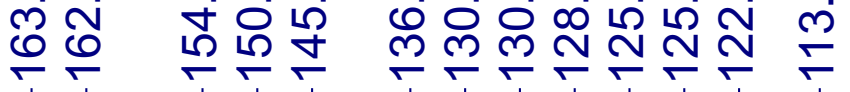

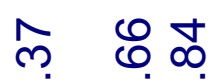

ठํ
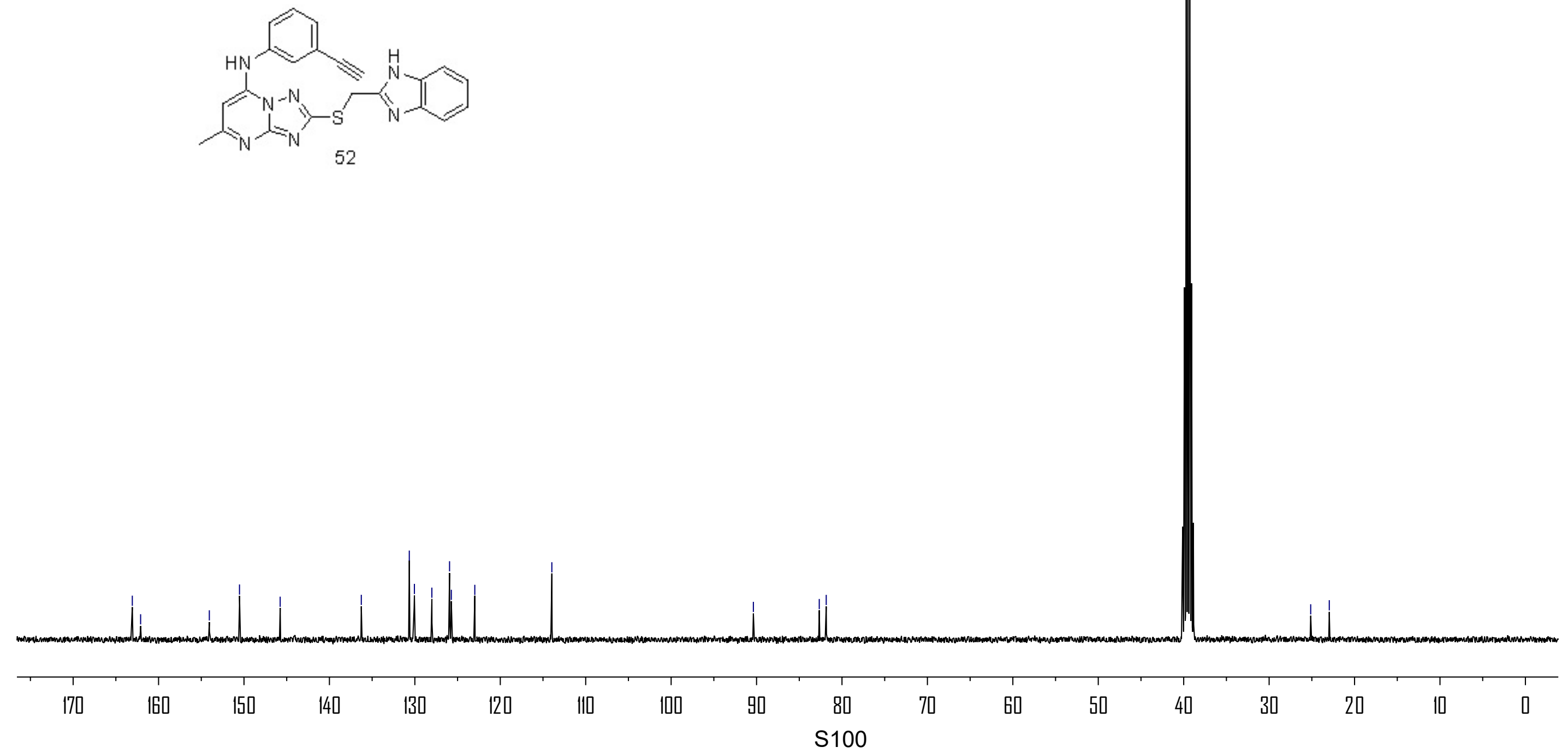

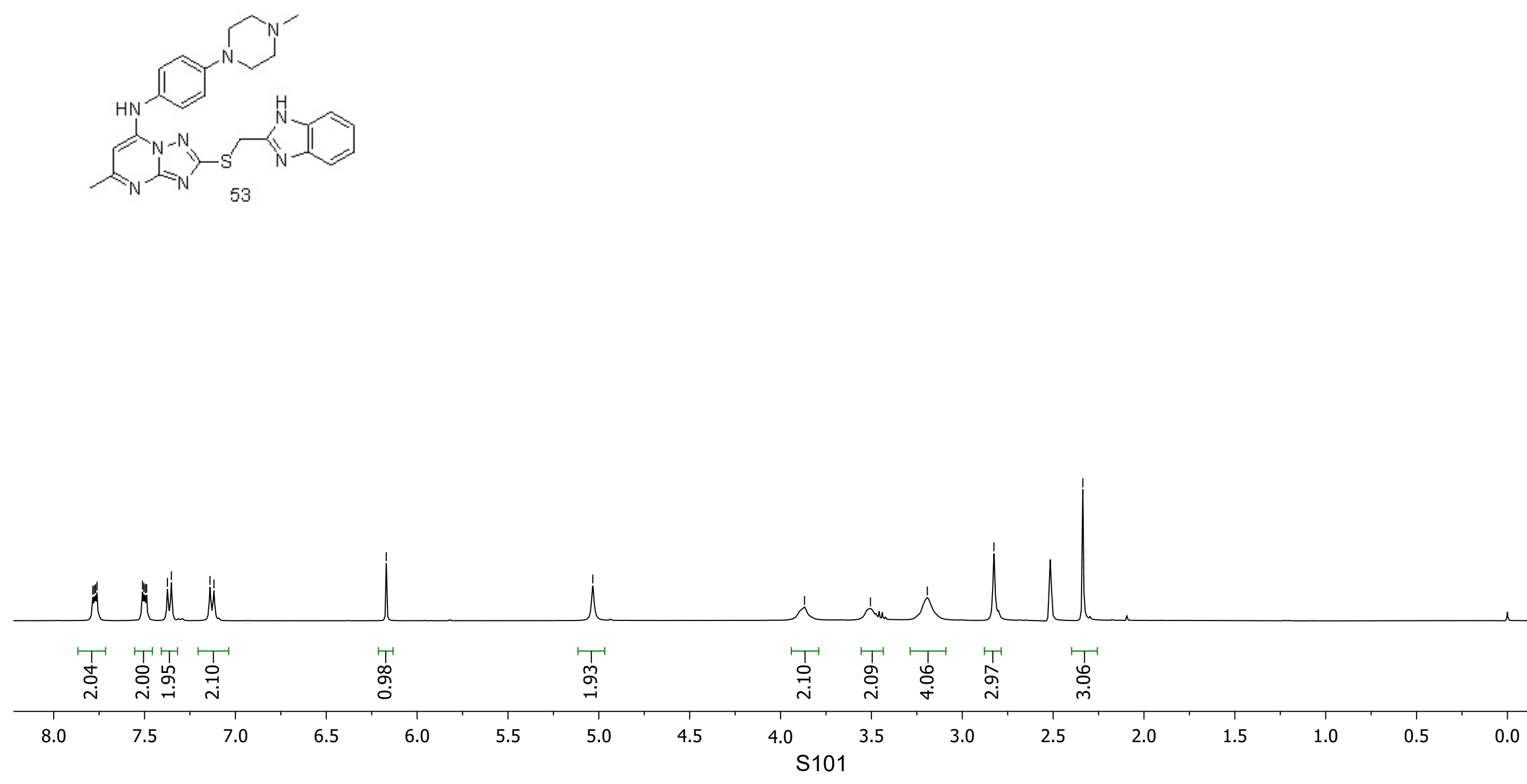


$$
4
$$



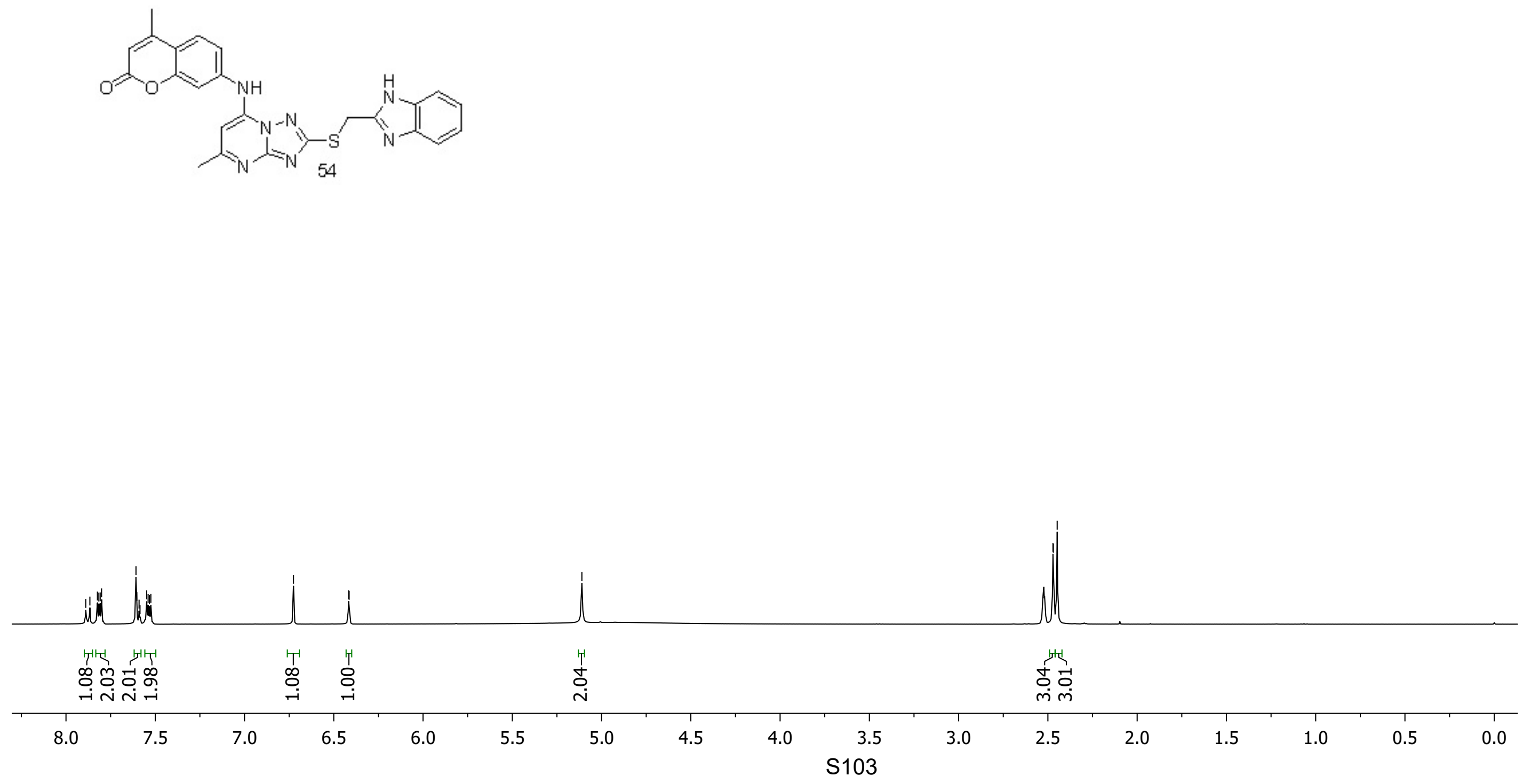

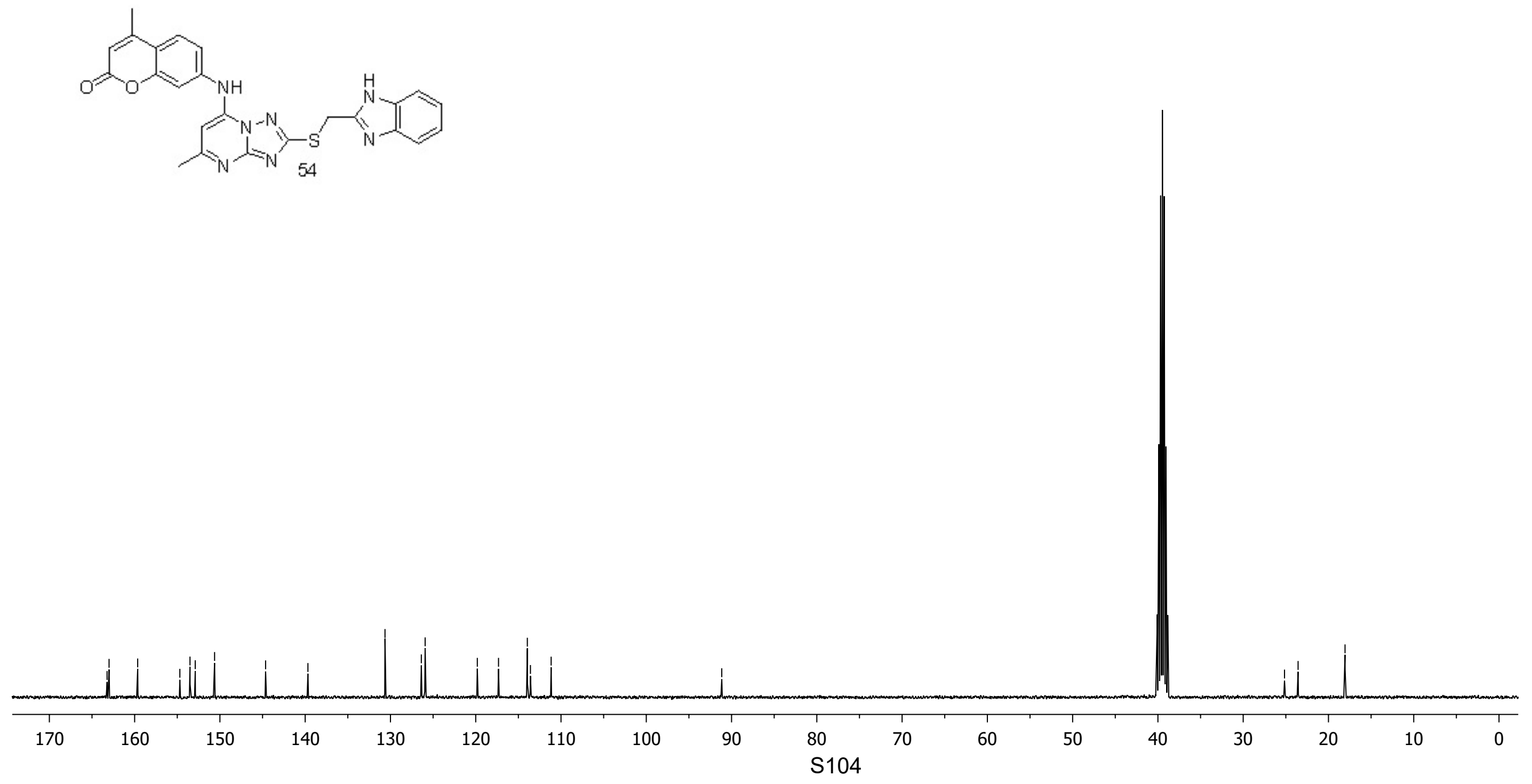

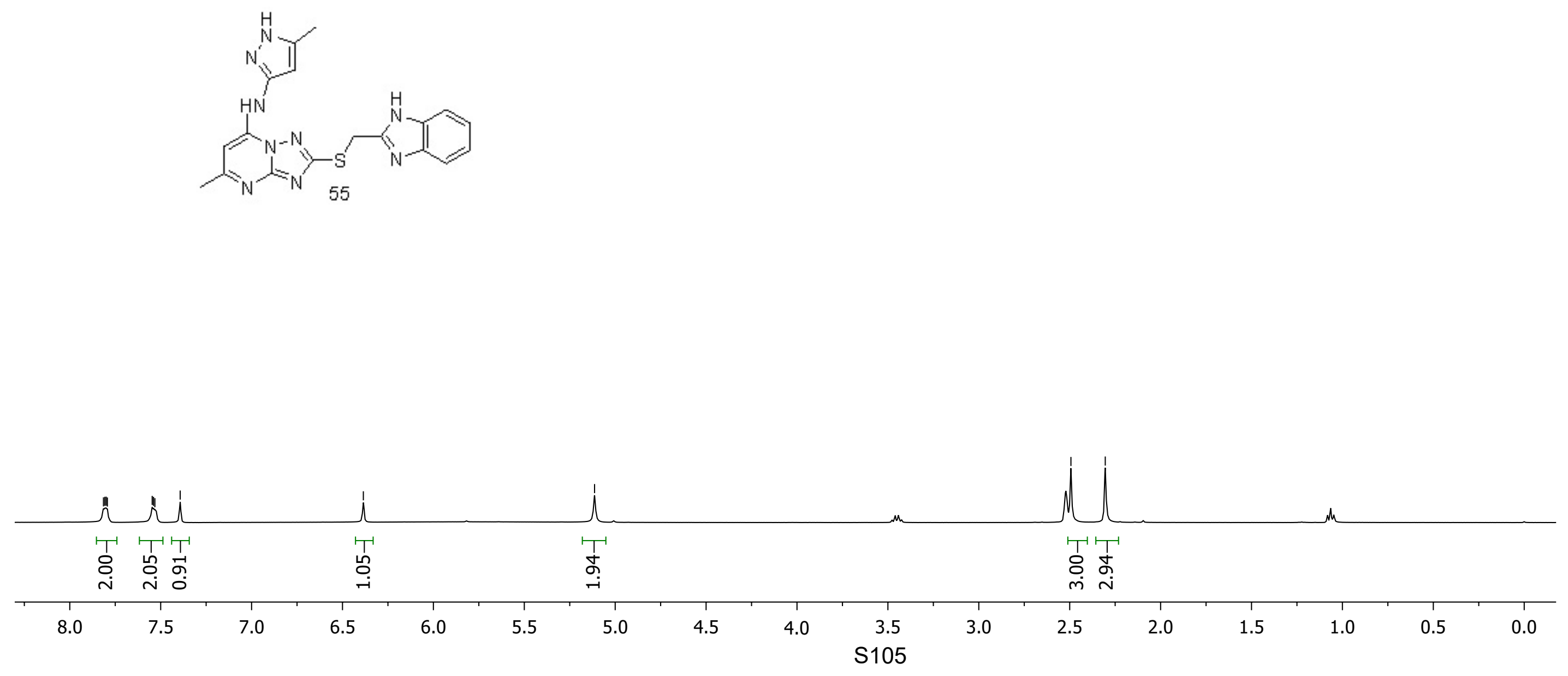

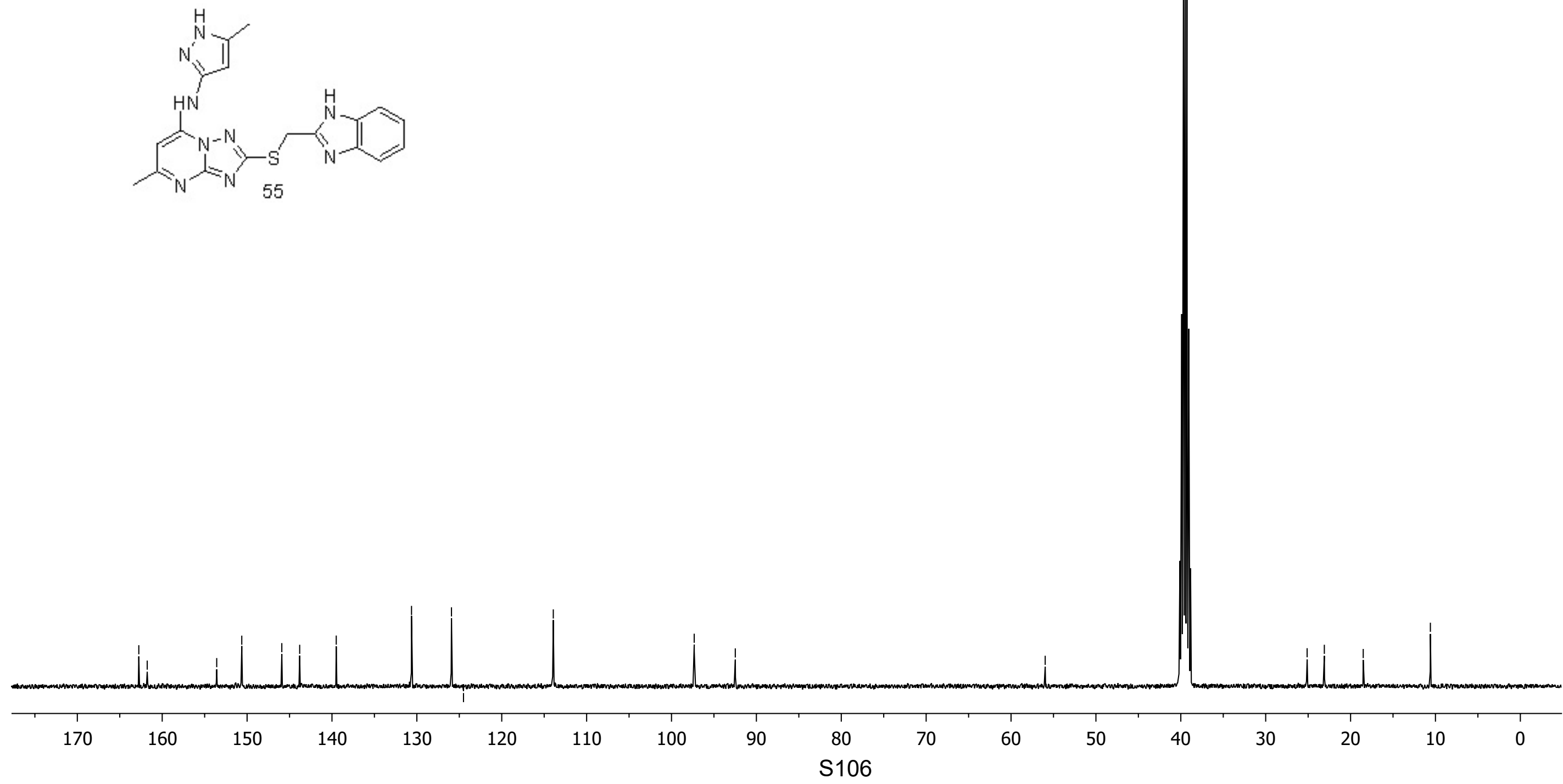
HRMS (ESI): $\mathrm{m} / \mathrm{z}$ calcd for $\mathrm{C}_{12} \mathrm{H}_{11} \mathrm{FN}_{5}(\mathrm{M}+\mathrm{H})^{+}, 244.0998$; found, 244.0997.

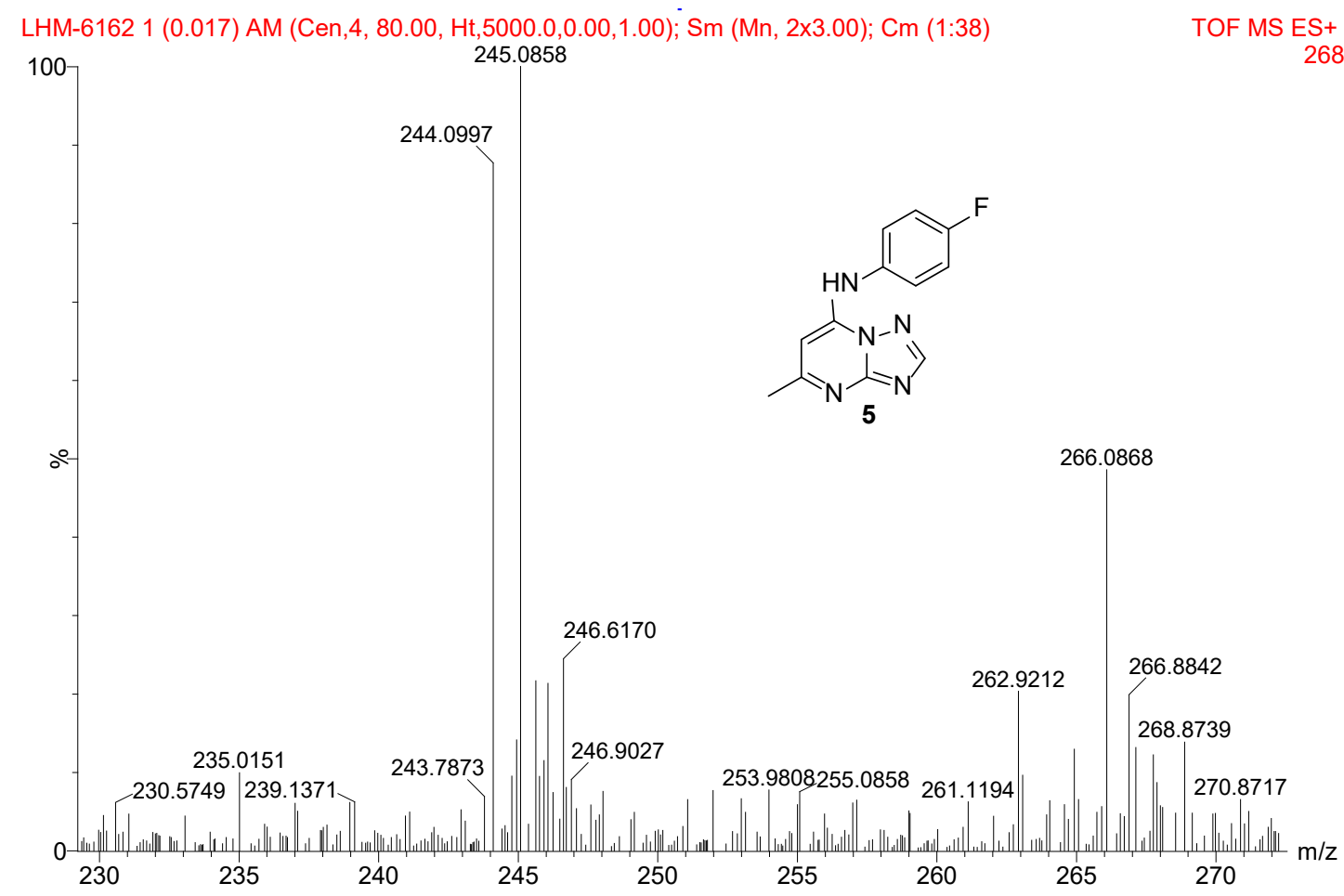

HRMS (ESI): m/z calcd for $\mathrm{C}_{19} \mathrm{H}_{15} \mathrm{FN}_{5} \mathrm{~S}(\mathrm{M}-\mathrm{H})^{+}, 364.1032$; found, 364.1040.

WS-18-NEG\#113 RT: $0.50 \quad$ AV: 1 NL: 2.21 E8

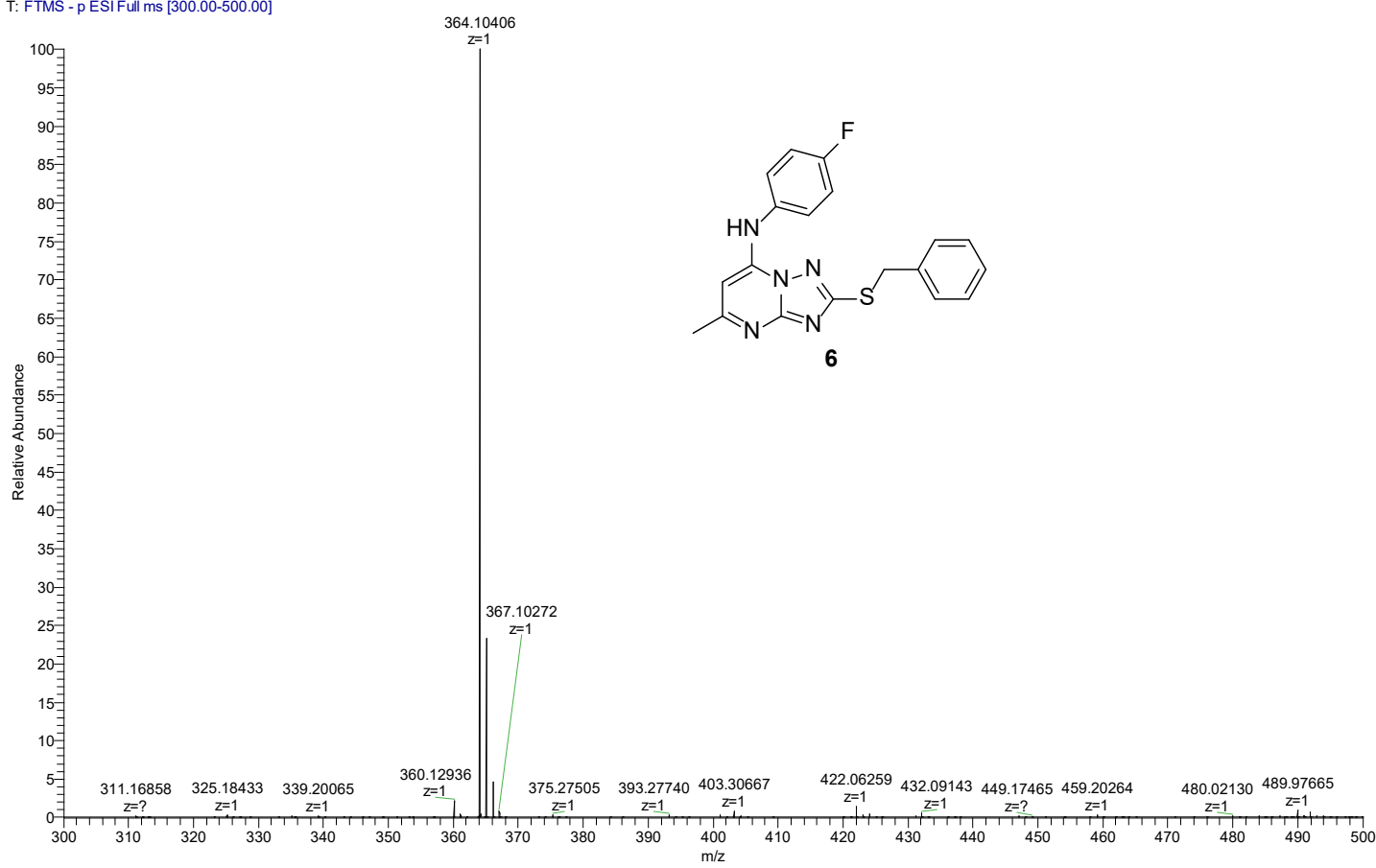


HRMS (ESI): $\mathrm{m} / \mathrm{z}$ calcd for $\mathrm{C}_{20} \mathrm{H}_{19} \mathrm{FN}_{5} \mathrm{~S}(\mathrm{M}+\mathrm{H})^{+}, 380.1345$; found, 380.1331 .

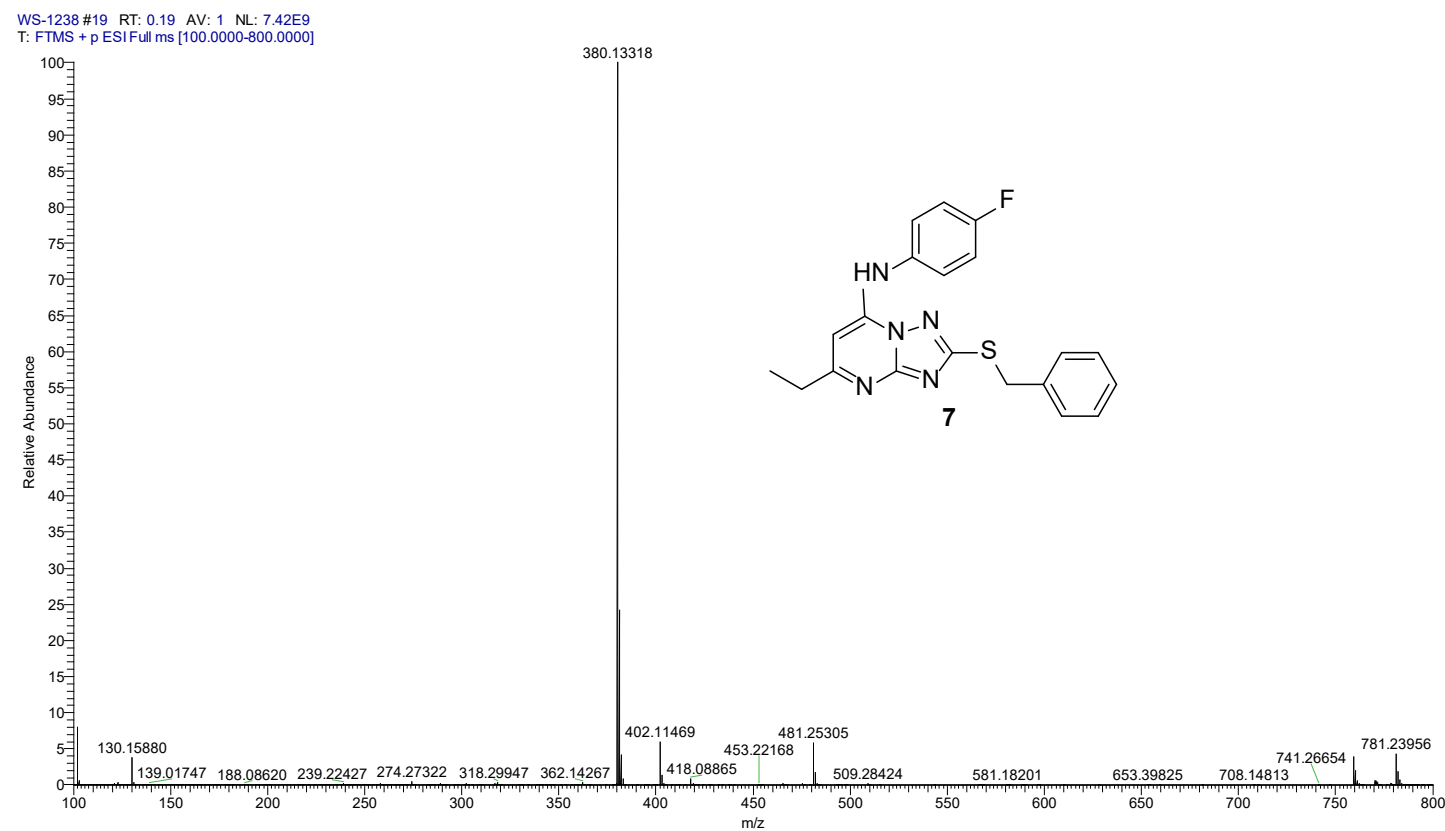

HRMS (ESI): $\mathrm{m} / \mathrm{z}$ calcd for $\mathrm{C}_{21} \mathrm{H}_{21} \mathrm{FN}_{5} \mathrm{~S}(\mathrm{M}+\mathrm{H})^{+}, 394.1502$; found, 394.1487.

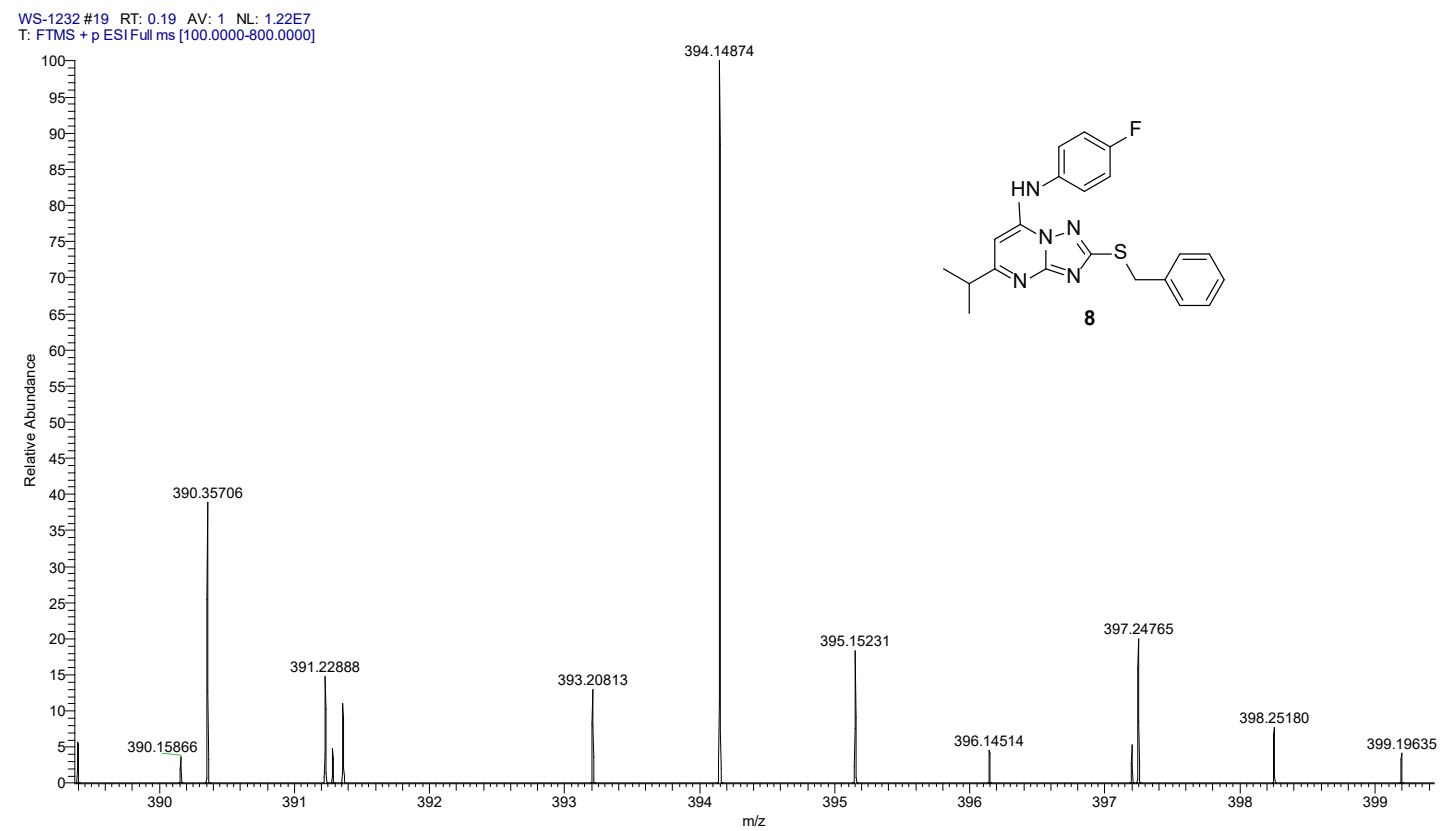


HRMS (ESI): $\mathrm{m} / \mathrm{z}$ calcd for $\mathrm{C}_{19} \mathrm{H}_{16} \mathrm{~F}_{2} \mathrm{~N}_{5} \mathrm{~S}(\mathrm{M}+\mathrm{H})^{+}, 384.1094$; found, 384.1093.

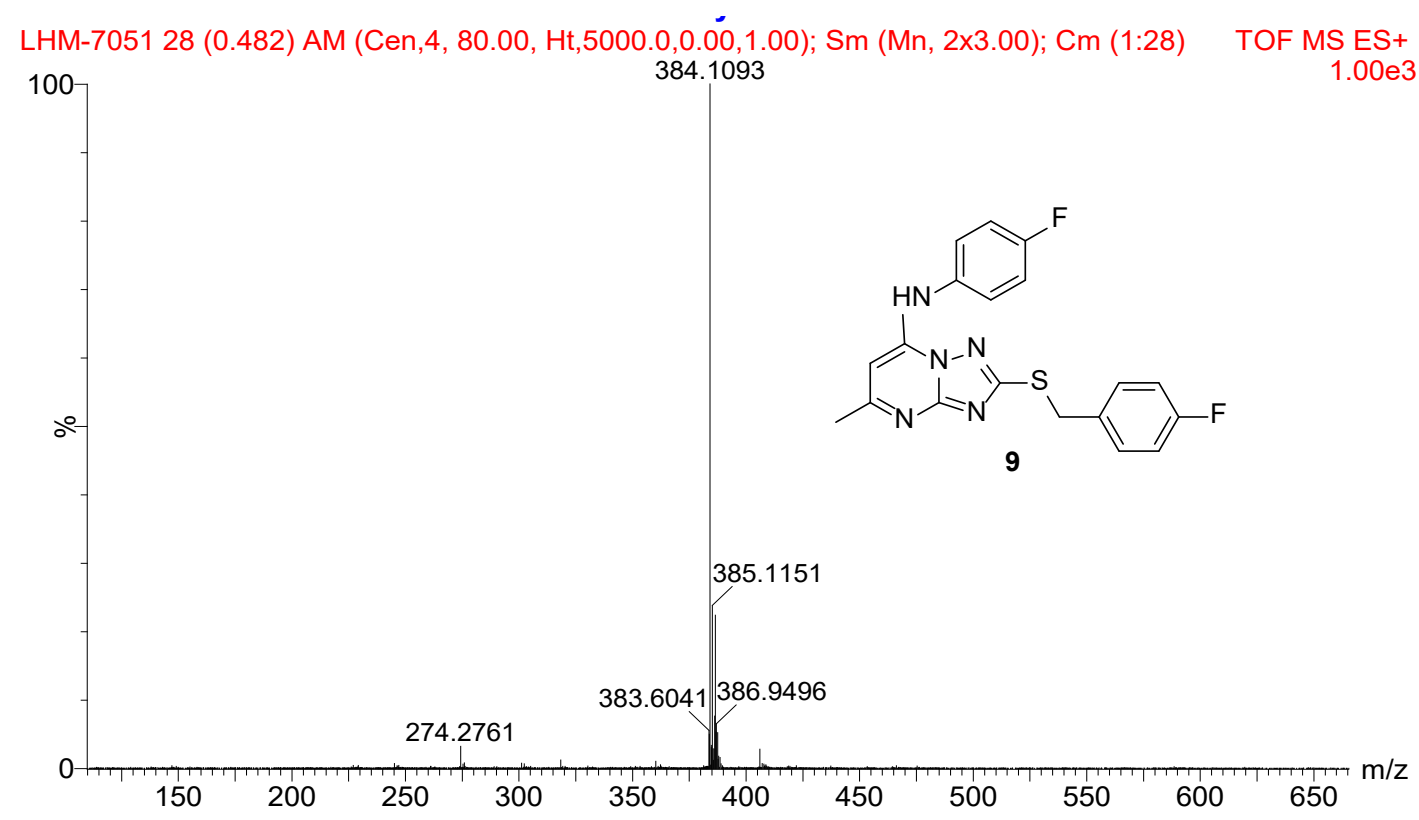

HRMS (ESI): m/z calcd for $\mathrm{C}_{19} \mathrm{H}_{16} \mathrm{ClFN}_{5} \mathrm{~S}(\mathrm{M}+\mathrm{H})^{+}$, 400.0799; found, 400.0799 .

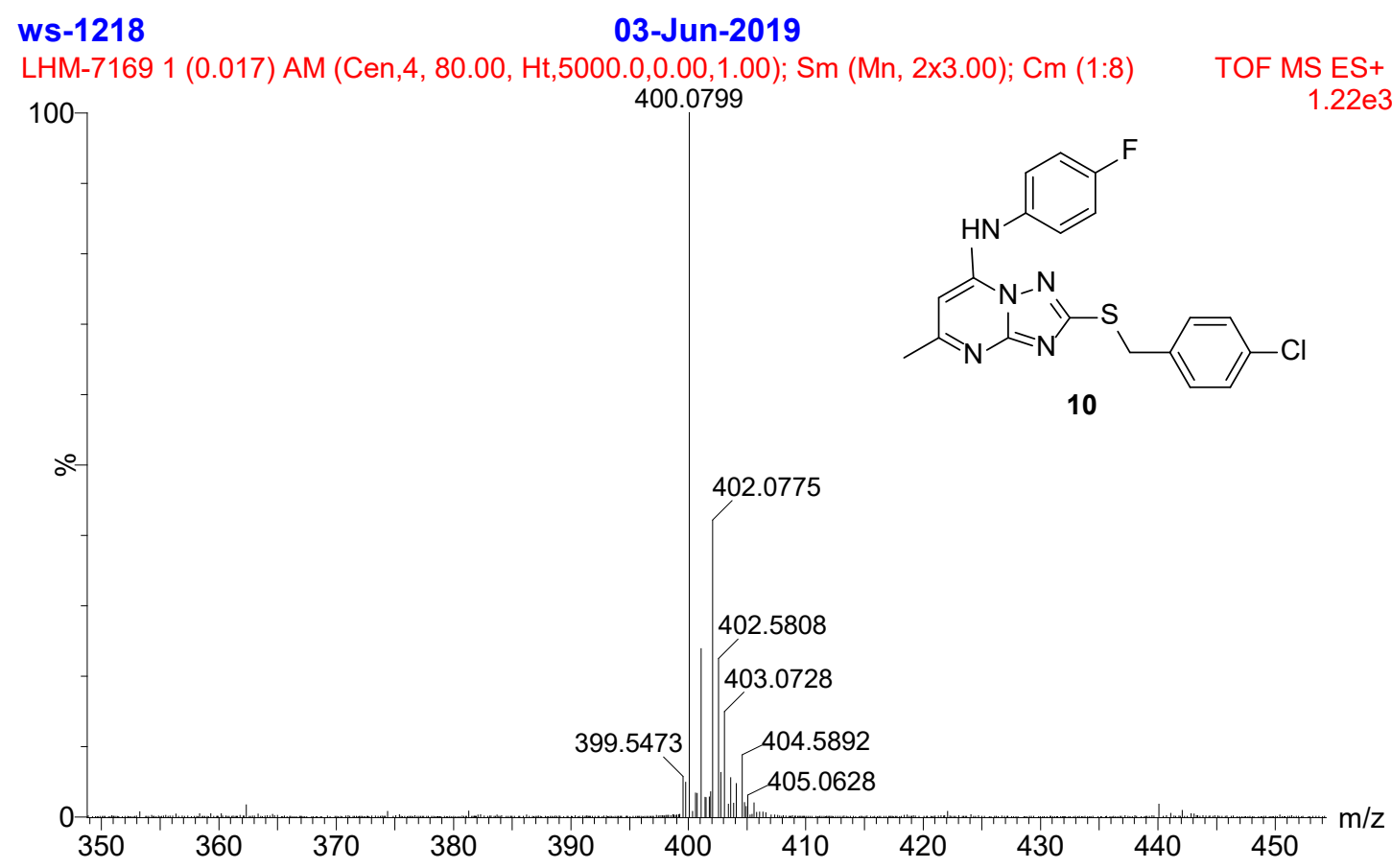


HRMS (ESI): m/z calcd for $\mathrm{C}_{19} \mathrm{H}_{16} \mathrm{BrFN}_{5} \mathrm{~S}(\mathrm{M}+\mathrm{H})^{+}$, 444.0294; found,444.0276.

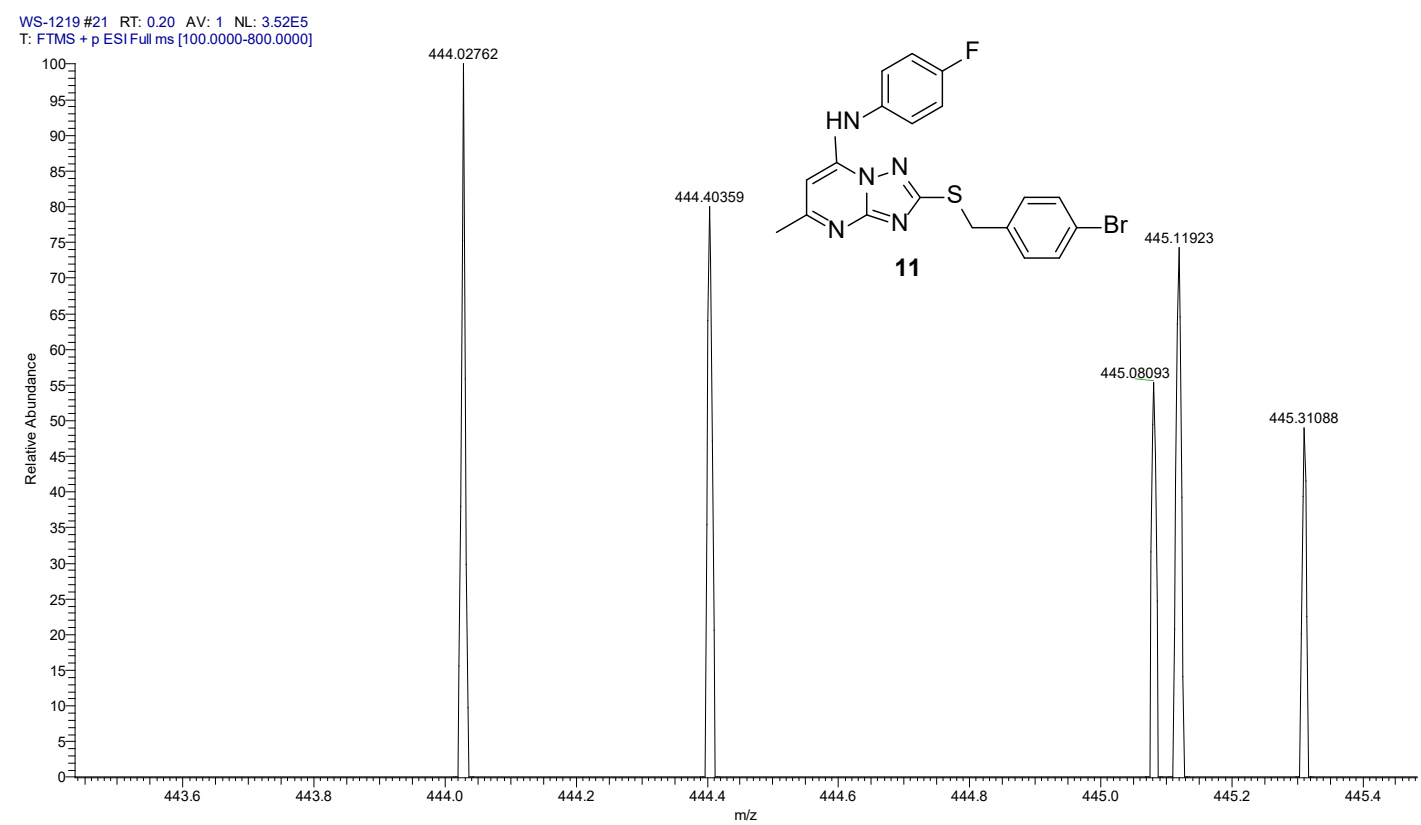

HRMS (ESI): m/z calcd for $\mathrm{C}_{19} \mathrm{H}_{16} \mathrm{BrFN}_{5} \mathrm{~S}(\mathrm{M}+\mathrm{H})^{+}, 444.0294$; found,444.0281.

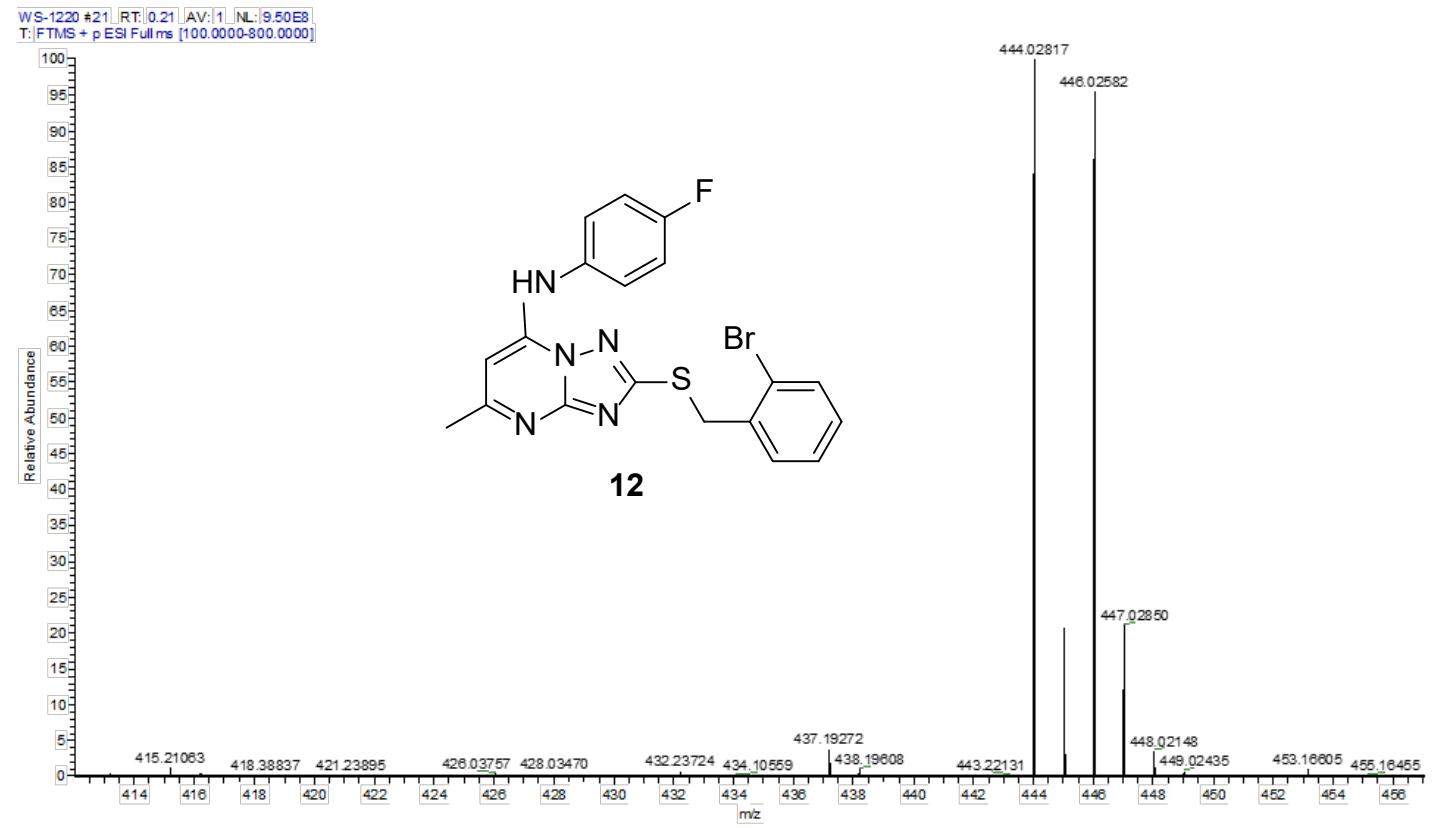


HRMS (ESI): m/z calcd for $\mathrm{C}_{19} \mathrm{H}_{16} \mathrm{BrFN}_{5} \mathrm{~S}(\mathrm{M}+\mathrm{H})^{+}$, 444.0294; found,444.0281.

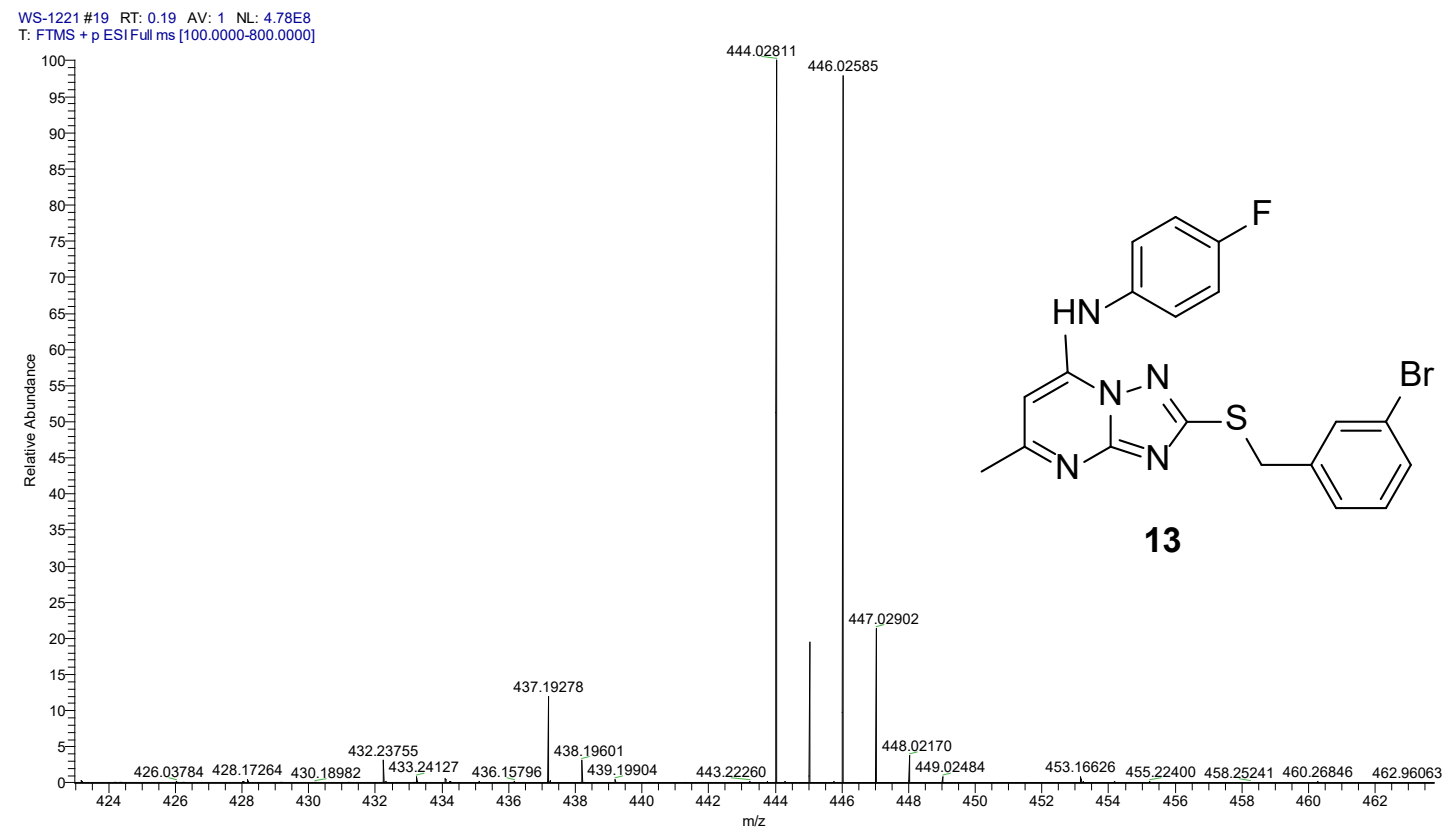

HRMS (ESI): $\mathrm{m} / \mathrm{z}$ calcd for $\mathrm{C}_{20} \mathrm{H}_{16} \mathrm{~F}_{4} \mathrm{~N}_{5} \mathrm{~S}(\mathrm{M}+\mathrm{H})^{+}, 434.1063$; found, 434.1062.

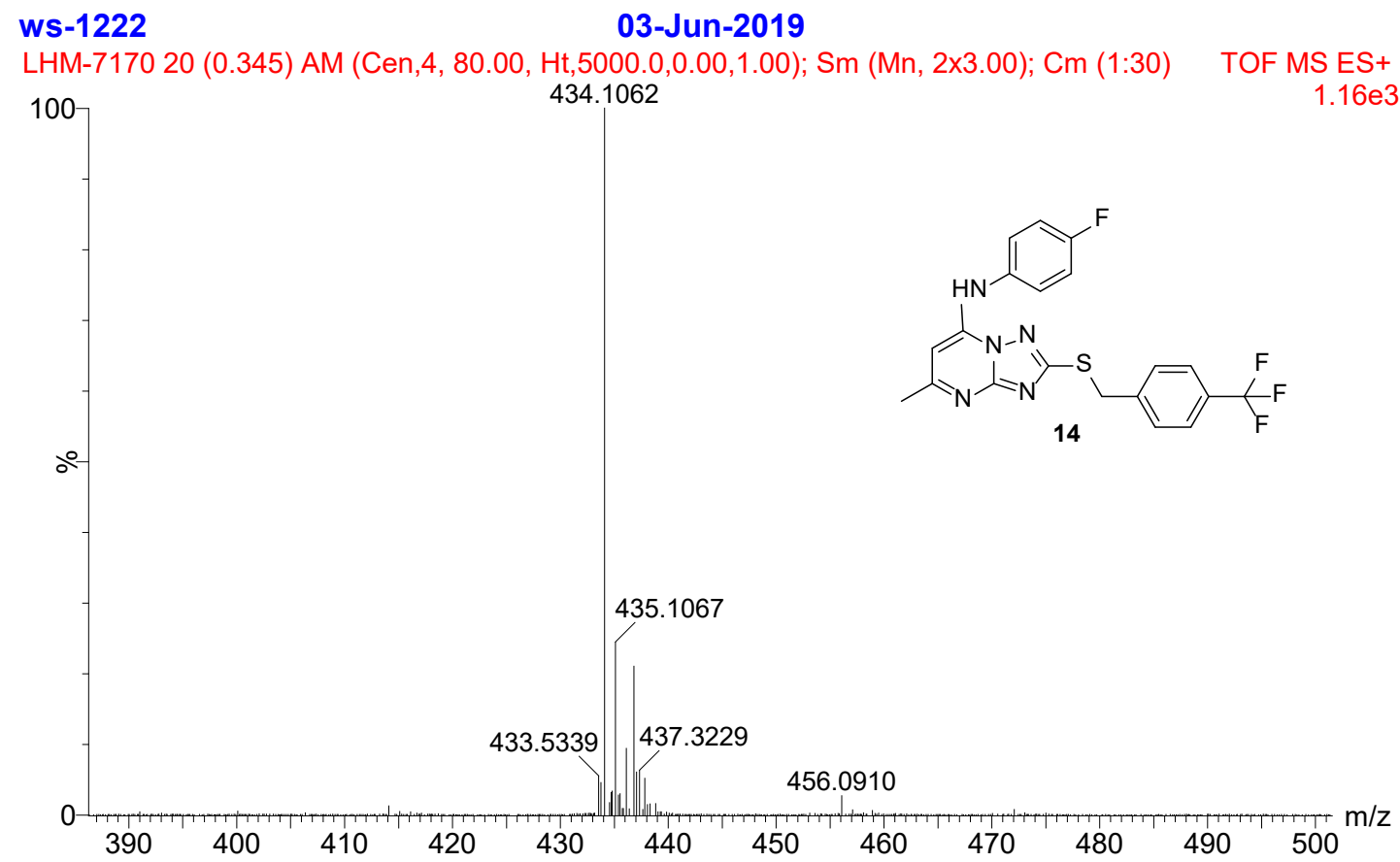


HRMS (ESI): m/z calcd for $\mathrm{C}_{19} \mathrm{H}_{16} \mathrm{FN}_{6} \mathrm{O}_{2} \mathrm{~S}(\mathrm{M}+\mathrm{H})^{+}, 411.1039$; found,411.1024.

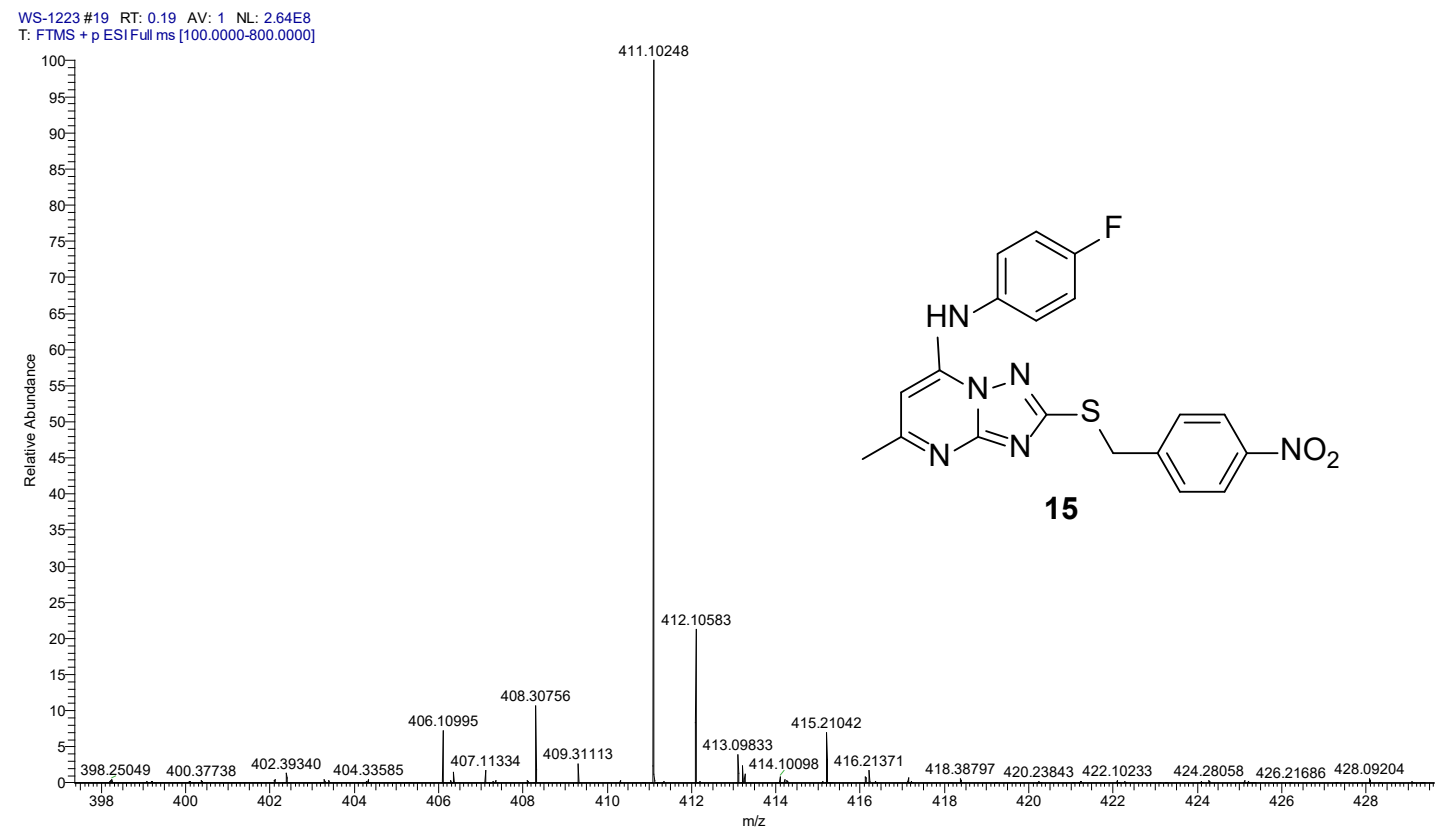

HRMS (ESI): m/z calcd for $\mathrm{C}_{19} \mathrm{H}_{16} \mathrm{FN}_{6} \mathrm{O}_{2} \mathrm{~S}(\mathrm{M}+\mathrm{H})^{+}, 411.1039$; found,411.1024.

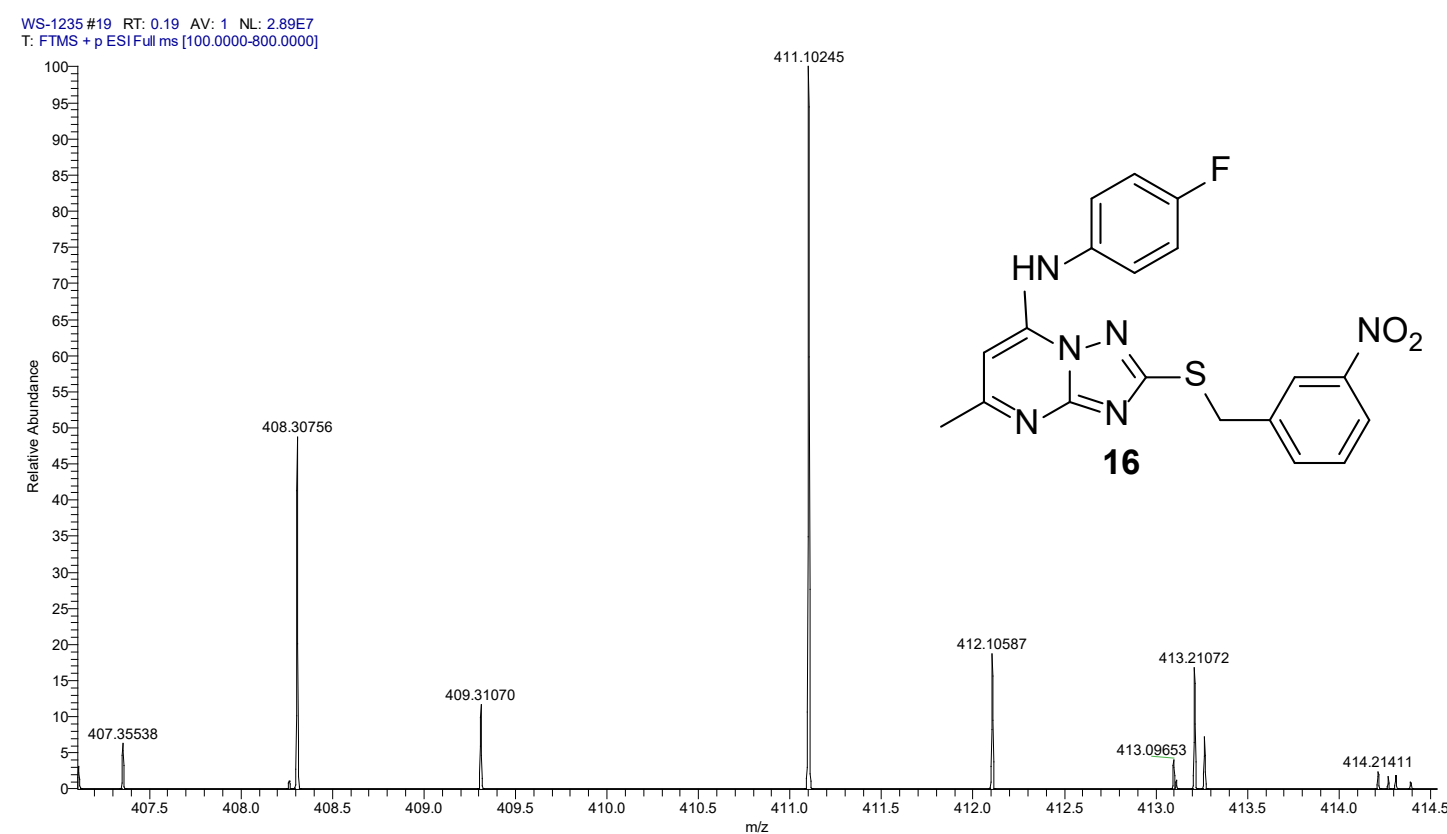


HRMS (ESI): $\mathrm{m} / \mathrm{z}$ calcd for $\mathrm{C}_{19} \mathrm{H}_{15} \mathrm{Cl}_{2} \mathrm{FN}_{5} \mathrm{~S}(\mathrm{M}+\mathrm{H})^{+}$, 434.0409; found,434.0396.

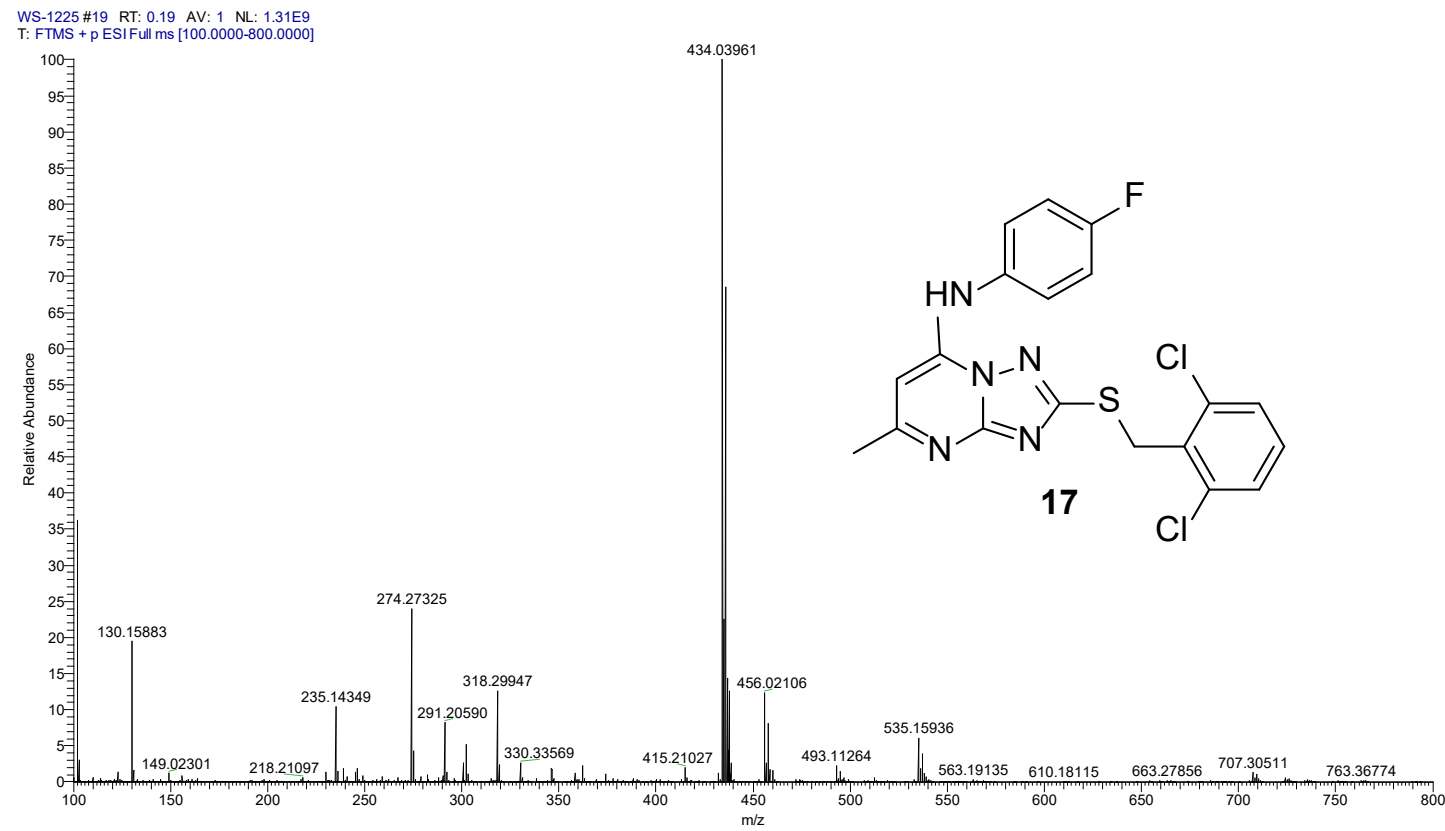

HRMS (ESI): $\mathrm{m} / \mathrm{z}$ calcd for $\mathrm{C}_{20} \mathrm{H}_{19} \mathrm{FN}_{5} \mathrm{~S}(\mathrm{M}+\mathrm{H})^{+}, 380.1345$; found,380.1332.

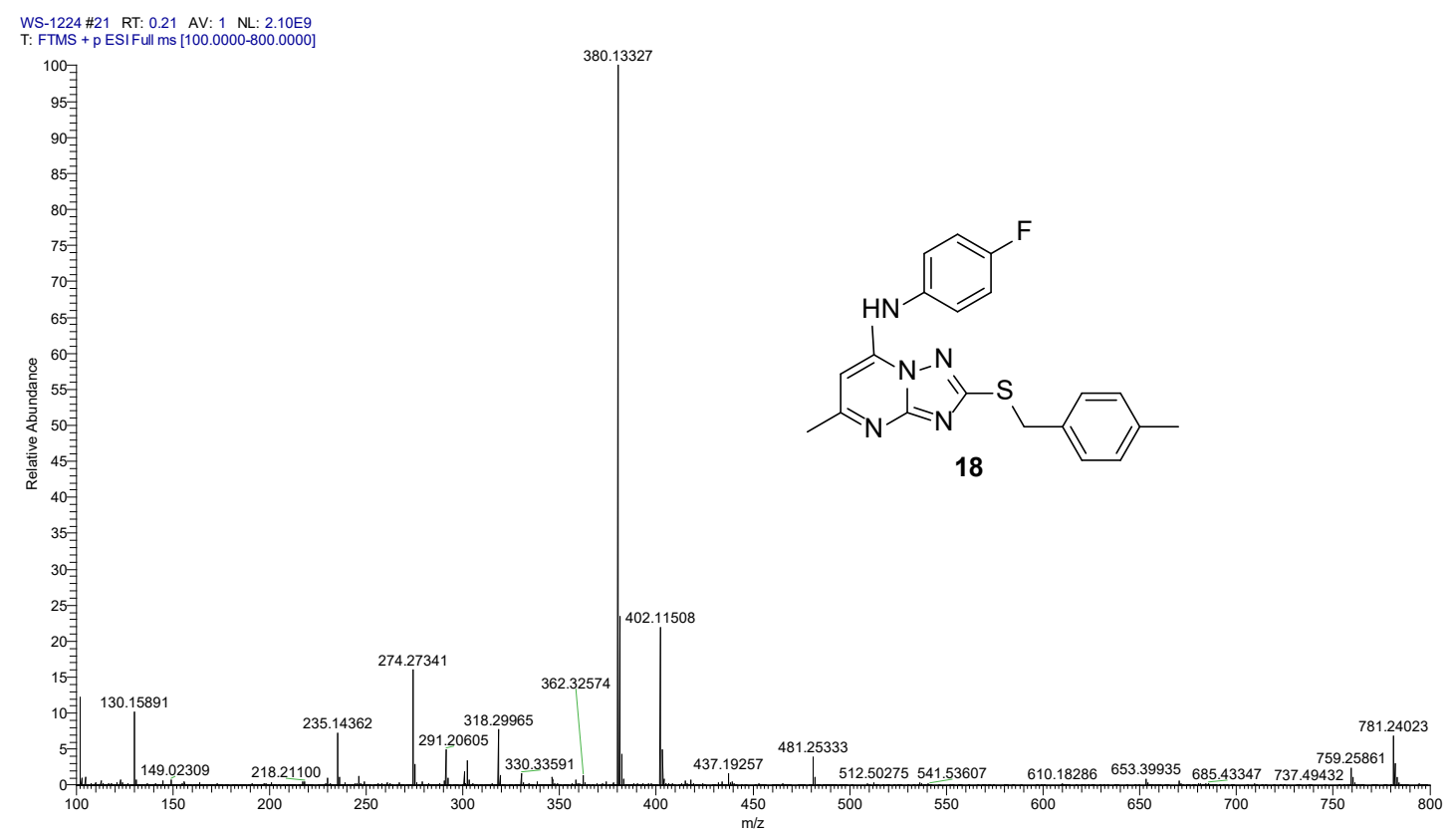


HRMS (ESI): m/z calcd for $\mathrm{C}_{20} \mathrm{H}_{19} \mathrm{FN}_{5} \mathrm{OS}(\mathrm{M}+\mathrm{H})^{+}, 396.1294$; found,396.1280.

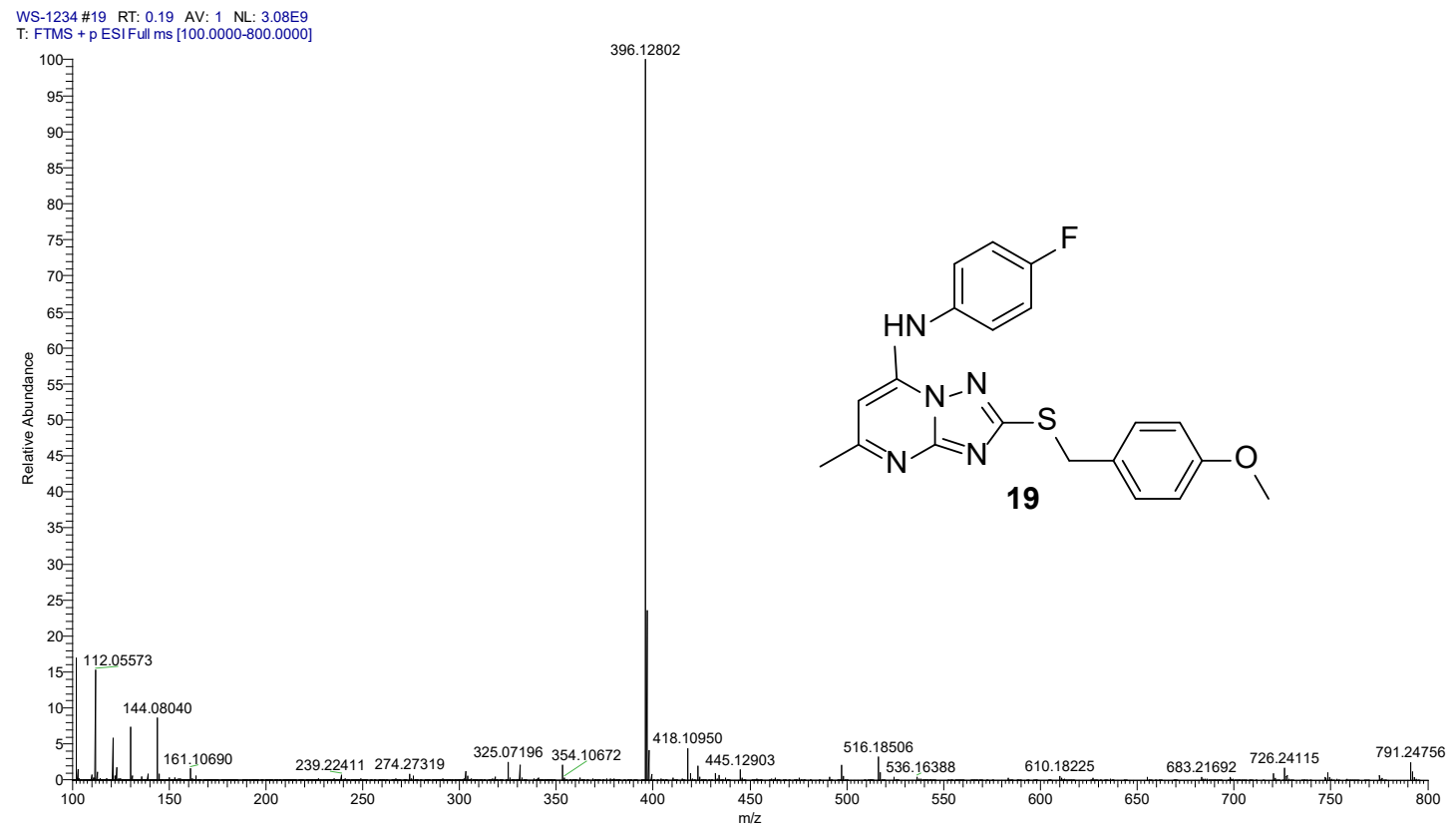

HRMS (ESI): m/z calcd for $\mathrm{C}_{23} \mathrm{H}_{19} \mathrm{FN}_{5} \mathrm{~S}(\mathrm{M}+\mathrm{H})^{+}, 416.1345$; found, 416.1330 .

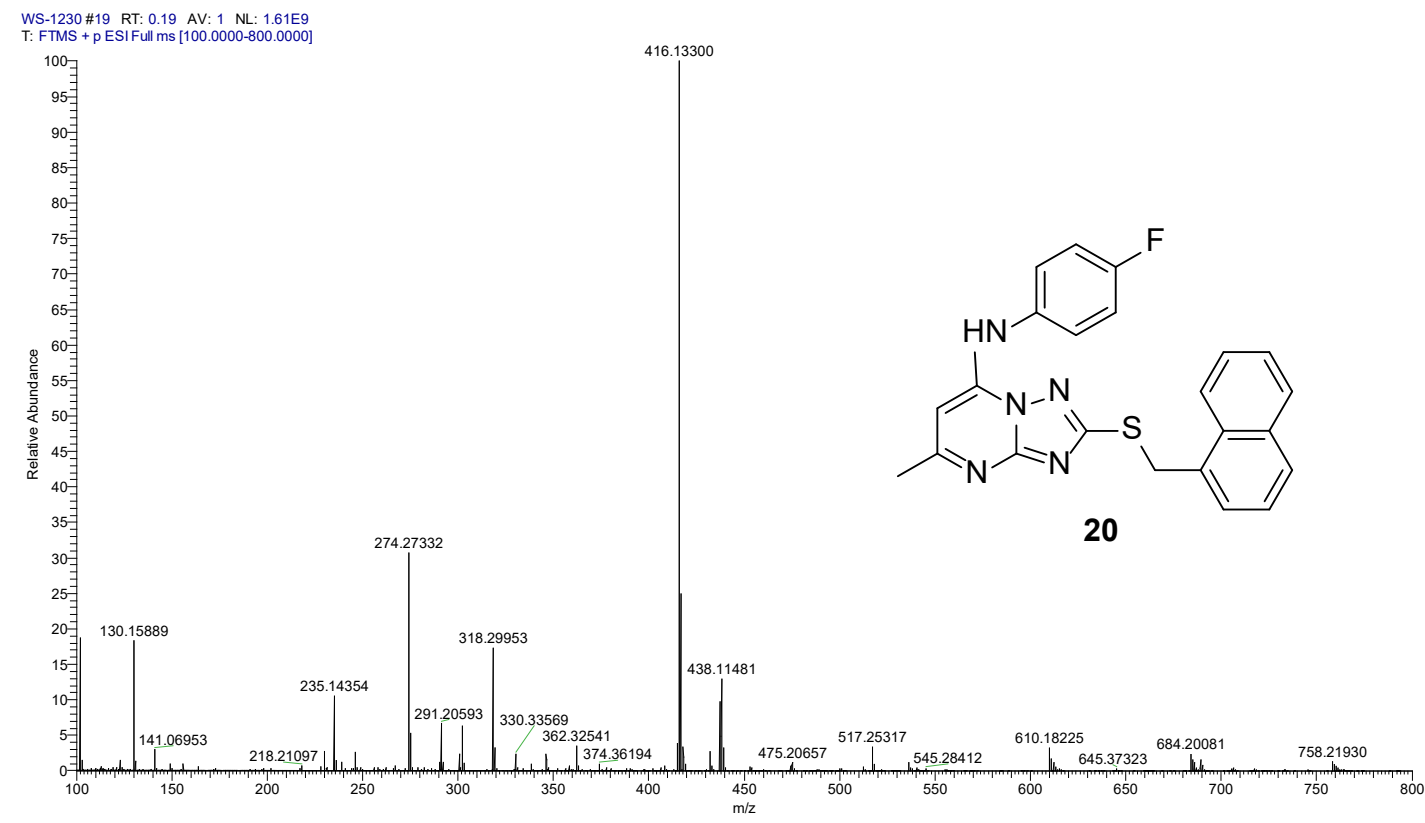


HRMS (ESI): m/z calcd for $\mathrm{C}_{20} \mathrm{H}_{19} \mathrm{FN}_{5} \mathrm{~S}(\mathrm{M}+\mathrm{H})^{+}, 380.1345$; found, 380.1332 .

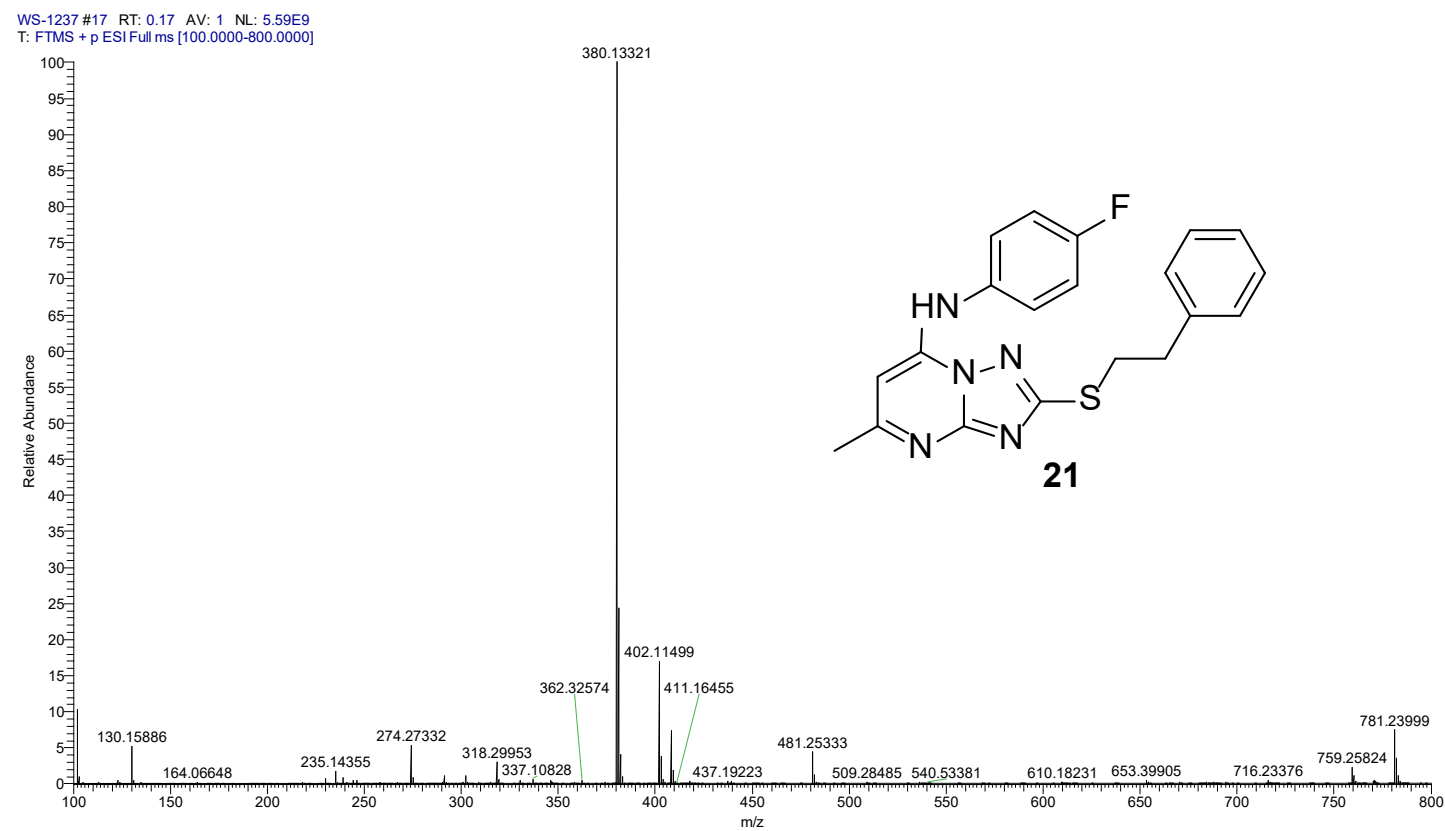

HRMS (ESI): m/z calcd for $\mathrm{C}_{21} \mathrm{H}_{21} \mathrm{FN}_{5} \mathrm{~S}(\mathrm{M}+\mathrm{H})^{+}, 394.1502$; found, 394.1489.

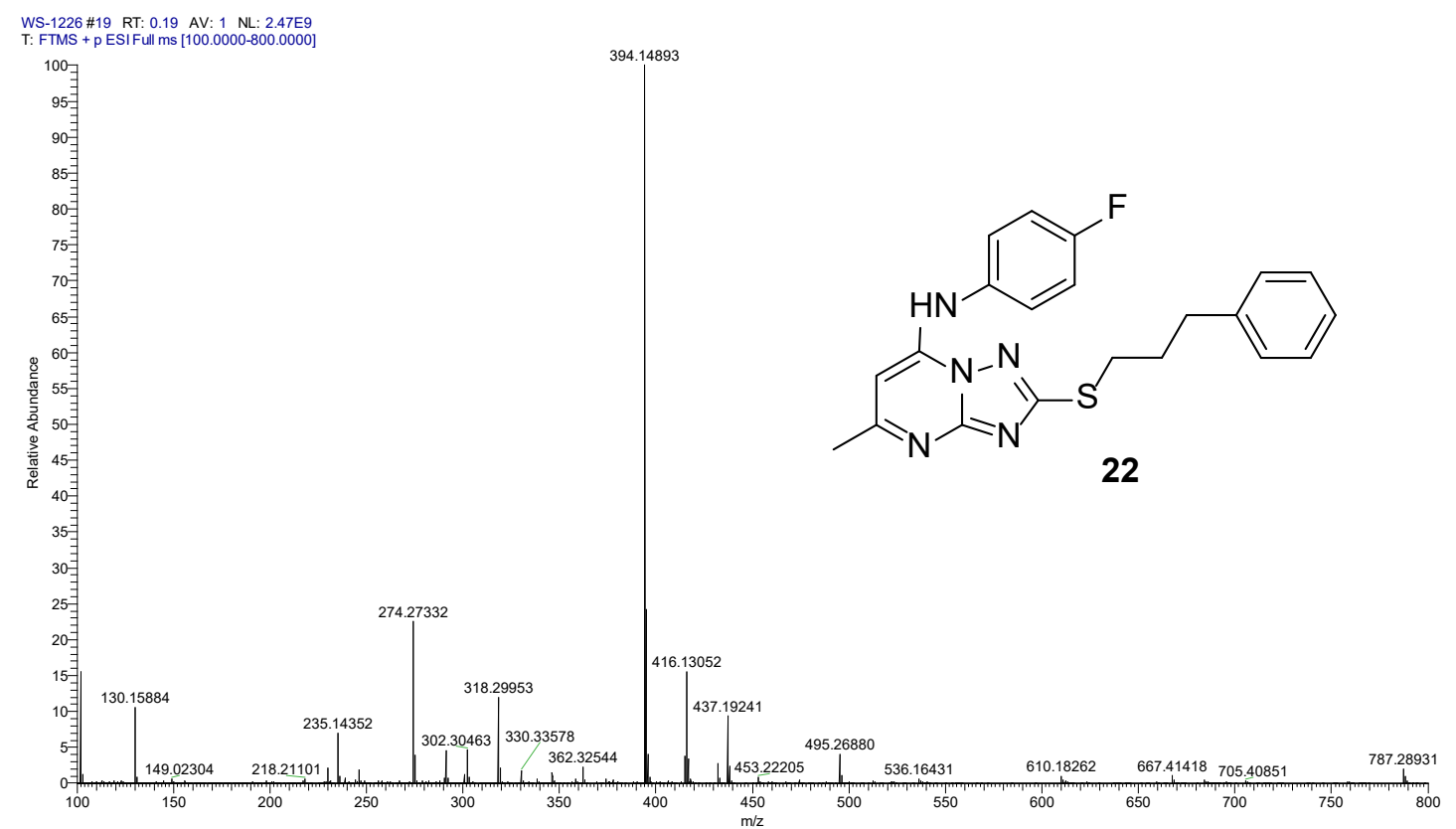


HRMS (ESI): $\mathrm{m} / \mathrm{z}$ calcd for $\mathrm{C}_{15} \mathrm{H}_{17} \mathrm{FN}_{5} \mathrm{~S}(\mathrm{M}+\mathrm{H})^{+}, 318.1189$; found, 318.1178 .

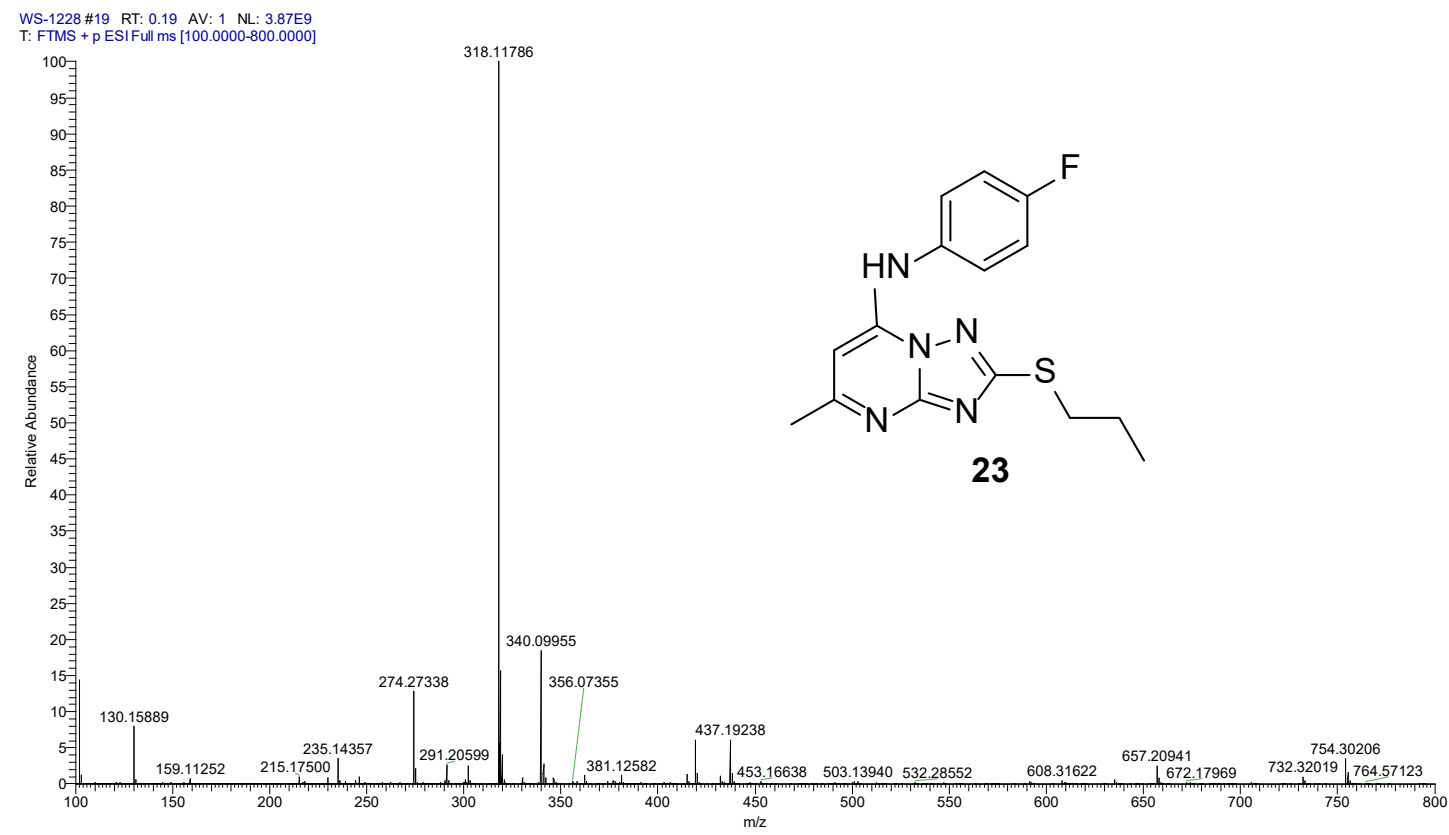

HRMS (ESI): $\mathrm{m} / \mathrm{z}$ calcd for $\mathrm{C}_{15} \mathrm{H}_{15} \mathrm{FN}_{5} \mathrm{~S}(\mathrm{M}+\mathrm{H})^{+}, 316.1032$; found, 316.1031 .

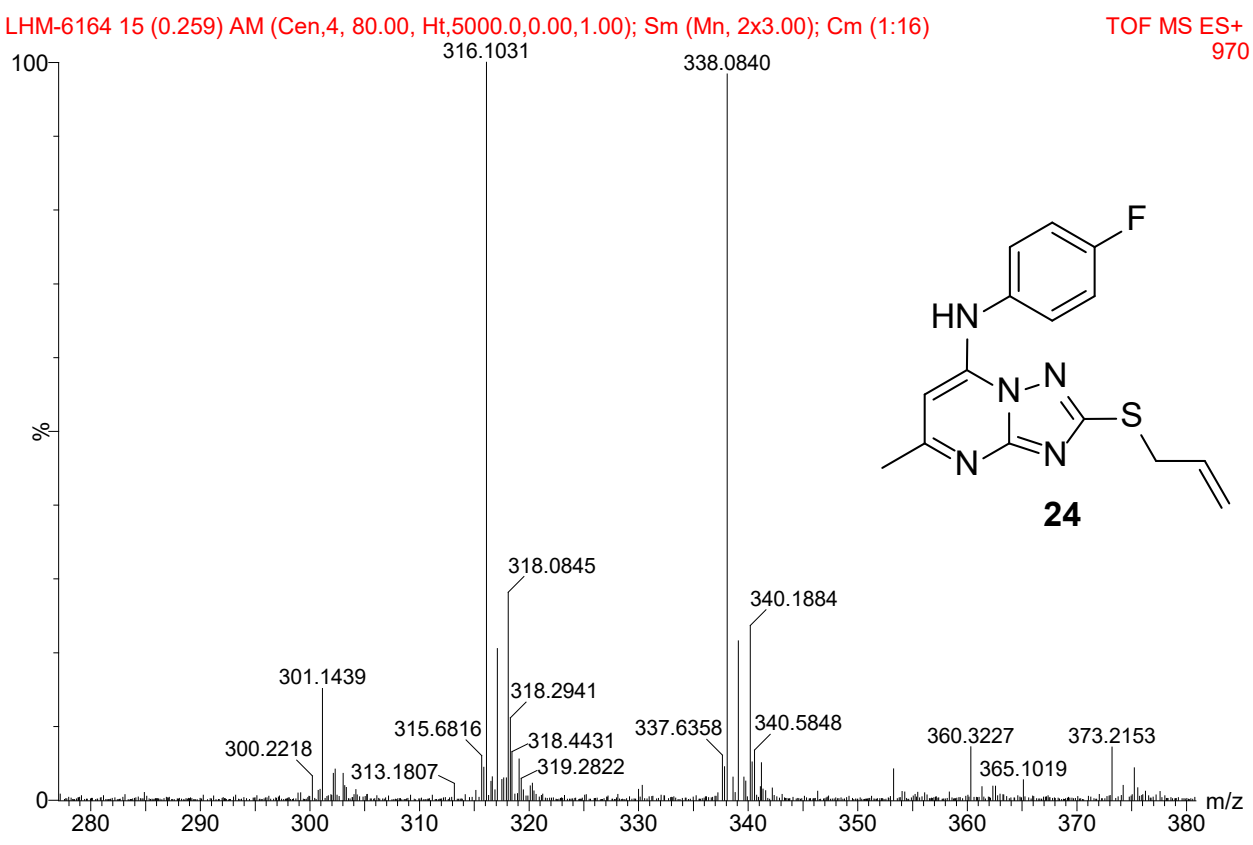


HRMS (ESI): $\mathrm{m} / \mathrm{z}$ calcd for $\mathrm{C}_{15} \mathrm{H}_{13} \mathrm{FN}_{5} \mathrm{~S}(\mathrm{M}+\mathrm{H})^{+}, 314.0876$; found, 314.0877 .

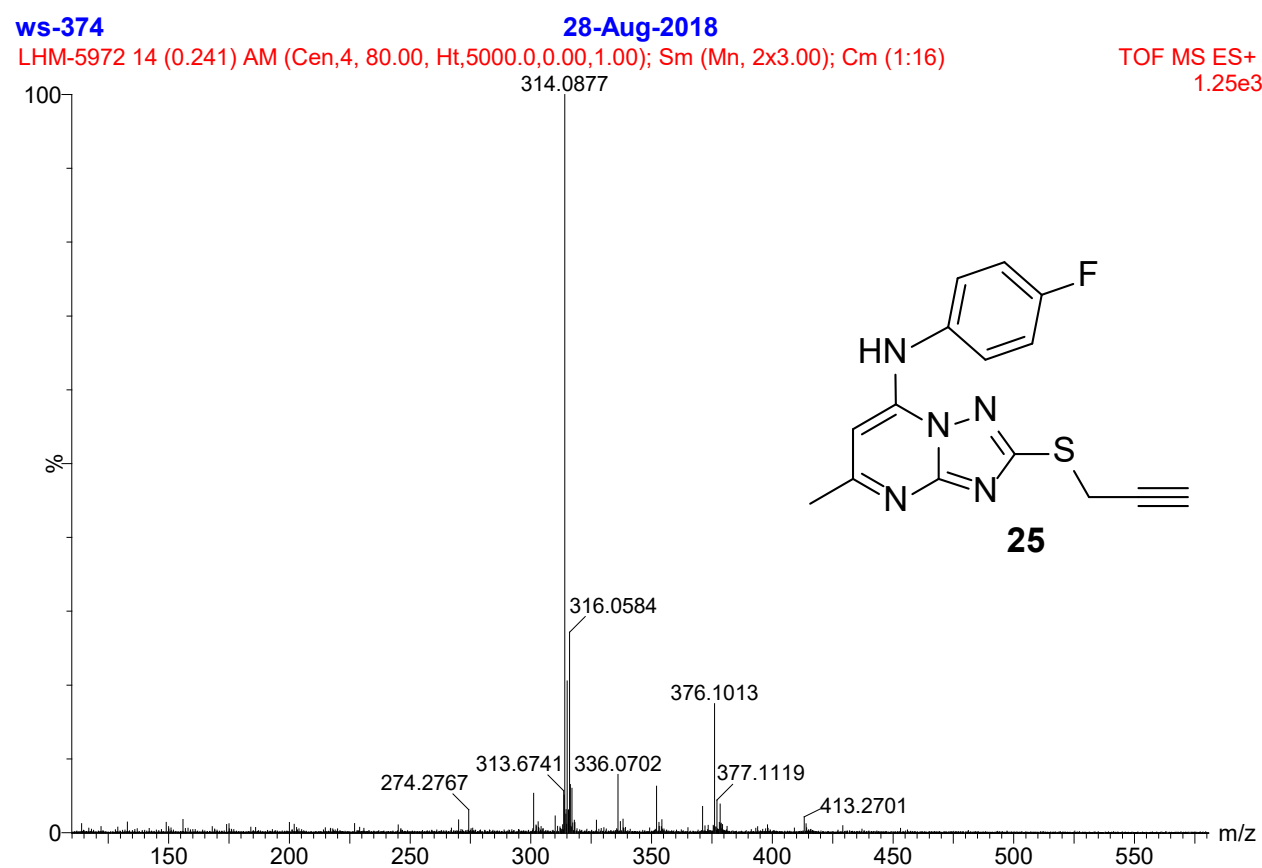

HRMS (ESI): $\mathrm{m} / \mathrm{z}$ calcd for $\mathrm{C}_{16} \mathrm{H}_{17} \mathrm{FN}_{5} \mathrm{~S}(\mathrm{M}+\mathrm{H})^{+}, 330.1189$; found, 330.1177 .

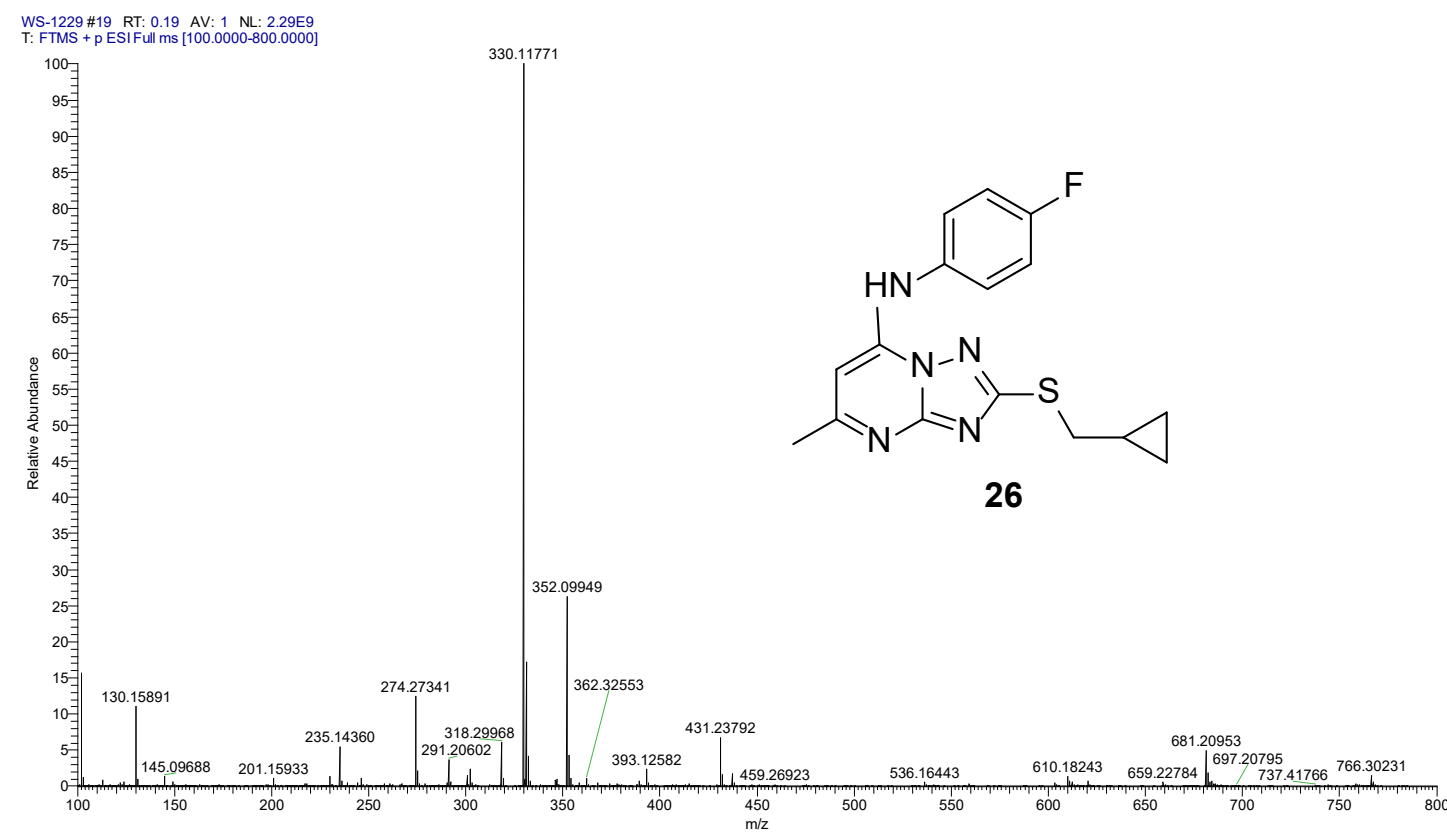


HRMS (ESI): m/z calcd for $\mathrm{C}_{20} \mathrm{H}_{15} \mathrm{FN}_{7} \mathrm{~S}(\mathrm{M}-\mathrm{H})^{+}, 404.1094$; found, 404.1102 .

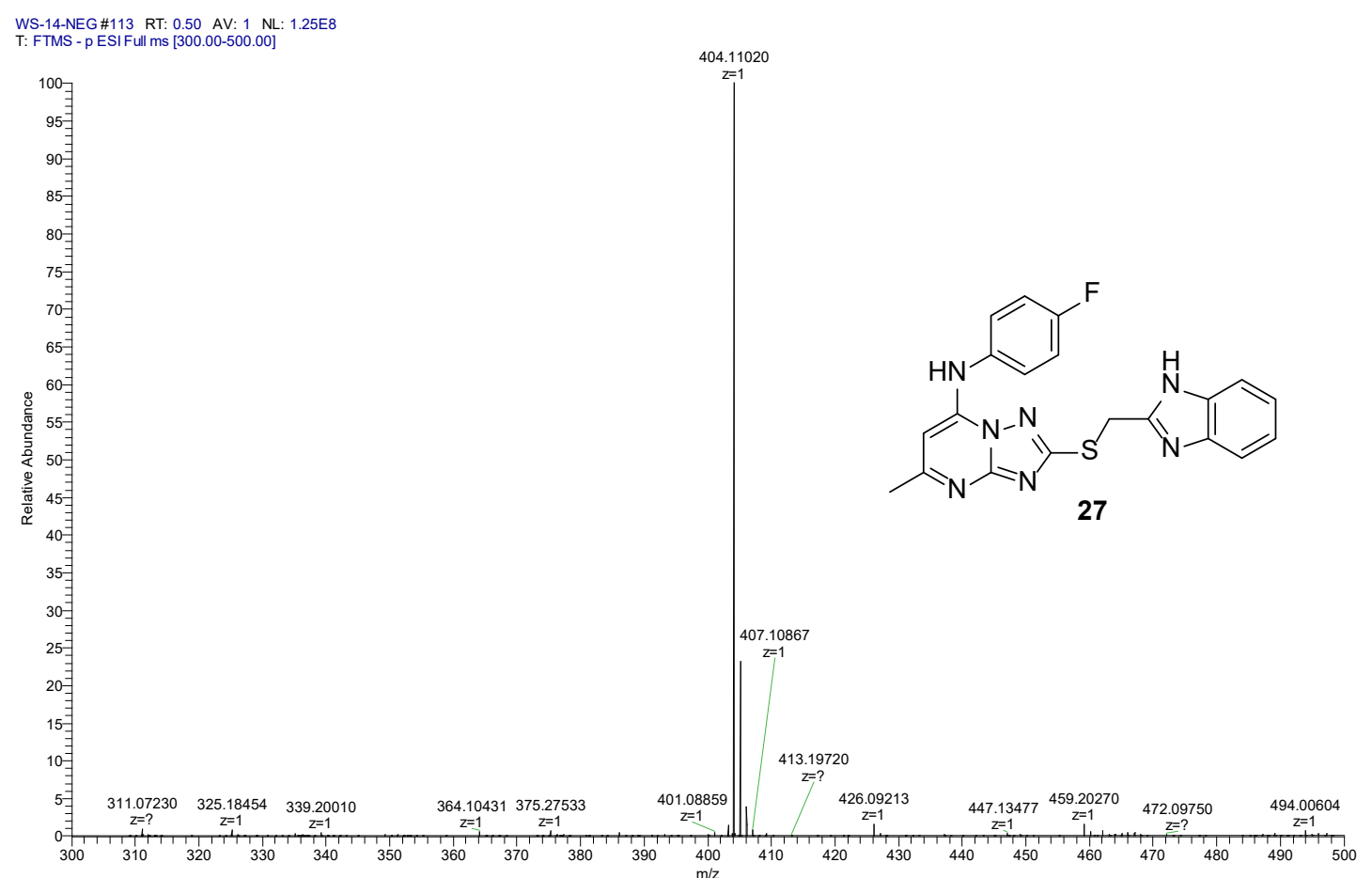

HRMS (ESI): $\mathrm{m} / \mathrm{z}$ calcd for $\mathrm{C}_{21} \mathrm{H}_{16} \mathrm{ClFN}_{5} \mathrm{~S}_{2}(\mathrm{M}+\mathrm{H})^{+}, 456.0520$; found, 456.0503 .

WS-1227 \#19 RT: 0.19 AV: 1 NL: 3.30 EE
T: FTMS + p ESIFull ms [100.0000-800.0000]

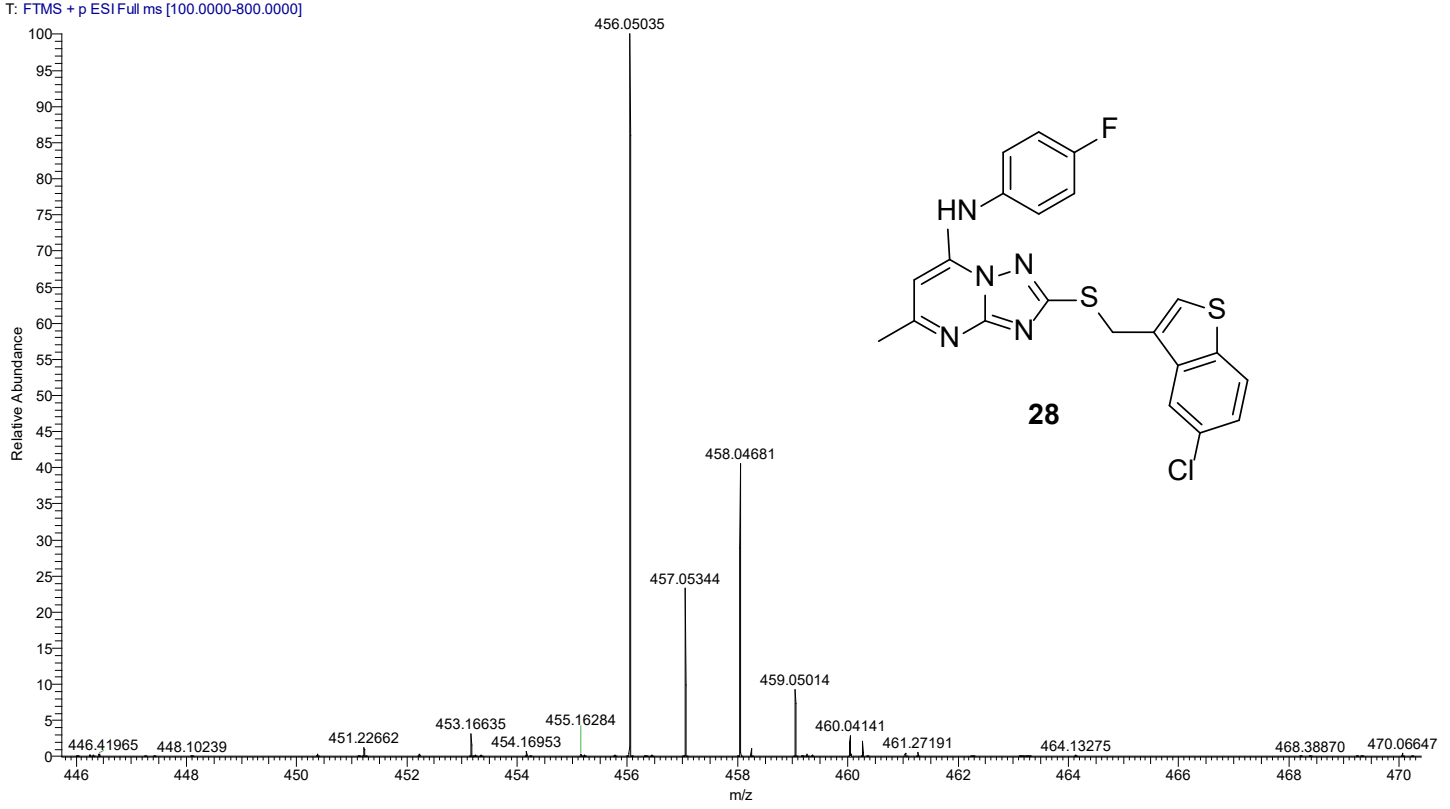


HRMS (ESI): m/z calcd for $\mathrm{C}_{20} \mathrm{H}_{17} \mathrm{FN}_{5} \mathrm{OS}(\mathrm{M}+\mathrm{H})^{+}, 394.1138$; found,394.1137.
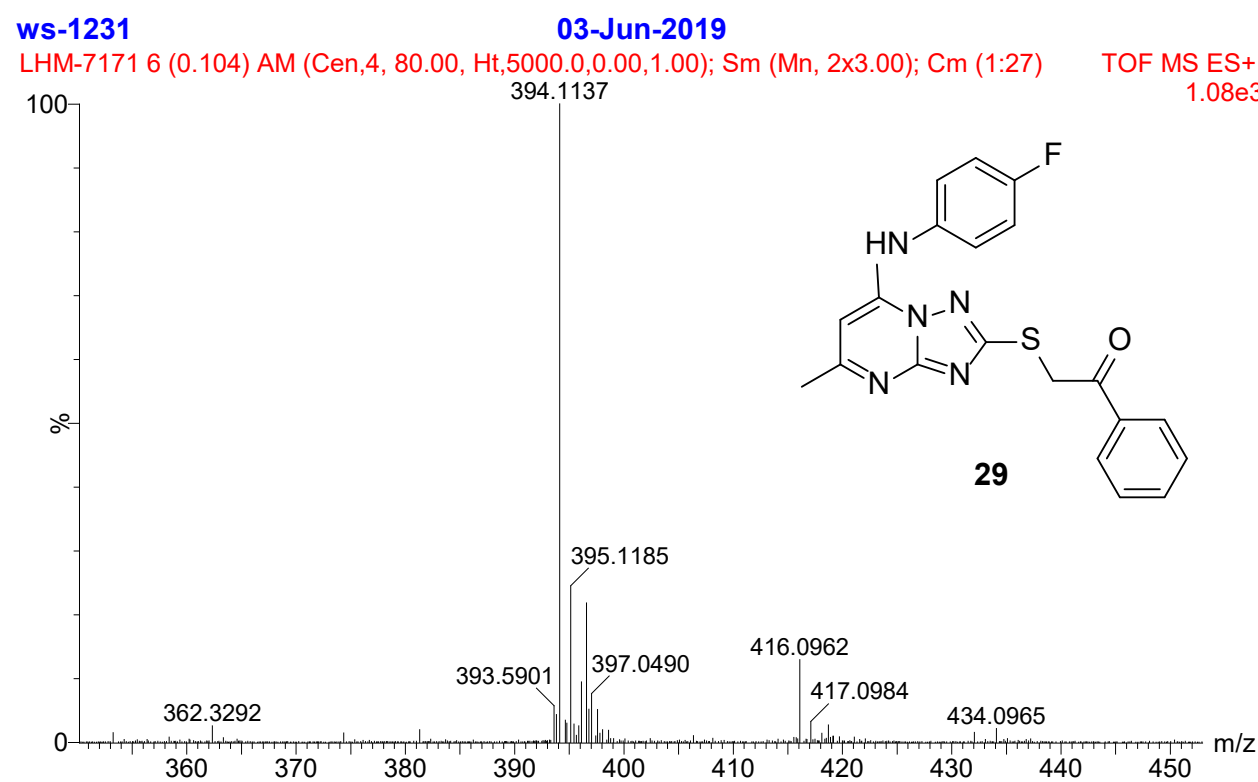

HRMS (ESI): m/z calcd for $\mathrm{C}_{20} \mathrm{H}_{17} \mathrm{FN}_{7} \mathrm{~S}(\mathrm{M}+\mathrm{H})^{+}, 406.1250$; found, 406.1249.

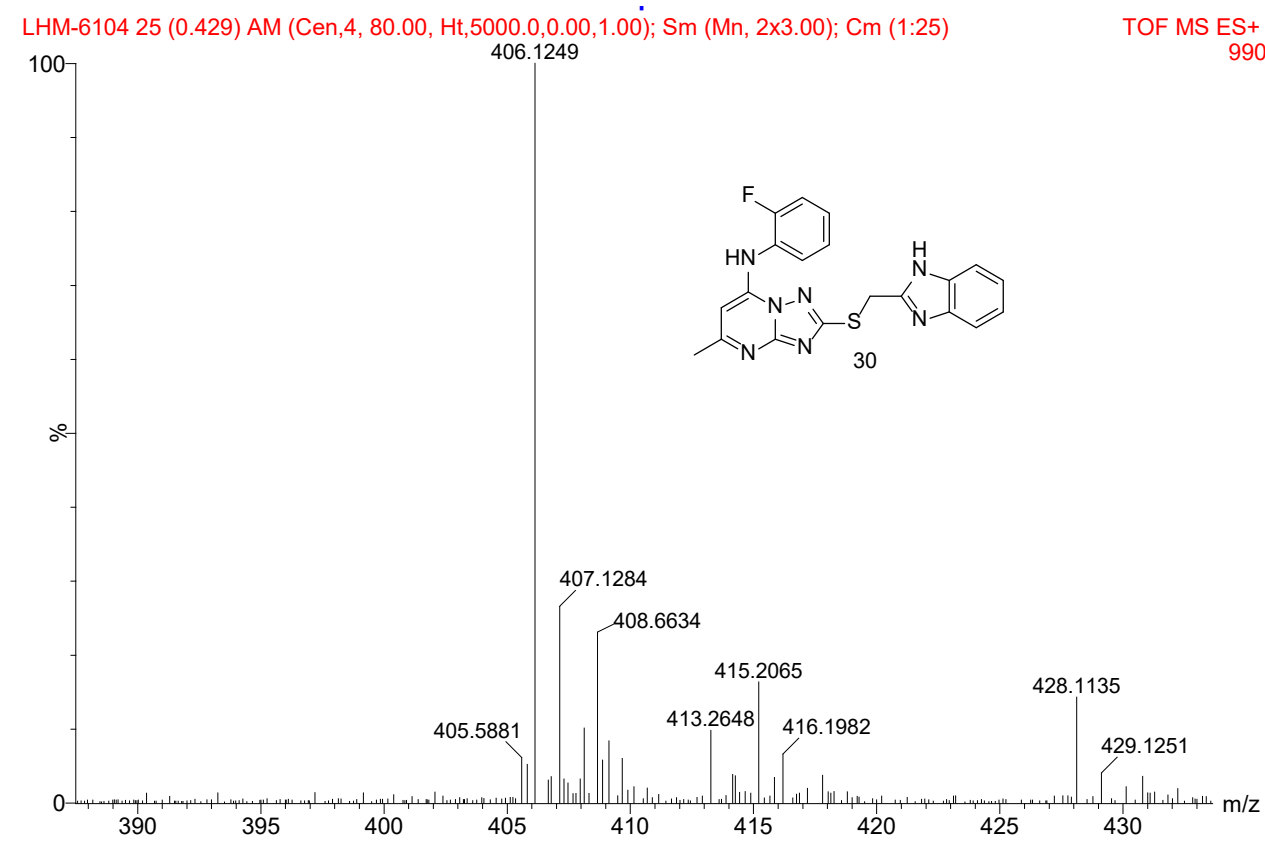


HRMS (ESI): $\mathrm{m} / \mathrm{z}$ calcd for $\mathrm{C}_{20} \mathrm{H}_{17} \mathrm{FN}_{7} \mathrm{~S}(\mathrm{M}+\mathrm{H})^{+}, 406.1250$; found, 406.1250 .

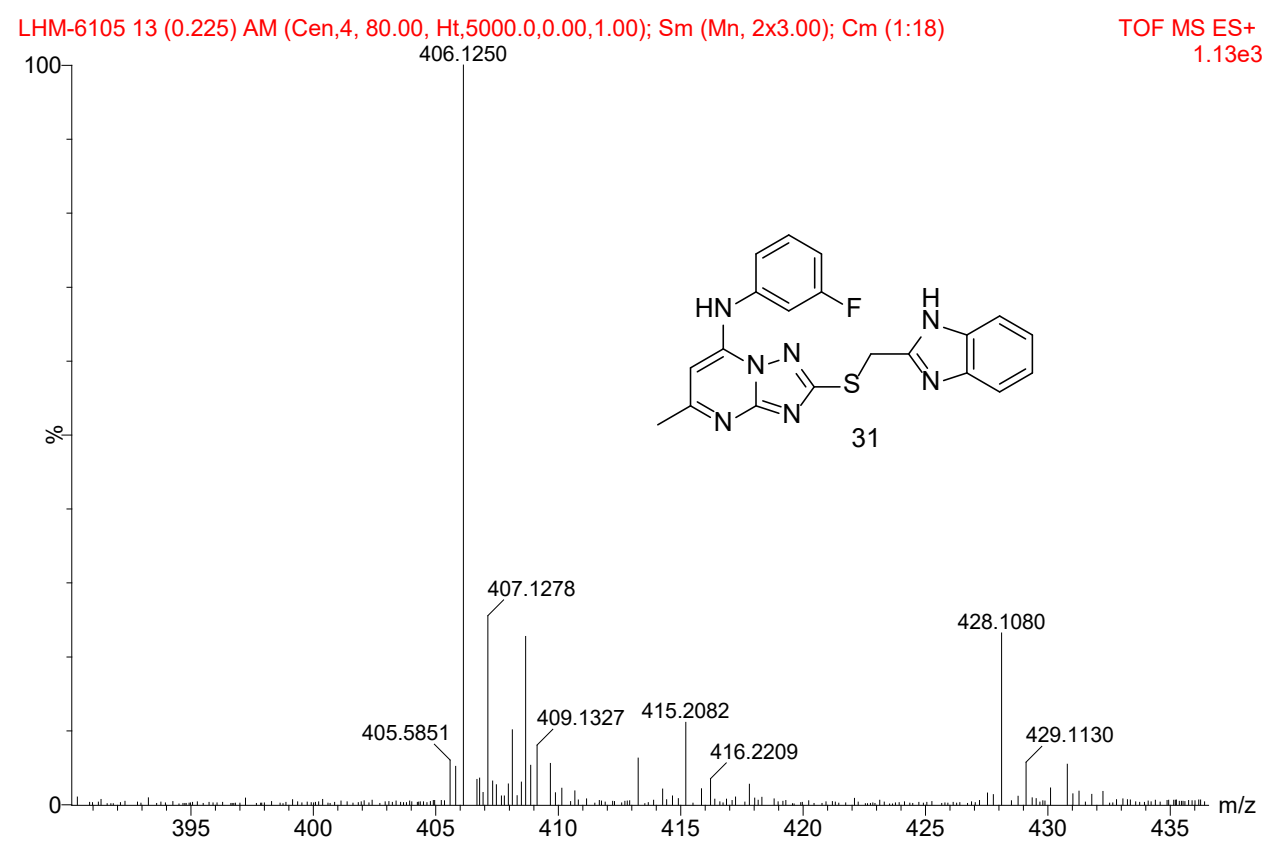

HRMS (ESI): $\mathrm{m} / \mathrm{z}$ calcd for $\mathrm{C}_{20} \mathrm{H}_{16} \mathrm{~F}_{2} \mathrm{~N}_{7} \mathrm{~S}(\mathrm{M}+\mathrm{H})^{+}, 424.1156$; found, 424.1157.

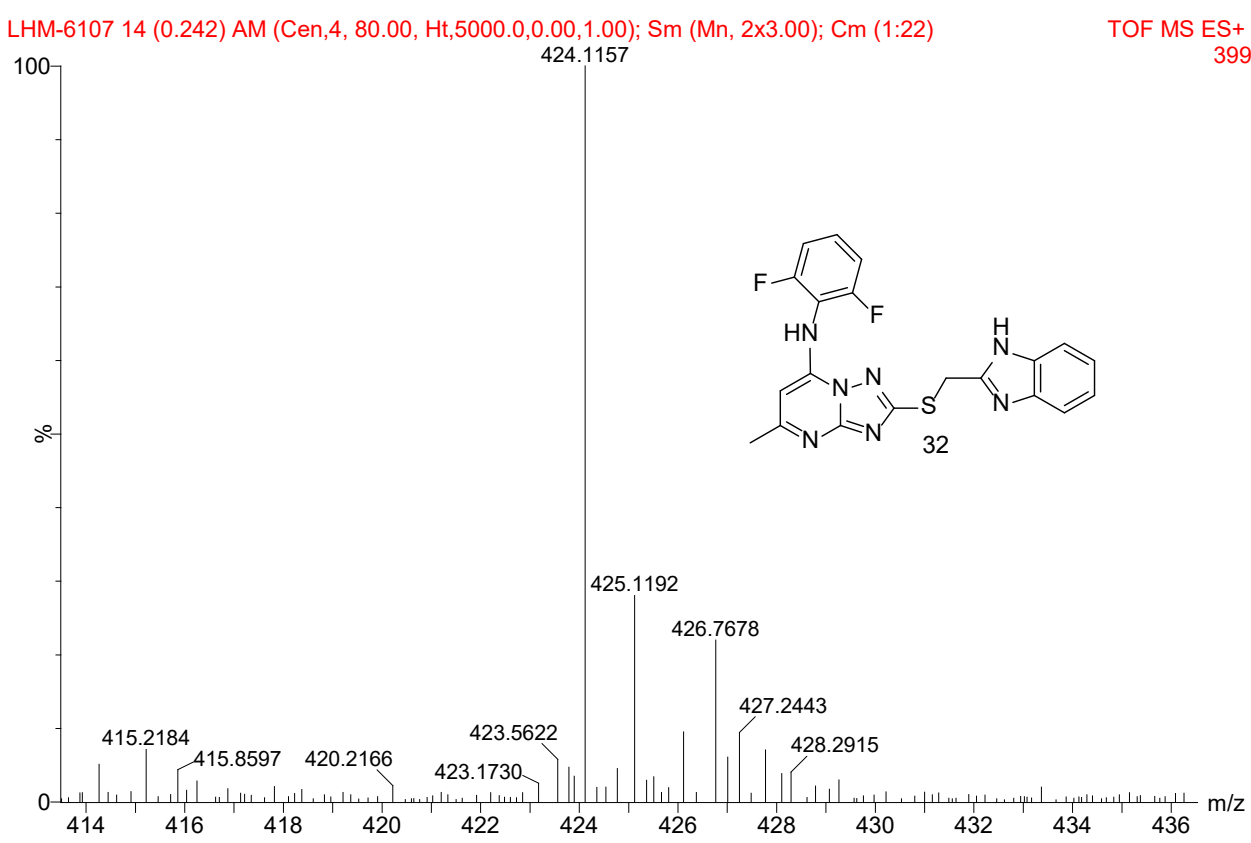


HRMS (ESI): $\mathrm{m} / \mathrm{z}$ calcd for $\mathrm{C}_{20} \mathrm{H}_{16} \mathrm{ClFN}_{7} \mathrm{~S}(\mathrm{M}+\mathrm{H})^{+}$, 440.0860; found, 440.0860.

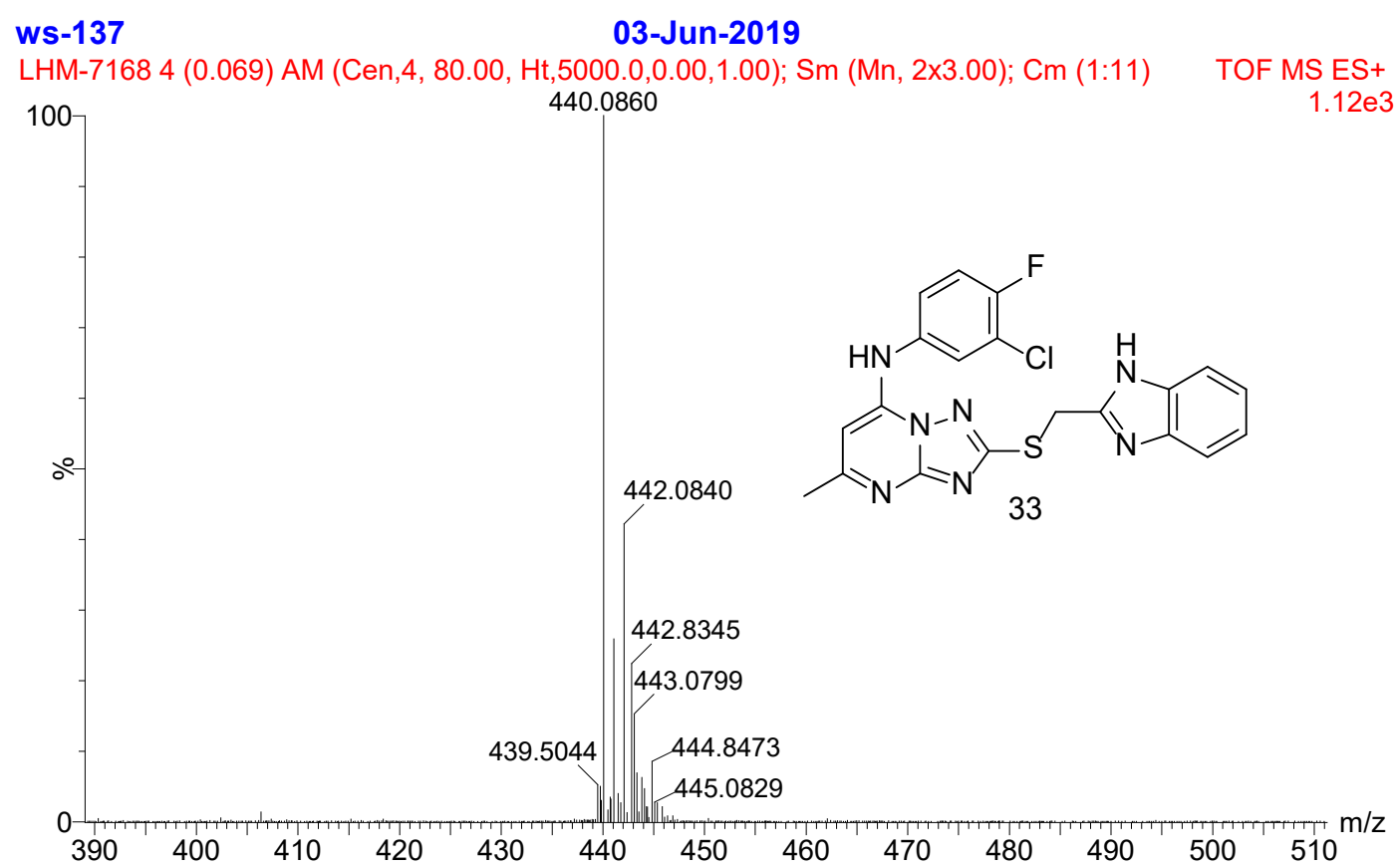

HRMS (ESI): $\mathrm{m} / \mathrm{z}$ calcd for $\mathrm{C}_{20} \mathrm{H}_{15} \mathrm{~F}_{3} \mathrm{~N}_{7} \mathrm{~S}(\mathrm{M}+\mathrm{H})^{+}, 442.1062$; found, 442.1062 .

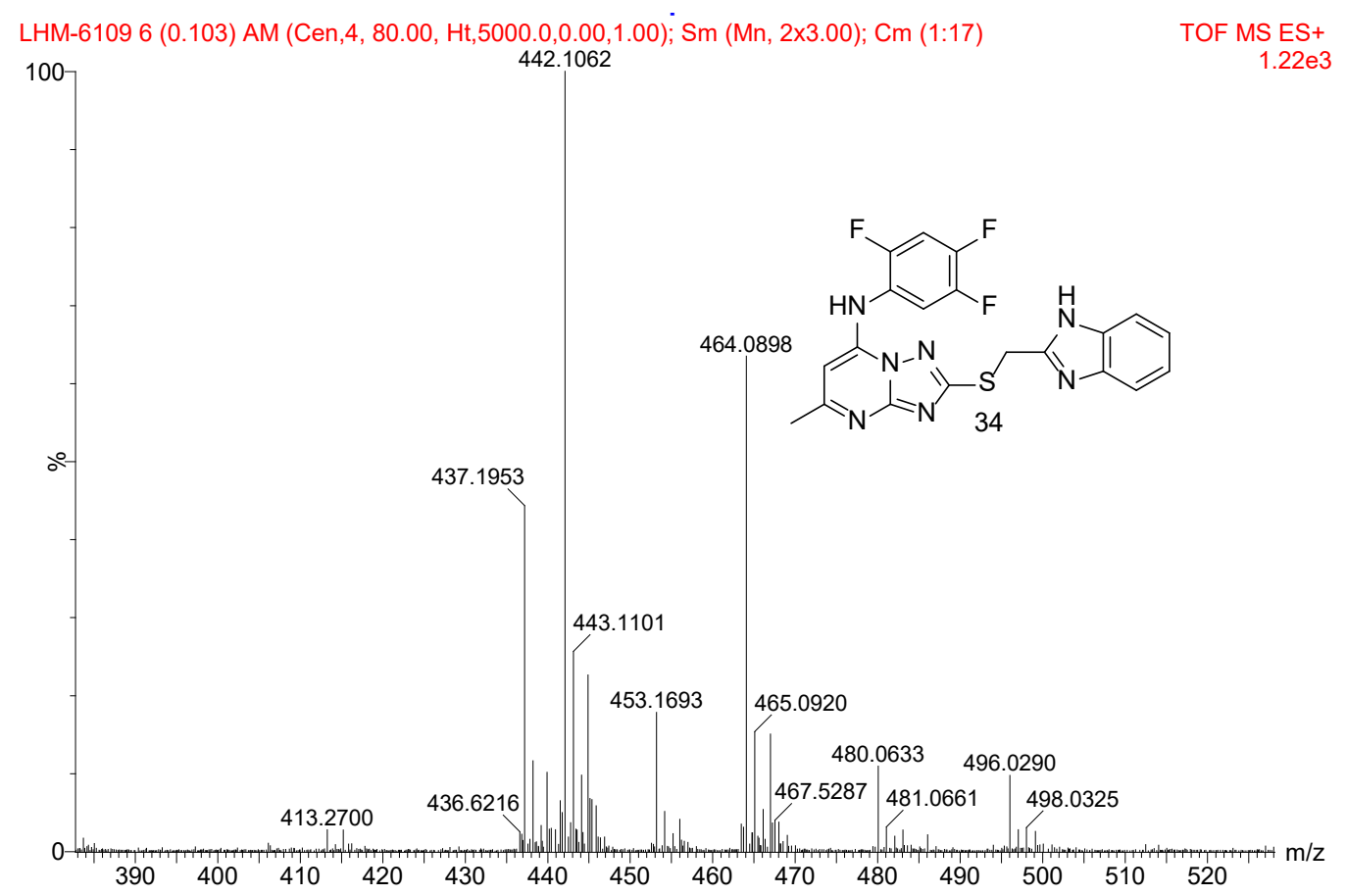


HRMS (ESI): $\mathrm{m} / \mathrm{z}$ calcd for $\mathrm{C}_{21} \mathrm{H}_{19} \mathrm{FN}_{7} \mathrm{~S}(\mathrm{M}+\mathrm{H})^{+}, 420.1407$; found, 420.1407 .

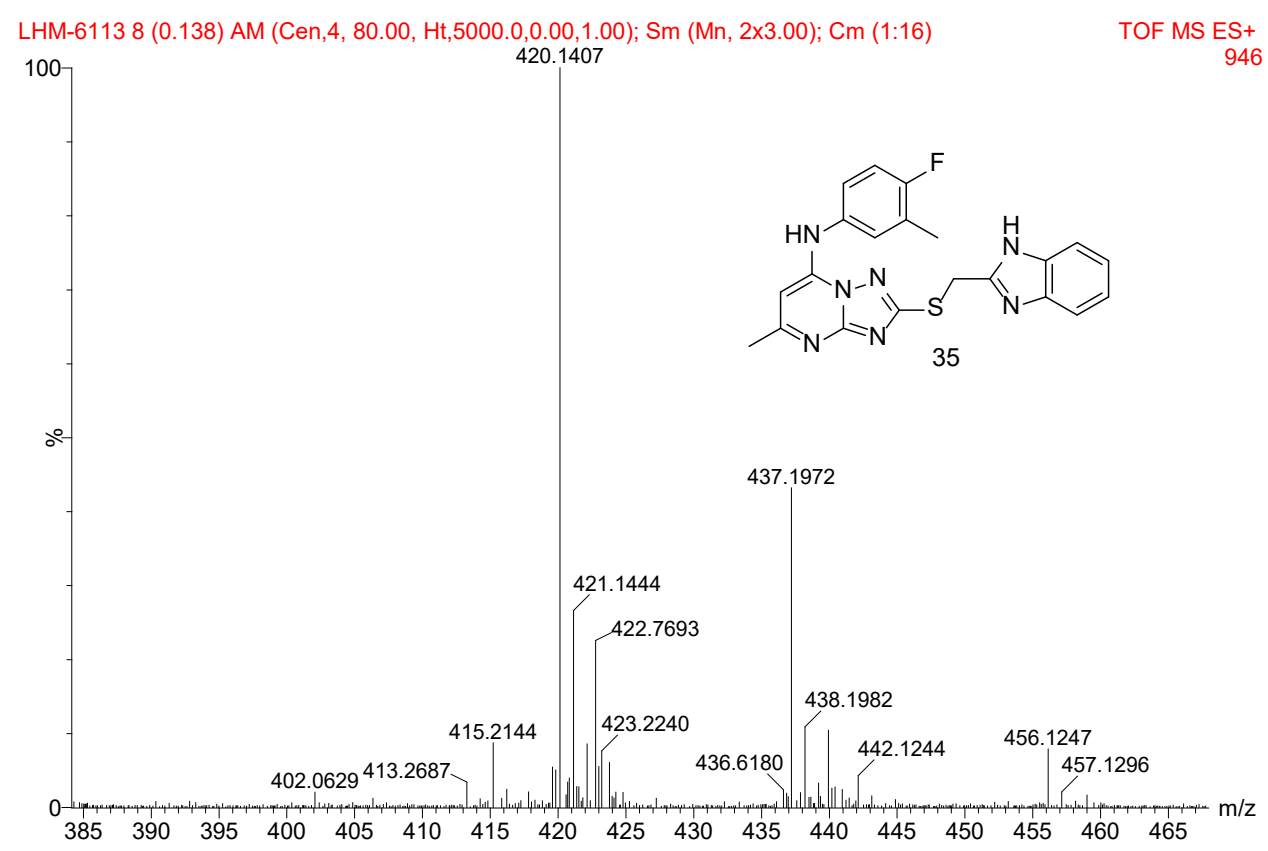

HRMS (ESI): $\mathrm{m} / \mathrm{z}$ calcd for $\mathrm{C}_{21} \mathrm{H}_{19} \mathrm{FN}_{7} \mathrm{~S}(\mathrm{M}+\mathrm{H})^{+}, 420.1407$; found, 420.1408.

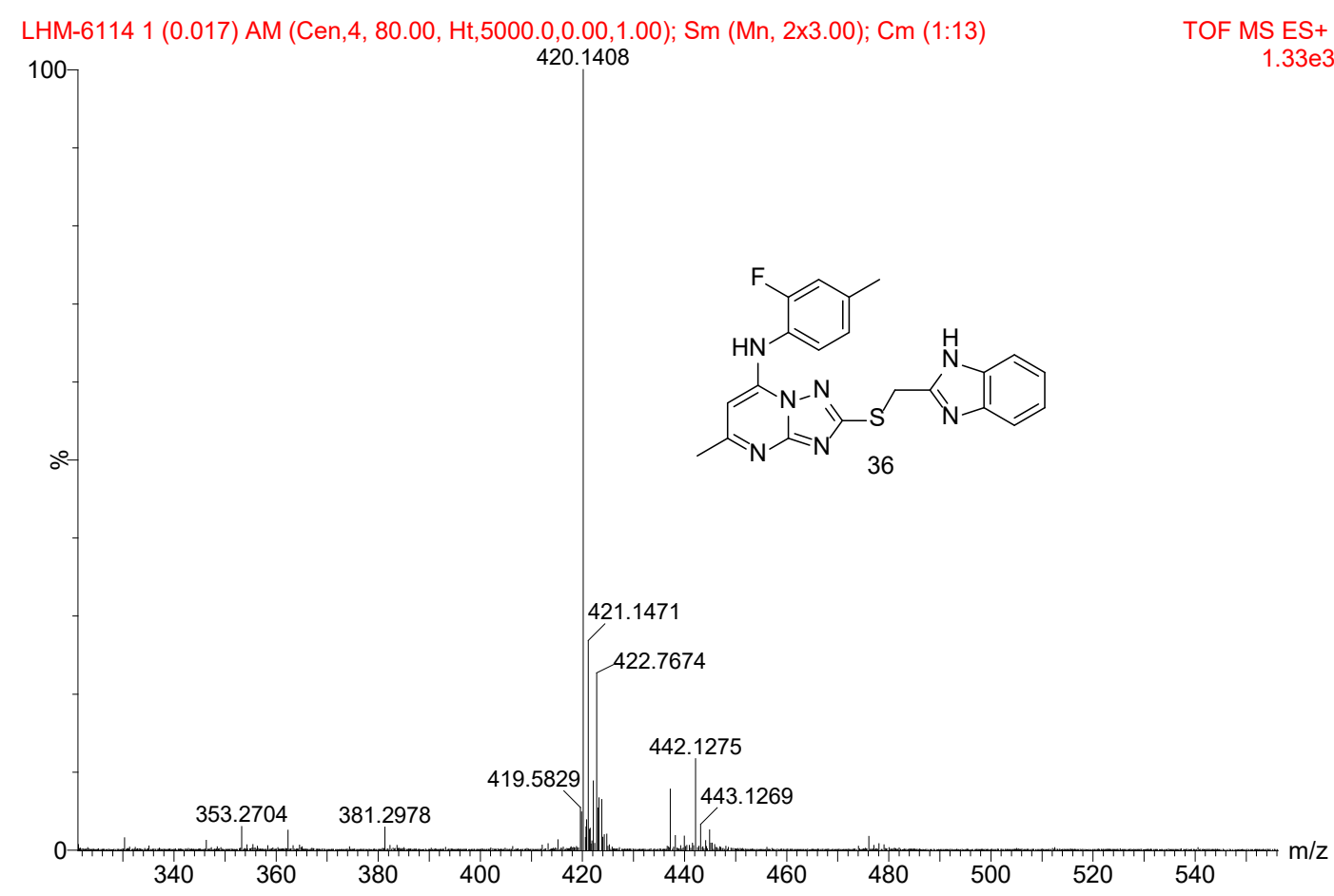


HRMS (ESI): $\mathrm{m} / \mathrm{z}$ calcd for $\mathrm{C}_{21} \mathrm{H}_{17} \mathrm{~F}_{3} \mathrm{~N}_{7} \mathrm{~S}(\mathrm{M}+\mathrm{H})^{+}$, 456.1218; found, 456.1219 .

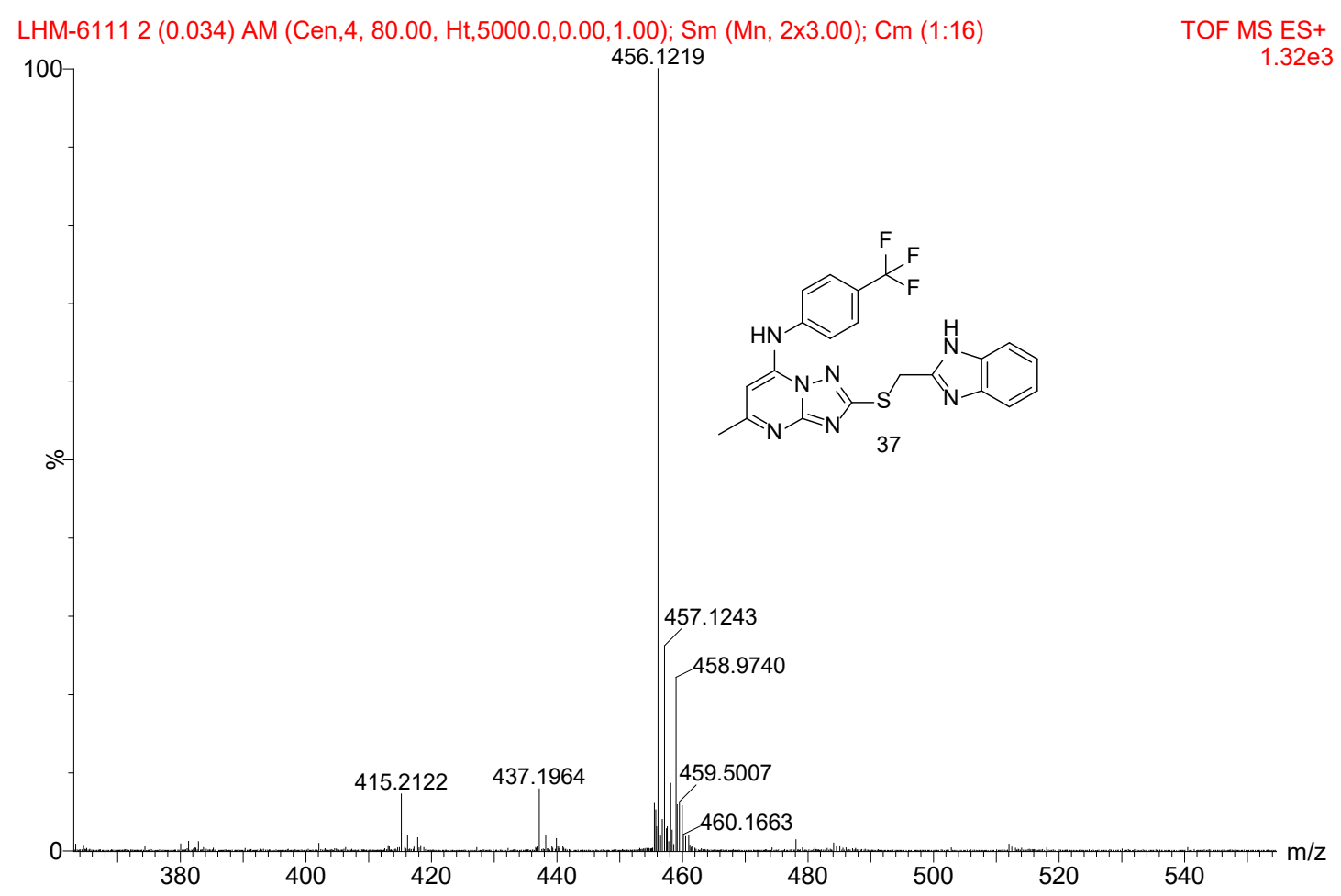

HRMS (ESI): m/z calcd for $\mathrm{C}_{21} \mathrm{H}_{17} \mathrm{~F}_{3} \mathrm{~N}_{7} \mathrm{~S}(\mathrm{M}+\mathrm{H})^{+}$, 456.1218; found, 456.1213 .

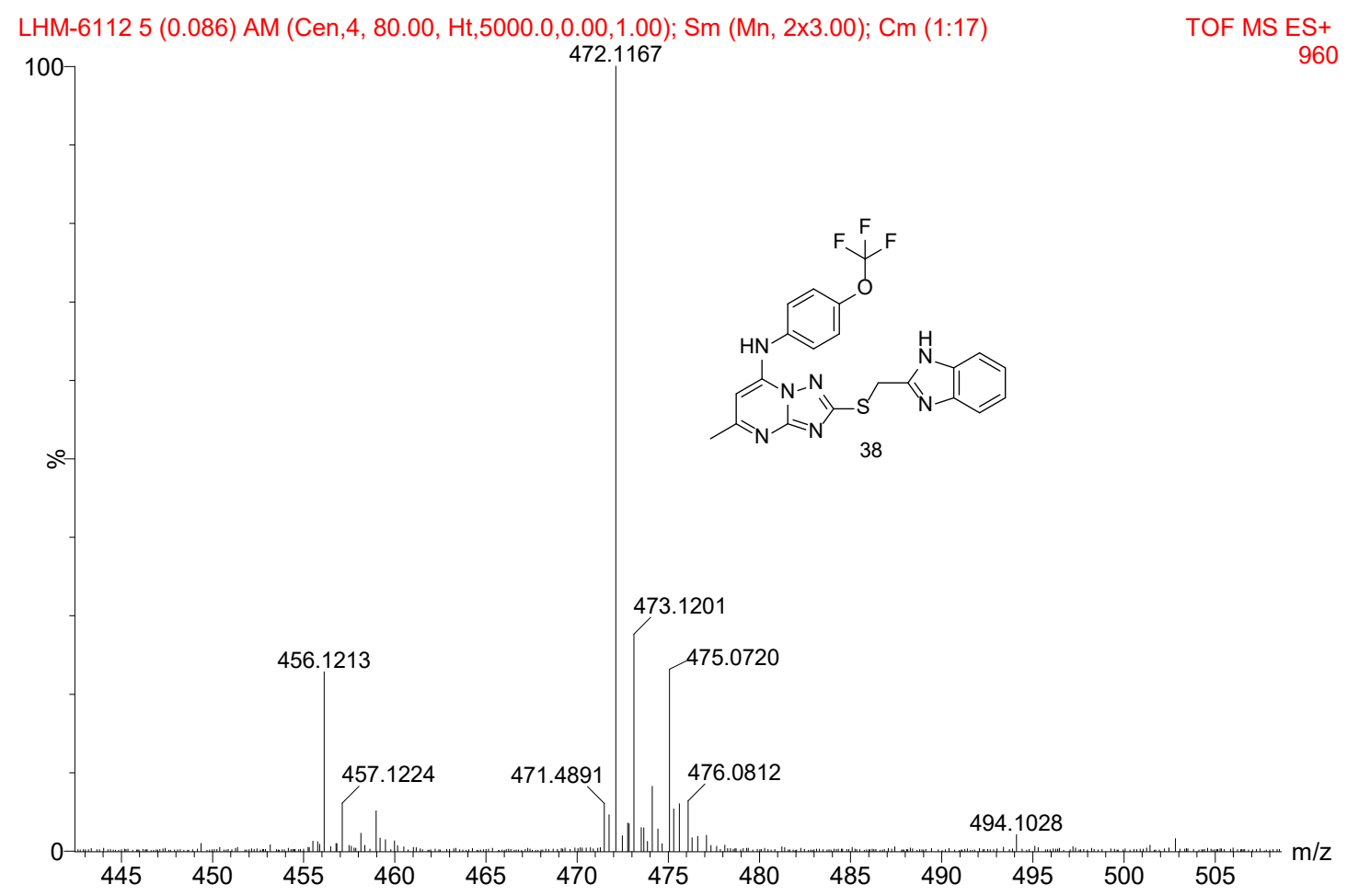


HRMS (ESI): m/z calcd for $\mathrm{C}_{20} \mathrm{H}_{16} \mathrm{~N}_{7} \mathrm{~S}(\mathrm{M}-\mathrm{H})^{+}, 386.1188$; found, 386.1196 .

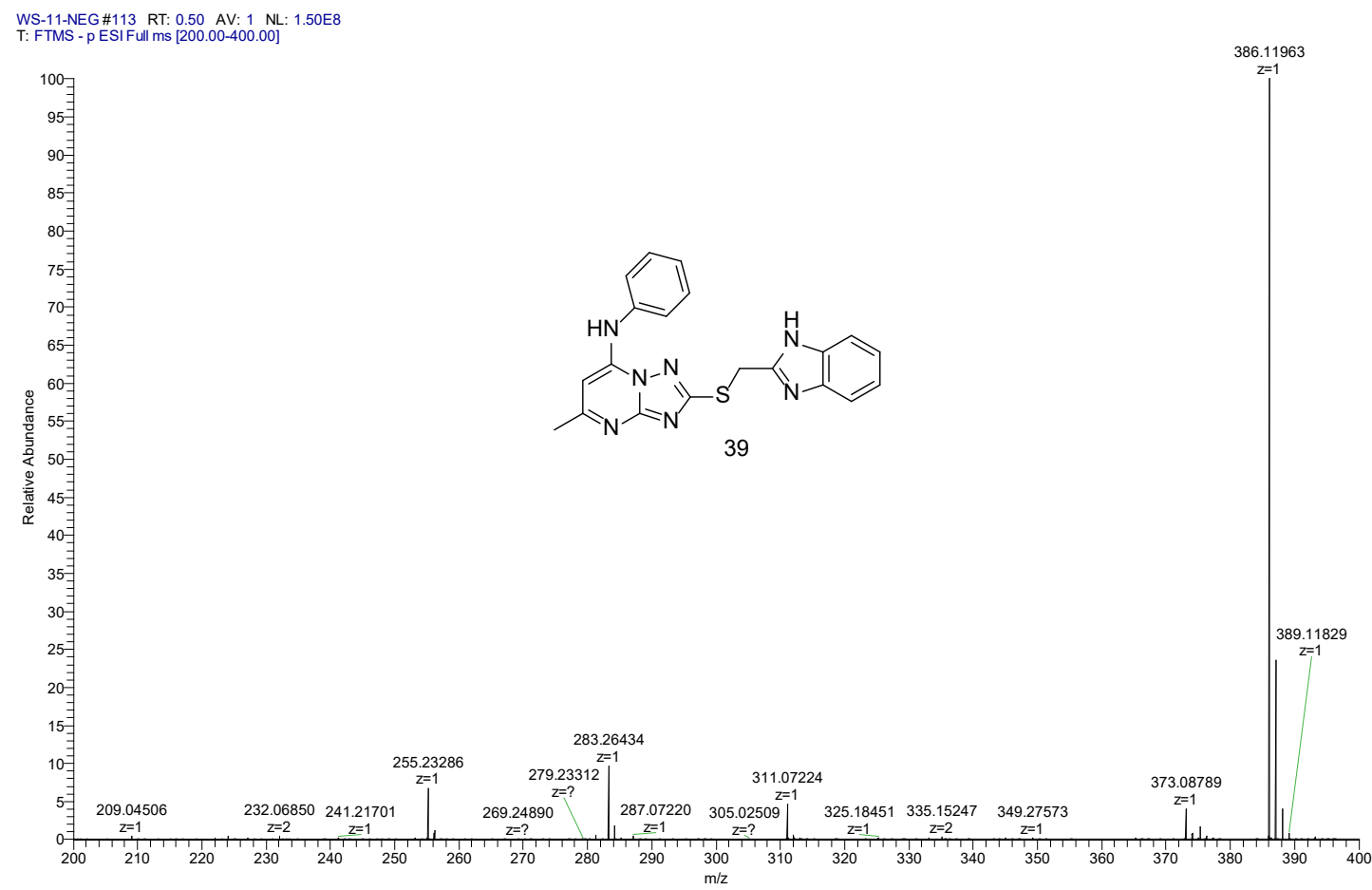

HRMS (ESI): m/z calcd for $\mathrm{C}_{20} \mathrm{H}_{15} \mathrm{ClN}_{7} \mathrm{~S}(\mathrm{M}-\mathrm{H})^{+}$, 420.0798; found, 420.0807 .

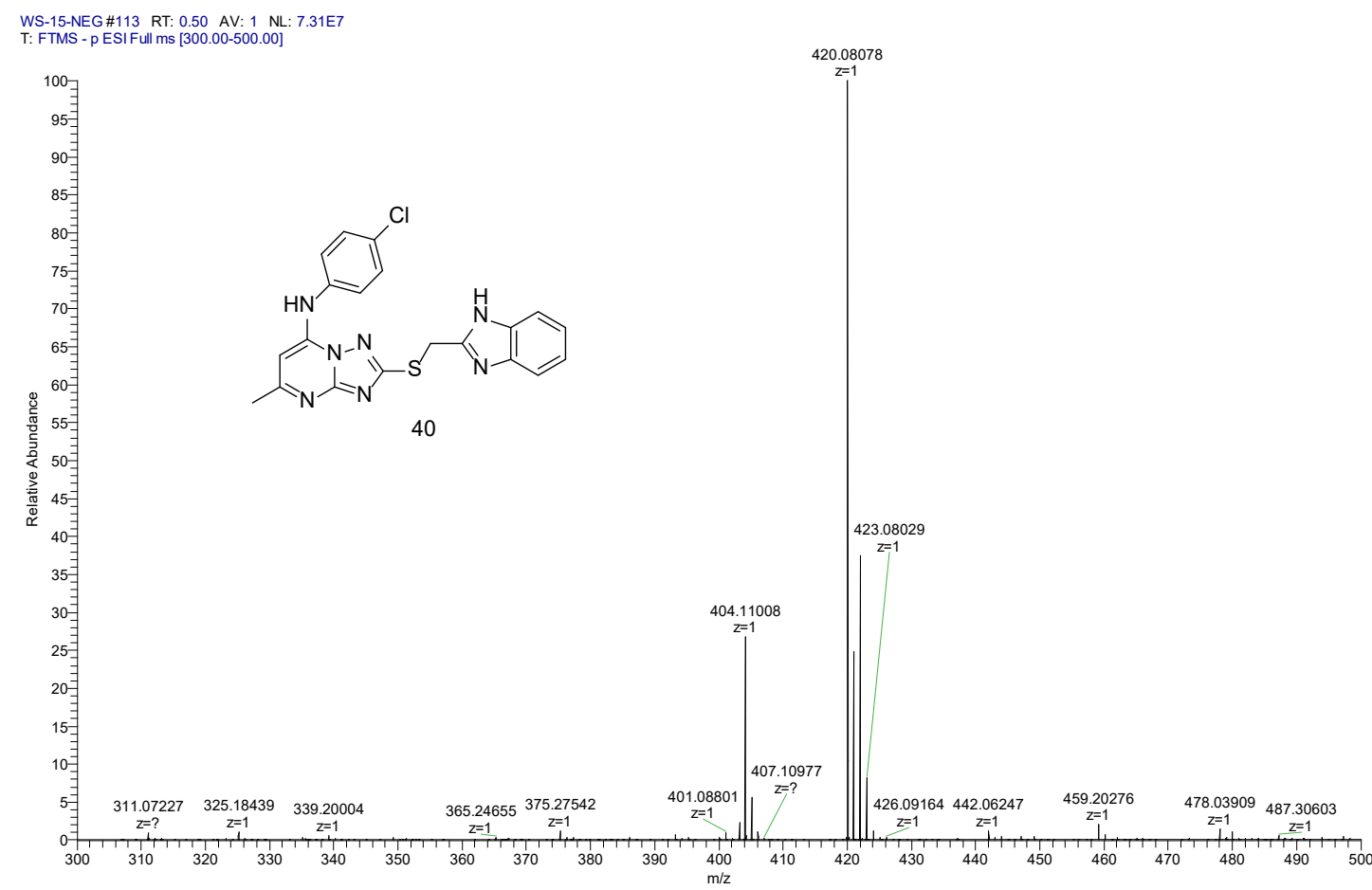


HRMS (ESI): m/z calcd for $\mathrm{C}_{20} \mathrm{H}_{17} \mathrm{BrN}_{7} \mathrm{~S}(\mathrm{M}+\mathrm{H})^{+}$, 466.0450; found, 466.0277.

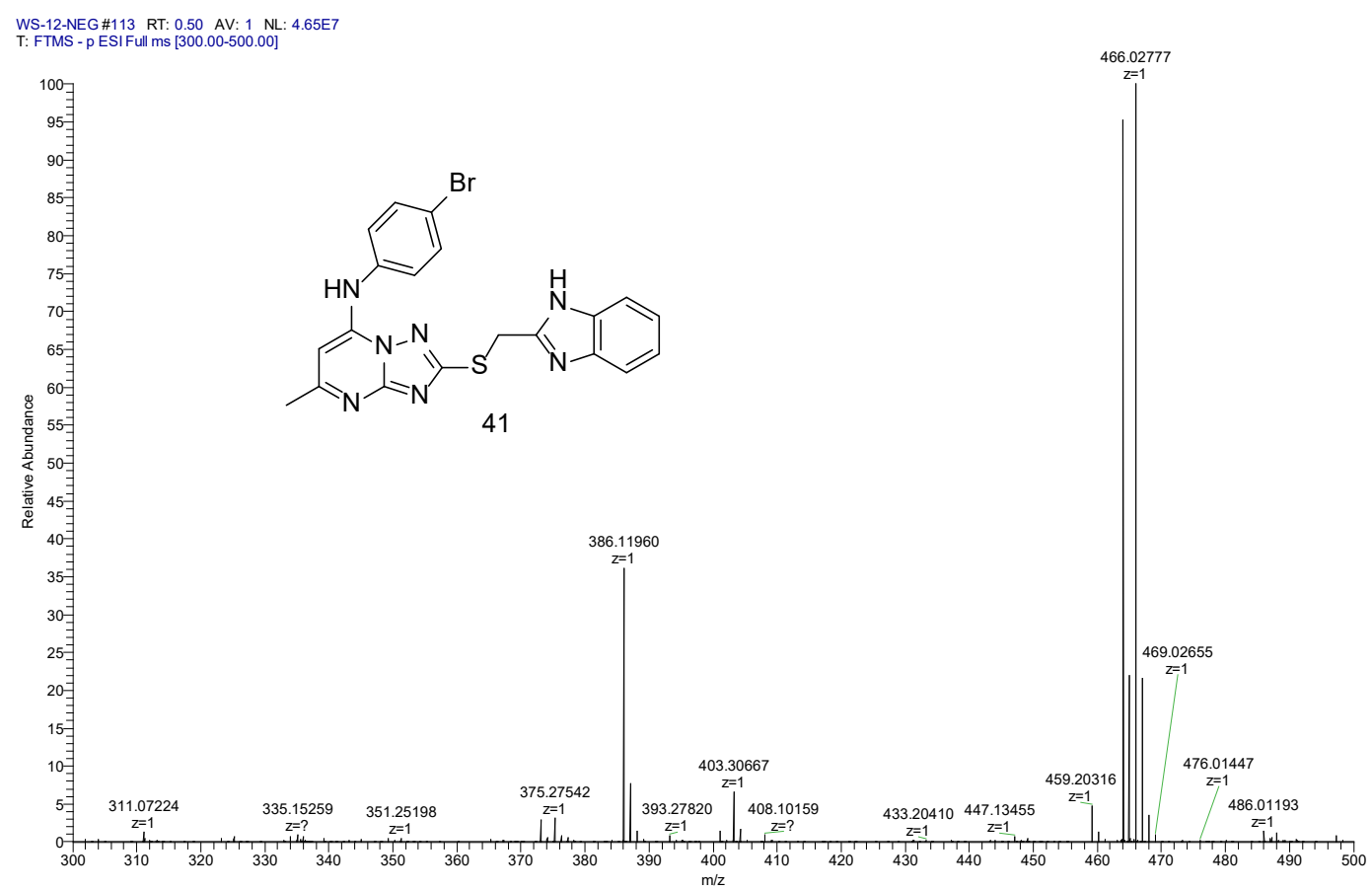

HRMS (ESI): $\mathrm{m} / \mathrm{z}$ calcd for $\mathrm{C}_{21} \mathrm{H}_{16} \mathrm{~N}_{7} \mathrm{O}_{2} \mathrm{~S}(\mathrm{M}-\mathrm{H})^{+}, 430.1086$; found, 430.1094.

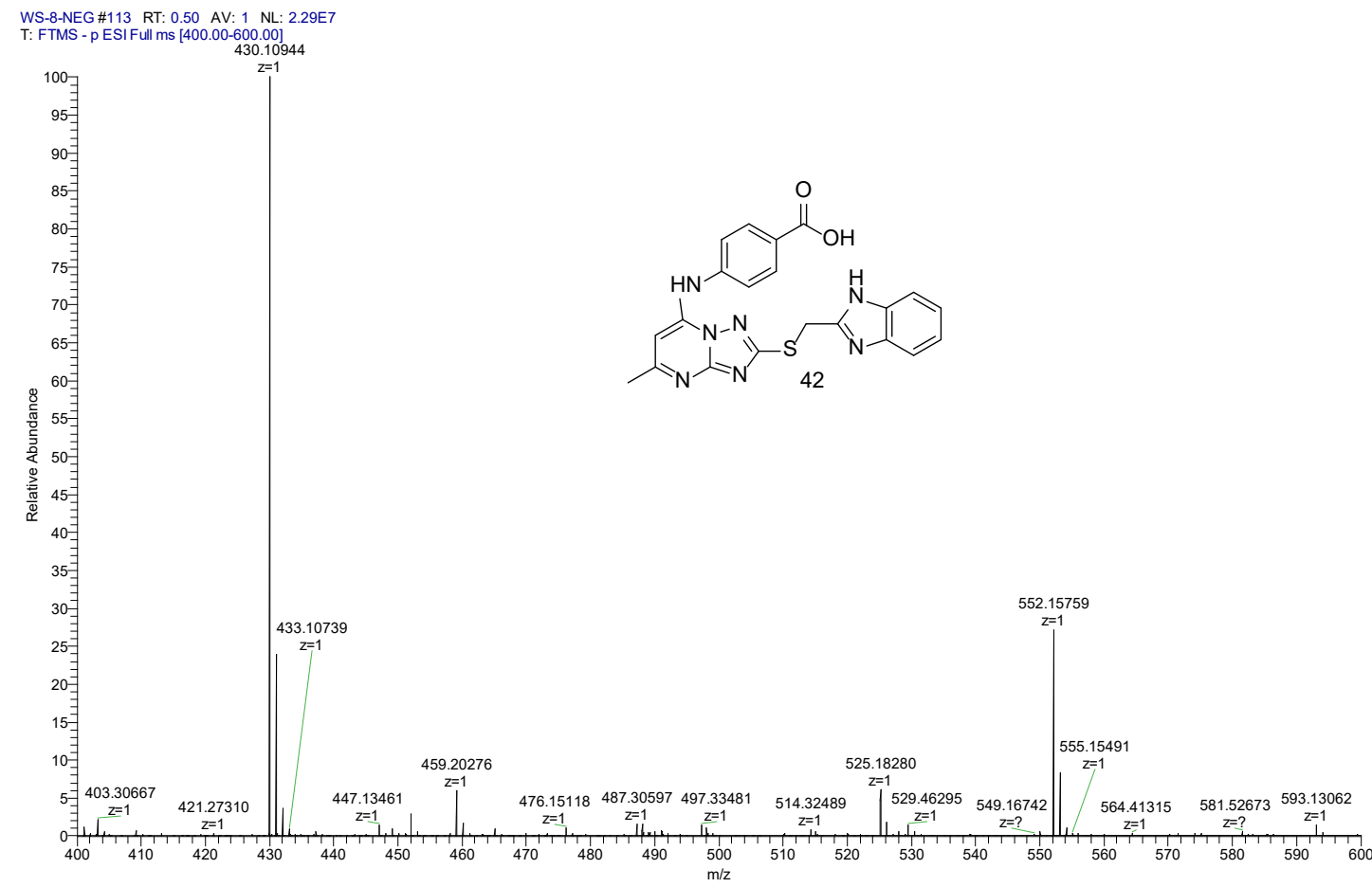


HRMS (ESI): m/z calcd for $\mathrm{C}_{21} \mathrm{H}_{15} \mathrm{~N}_{8} \mathrm{~S}(\mathrm{M}-\mathrm{H})^{+}, 411.1140$; found, 411.1148 .

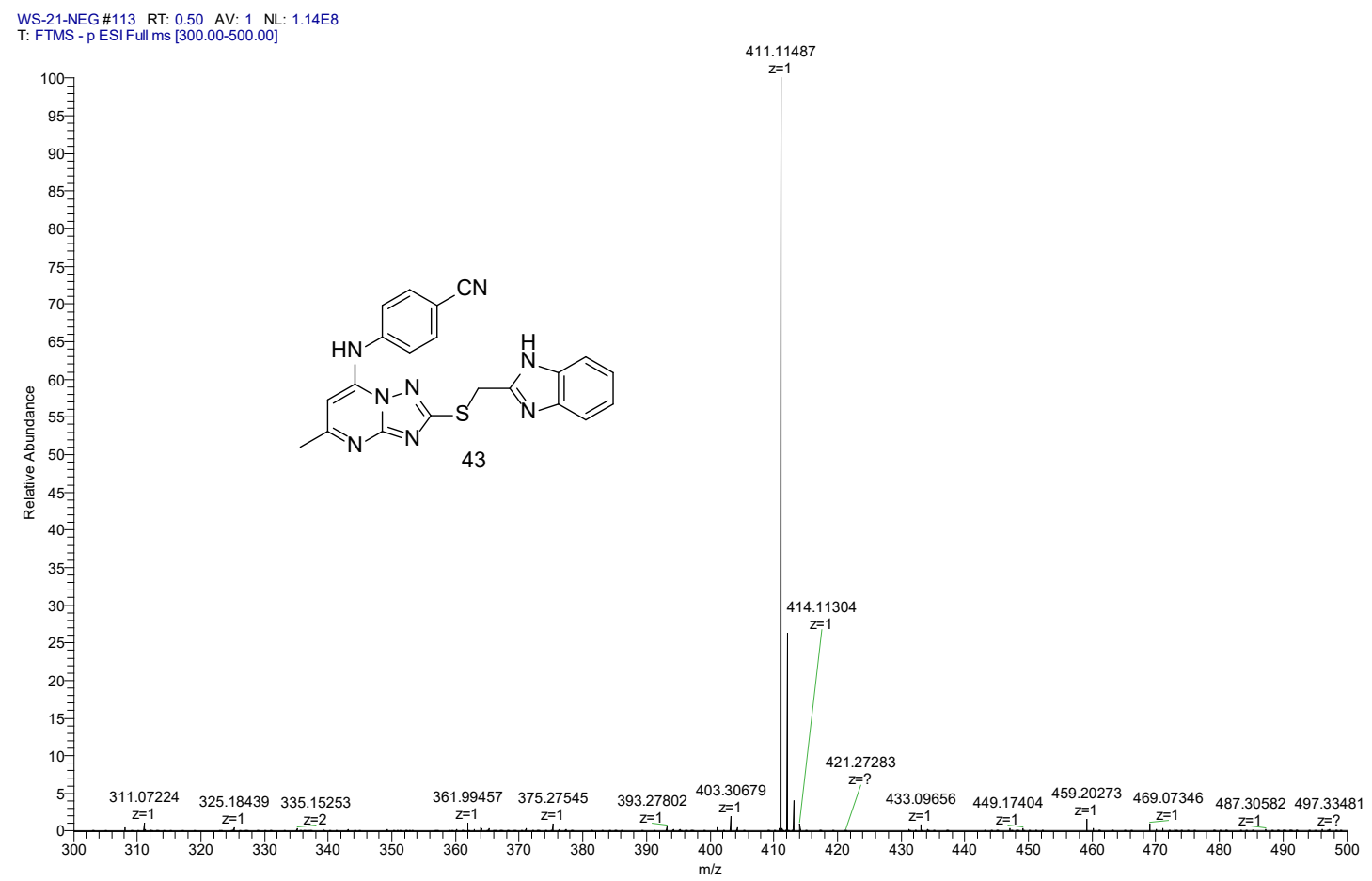

HRMS (ESI): $\mathrm{m} / \mathrm{z}$ calcd for $\mathrm{C}_{22} \mathrm{H}_{20} \mathrm{~N}_{7} \mathrm{OS}(\mathrm{M}+\mathrm{H})^{+}$, 430.1450; found, 430.1451 .

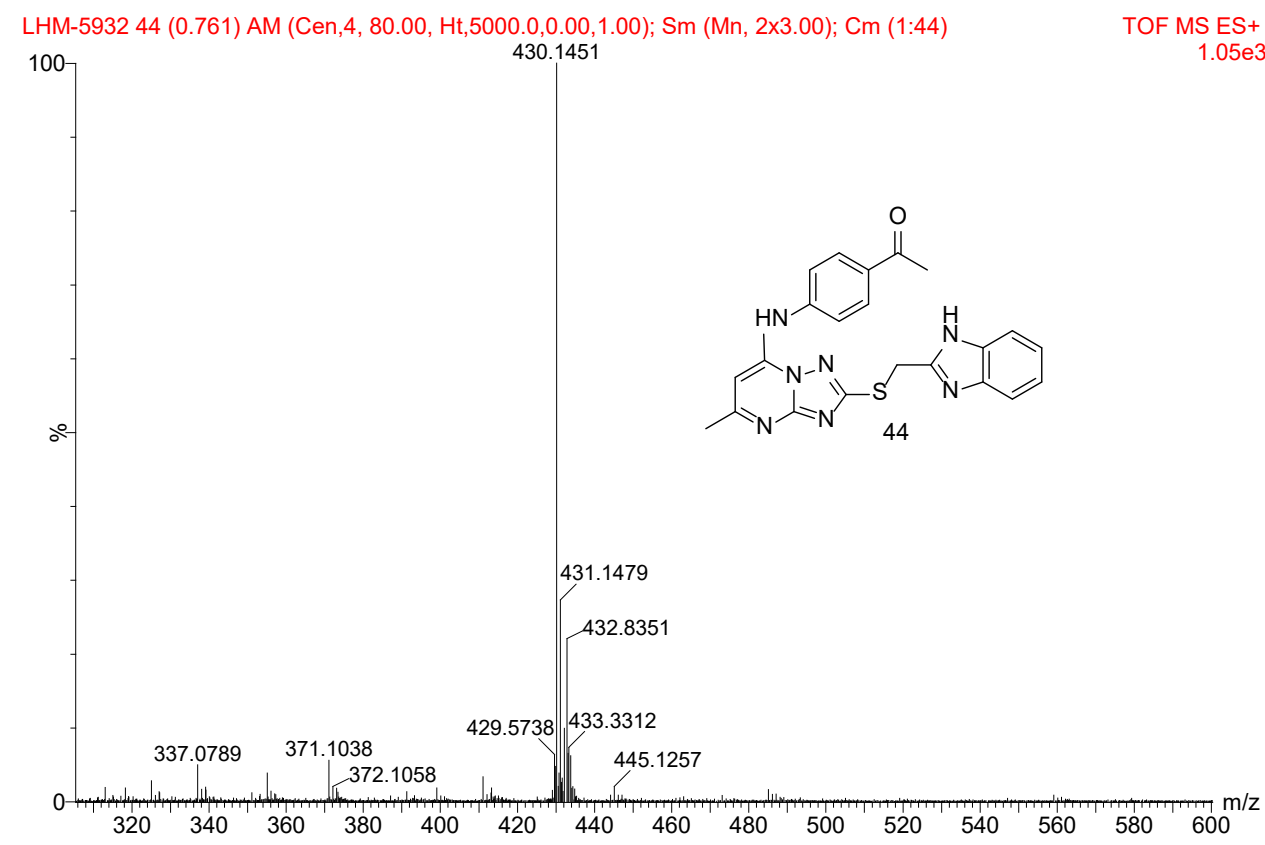


HRMS (ESI): m/z calcd for $\mathrm{C}_{20} \mathrm{H}_{16} \mathrm{Cl}_{2} \mathrm{~N}_{7} \mathrm{~S}(\mathrm{M}+\mathrm{H})^{+}$, 456.0565; found, 456.0564.

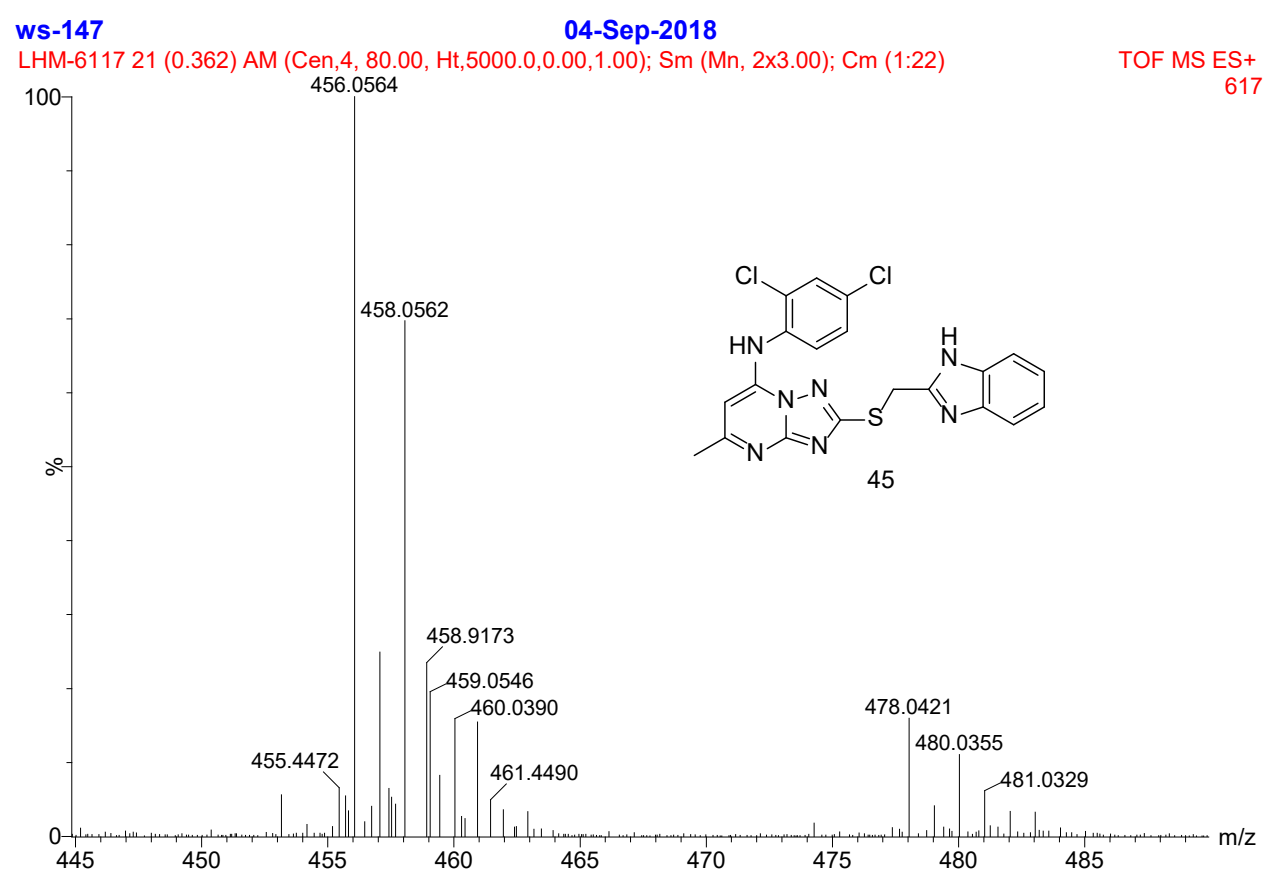

HRMS (ESI): $\mathrm{m} / \mathrm{z}$ calcd for $\mathrm{C}_{21} \mathrm{H}_{18} \mathrm{~N}_{7} \mathrm{~S}(\mathrm{M}-\mathrm{H})^{+}, 400.1344$; found, 400.1352.

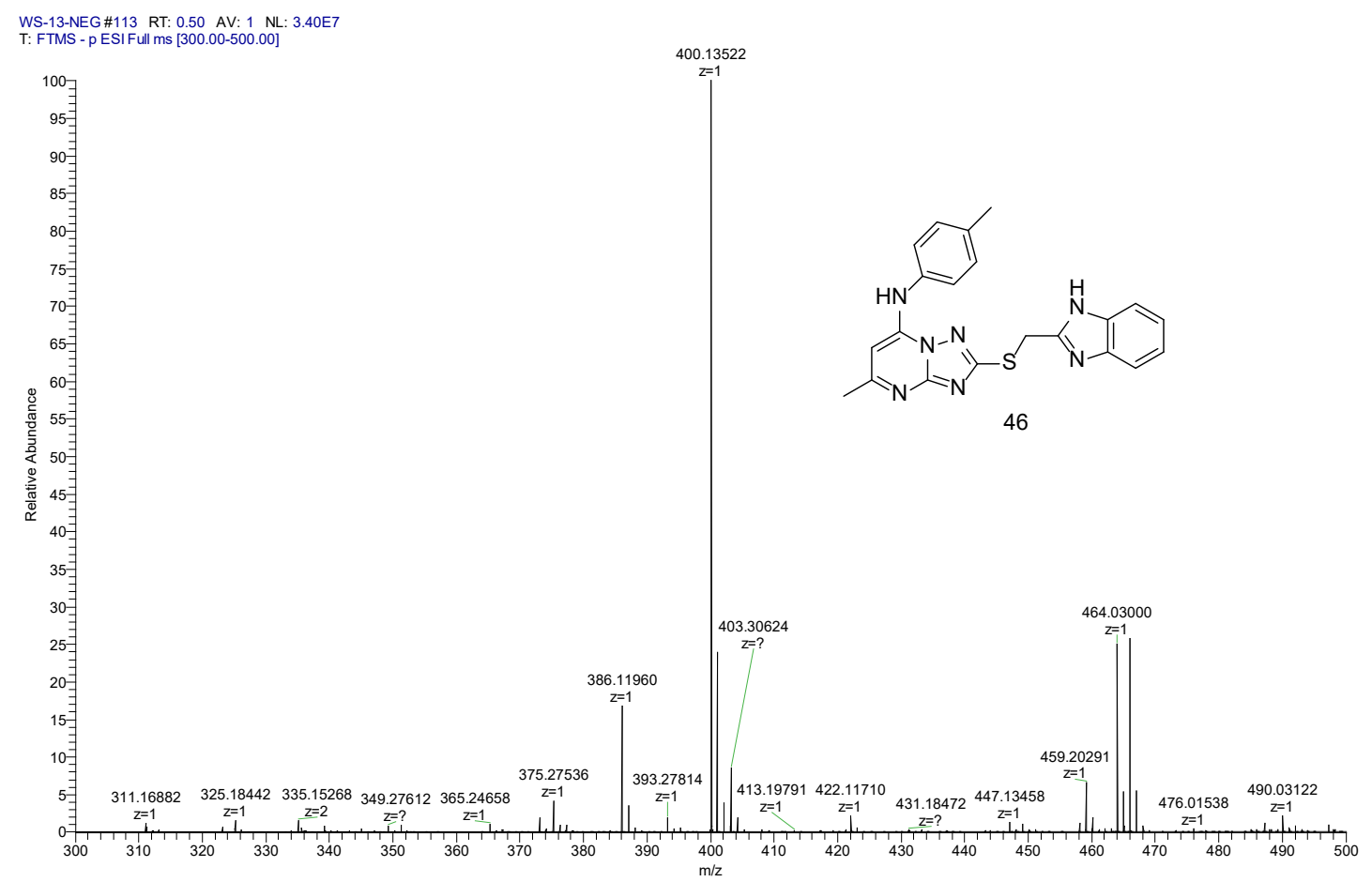


HRMS (ESI): $\mathrm{m} / \mathrm{z}$ calcd for $\mathrm{C}_{21} \mathrm{H}_{20} \mathrm{~N}_{7} \mathrm{OS}(\mathrm{M}+\mathrm{H})^{+}, 418.1450$; found, 418.1451 .

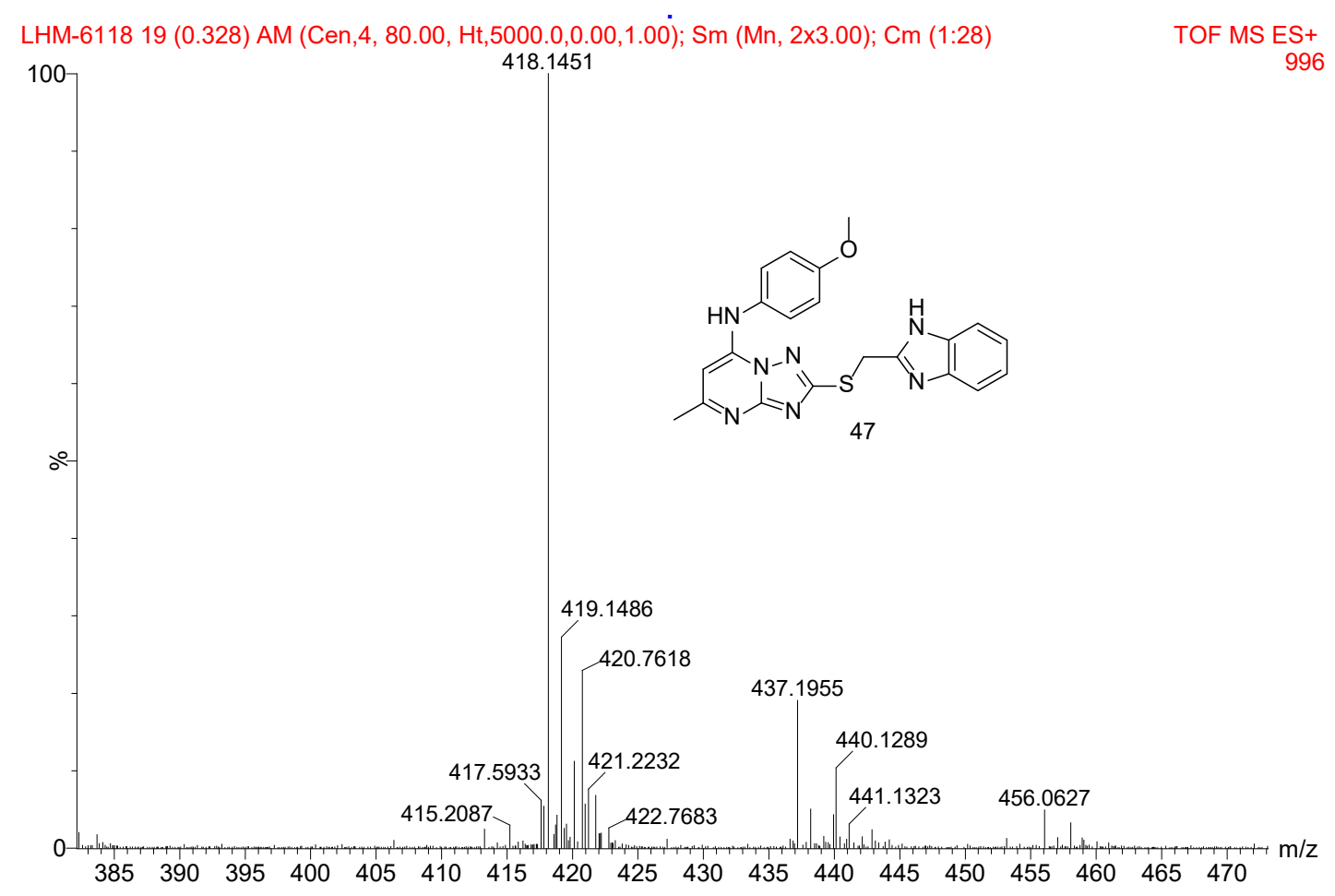

HRMS (ESI): m/z calcd for $\mathrm{C}_{21} \mathrm{H}_{20} \mathrm{~N}_{7} \mathrm{OS}(\mathrm{M}+\mathrm{H})^{+}, 418.1450$; found, 418.1451.

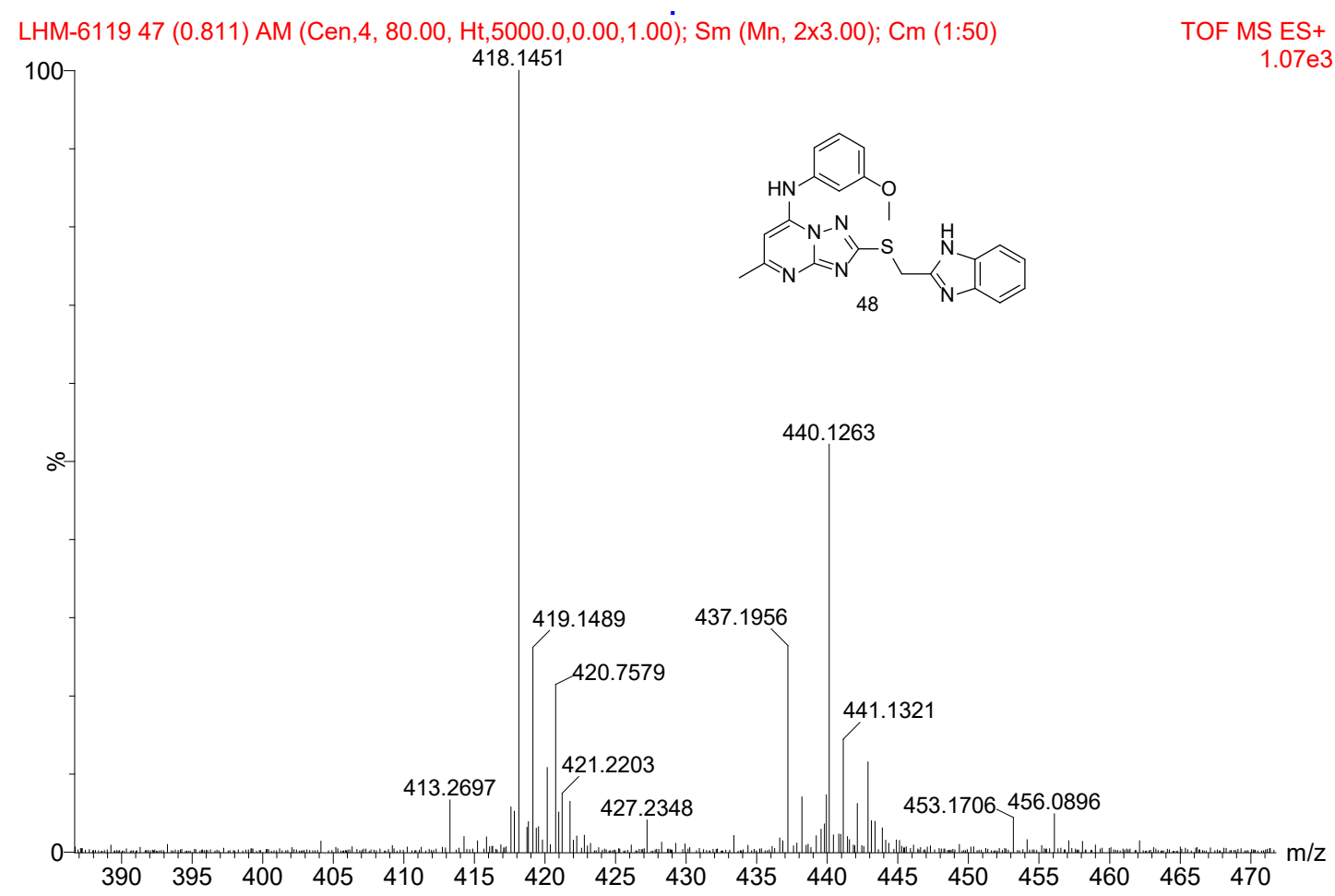


HRMS (ESI): $\mathrm{m} / \mathrm{z}$ calcd for $\mathrm{C}_{23} \mathrm{H}_{22} \mathrm{~N}_{7} \mathrm{O}_{3} \mathrm{~S}(\mathrm{M}-\mathrm{H})^{+}, 476.1505$; found, 476.1513 .

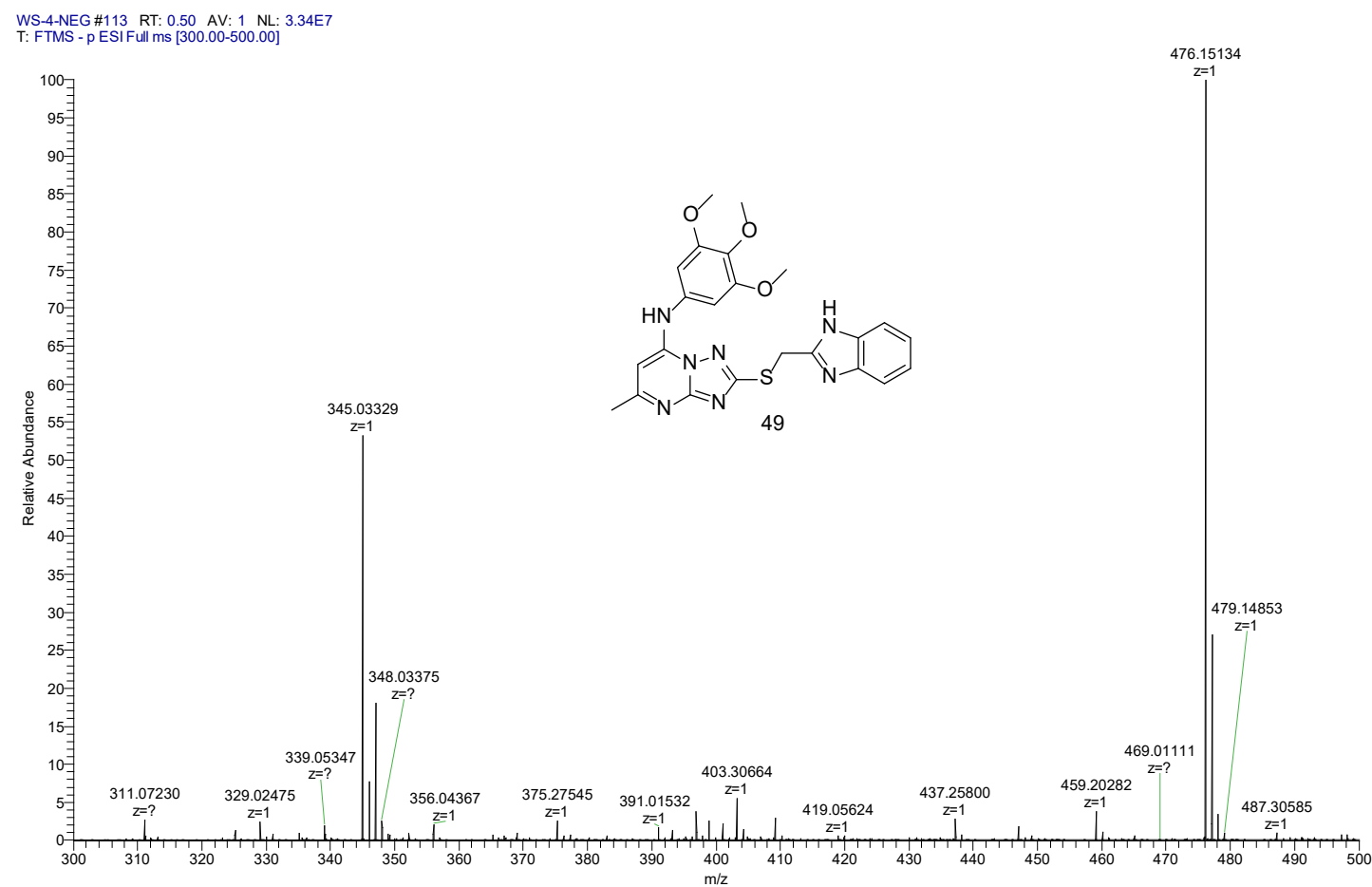

HRMS (ESI): $\mathrm{m} / \mathrm{z}$ calcd for $\mathrm{C}_{20} \mathrm{H}_{18} \mathrm{~N}_{7} \mathrm{OS}(\mathrm{M}+\mathrm{H})^{+}, 404.1294$; found, 404.1293.

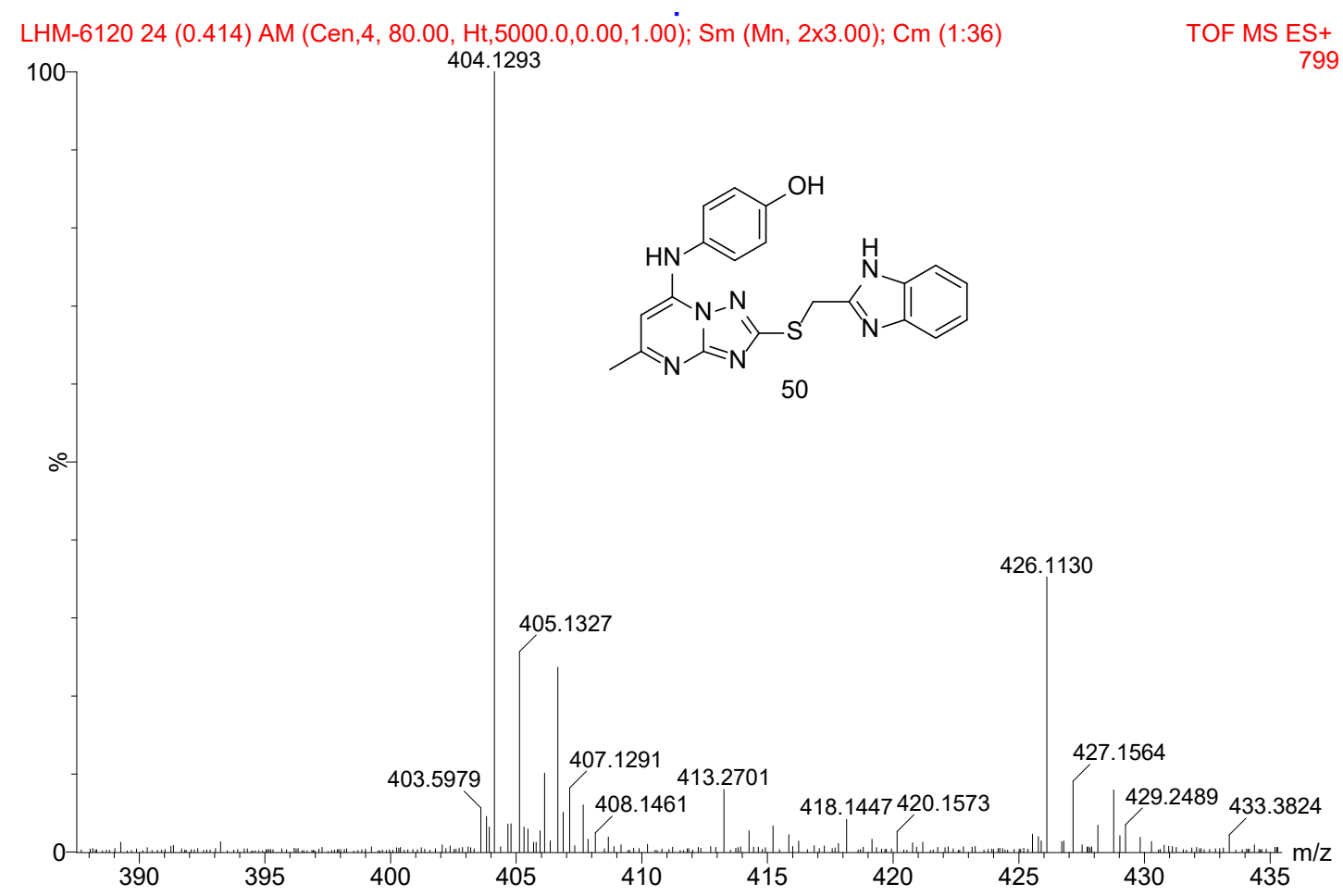


HRMS (ESI): m/z calcd for $\mathrm{C}_{20} \mathrm{H}_{17} \mathrm{ClN}_{7} \mathrm{OS}(\mathrm{M}+\mathrm{H})^{+}$, 438.0904; found, 438.0905.

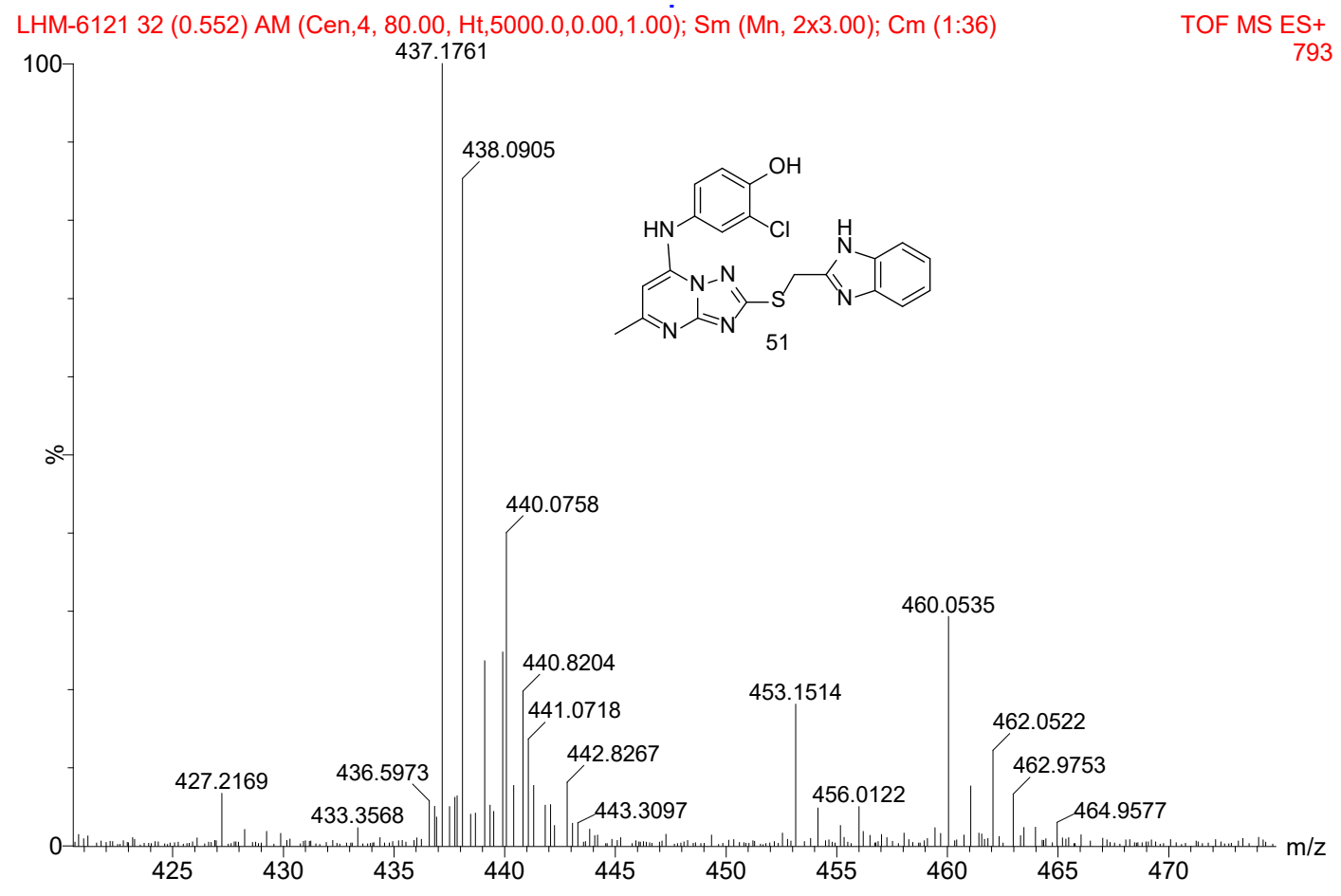

HRMS (ESI): $\mathrm{m} / \mathrm{z}$ calcd for $\mathrm{C}_{22} \mathrm{H}_{18} \mathrm{~N}_{7} \mathrm{~S}(\mathrm{M}+\mathrm{H})^{+}, 412.1344$; found, 412.1345 .

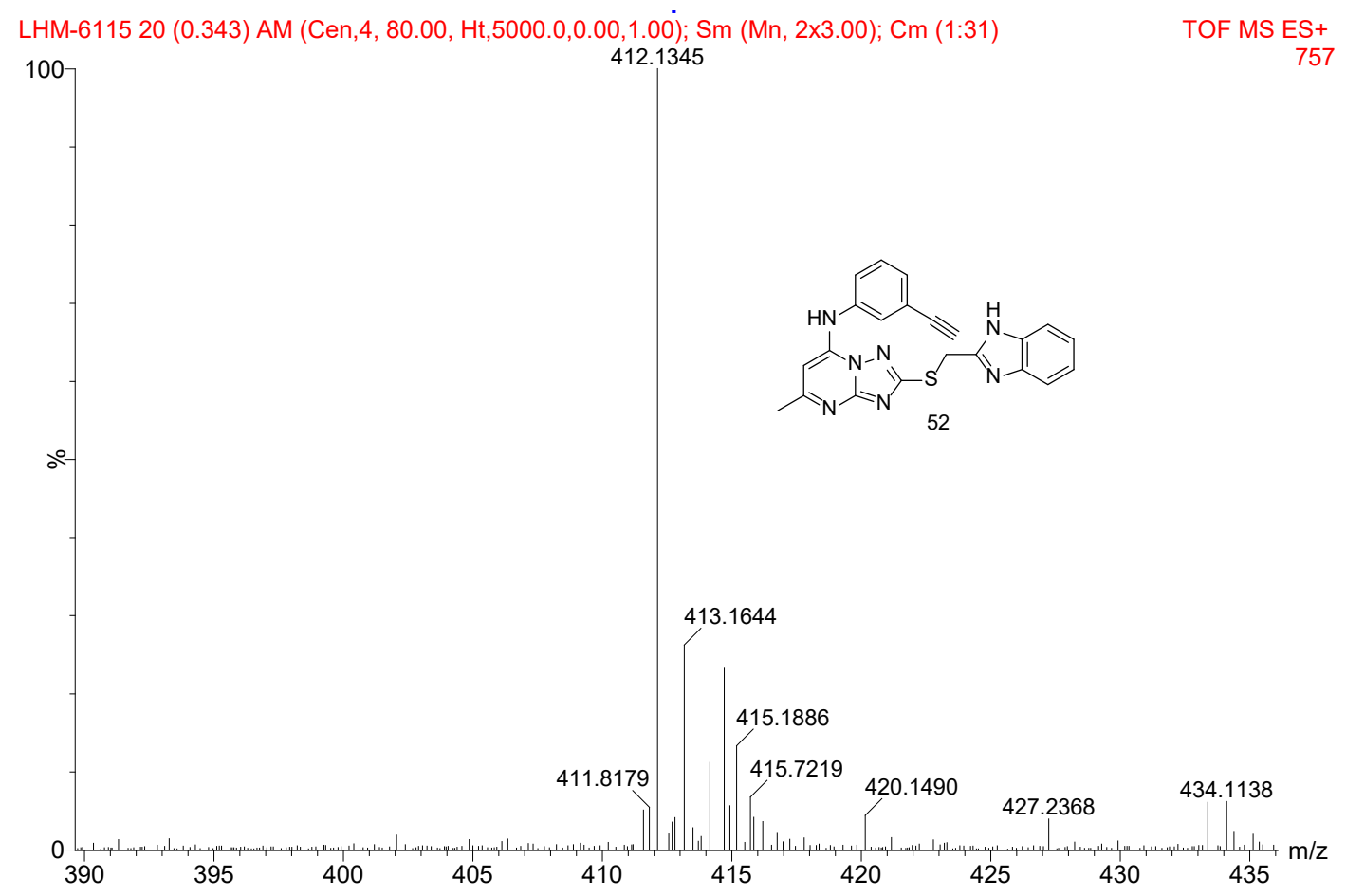


HRMS (ESI): $\mathrm{m} / \mathrm{z}$ calcd for $\mathrm{C}_{25} \mathrm{H}_{28} \mathrm{~N}_{9} \mathrm{~S}(\mathrm{M}+\mathrm{H})^{+}, 486.2188$; found, 486.2148 .

$180 \# 21$ RT: 0.20 AV: 1 NL: $1.15 E 7$
T: FTMS + p ESI Full ms $[100.00-800.00]$

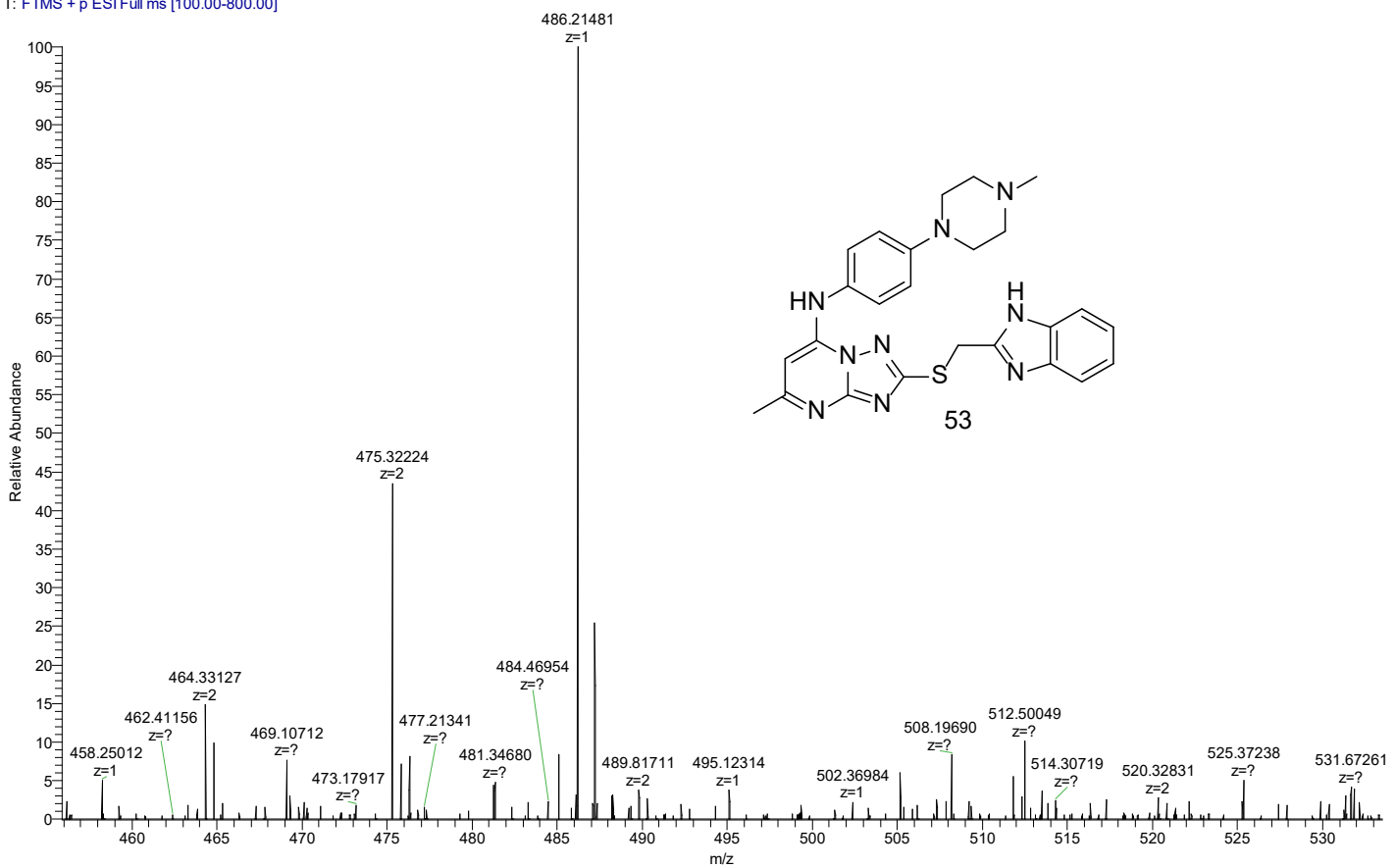

HRMS (ESI): m/z calcd for $\mathrm{C}_{24} \mathrm{H}_{20} \mathrm{~N}_{7} \mathrm{O}_{2} \mathrm{~S}(\mathrm{M}+\mathrm{H})^{+}, 470.1399$; found, 470.1355 .

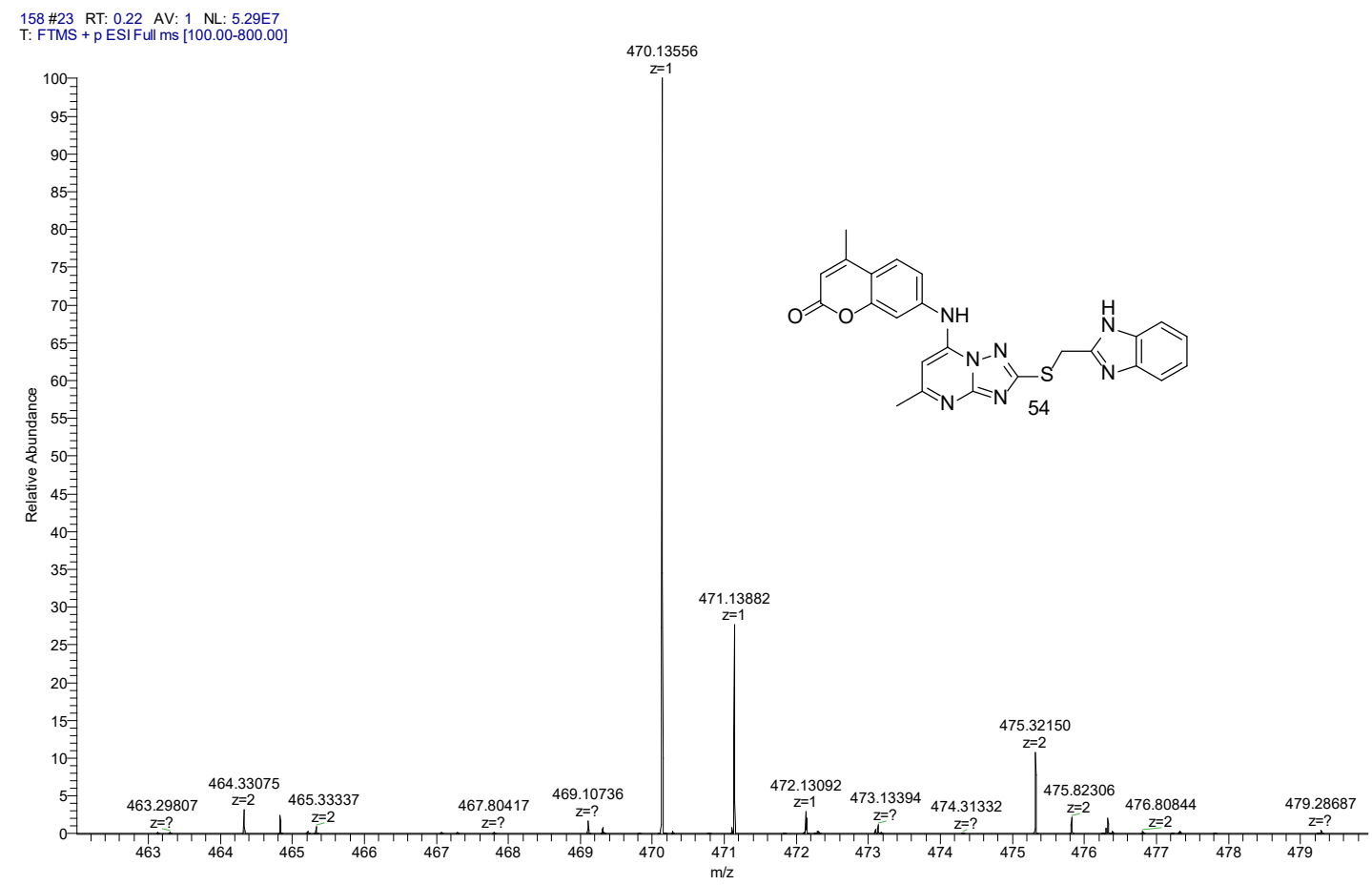


HRMS (ESI): m/z calcd for $\mathrm{C}_{18} \mathrm{H}_{18} \mathrm{~N}_{9} \mathrm{~S}(\mathrm{M}+\mathrm{H})^{+}, 392.1406$; found, 392.1402 .

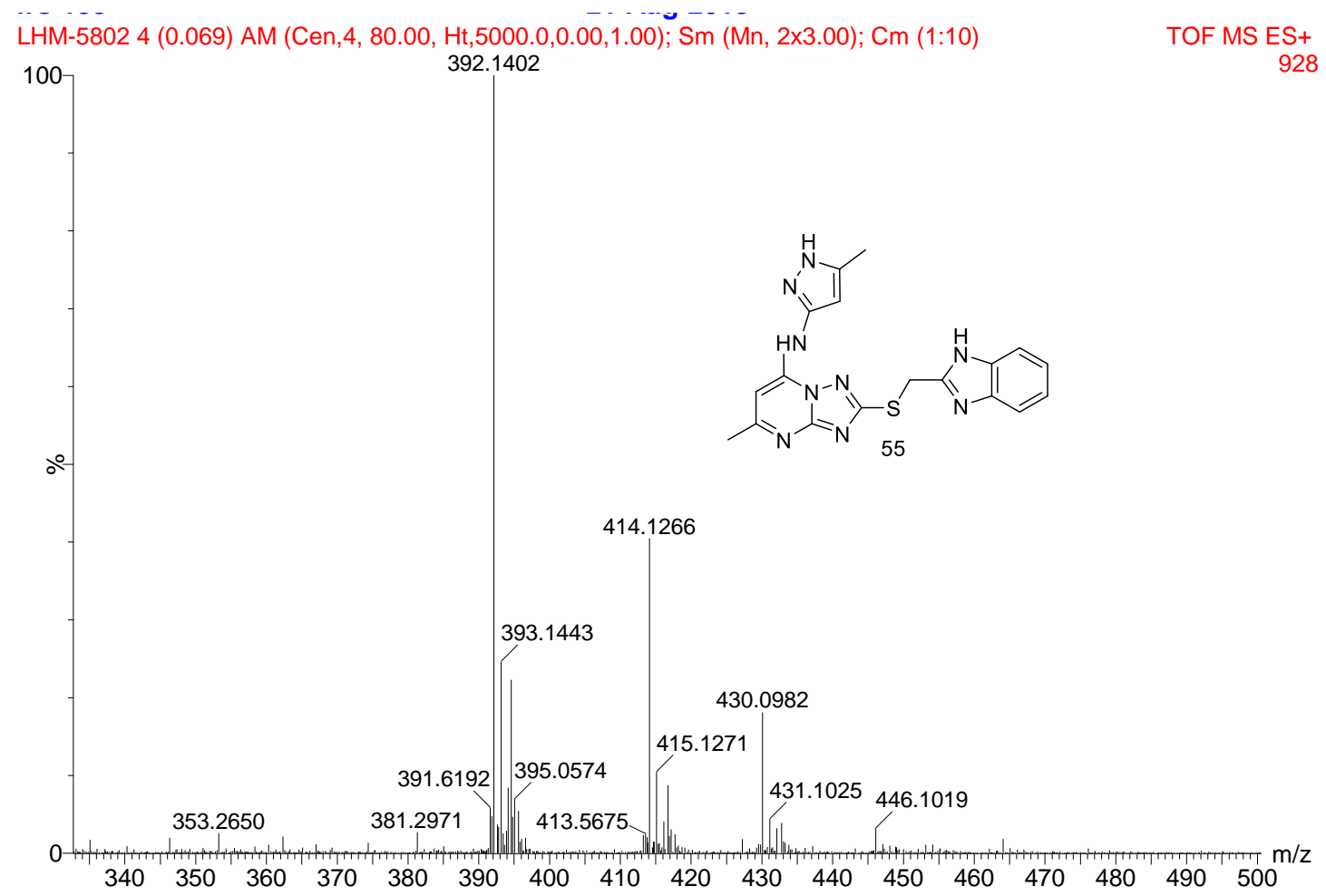




\begin{tabular}{|c|c|c|c|}
\hline Instrument: & Waters e2695 & Column: & $\begin{array}{c}\text { Phenomenex column, C-18, } \\
5.0 \mu \mathrm{m}, 4.6 * 250 \mathrm{~mm}\end{array}$ \\
\hline Mobile Phase: & $\begin{array}{c}\text { Phase A: water; } \\
\text { Phase B: acetonitrile; } \\
\text { A/ B = 60/40, v/v }\end{array}$ & Flow rate: & $1.0 \mathrm{~mL} / \mathrm{min}$ \\
\hline Ret. Time (min): & 4.751 & Purity $(\%)$ & 97.80 \\
\hline
\end{tabular}

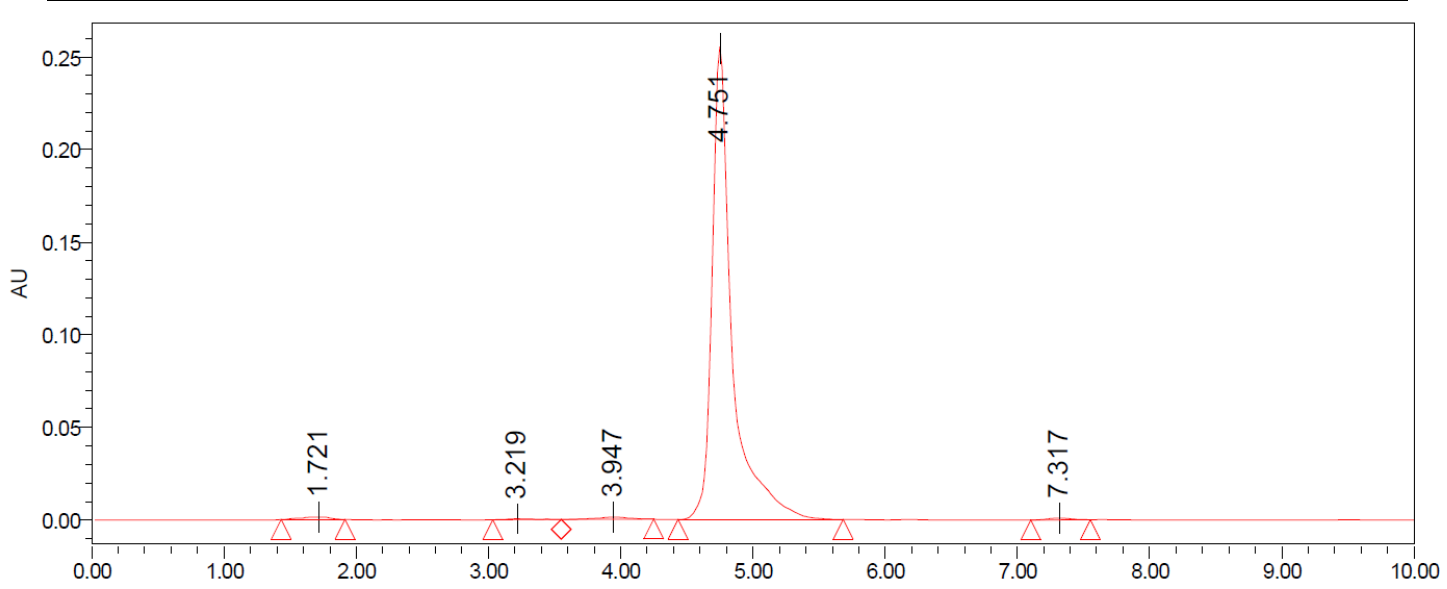

Figure S1. HPLC spectrum of compound $\mathbf{5}$

\begin{tabular}{|c|c|c|c|}
\hline Instrument: & Waters e2695 & Column: & $\begin{array}{c}\text { Phenomen column, C-18,5.0 } \mu \mathrm{m}, \\
4.6 * 250 \mathrm{~mm}\end{array}$ \\
\hline Mobile Phase: & $\begin{array}{c}\text { Phase A: water; } \\
\text { Phase B: acetonitrile; } \\
\text { A/ B = 60/40, v/v }\end{array}$ & $\begin{array}{c}\text { Flow } \\
\text { rate: }\end{array}$ & $1.0 \mathrm{~mL} / \mathrm{min}$ \\
\hline $\begin{array}{c}\text { Ret. Time } \\
\text { (min): }\end{array}$ & 4.785 & $\begin{array}{c}\text { Purity } \\
(\%)\end{array}$ & 99.02 \\
\hline
\end{tabular}

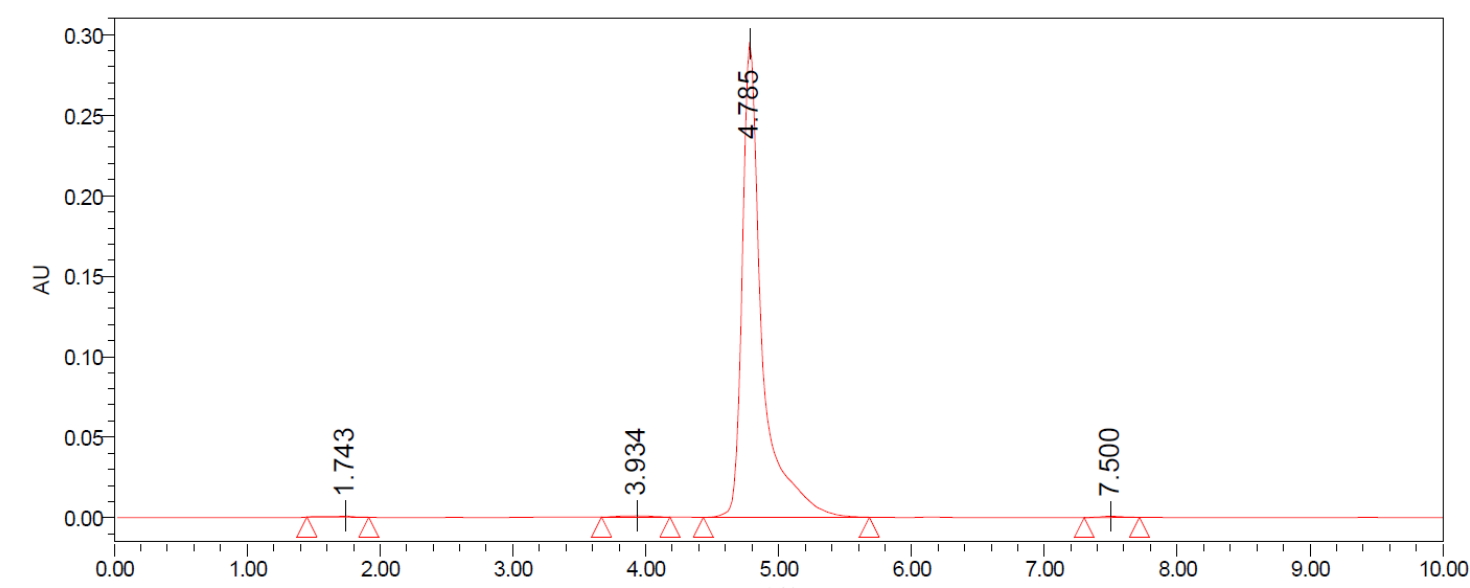

Figure S2. HPLC spectrum of compound 6 


\begin{tabular}{|c|c|c|c|}
\hline Instrument: & Waters e2695 & Column: & $\begin{array}{c}\text { Phenomenex column, C-18, } \\
5.0 \mu \mathrm{m}, 4.6 * 250 \mathrm{~mm}\end{array}$ \\
\hline Mobile Phase: & $\begin{array}{c}\text { Phase A: water; } \\
\text { Phase B: acetonitrile; } \\
\text { A/ B = 60/40, v/v }\end{array}$ & Flow rate: & $1.0 \mathrm{~mL} / \mathrm{min}$ \\
\hline $\begin{array}{c}\text { Ret. Time } \\
\text { (min): }\end{array}$ & 4.778 & Purity (\%) & 99.56 \\
\hline
\end{tabular}

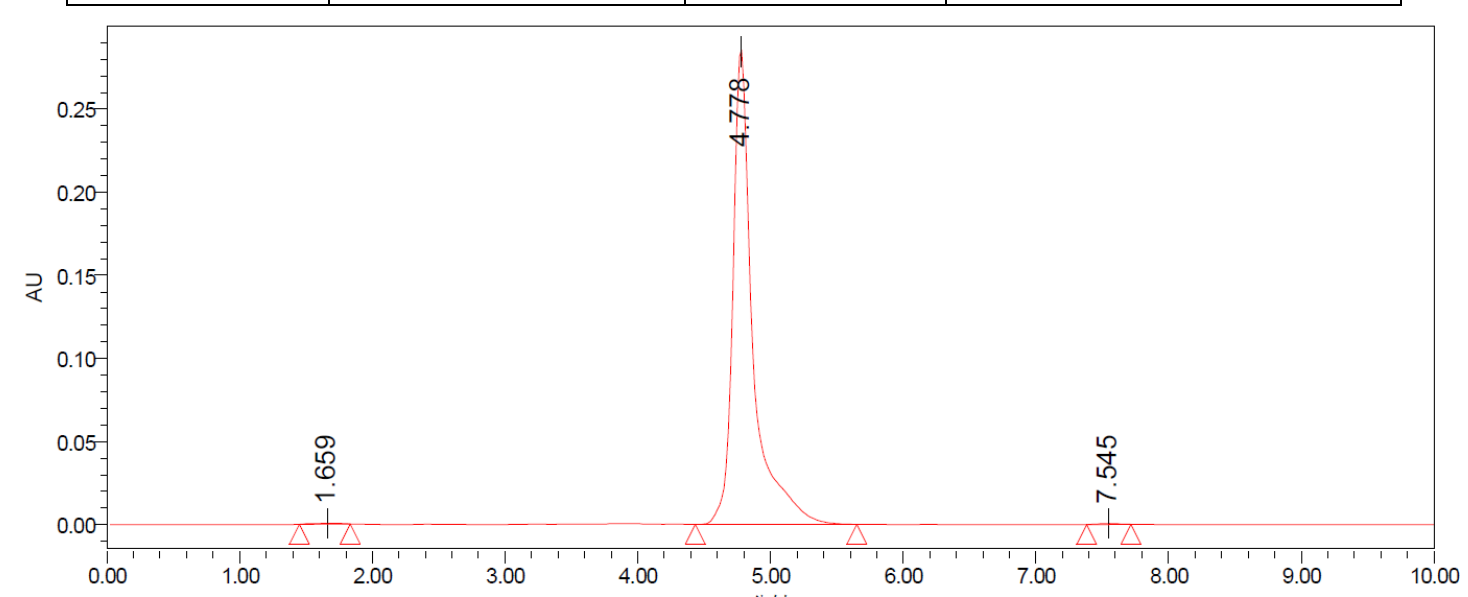

Figure S3. HPLC spectrum of compound 7

\begin{tabular}{|c|c|c|c|}
\hline Instrument: & Waters e2695 & Column: & $\begin{array}{c}\text { Phenomenex column, C-18, 5.0 } \\
\mu \mathrm{m}, 4.6 * 250 \mathrm{~mm}\end{array}$ \\
\hline Mobile Phase: & $\begin{array}{c}\text { Phase A: water; } \\
\text { Phase B: acetonitrile; } \\
\text { A/ B = 60/40, v/v }\end{array}$ & Flow rate: & $1.0 \mathrm{~mL} / \mathrm{min}$ \\
\hline Ret. Time (min): & 5.143 & Purity (\%) & 96.35 \\
\hline
\end{tabular}

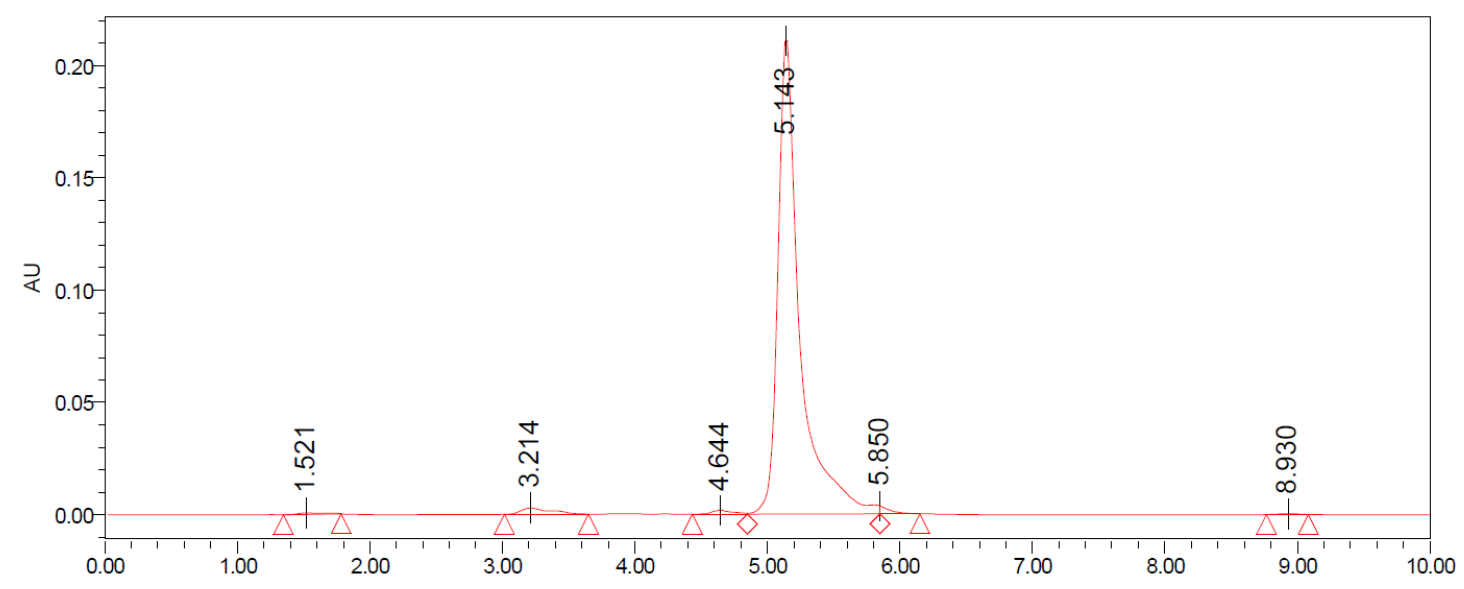

Figure S4. HPLC spectrum of compound $\mathbf{8}$ 


\begin{tabular}{|c|c|c|c|}
\hline Instrument: & Waters e2695 & Column: & $\begin{array}{c}\text { Phenomenex column, C-18, 5.0 } \\
\mu \mathrm{m}, 4.6 * 250 \mathrm{~mm}\end{array}$ \\
\hline Mobile Phase: & $\begin{array}{c}\text { Phase A: water; } \\
\text { Phase B: acetonitrile; } \\
\text { A/ B }=60 / 40, \mathrm{v} / \mathrm{v}\end{array}$ & Flow rate: & $1.0 \mathrm{~mL} / \mathrm{min}$ \\
\hline Ret. Time (min): & 5.028 & Purity $(\%)$ & 98.94 \\
\hline
\end{tabular}

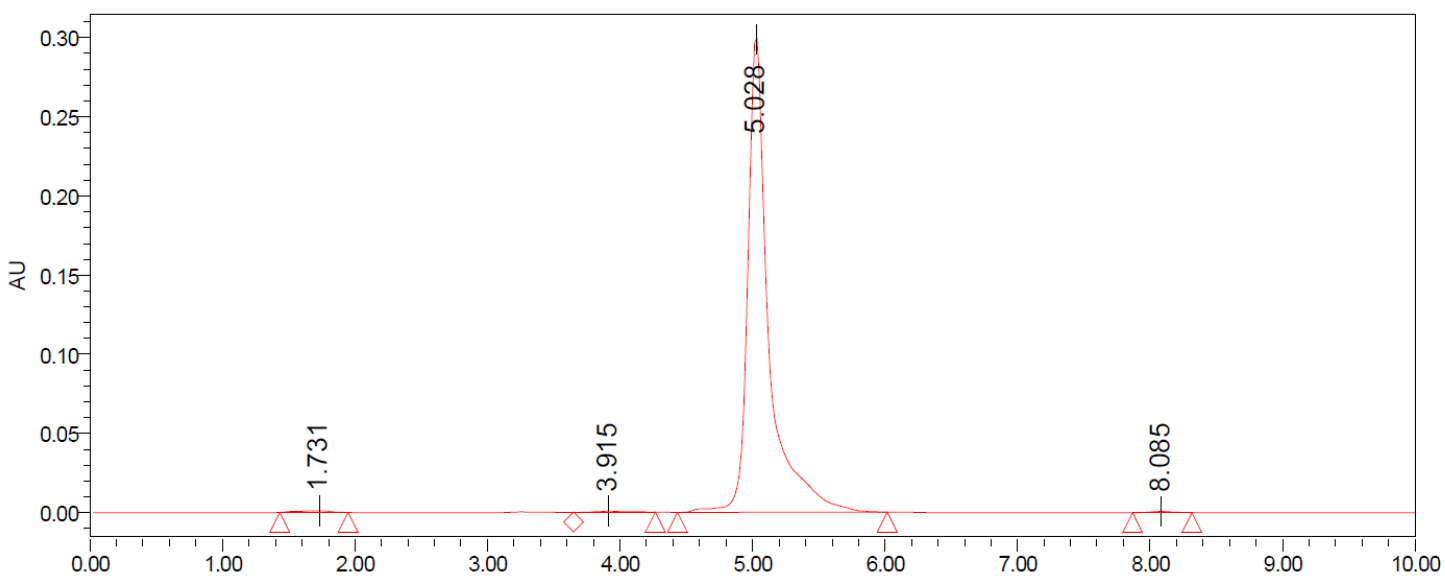

Figure S5. HPLC spectrum of compound 9

\begin{tabular}{|c|c|c|c|}
\hline Instrument: & Waters e2695 & Column: & $\begin{array}{c}\text { Phenomenex column, C-18, } \\
5.0 \mu \mathrm{m}, 4.6 * 250 \mathrm{~mm}\end{array}$ \\
\hline Mobile Phase: & $\begin{array}{c}\text { Phase A: water; } \\
\text { Phase B: acetonitrile; } \\
\text { A/ B }=60 / 40, \mathrm{v} / \mathrm{v}\end{array}$ & Flow rate: & $1.0 \mathrm{~mL} / \mathrm{min}$ \\
\hline Ret. Time (min): & 4.743 & Purity (\%) & 97.76 \\
\hline
\end{tabular}

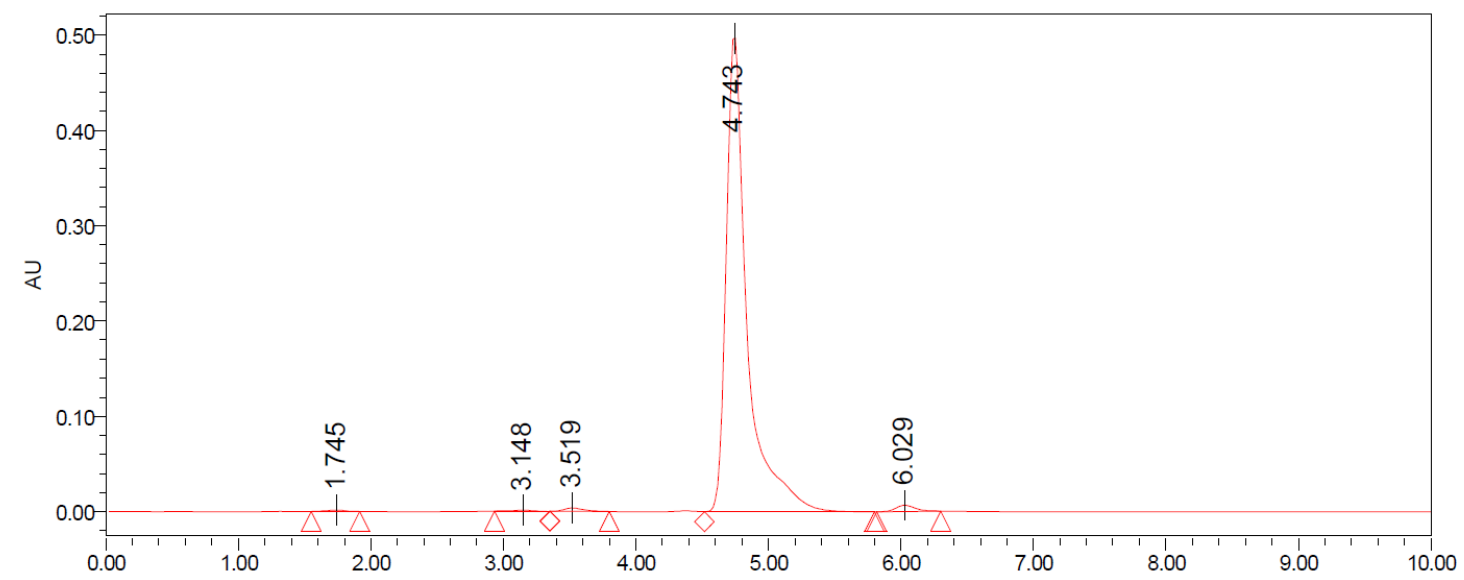

Figure S6. HPLC spectrum of compound 10 


\begin{tabular}{|c|c|c|c|}
\hline Instrument: & Waters e2695 & Column: & $\begin{array}{c}\text { Phenomenex column, C-18, 5.0 } \\
\mu \mathrm{m}, 4.6 * 250 \mathrm{~mm}\end{array}$ \\
\hline Mobile Phase: & $\begin{array}{c}\text { Phase A: water; } \\
\text { Phase B: acetonitrile; } \\
\text { A/ B = 60/40, v/v }\end{array}$ & Flow rate: & $1.0 \mathrm{~mL} / \mathrm{min}$ \\
\hline Ret. Time (min): & 4.409 & Purity (\%) & 99.94 \\
\hline
\end{tabular}

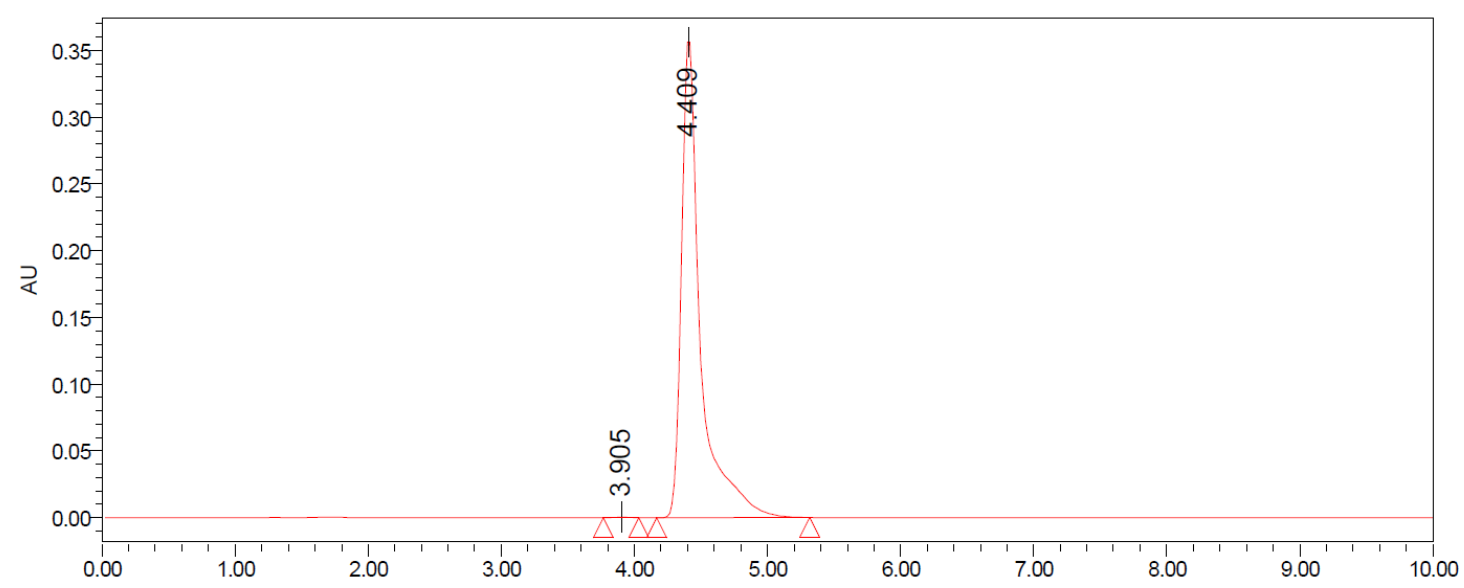

Figure S7. HPLC spectrum of compound 11

\begin{tabular}{|c|c|c|c|}
\hline Instrument: & Waters e2695 & Column: & $\begin{array}{c}\text { Phenomenex column, C-18, 5.0 } \\
\mu \mathrm{m}, 4.6 * 250 \mathrm{~mm}\end{array}$ \\
\hline Mobile Phase: & $\begin{array}{c}\text { Phase A: water; } \\
\text { Phase B: acetonitrile; } \\
\text { A/ B = 60/40, v/v }\end{array}$ & Flow rate: & $1.0 \mathrm{~mL} / \mathrm{min}$ \\
\hline Ret. Time (min): & 3.820 & Purity (\%) & 99.08 \\
\hline
\end{tabular}

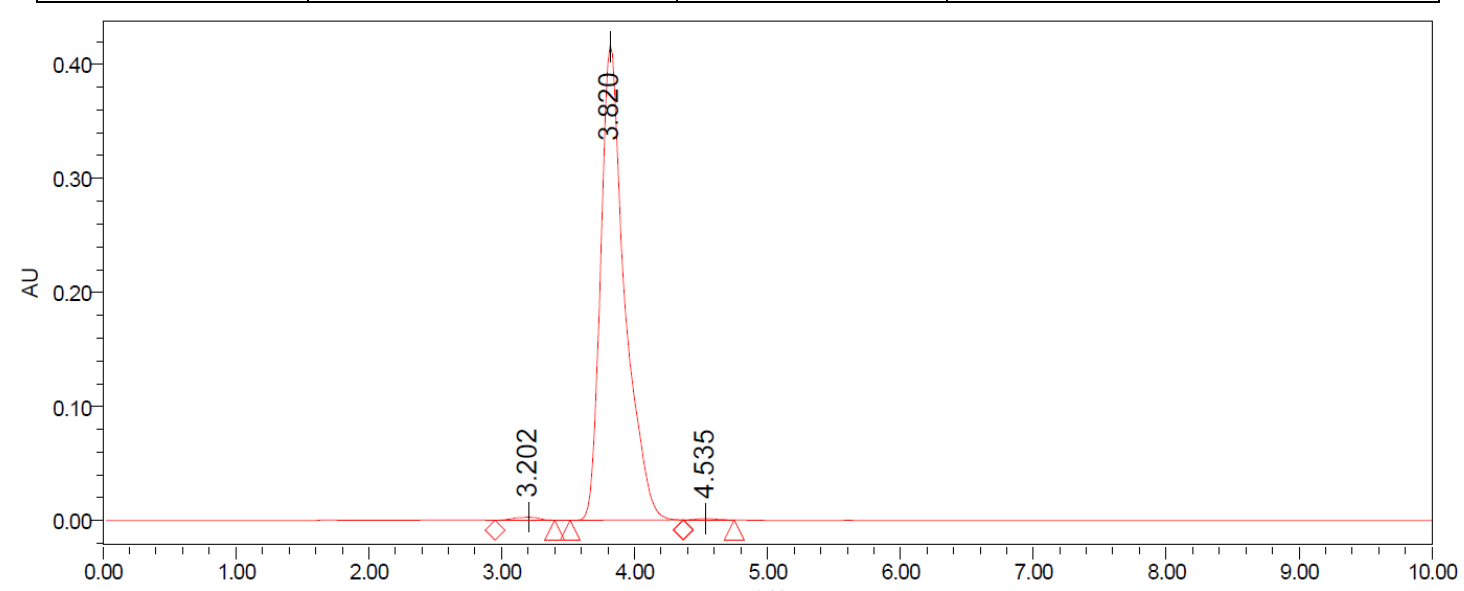

Figure S8. HPLC spectrum of compound 12 


\begin{tabular}{|c|c|c|c|}
\hline Instrument: & Waters e2695 & Column: & $\begin{array}{c}\text { Phenomenex column, C-18, } \\
5.0 \mu \mathrm{m}, 4.6 * 250 \mathrm{~mm}\end{array}$ \\
\hline Mobile Phase: & $\begin{array}{c}\text { Phase A: water; } \\
\text { Phase B: acetonitrile; } \\
\text { A/ B =60/40, v/v }\end{array}$ & Flow rate: & $1.0 \mathrm{~mL} / \mathrm{min}$ \\
\hline Ret. Time (min): & 3.921 & Purity (\%) & 99.85 \\
\hline
\end{tabular}

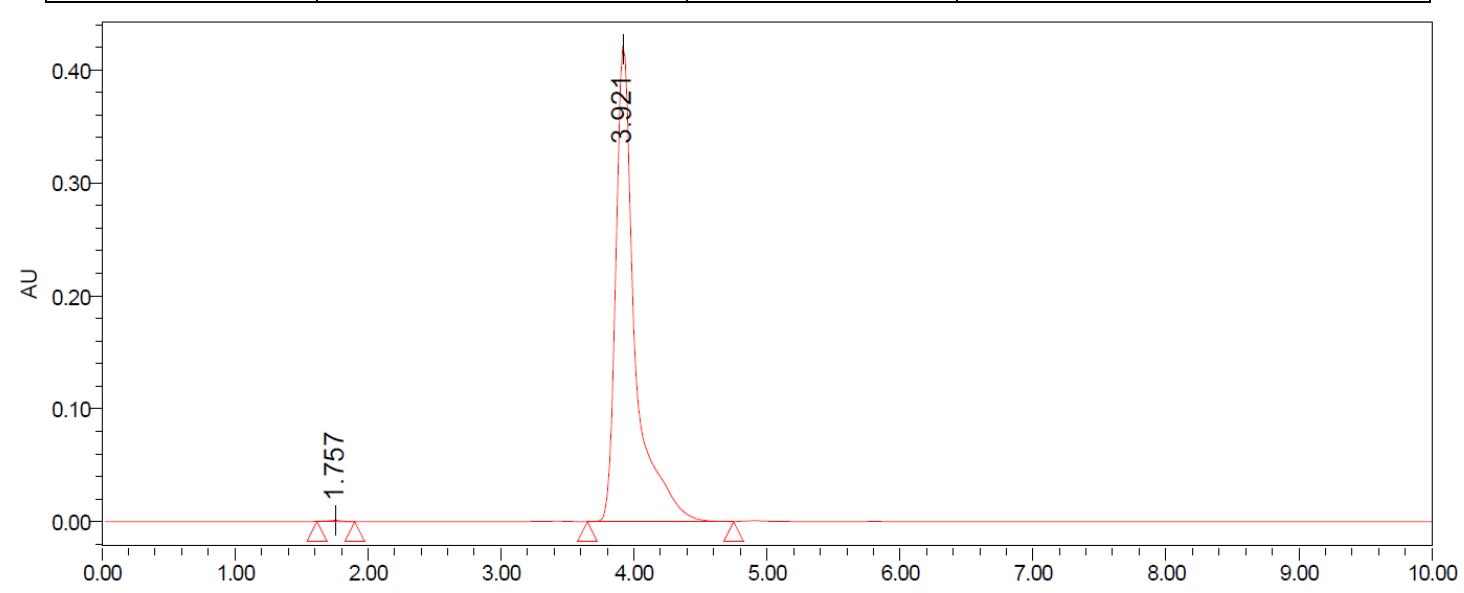

Figure S9. HPLC spectrum of compound $\mathbf{1 3}$

\begin{tabular}{|c|c|c|c|}
\hline Instrument: & Waters e2695 & Column: & $\begin{array}{c}\text { Phenomenex column, C-18, } \\
5.0 \mu \mathrm{m}, 4.6 * 250 \mathrm{~mm}\end{array}$ \\
\hline Mobile Phase: & $\begin{array}{c}\text { Phase A: water; } \\
\text { Phase B: acetonitrile; } \\
\text { A/ B }=60 / 40, \mathrm{v} / \mathrm{v}\end{array}$ & Flow rate: & $1.0 \mathrm{~mL} / \mathrm{min}$ \\
\hline Ret. Time (min): & 1.872 & Purity $(\%)$ & 98.26 \\
\hline
\end{tabular}

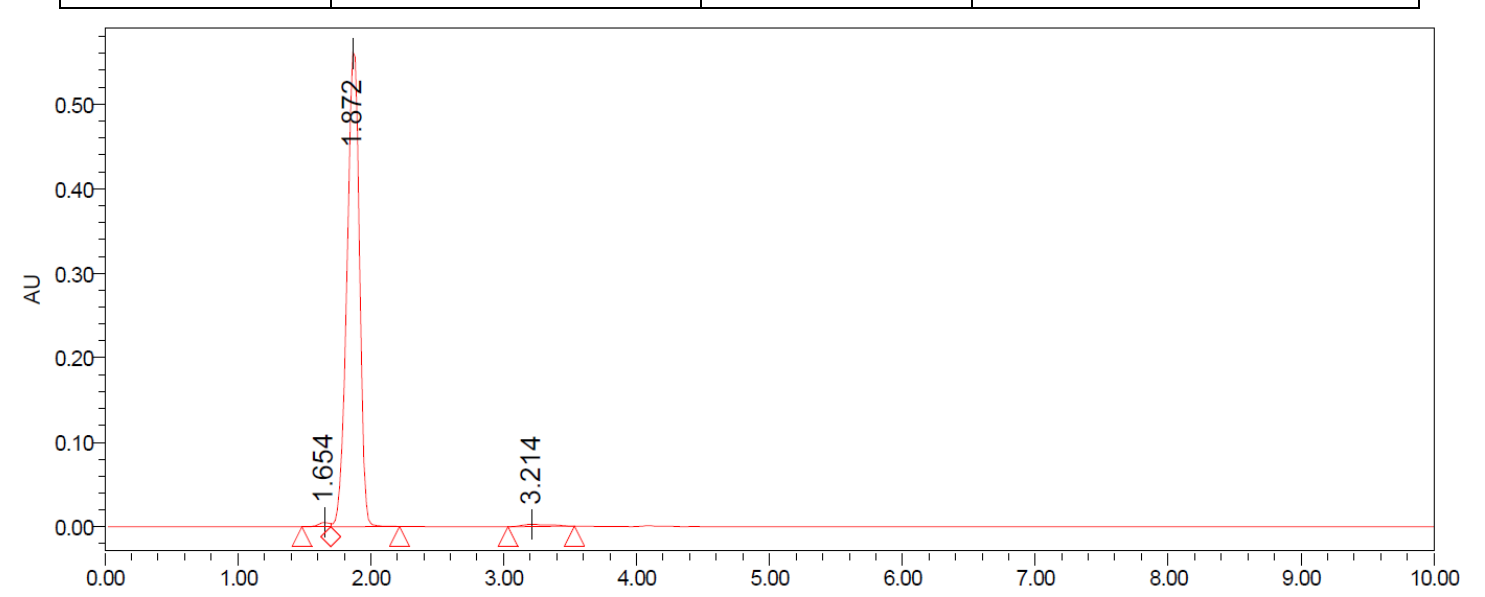

Figure S10. HPLC spectrum of compound 14 


\begin{tabular}{|c|c|c|c|}
\hline Instrument: & Waters e2695 & Column: & $\begin{array}{c}\text { Phenomenex column, C-18, } \\
5.0 \mu \mathrm{m}, 4.6 * 250 \mathrm{~mm}\end{array}$ \\
\hline Mobile Phase: & $\begin{array}{c}\text { Phase A: water; } \\
\text { Phase B: acetonitrile; } \\
\mathrm{A} / \mathrm{B}=60 / 40, \mathrm{v} / \mathrm{v}\end{array}$ & Flow rate: & $1.0 \mathrm{~mL} / \mathrm{min}$ \\
\hline Ret. Time (min): & 4.795 & Purity $(\%)$ & 99.28 \\
\hline
\end{tabular}

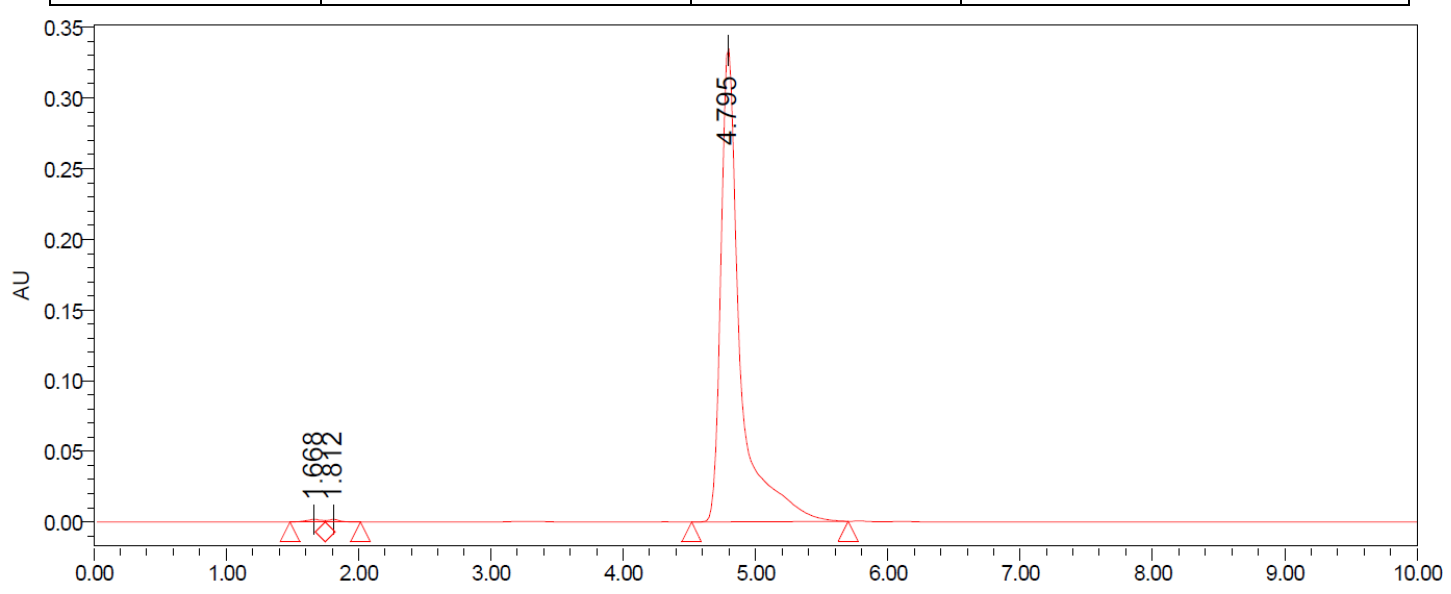

Figure S11. HPLC spectrum of compound $\mathbf{1 5}$

\begin{tabular}{|c|c|c|c|}
\hline Instrument: & Waters e2695 & Column: & $\begin{array}{c}\text { Phenomenex column, C-18, } \\
5.0 \mu \mathrm{m}, 4.6 * 250 \mathrm{~mm}\end{array}$ \\
\hline Mobile Phase: & $\begin{array}{c}\text { Phase A: water; } \\
\text { Phase B: acetonitrile; } \\
\mathrm{A} / \mathrm{B}=60 / 40, \mathrm{v} / \mathrm{v}\end{array}$ & Flow rate: & $1.0 \mathrm{~mL} / \mathrm{min}$ \\
\hline Ret. Time (min): & 3.935 & Purity (\%) & 99.28 \\
\hline
\end{tabular}

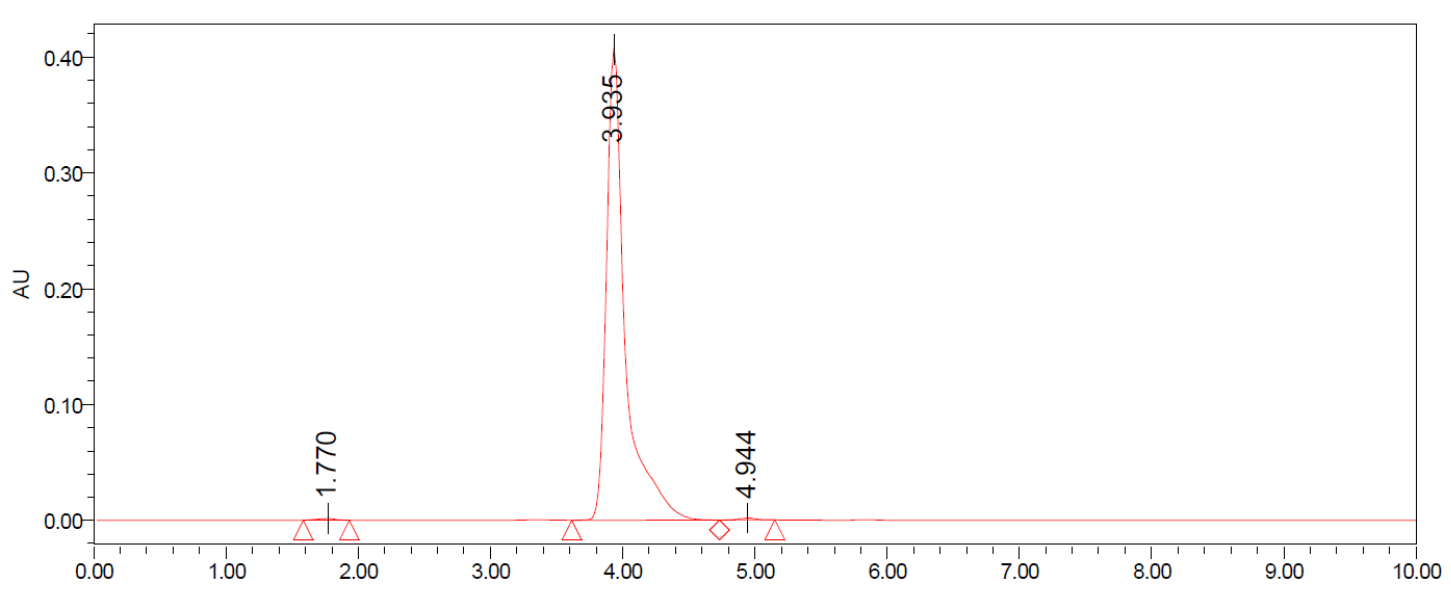

Figure S12. HPLC spectrum of compound $\mathbf{1 6}$ 


\begin{tabular}{|c|c|c|c|}
\hline Instrument: & Waters e2695 & Column: & $\begin{array}{c}\text { Phenomenex column, C-18, } \\
5.0 \mu \mathrm{m}, 4.6 * 250 \mathrm{~mm}\end{array}$ \\
\hline Mobile Phase: & $\begin{array}{c}\text { Phase A: water; } \\
\text { Phase B: acetonitrile; } \\
\text { A/ B = 60/40, v/v }\end{array}$ & Flow rate: & $1.0 \mathrm{~mL} / \mathrm{min}$ \\
\hline Ret. Time (min): & 3.735 & Purity (\%) & 98.85 \\
\hline
\end{tabular}

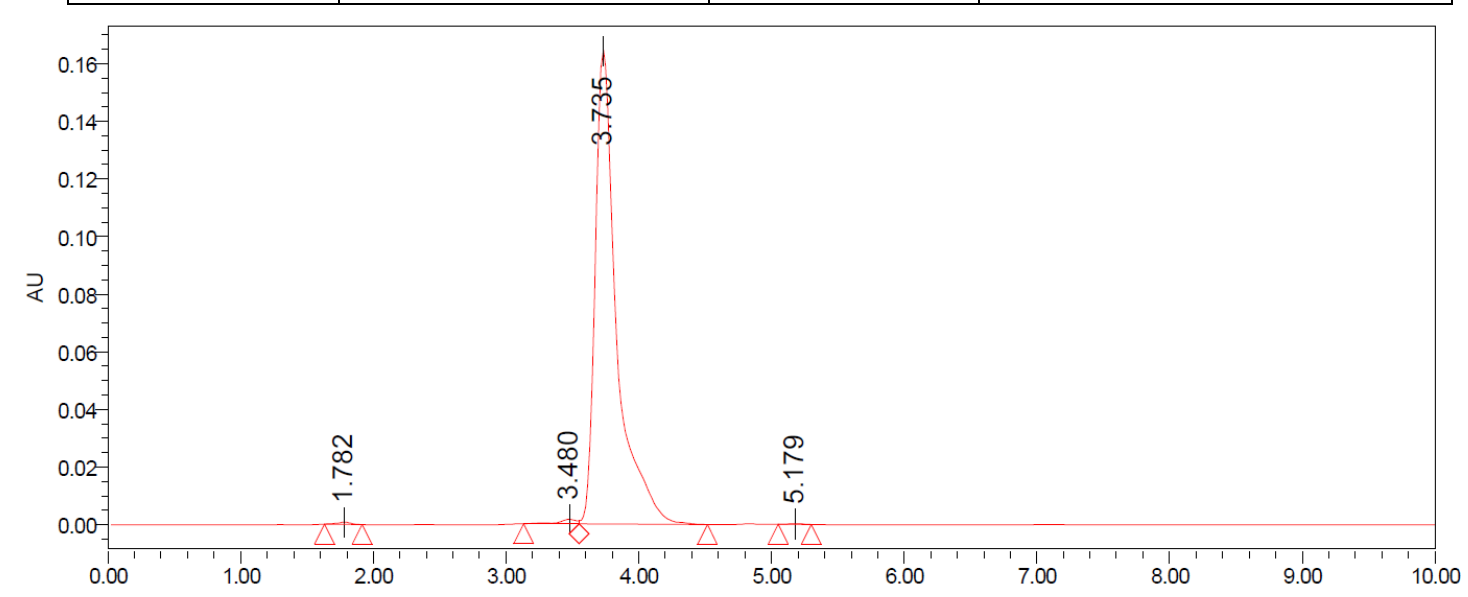

Figure S13. HPLC spectrum of compound $\mathbf{1 7}$

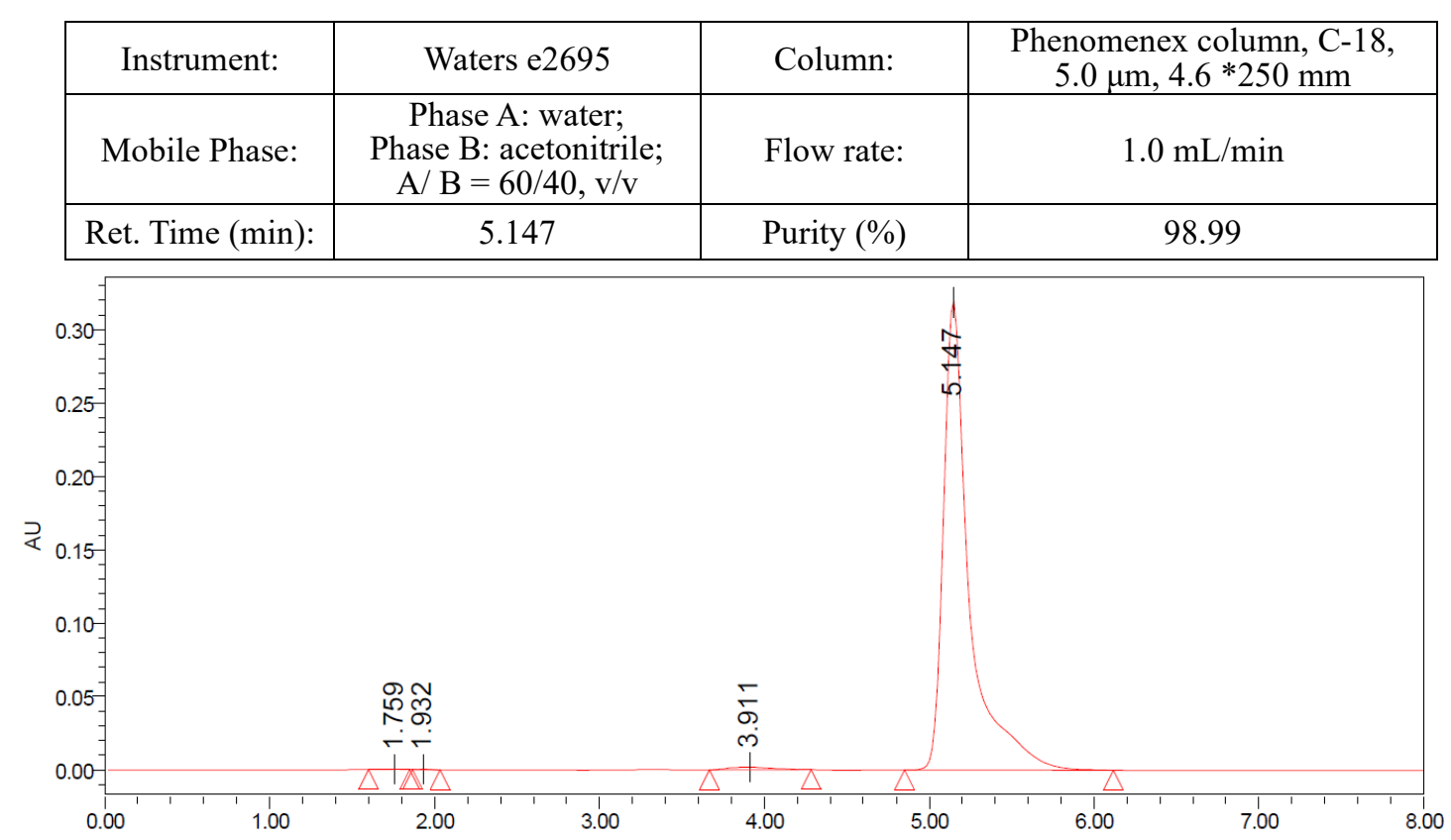

Figure S14. HPLC spectrum of compound $\mathbf{1 8}$ 


\begin{tabular}{|c|c|c|c|}
\hline Instrument: & Waters e2695 & Column: & $\begin{array}{c}\text { Phenomenex column, C-18, } \\
5.0 \mu \mathrm{m}, 4.6 * 250 \mathrm{~mm}\end{array}$ \\
\hline Mobile Phase: & $\begin{array}{c}\text { Phase A: water; } \\
\text { Phase B: acetonitrile; } \\
\mathrm{A} / \mathrm{B}=60 / 40, \mathrm{v} / \mathrm{v}\end{array}$ & Flow rate: & $1.0 \mathrm{~mL} / \mathrm{min}$ \\
\hline Ret. Time (min): & 4.858 & Purity (\%) & 96.78 \\
\hline
\end{tabular}

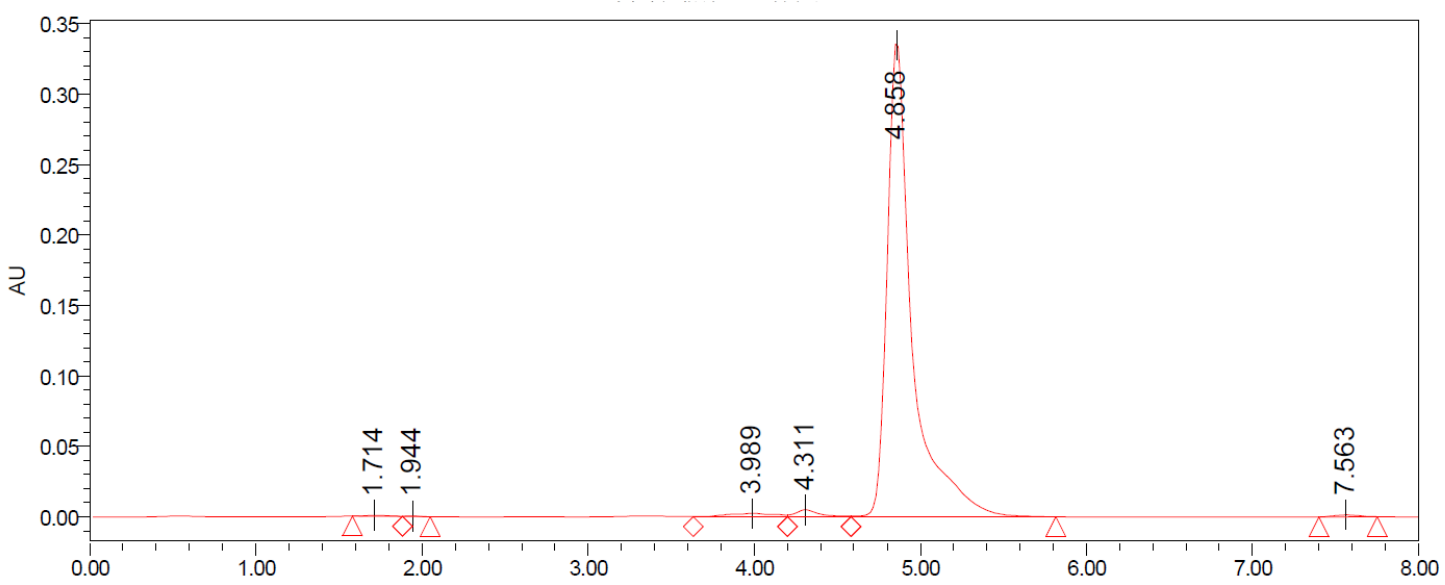

Figure S15. HPLC spectrum of compound 19

\begin{tabular}{|c|c|c|c|}
\hline Instrument: & Waters e2695 & Column: & $\begin{array}{c}\text { Phenomenex column, C-18, } \\
5.0 \mu \mathrm{m}, 4.6 * 250 \mathrm{~mm}\end{array}$ \\
\hline Mobile Phase: & $\begin{array}{c}\text { Phase A: water; } \\
\text { Phase B: acetonitrile; } \\
\text { A/ B }=60 / 40, \mathrm{v} / \mathrm{v}\end{array}$ & Flow rate: & $1.0 \mathrm{~mL} / \mathrm{min}$ \\
\hline Ret. Time (min): & 4.248 & Purity (\%) & 99.22 \\
\hline
\end{tabular}

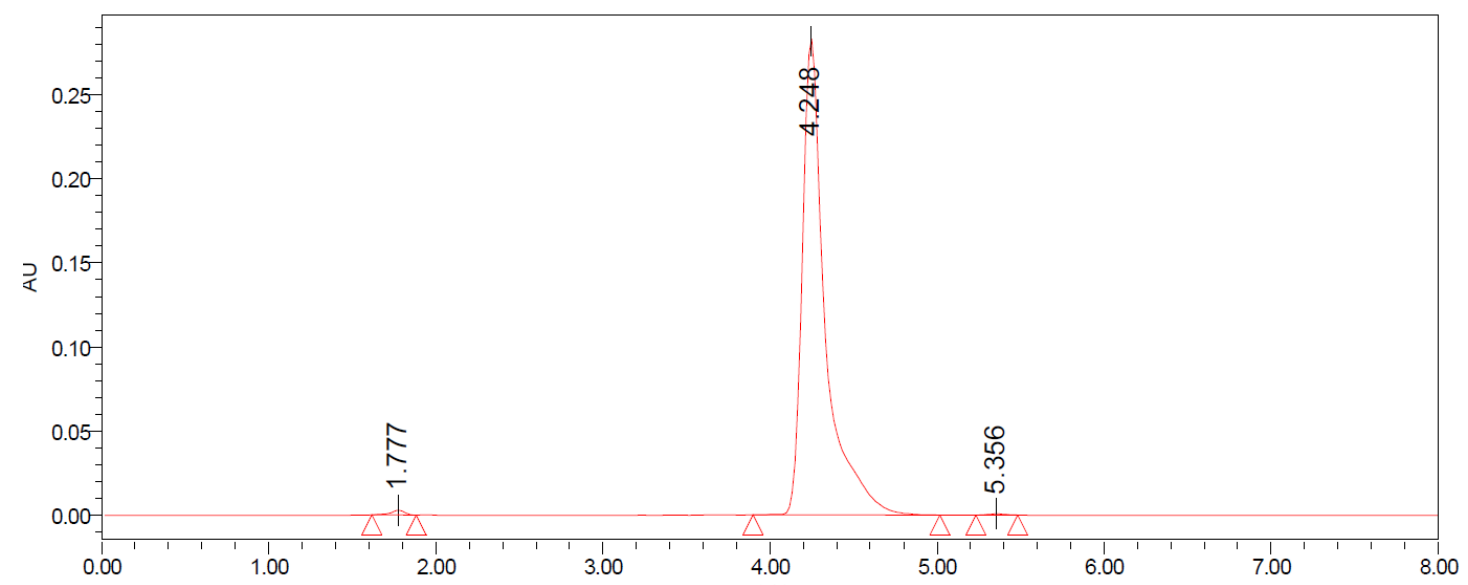

Figure S16. HPLC spectrum of compound 20 


\begin{tabular}{|c|c|c|c|}
\hline Instrument: & Waters e2695 & Column: & $\begin{array}{c}\text { Phenomenex column, C-18, } \\
5.0 \mu \mathrm{m}, 4.6 * 250 \mathrm{~mm}\end{array}$ \\
\hline Mobile Phase: & $\begin{array}{c}\text { Phase A: water; } \\
\text { Phase B: acetonitrile; } \\
\text { A/ B }=60 / 40, \mathrm{v} / \mathrm{v}\end{array}$ & Flow rate: & $1.0 \mathrm{~mL} / \mathrm{min}$ \\
\hline Ret. Time (min): & 4.038 & Purity $(\%)$ & 97.05 \\
\hline
\end{tabular}

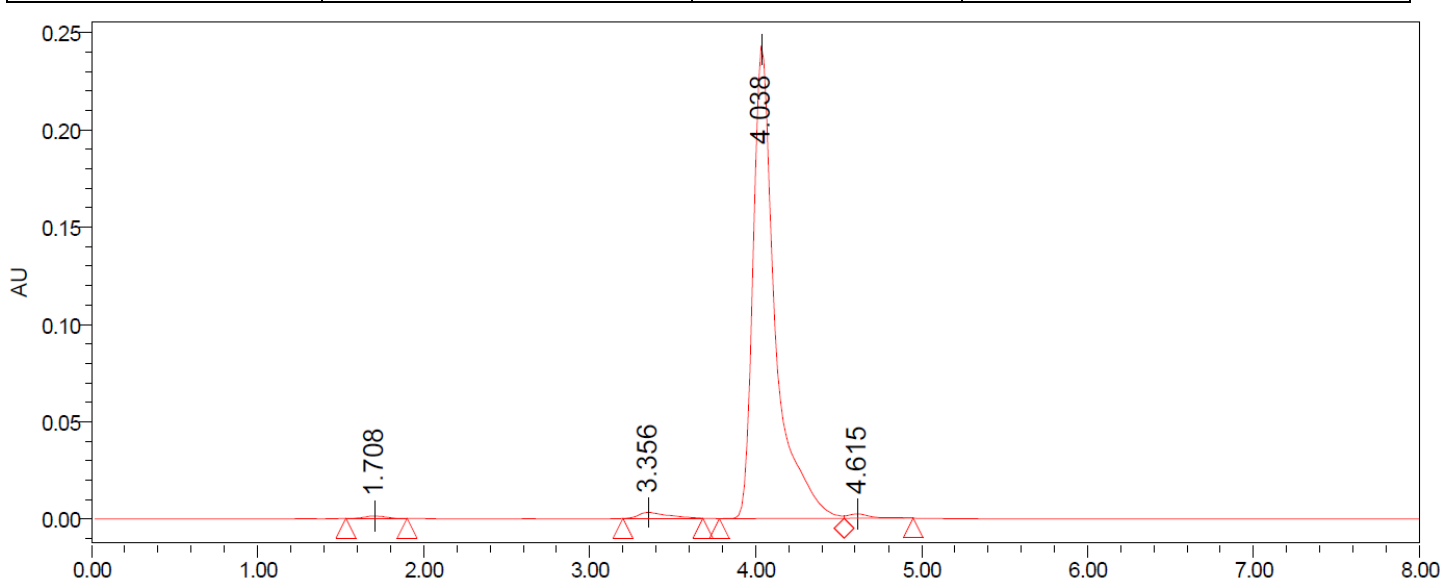

Figure S17. HPLC spectrum of compound 21

\begin{tabular}{|c|c|c|c|}
\hline Instrument: & Waters e2695 & Column: & $\begin{array}{c}\text { Phenomenex column, C-18, } \\
5.0 \mu \mathrm{m}, 4.6 * 250 \mathrm{~mm}\end{array}$ \\
\hline Mobile Phase: & $\begin{array}{c}\text { Phase A: water; } \\
\text { Phase B: acetonitrile; } \\
\text { A/ B = 60/40, v/v }\end{array}$ & Flow rate: & $1.0 \mathrm{~mL} / \mathrm{min}$ \\
\hline Ret. Time (min): & 4.958 & Purity (\%) & 99.82 \\
\hline
\end{tabular}

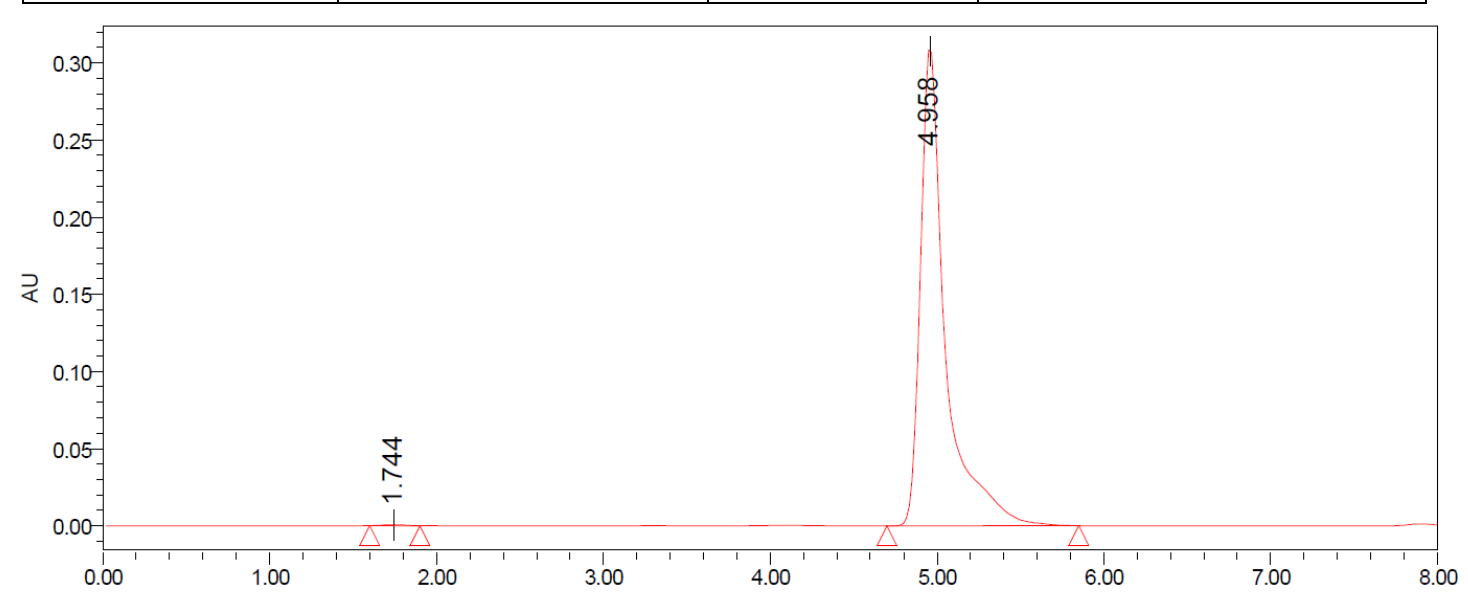

Figure S18. HPLC spectrum of compound 22 


\begin{tabular}{|c|c|c|c|}
\hline Instrument: & Waters e2695 & Column: & $\begin{array}{c}\text { Phenomenex column, C-18, } \\
5.0 \mu \mathrm{m}, 4.6 * 250 \mathrm{~mm}\end{array}$ \\
\hline Mobile Phase: & $\begin{array}{c}\text { Phase A: water; } \\
\text { Phase B: acetonitrile; } \\
\text { A/ B = 60/40, v/v }\end{array}$ & Flow rate: & $1.0 \mathrm{~mL} / \mathrm{min}$ \\
\hline Ret. Time (min): & 4.873 & Purity (\%) & 99.57 \\
\hline
\end{tabular}

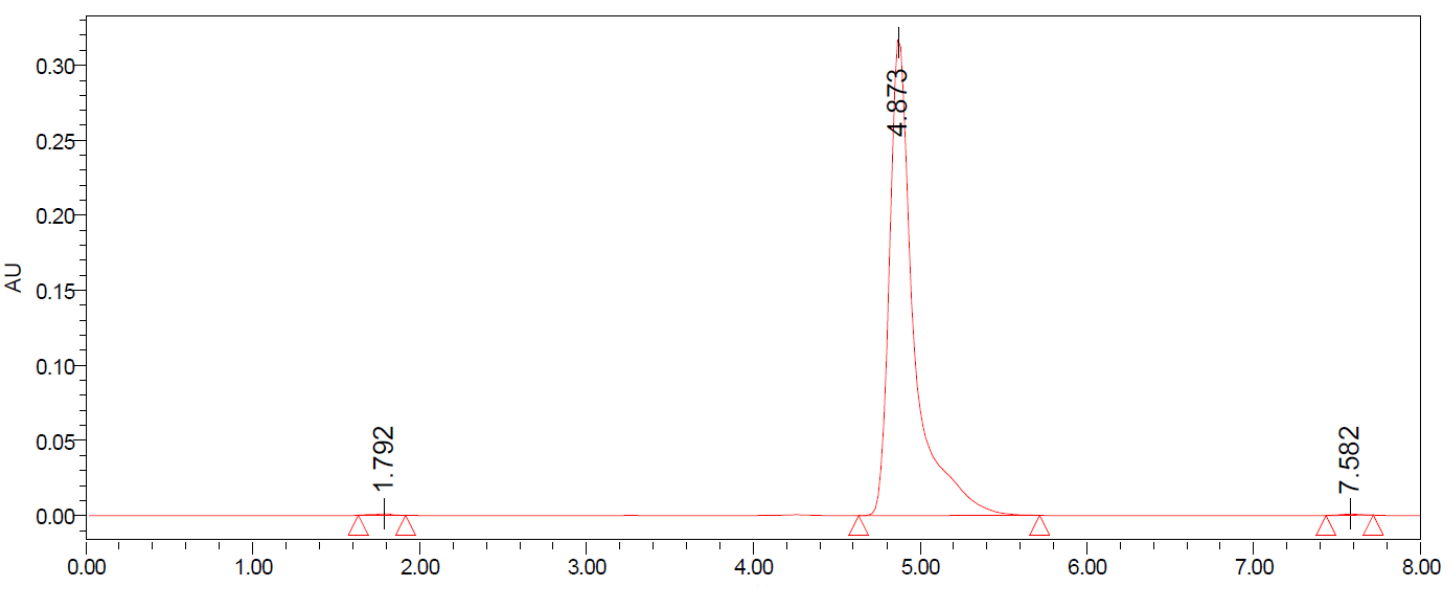

Figure S19. HPLC spectrum of compound 23

\begin{tabular}{|c|c|c|c|}
\hline Instrument: & Waters e2695 & Column: & $\begin{array}{c}\text { Phenomenex column, C-18, } \\
5.0 \mu \mathrm{m}, 4.6 * 250 \mathrm{~mm}\end{array}$ \\
\hline Mobile Phase: & $\begin{array}{c}\text { Phase A: water; } \\
\text { Phase B: acetonitrile; } \\
\mathrm{A} / \mathrm{B}=60 / 40, \mathrm{v} / \mathrm{v}\end{array}$ & Flow rate: & $1.0 \mathrm{~mL} / \mathrm{min}$ \\
\hline Ret. Time (min): & 4.047 & Purity (\%) & 99.17 \\
\hline
\end{tabular}

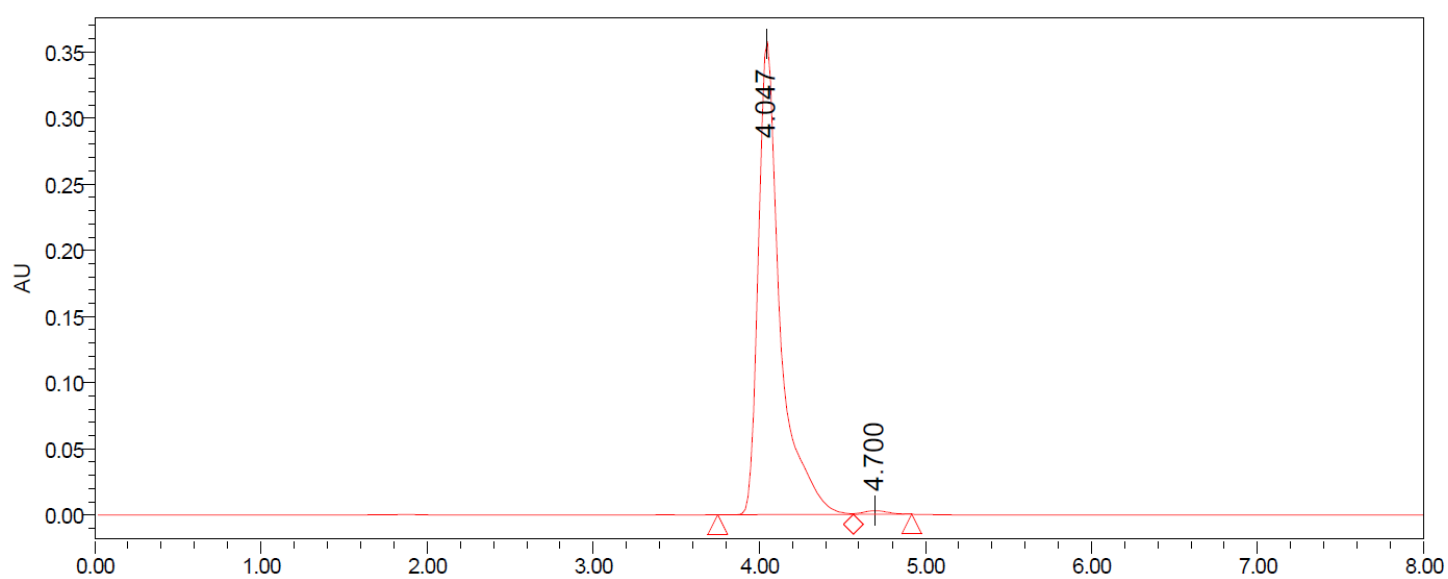

Figure S20. HPLC spectrum of compound 24 


\begin{tabular}{|c|c|c|c|}
\hline Instrument: & Waters e2695 & Column: & $\begin{array}{c}\text { Phenomenex column, C-18, } \\
5.0 \mu \mathrm{m}, 4.6 * 250 \mathrm{~mm}\end{array}$ \\
\hline Mobile Phase: & $\begin{array}{c}\text { Phase A: water; } \\
\text { Phase B: acetonitrile; } \\
\mathrm{A} / \mathrm{B}=60 / 40, \mathrm{v} / \mathrm{v}\end{array}$ & Flow rate: & $1.0 \mathrm{~mL} / \mathrm{min}$ \\
\hline Ret. Time (min): & 4.863 & Purity $(\%)$ & 99.86 \\
\hline
\end{tabular}

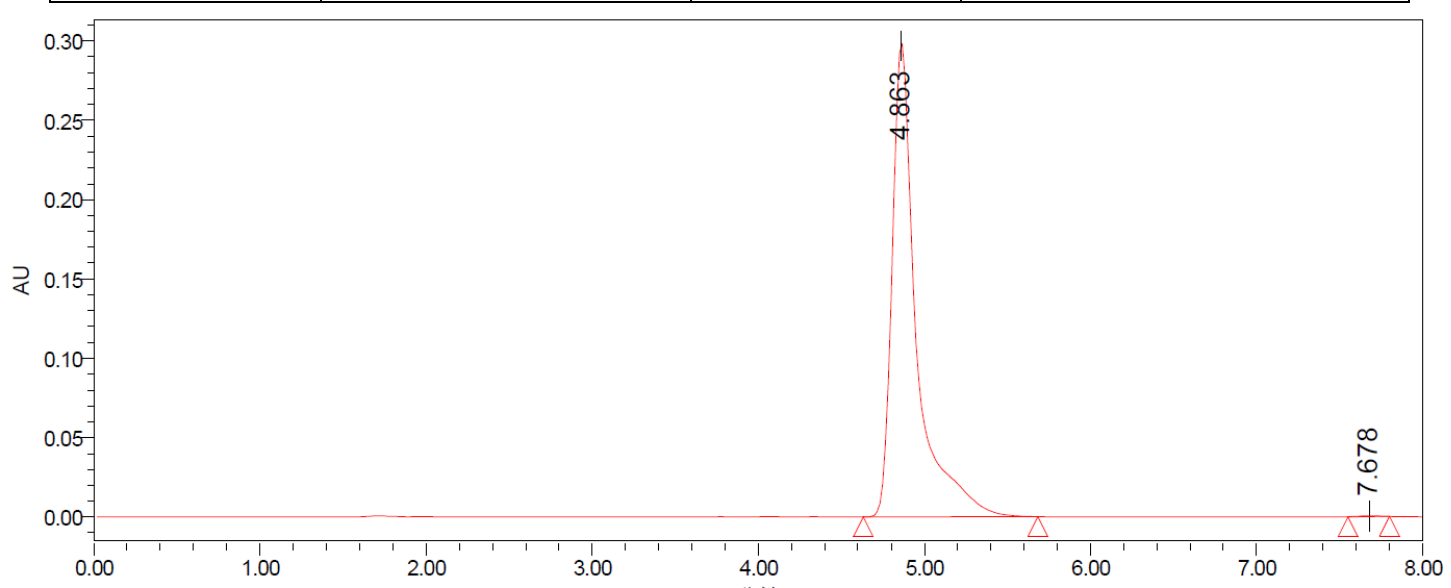

Figure S21. HPLC spectrum of compound 25

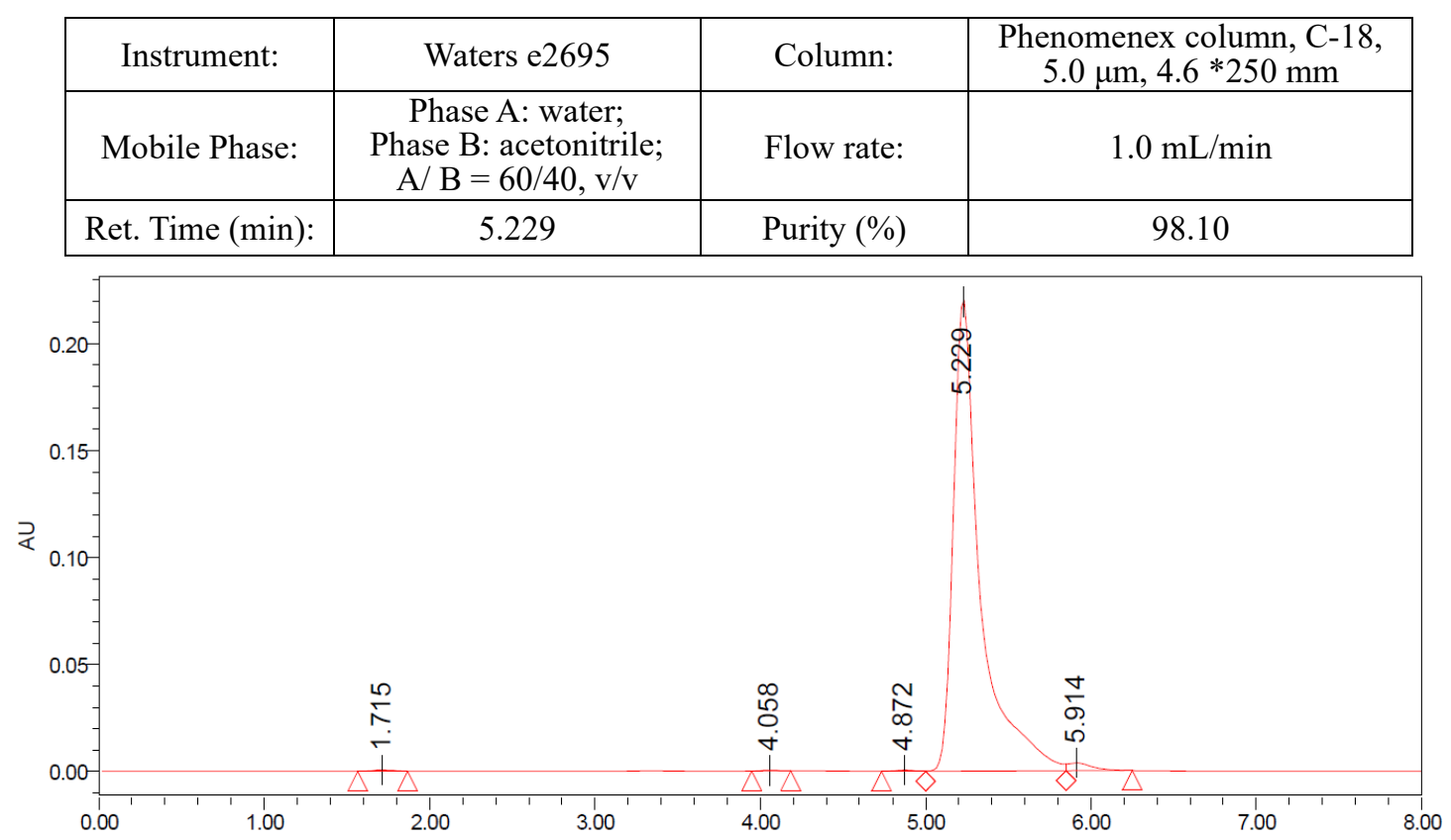

Figure S22. HPLC spectrum of compound $\mathbf{2 6}$ 


\begin{tabular}{|c|c|c|c|}
\hline Instrument: & Waters e2695 & Column: & $\begin{array}{c}\text { Phenomenex column, C-18, } \\
5.0 \mu \mathrm{m}, 4.6 * 250 \mathrm{~mm}\end{array}$ \\
\hline Mobile Phase: & $\begin{array}{c}\text { Phase A: water; } \\
\text { Phe B: acetonitrile; } \\
\text { A B = 45/55, } / \mathrm{v}\end{array}$ & Flow rate: & $1.0 \mathrm{~mL} / \mathrm{min}$ \\
\hline Ret. Time (min): & 4.848 & Purity (\%) & 99.33 \\
\hline
\end{tabular}

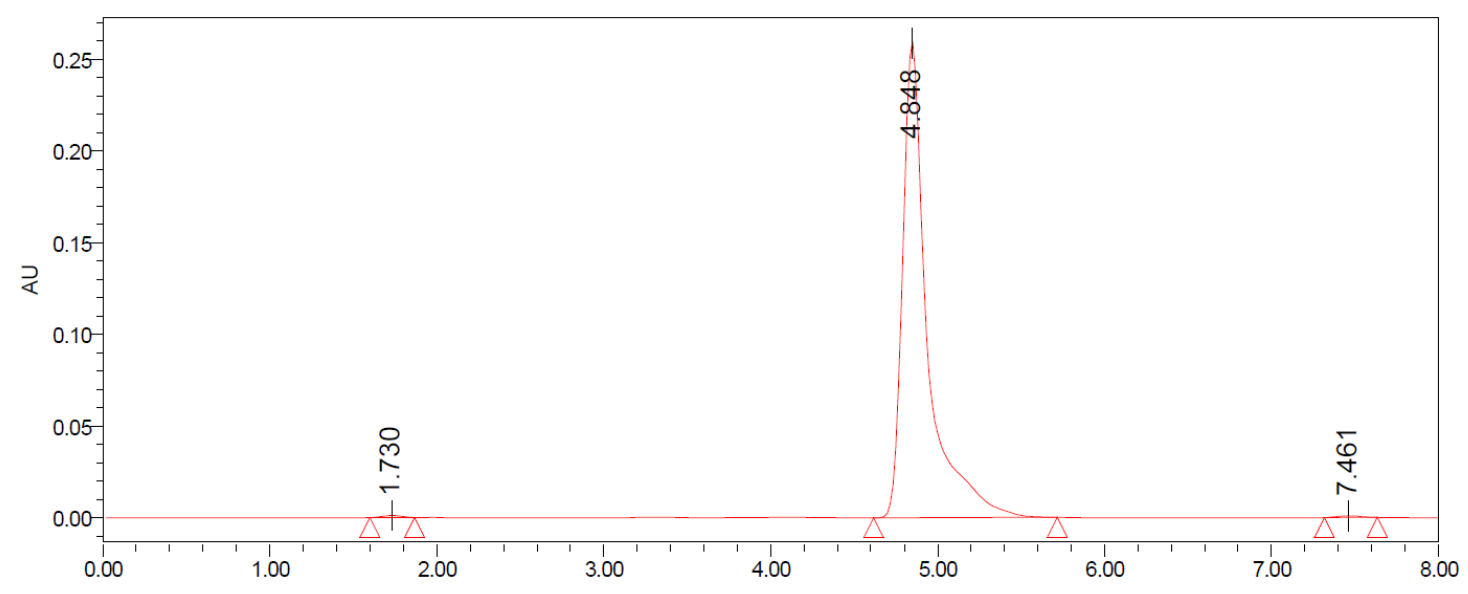

Figure S23. HPLC spectrum of compound 27

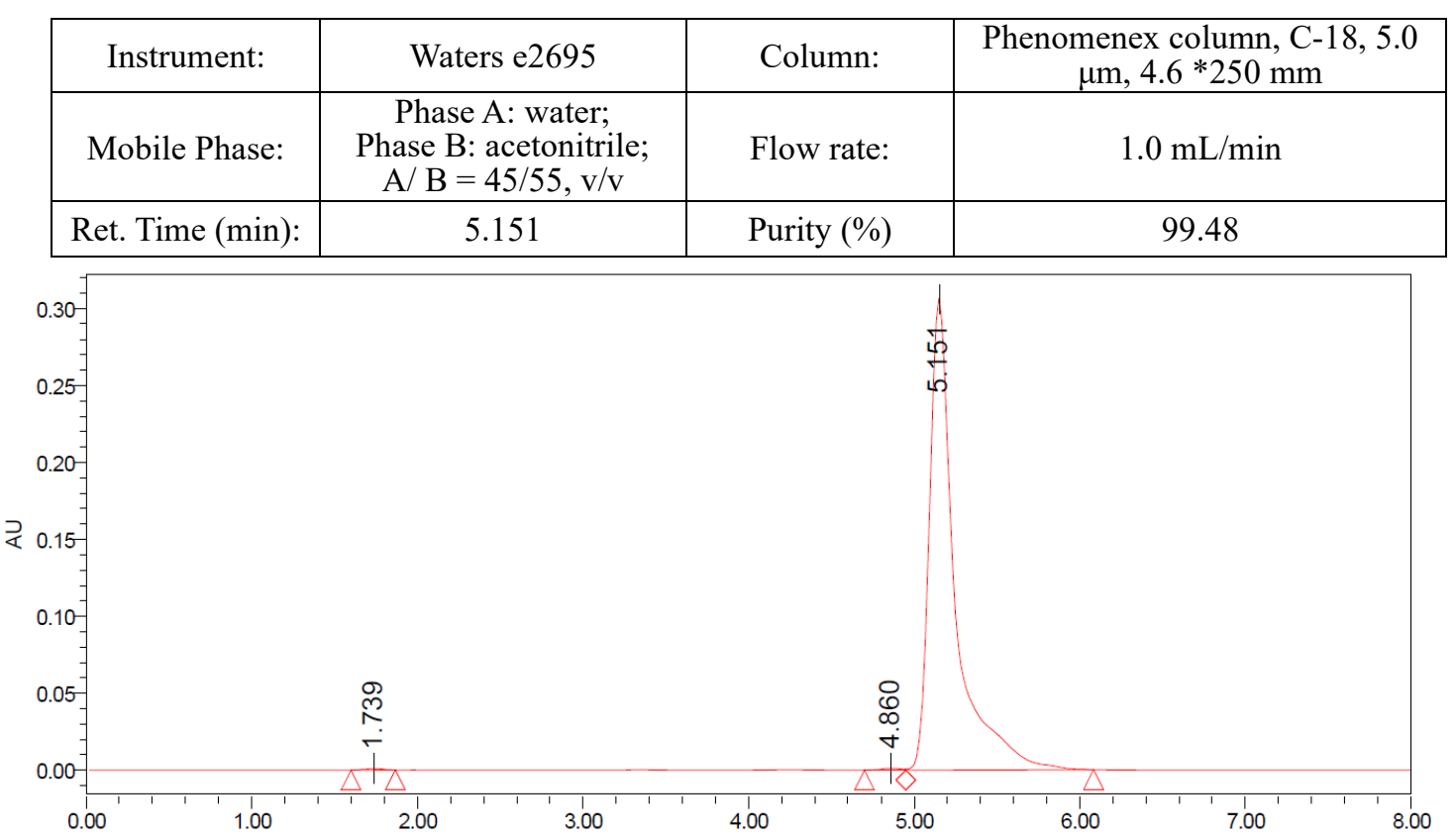

Figure S24. HPLC spectrum of compound $\mathbf{2 8}$ 


\begin{tabular}{|c|c|c|c|}
\hline Instrument: & Waters e2695 & Column: & $\begin{array}{c}\text { Phenomenex column, C-18, } \\
5.0 \mu \mathrm{m}, 4.6 * 250 \mathrm{~mm}\end{array}$ \\
\hline Mobile Phase: & $\begin{array}{c}\text { Phase A: water; } \\
\text { Phase B: acetonitrile; } \\
\text { A/ B }=45 / 55, \mathrm{v} / \mathrm{v}\end{array}$ & Flow rate: & $1.0 \mathrm{~mL} / \mathrm{min}$ \\
\hline Ret. Time (min): & 5.323 & Purity $(\%)$ & 98.18 \\
\hline
\end{tabular}

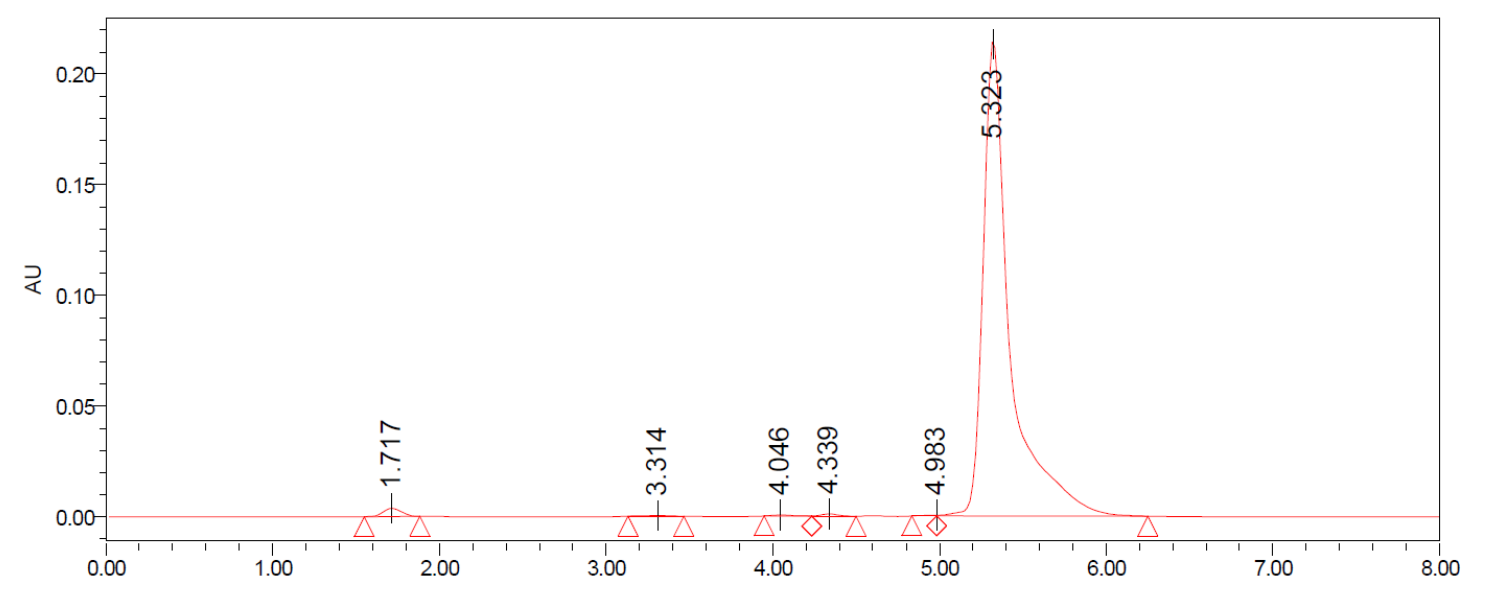

Figure S25. HPLC spectrum of compound 29

\begin{tabular}{|c|c|c|c|}
\hline Instrument: & Waters e2695 & Column: & $\begin{array}{c}\text { Phenomenex column, C-18, } \\
5.0 \mu \mathrm{m}, 4.6 * 250 \mathrm{~mm}\end{array}$ \\
\hline Mobile Phase: & $\begin{array}{c}\text { Phase A: water; } \\
\text { Phase B: acetonitrile; } \\
\text { A/ B = 45/55, v/v }\end{array}$ & Flow rate: & $1.0 \mathrm{~mL} / \mathrm{min}$ \\
\hline Ret. Time (min): & 4.645 & Purity (\%) & 96.76 \\
\hline
\end{tabular}

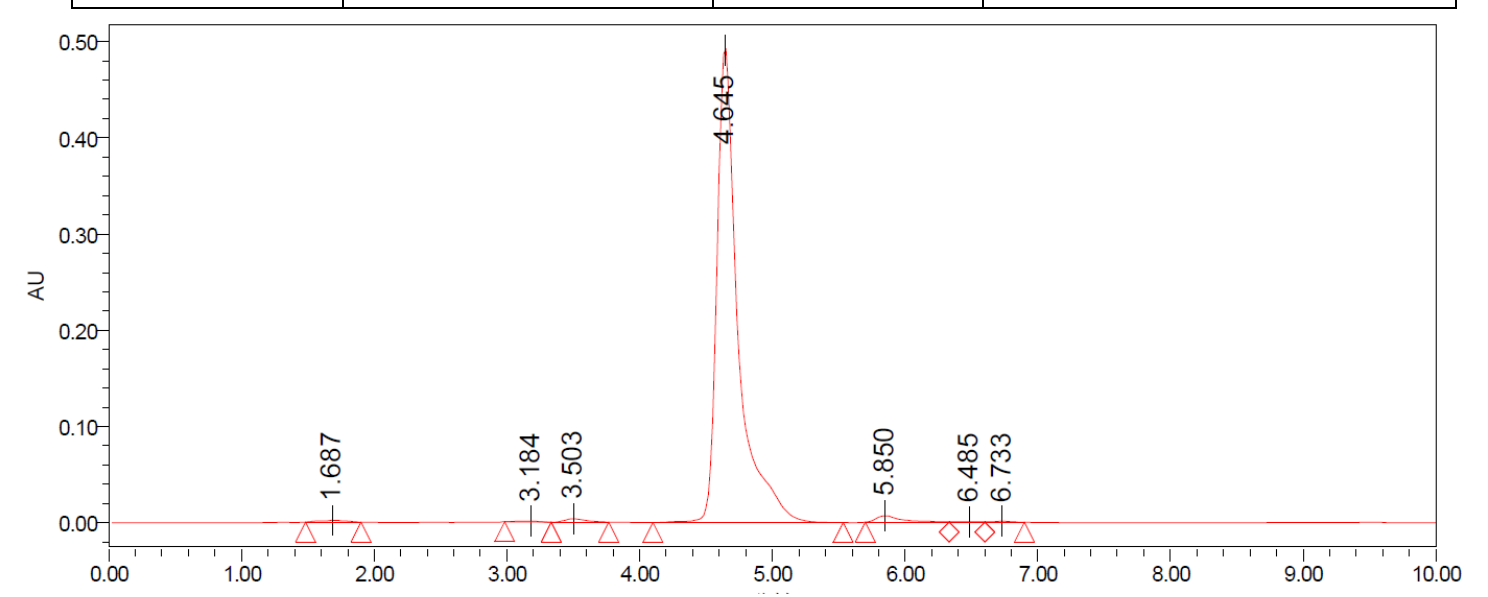

Figure S26. HPLC spectrum of compound $\mathbf{3 0}$ 


\begin{tabular}{|c|c|c|c|}
\hline Instrument: & Waters e2695 & Column: & $\begin{array}{c}\text { Phenomenex column, C-18, } \\
5.0 \mu \mathrm{m}, 4.6 * 250 \mathrm{~mm}\end{array}$ \\
\hline Mobile Phase: & $\begin{array}{c}\text { Phase A: water; } \\
\text { Phase B: acetonitrile; } \\
\text { A/ B }=45 / 55, \mathrm{v} / \mathrm{v}\end{array}$ & Flow rate: & $1.0 \mathrm{~mL} / \mathrm{min}$ \\
\hline Ret. Time (min): & 4.360 & Purity $(\%)$ & 99.92 \\
\hline
\end{tabular}

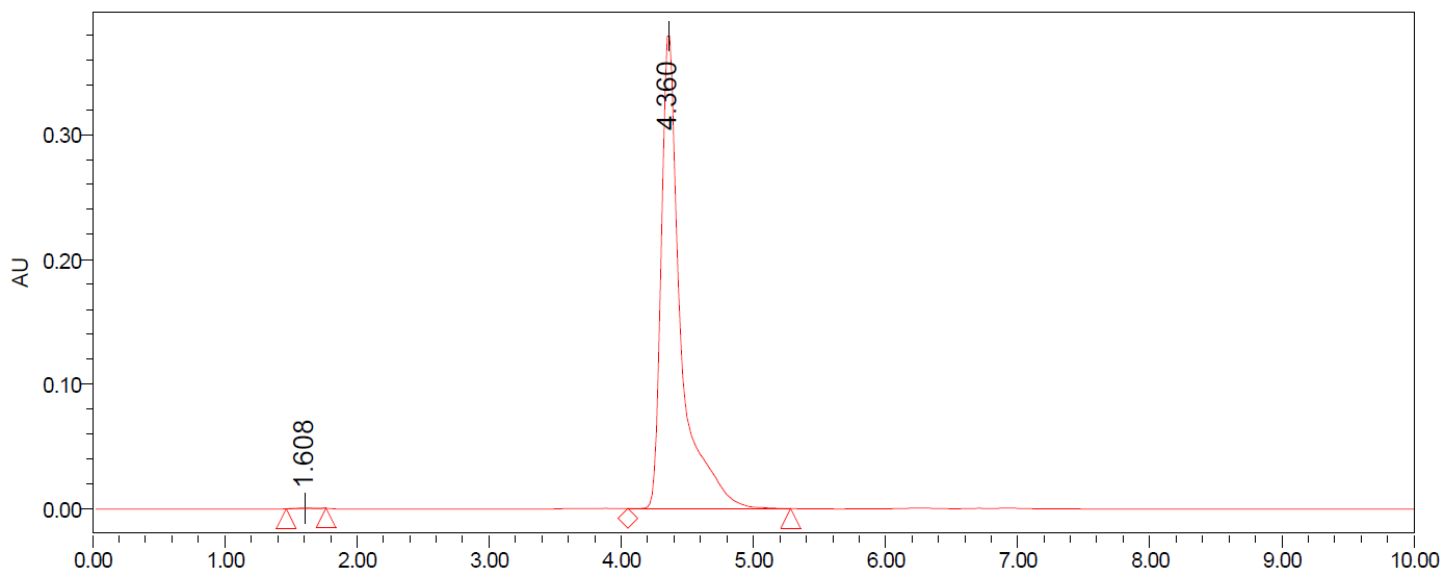

Figure S27. HPLC spectrum of compound 31

\begin{tabular}{|c|c|c|c|}
\hline Instrument: & Waters e2695 & Column: & $\begin{array}{c}\text { Phenomenex column, C-18, 5.0 } \\
\mu \mathrm{m}, 4.6 * 250 \mathrm{~mm}\end{array}$ \\
\hline Mobile Phase: & $\begin{array}{c}\text { Phase A: water; } \\
\text { Phase B: acetonitrile; } \\
\text { A/ B }=45 / 55, \mathrm{v} / \mathrm{v}\end{array}$ & Flow rate: & $1.0 \mathrm{~mL} / \mathrm{min}$ \\
\hline Ret. Time (min): & 3.790 & Purity (\%) & 98.74 \\
\hline
\end{tabular}

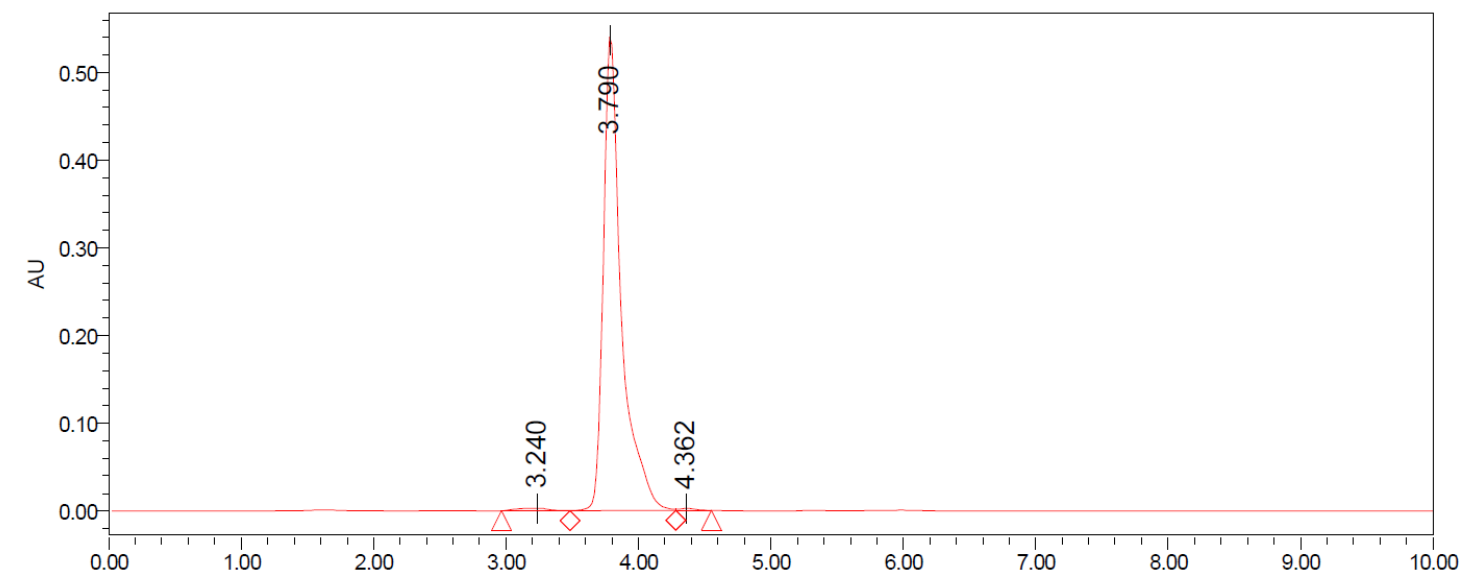

Figure S28. HPLC spectrum of compound 32 


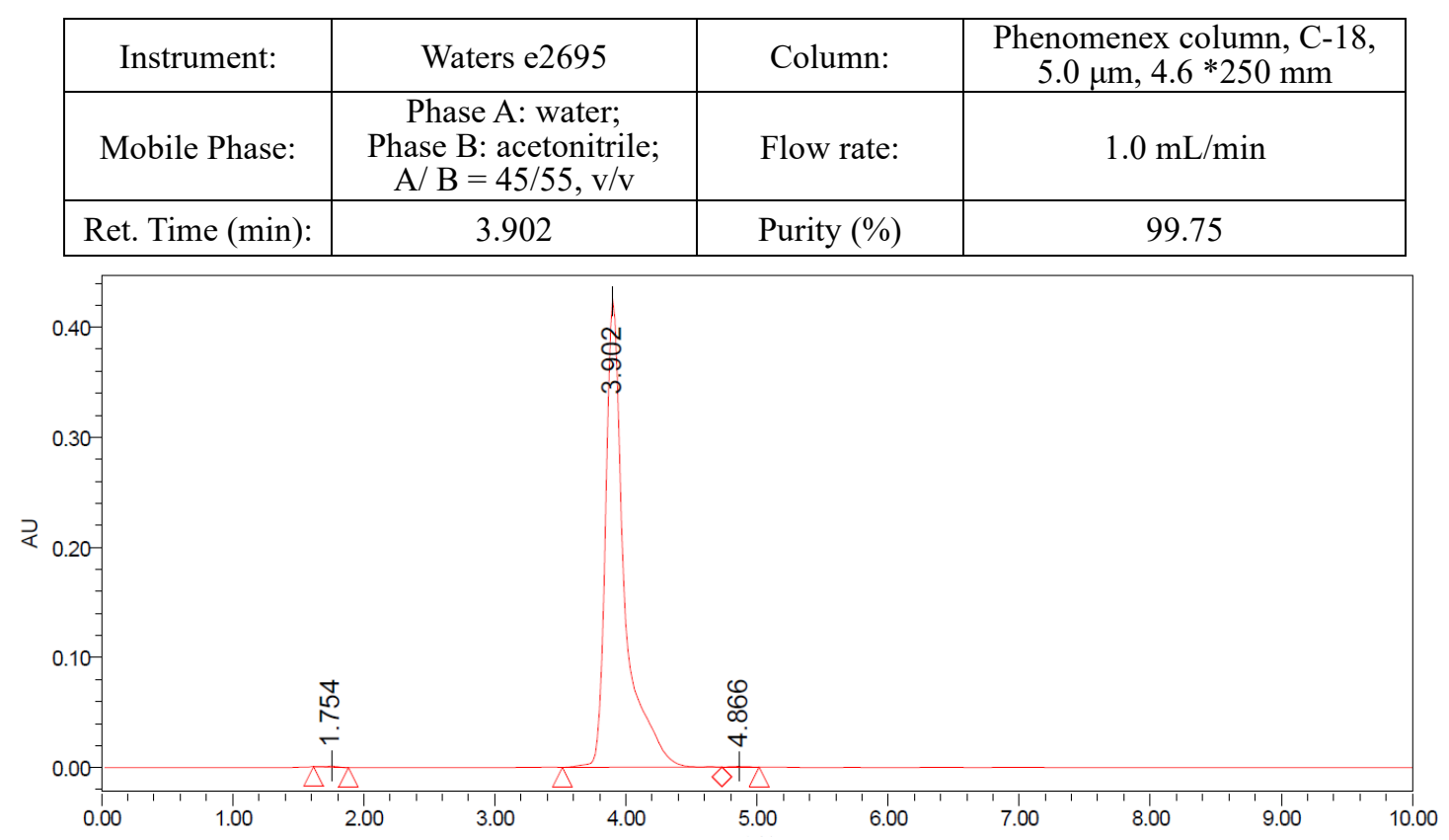

Figure S29. HPLC spectrum of compound $\mathbf{3 3}$

\begin{tabular}{|c|c|c|c|}
\hline Instrument: & Waters e2695 & Column: & $\begin{array}{c}\text { Phenomenex column, C-18, } \\
5.0 \mu \mathrm{m}, 4.6 * 250 \mathrm{~mm}\end{array}$ \\
\hline Mobile Phase: & $\begin{array}{c}\text { Phase A: water; } \\
\text { Phase B: acetonitrile; } \\
\text { A/ B = 45/55, v/v }\end{array}$ & Flow rate: & $1.0 \mathrm{~mL} / \mathrm{min}$ \\
\hline Ret. Time (min): & 4.714 & Purity $(\%)$ & 99.22 \\
\hline
\end{tabular}

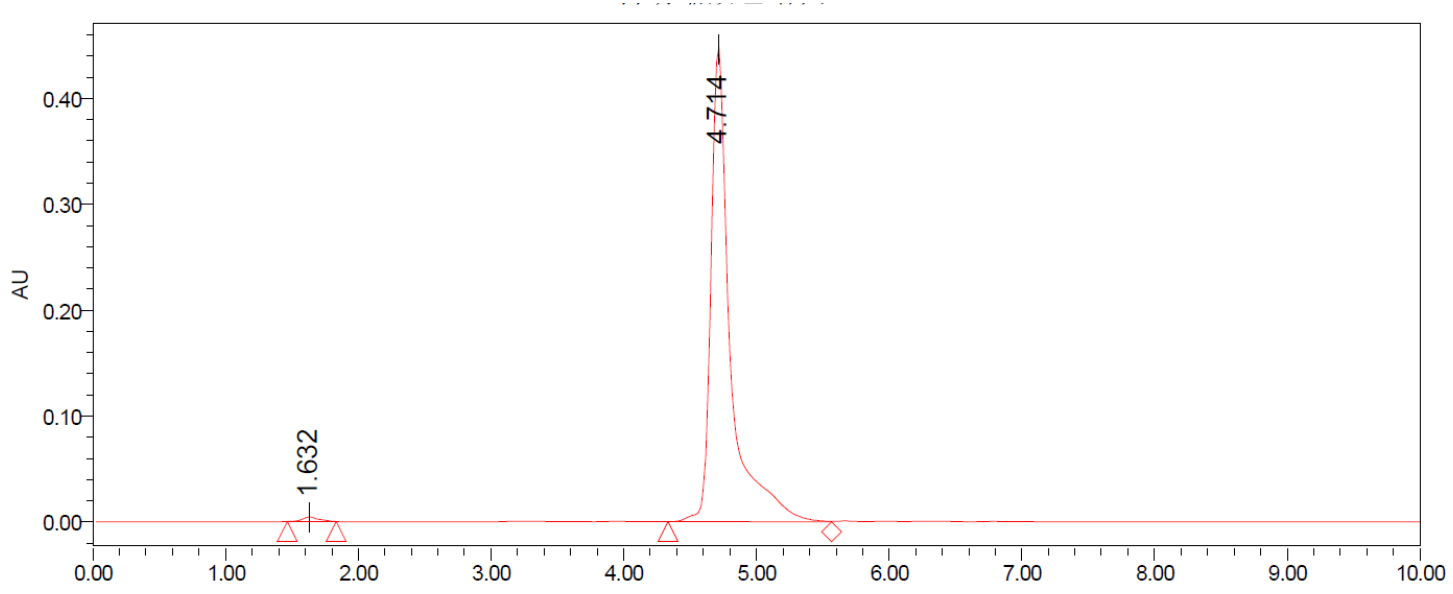

Figure S30. HPLC spectrum of compound 34 


\begin{tabular}{|c|c|c|c|}
\hline Instrument: & Waters e2695 & Column: & $\begin{array}{c}\text { Phenomenex column, C-18, } \\
5.0 \mu \mathrm{m}, 4.6 * 250 \mathrm{~mm}\end{array}$ \\
\hline Mobile Phase: & $\begin{array}{c}\text { Phase A: water } \\
\text { Phase B: acetonitrile; } \\
\text { A/ B }=45 / 55, \mathrm{v} / \mathrm{v}\end{array}$ & Flow rate: & $1.0 \mathrm{~mL} / \mathrm{min}$ \\
\hline Ret. Time (min): & 3.891 & Purity $(\%)$ & 99.17 \\
\hline
\end{tabular}

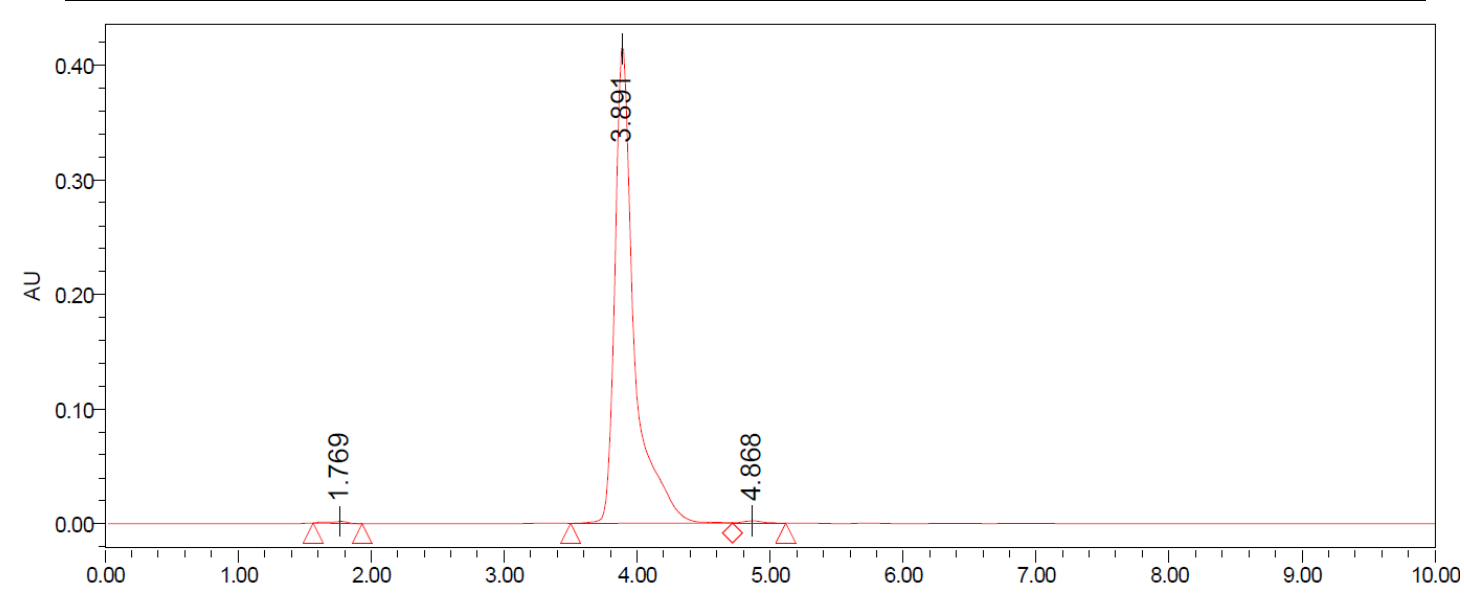

Figure S31. HPLC spectrum of compound 35

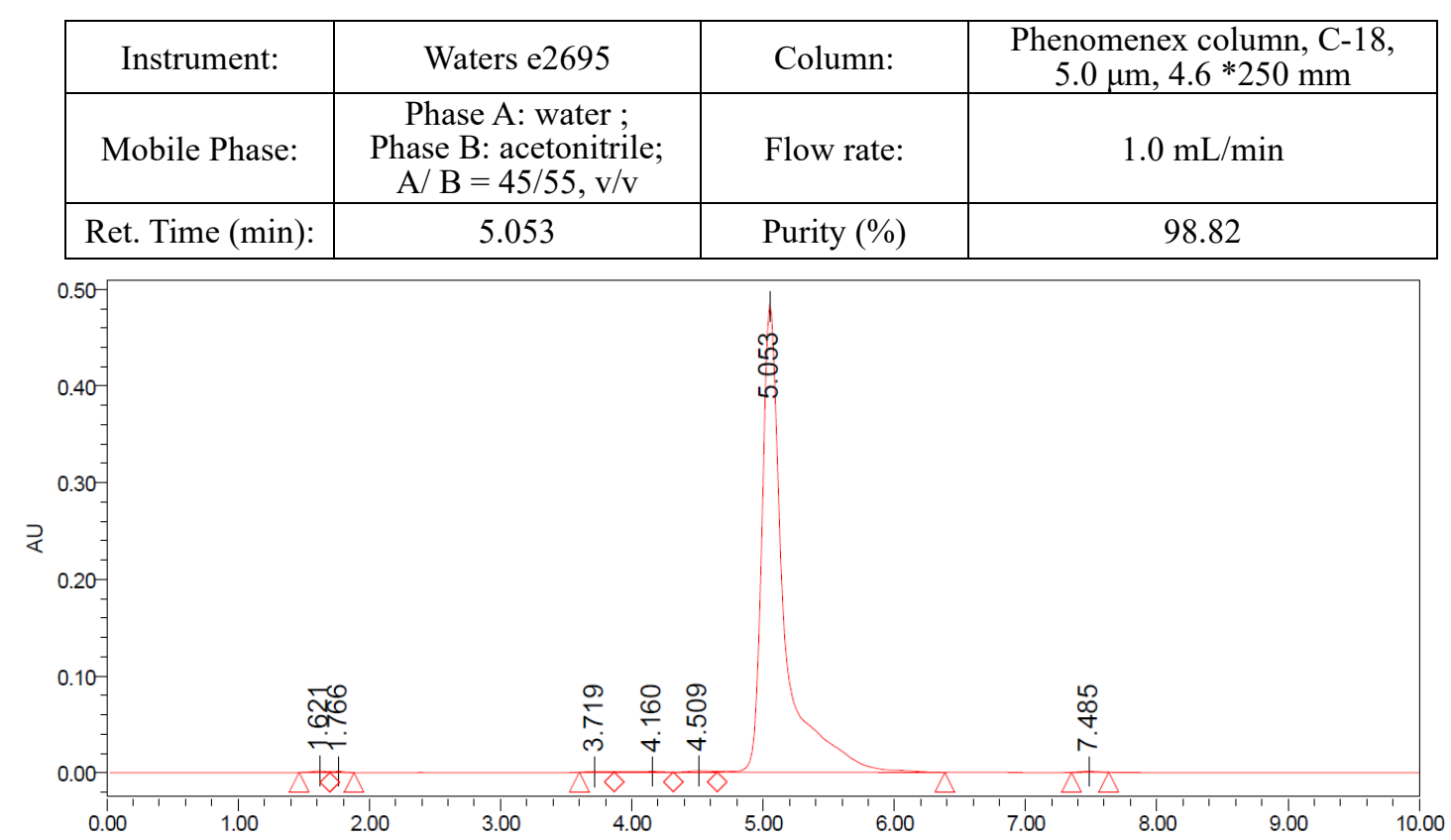

Figure S32. HPLC spectrum of compound $\mathbf{3 6}$ 


\begin{tabular}{|c|c|c|c|}
\hline Instrument: & Waters e2695 & Column: & $\begin{array}{c}\text { Phenomenex column, C-18, } \\
5.0 \mu \mathrm{m}, 4.6 * 250 \mathrm{~mm}\end{array}$ \\
\hline Mobile Phase: & $\begin{array}{c}\text { Phase A: water } \\
\text { Phase B: acetonitrile; } \\
\text { A/ B = 45/55, v/v }\end{array}$ & Flow rate: & $1.0 \mathrm{~mL} / \mathrm{min}$ \\
\hline Ret. Time (min): & 4.952 & Purity $(\%)$ & 97.45 \\
\hline
\end{tabular}

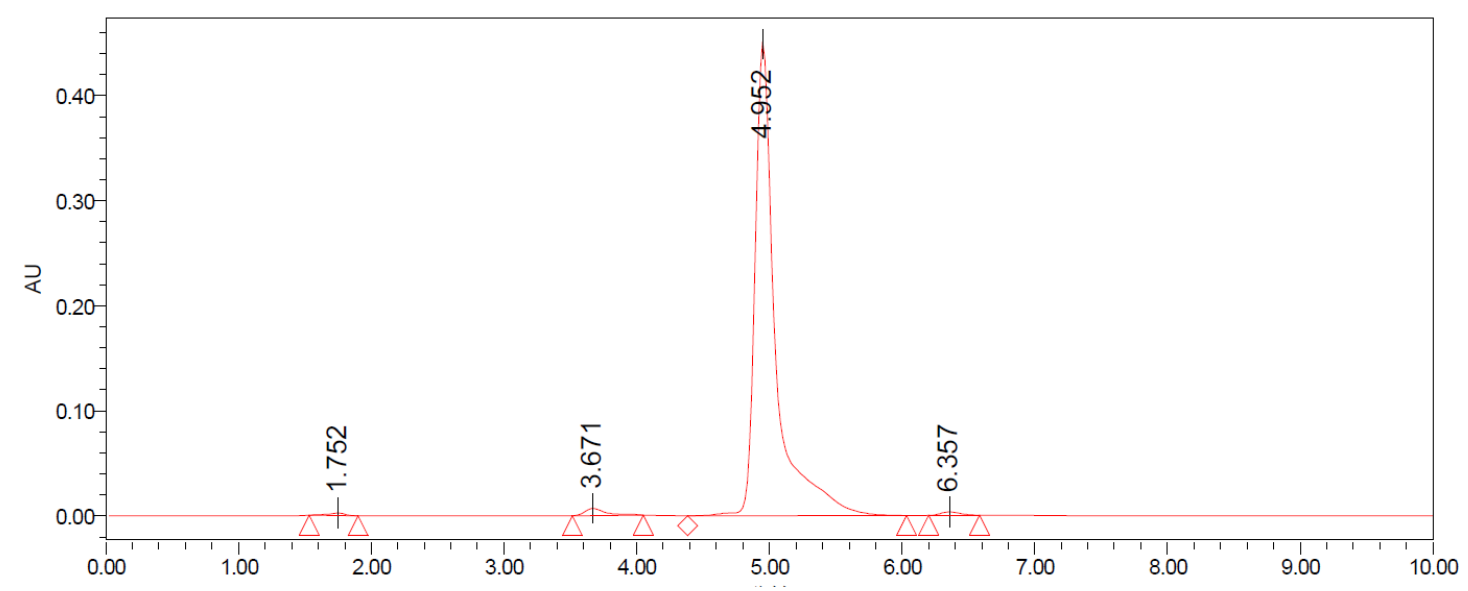

Figure S33. HPLC spectrum of compound $\mathbf{3 7}$

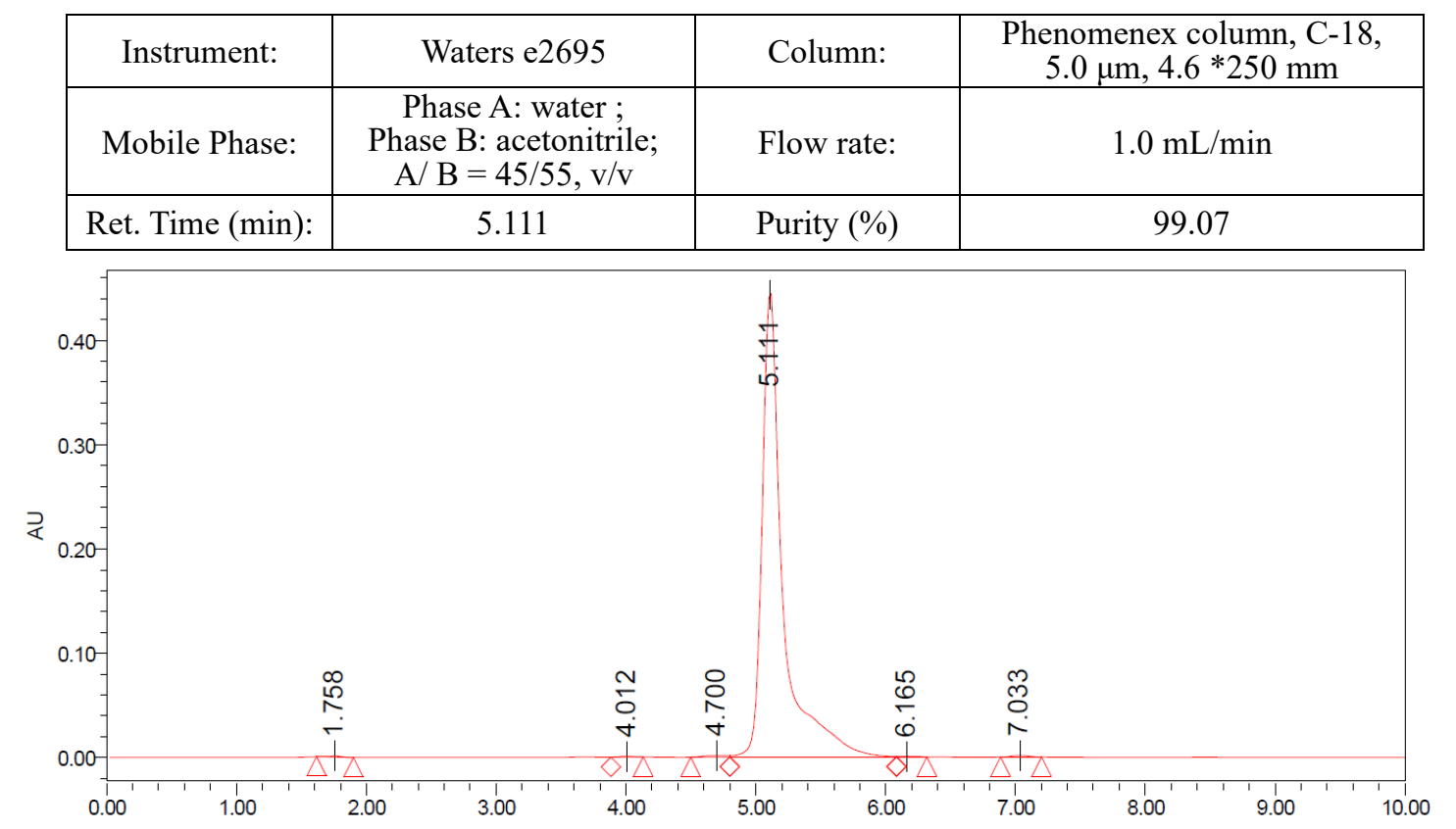

Figure S34. HPLC spectrum of compound $\mathbf{3 8}$ 


\begin{tabular}{|c|c|c|c|}
\hline Instrument: & Waters e2695 & Column: & $\begin{array}{c}\text { Phenomenex column, C-18, } \\
5.0 \mu \mathrm{m}, 4.6 * 250 \mathrm{~mm}\end{array}$ \\
\hline Mobile Phase: & $\begin{array}{c}\text { Phase A: water } \\
\text { Phase B: acetonitrile; } \\
\text { A/ B }=45 / 55, \mathrm{v} / \mathrm{v}\end{array}$ & Flow rate: & $1.0 \mathrm{~mL} / \mathrm{min}$ \\
\hline Ret. Time (min): & 4.597 & Purity $(\%)$ & 99.37 \\
\hline
\end{tabular}

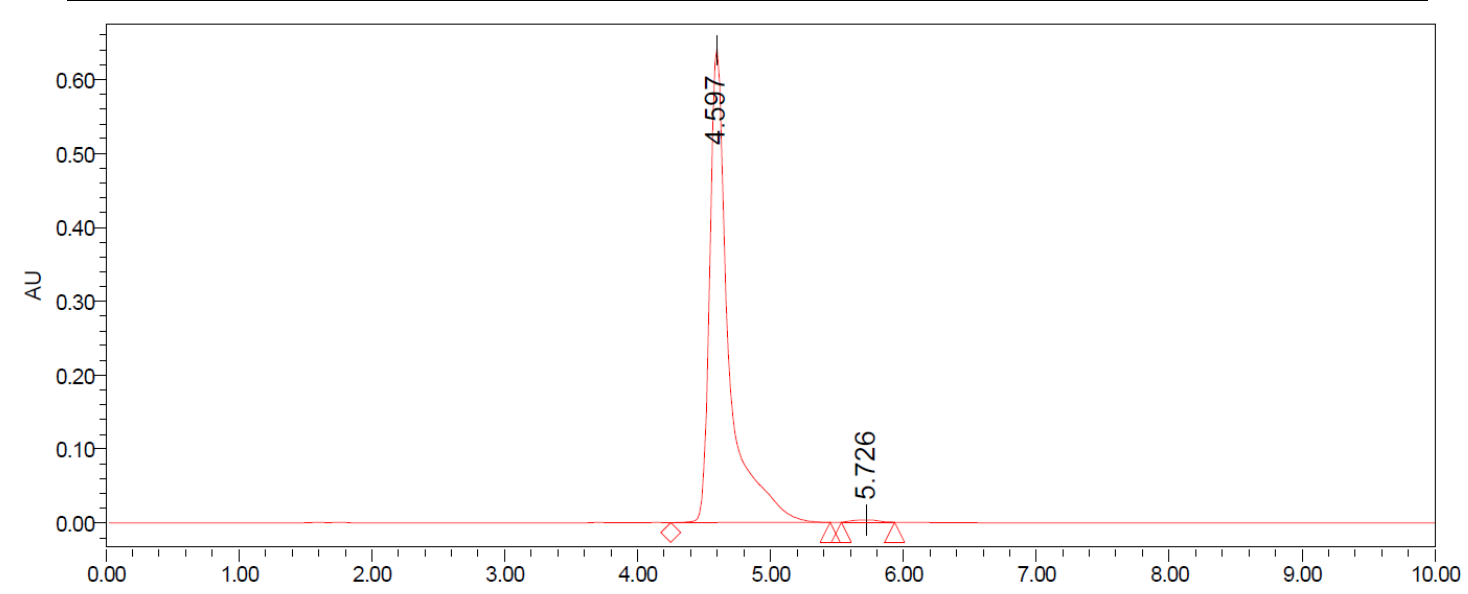

Figure S35. HPLC spectrum of compound 39

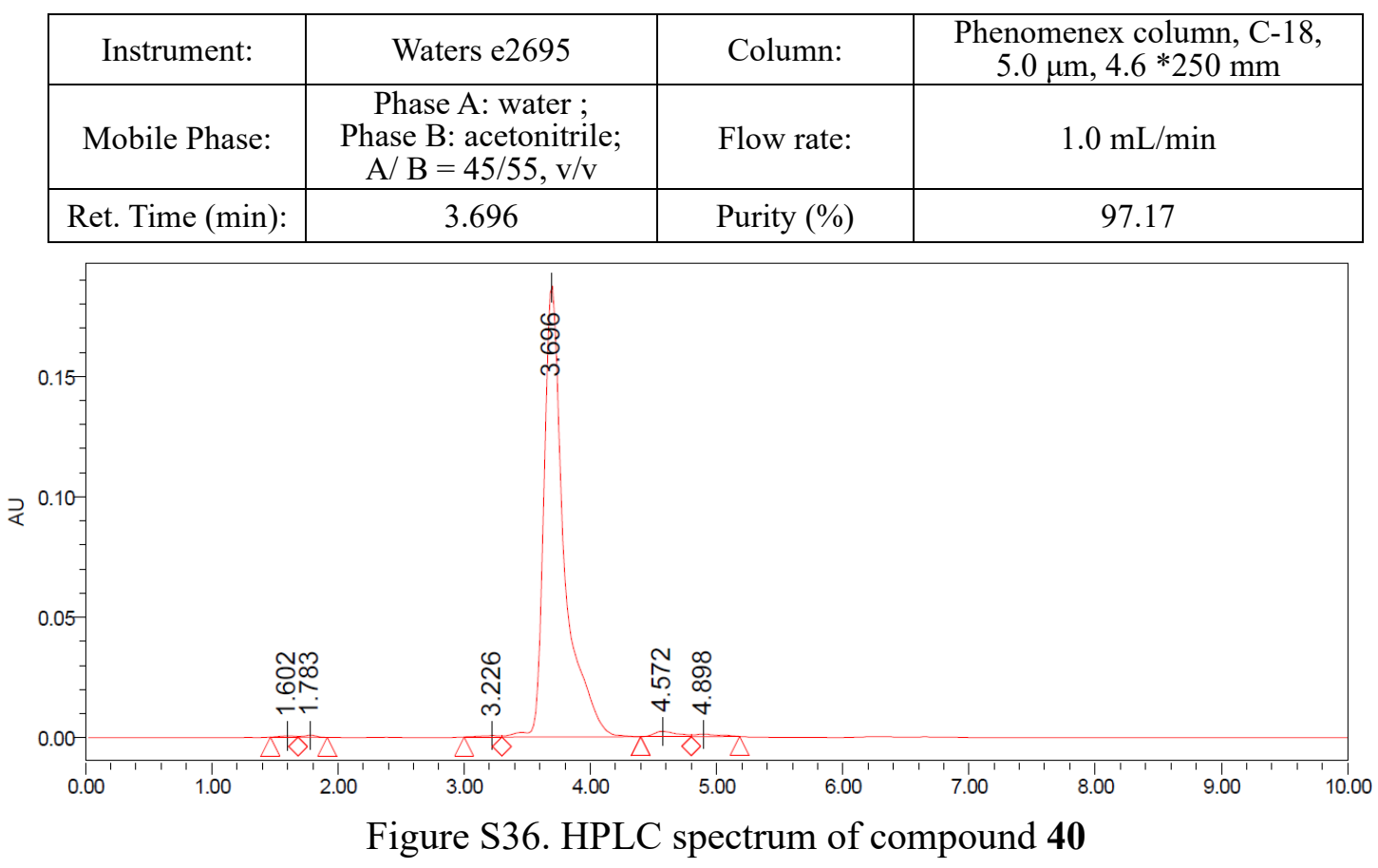




\begin{tabular}{|c|c|c|c|}
\hline Instrument: & Waters e2695 & Column: & $\begin{array}{c}\text { Phenomenex column, C-18, } \\
5.0 \mu \mathrm{m}, 4.6 * 250 \mathrm{~mm}\end{array}$ \\
\hline Mobile Phase: & $\begin{array}{c}\text { Phase A: water } \\
\text { Phase B: acetonitrile; } \\
\mathrm{A} / \mathrm{B}=45 / 55, \mathrm{v} / \mathrm{v}\end{array}$ & Flow rate: & $1.0 \mathrm{~mL} / \mathrm{min}$ \\
\hline Ret. Time (min): & 5.023 & Purity $(\%)$ & 100 \\
\hline
\end{tabular}

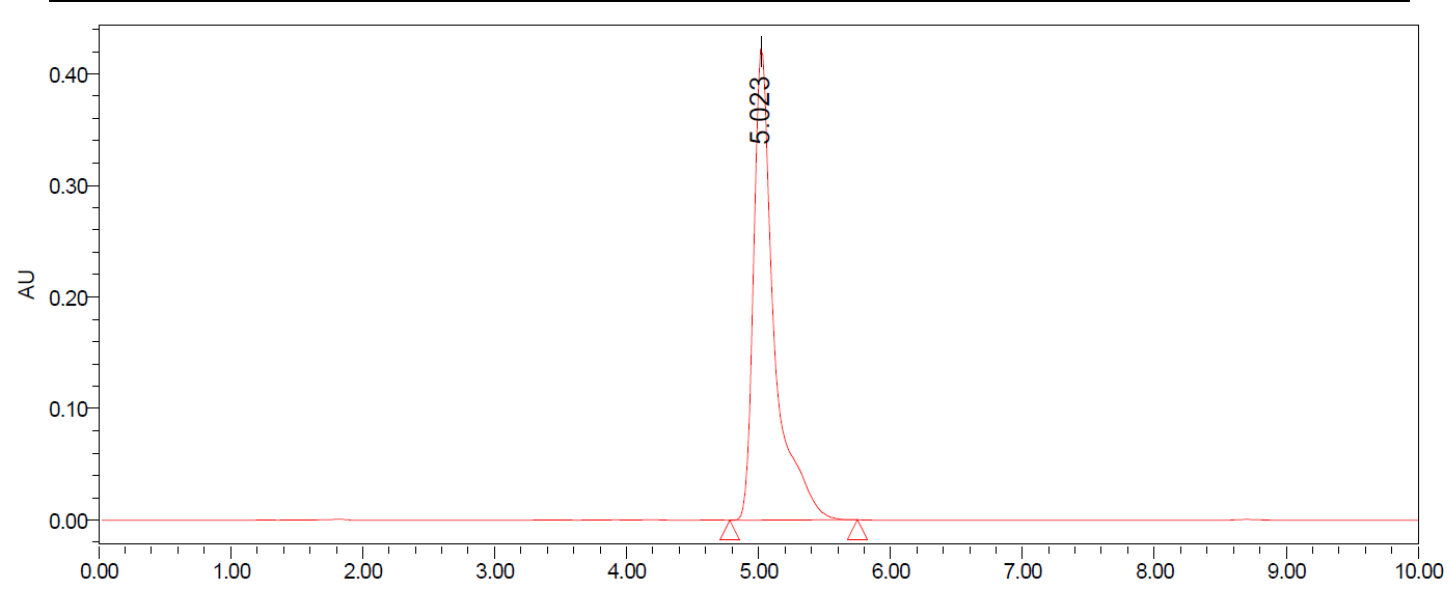

Figure S37. HPLC spectrum of compound 41

\begin{tabular}{|c|c|c|c|}
\hline Instrument: & Waters e2695 & Column: & $\begin{array}{c}\text { Phenomenex column, C-18, } \\
5.0 \mu \mathrm{m}, 4.6 * 250 \mathrm{~mm}\end{array}$ \\
\hline Mobile Phase: & $\begin{array}{c}\text { Phase A: water } \\
\text { Phase B: acetonitrile; } \\
\mathrm{A} / \mathrm{B}=30 / 70, \mathrm{v} / \mathrm{v}\end{array}$ & Flow rate: & $1.0 \mathrm{~mL} / \mathrm{min}$ \\
\hline Ret. Time (min): & 4.648 & Purity $(\%)$ & 98.45 \\
\hline
\end{tabular}

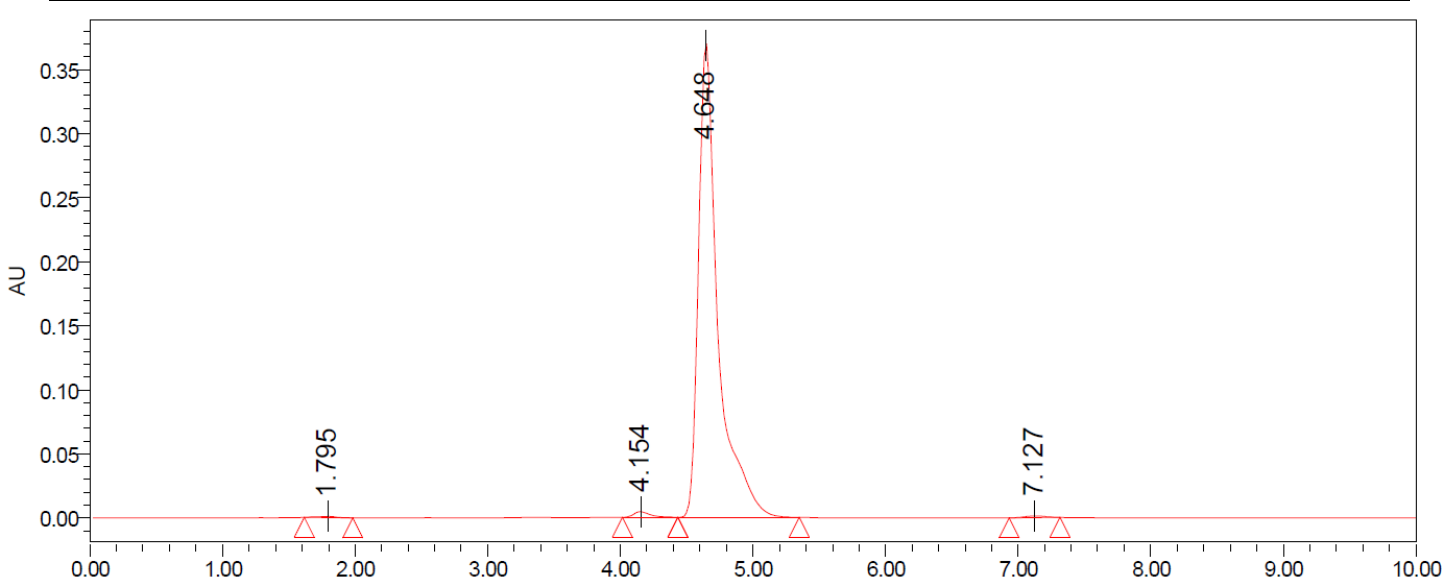

Figure S38. HPLC spectrum of compound $\mathbf{4 2}$ 


\begin{tabular}{|c|c|c|c|}
\hline Instrument: & Waters e2695 & Column: & $\begin{array}{c}\text { Phenomenex column, C-18, } \\
5.0 \mu \mathrm{m}, 4.6 * 250 \mathrm{~mm}\end{array}$ \\
\hline Mobile Phase: & $\begin{array}{c}\text { Phase A: water } \\
\text { Phase B: acetonitrile; } \\
\text { A/ B }=45 / 55, \mathrm{v} / \mathrm{v}\end{array}$ & Flow rate: & $1.0 \mathrm{~mL} / \mathrm{min}$ \\
\hline Ret. Time (min): & 3.405 & Purity (\%) & 98.96 \\
\hline
\end{tabular}

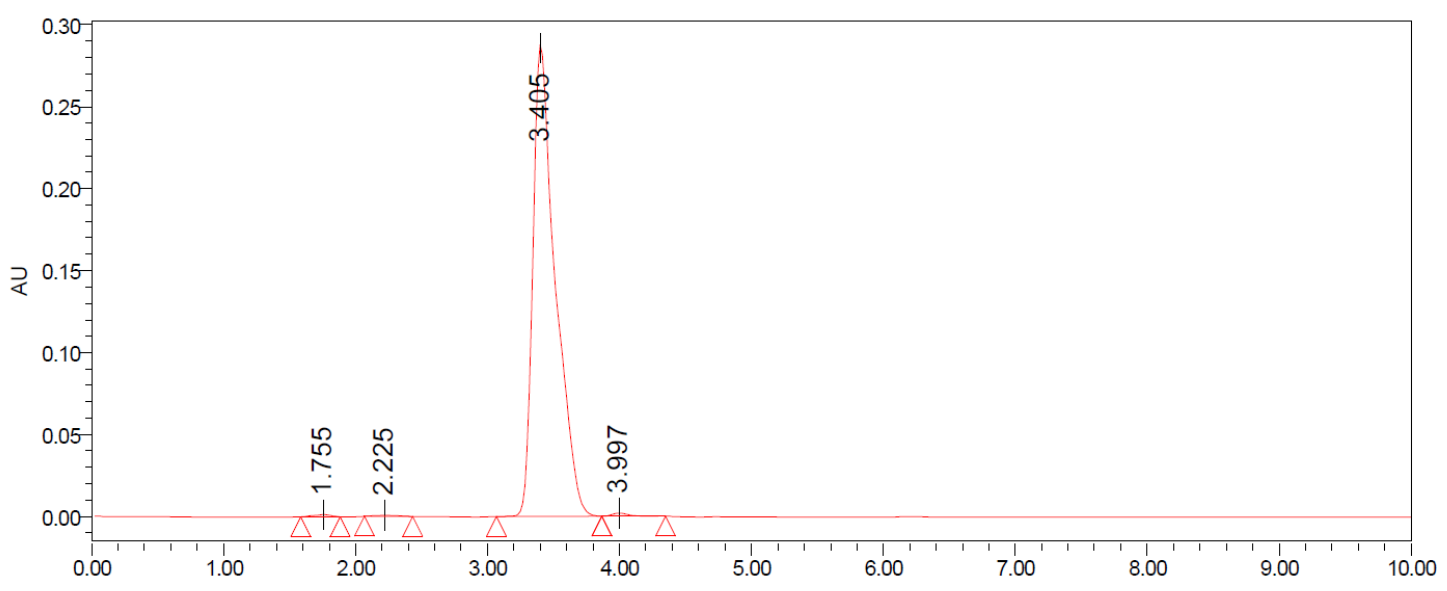

Figure S39. HPLC spectrum of compound $\mathbf{4 3}$

\begin{tabular}{|c|c|c|c|}
\hline Instrument: & Waters e2695 & Column: & $\begin{array}{c}\text { Phenomenex column, C-18, } \\
5.0 \mu \mathrm{m}, 4.6 * 250 \mathrm{~mm}\end{array}$ \\
\hline Mobile Phase: & $\begin{array}{c}\text { Phase A: water } ; \\
\text { Phase B: acetonitrile; } \\
\text { A/ B }=45 / 55, \mathrm{v} / \mathrm{v}\end{array}$ & Flow rate: & $1.0 \mathrm{~mL} / \mathrm{min}$ \\
\hline Ret. Time (min): & 4.194 & Purity (\%) & 99.44 \\
\hline
\end{tabular}

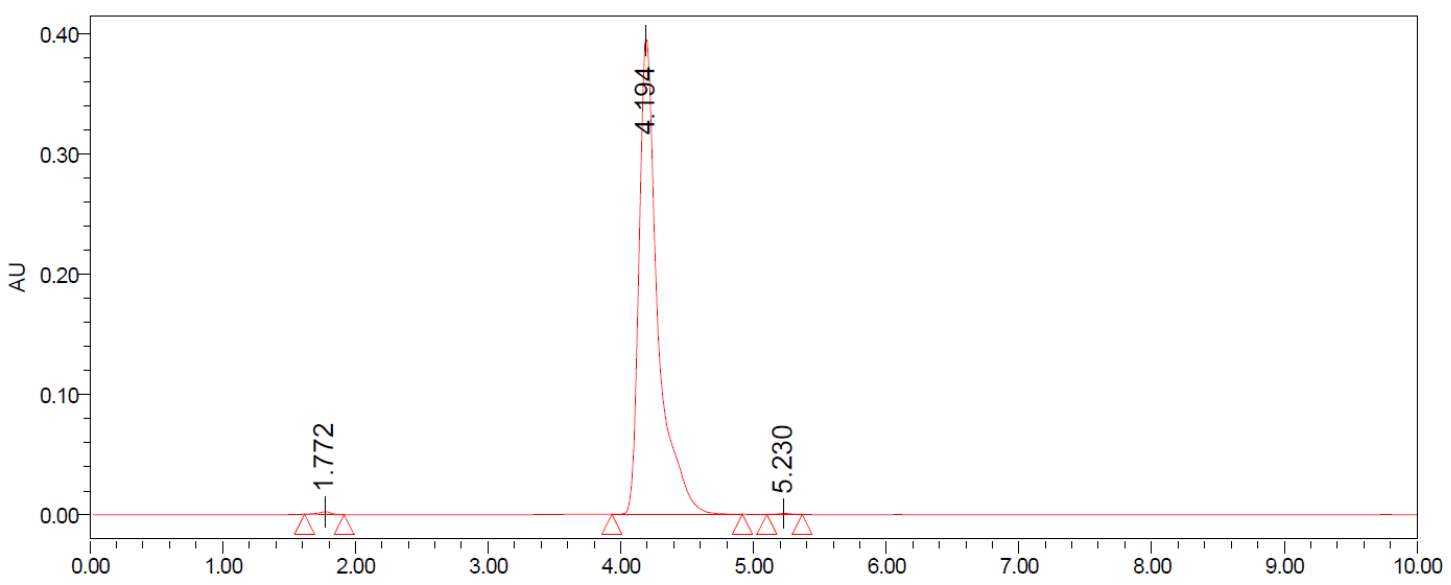

Figure S40. HPLC spectrum of compound 44 


\begin{tabular}{|c|c|c|c|}
\hline Instrument: & Waters e2695 & Column: & $\begin{array}{c}\text { Phenomenex column, C-18, } \\
5.0 \mu \mathrm{m}, 4.6 * 250 \mathrm{~mm}\end{array}$ \\
\hline Mobile Phase: & $\begin{array}{c}\text { Phase A: water } \\
\text { Phase B: acetonitrile; } \\
\text { A/ B }=45 / 55, \mathrm{v} / \mathrm{v}\end{array}$ & Flow rate: & $1.0 \mathrm{~mL} / \mathrm{min}$ \\
\hline Ret. Time (min): & 3.974 & Purity $(\%)$ & 96.90 \\
\hline
\end{tabular}

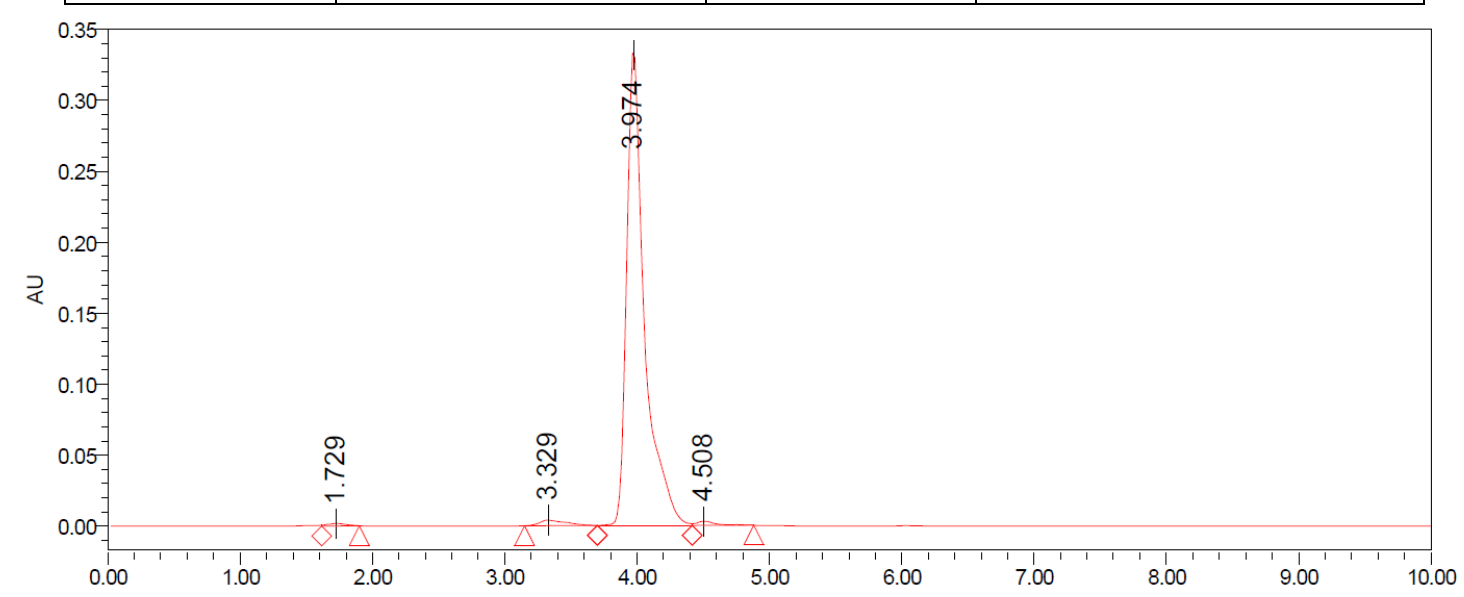

Figure S41. HPLC spectrum of compound $\mathbf{4 5}$

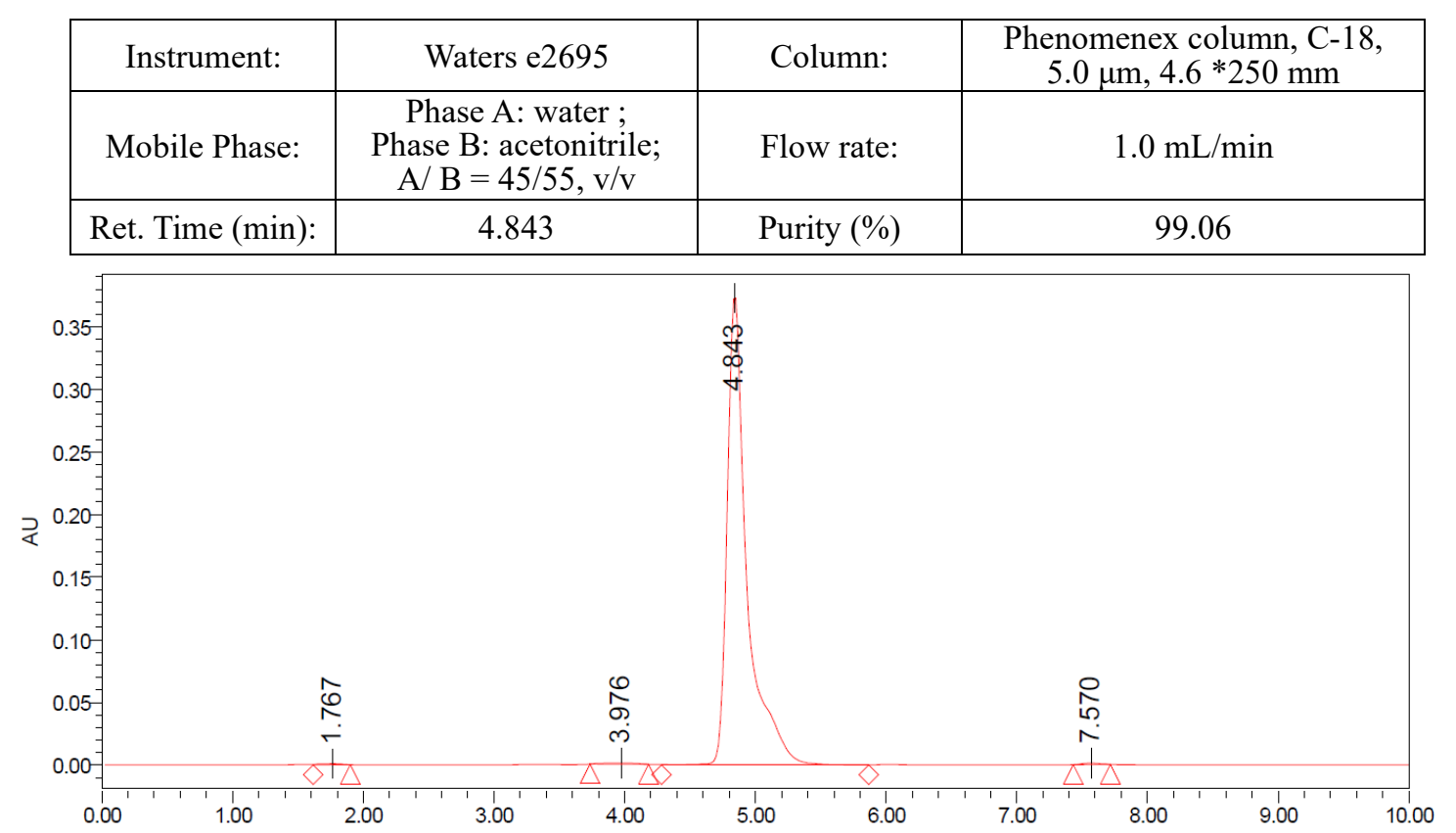

Figure S42. HPLC spectrum of compound 46 


\begin{tabular}{|c|c|c|c|}
\hline Instrument: & Waters e2695 & Column: & $\begin{array}{c}\text { Phenomenex column, C-18, } \\
5.0 \mu \mathrm{m}, 4.6 * 250 \mathrm{~mm}\end{array}$ \\
\hline Mobile Phase: & $\begin{array}{c}\text { Phase A: water } \\
\text { Phase B: acetonitrile; } \\
\mathrm{A} / \mathrm{B}=45 / 55, \mathrm{v} / \mathrm{v}\end{array}$ & Flow rate: & $1.0 \mathrm{~mL} / \mathrm{min}$ \\
\hline Ret. Time (min): & 4.812 & Purity $(\%)$ & 99.14 \\
\hline
\end{tabular}

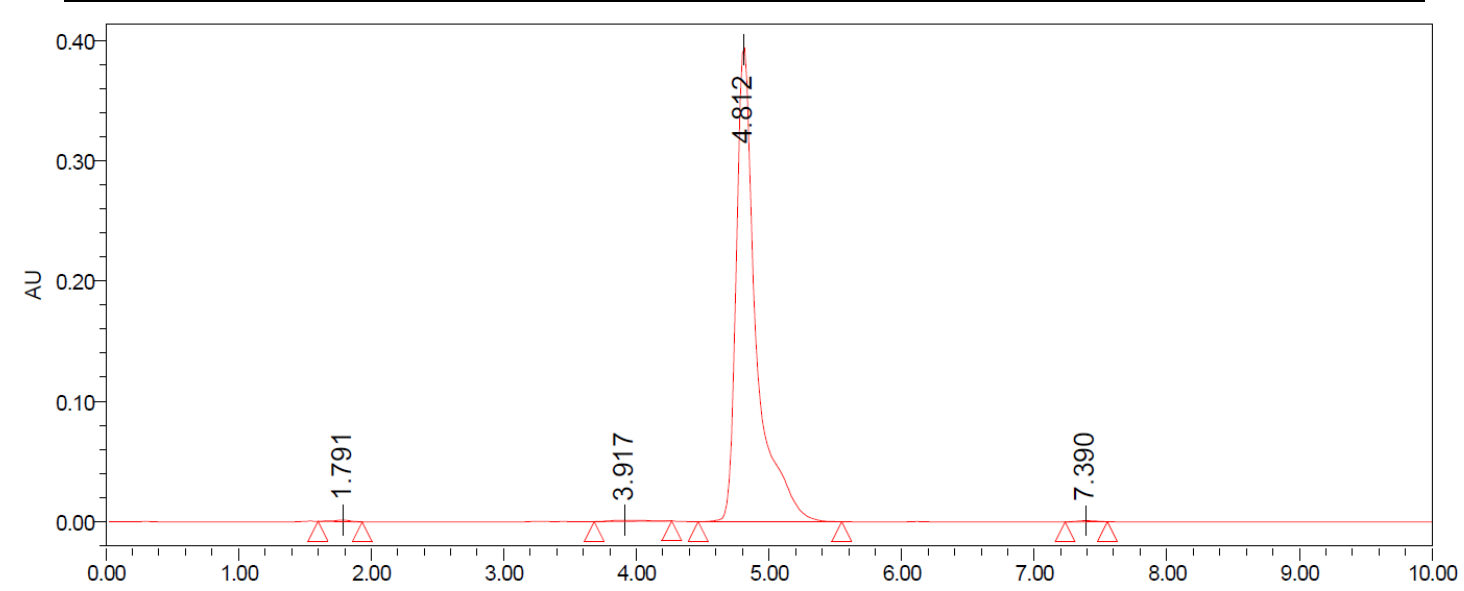

Figure S43. HPLC spectrum of compound $\mathbf{4 7}$

\begin{tabular}{|c|c|c|c|}
\hline Instrument: & Waters e2695 & Column: & $\begin{array}{c}\text { Phenomenex column, C-18, } \\
5.0 \mu \mathrm{m}, 4.6 * 250 \mathrm{~mm}\end{array}$ \\
\hline Mobile Phase: & $\begin{array}{c}\text { Phase A: water } \\
\text { Phase B: acetonitrile; } \\
\text { A/ B }=45 / 55, \mathrm{v} / \mathrm{v}\end{array}$ & Flow rate: & $1.0 \mathrm{~mL} / \mathrm{min}$ \\
\hline Ret. Time (min): & 4.021 & Purity $(\%)$ & 98.55 \\
\hline
\end{tabular}

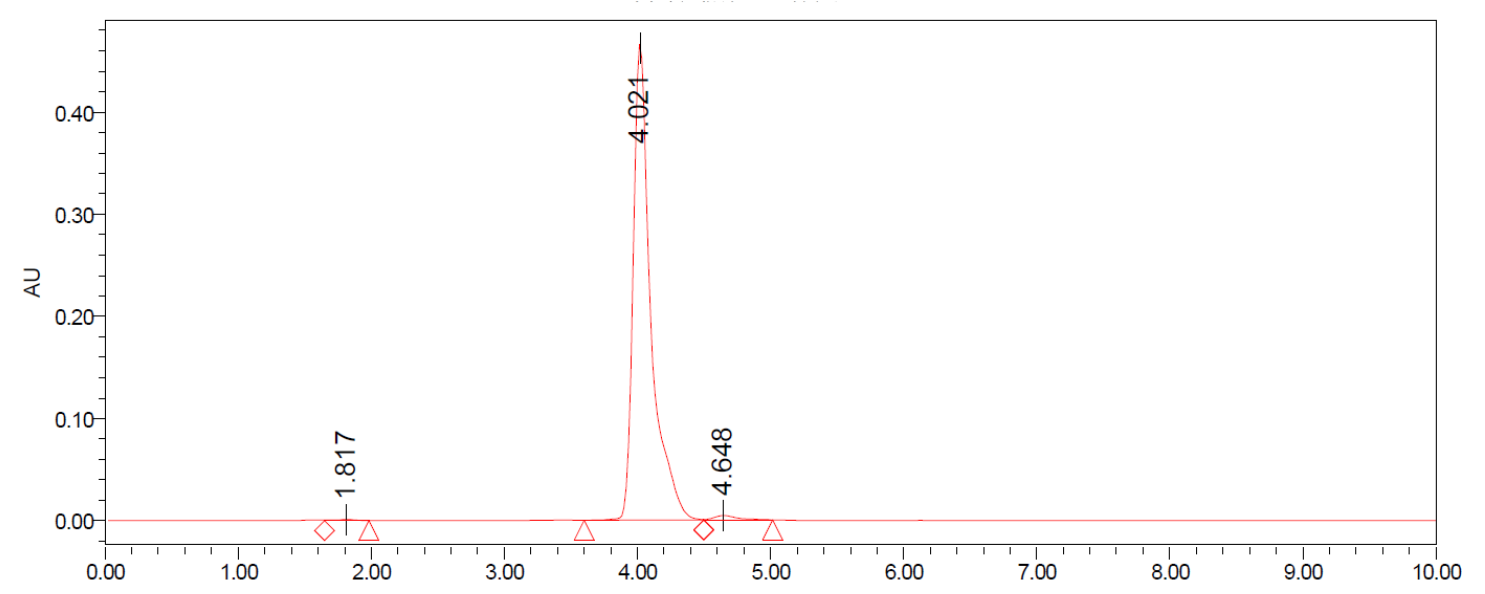

Figure S44. HPLC spectrum of compound $\mathbf{4 8}$ 


\begin{tabular}{|c|c|c|c|}
\hline Instrument: & Waters e2695 & Column: & $\begin{array}{c}\text { Phenomenex column, C-18, } \\
5.0 \mu \mathrm{m}, 4.6 * 250 \mathrm{~mm}\end{array}$ \\
\hline Mobile Phase: & $\begin{array}{c}\text { Phase A: water } \\
\text { Phase B: acetonitrile; } \\
\mathrm{A} / \mathrm{B}=45 / 55, \mathrm{v} / \mathrm{v}\end{array}$ & Flow rate: & $1.0 \mathrm{~mL} / \mathrm{min}$ \\
\hline Ret. Time (min): & 4.782 & Purity $(\%)$ & 99.64 \\
\hline
\end{tabular}

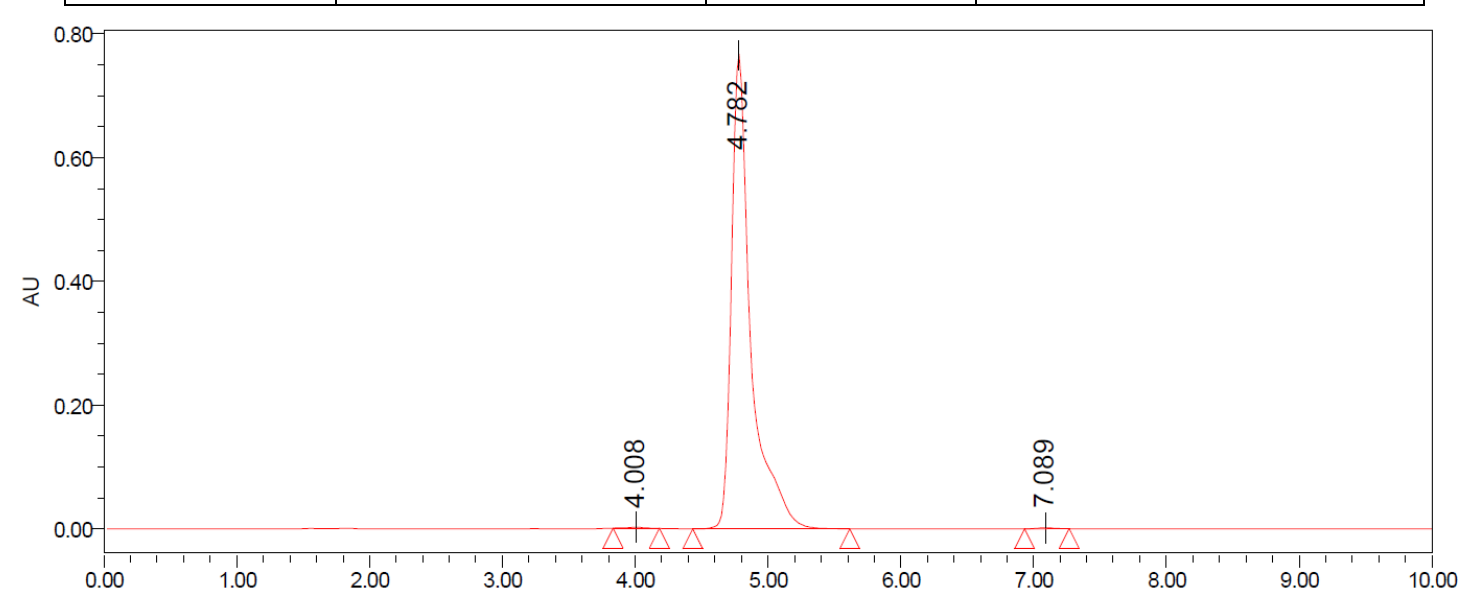

Figure S45. HPLC spectrum of compound 49

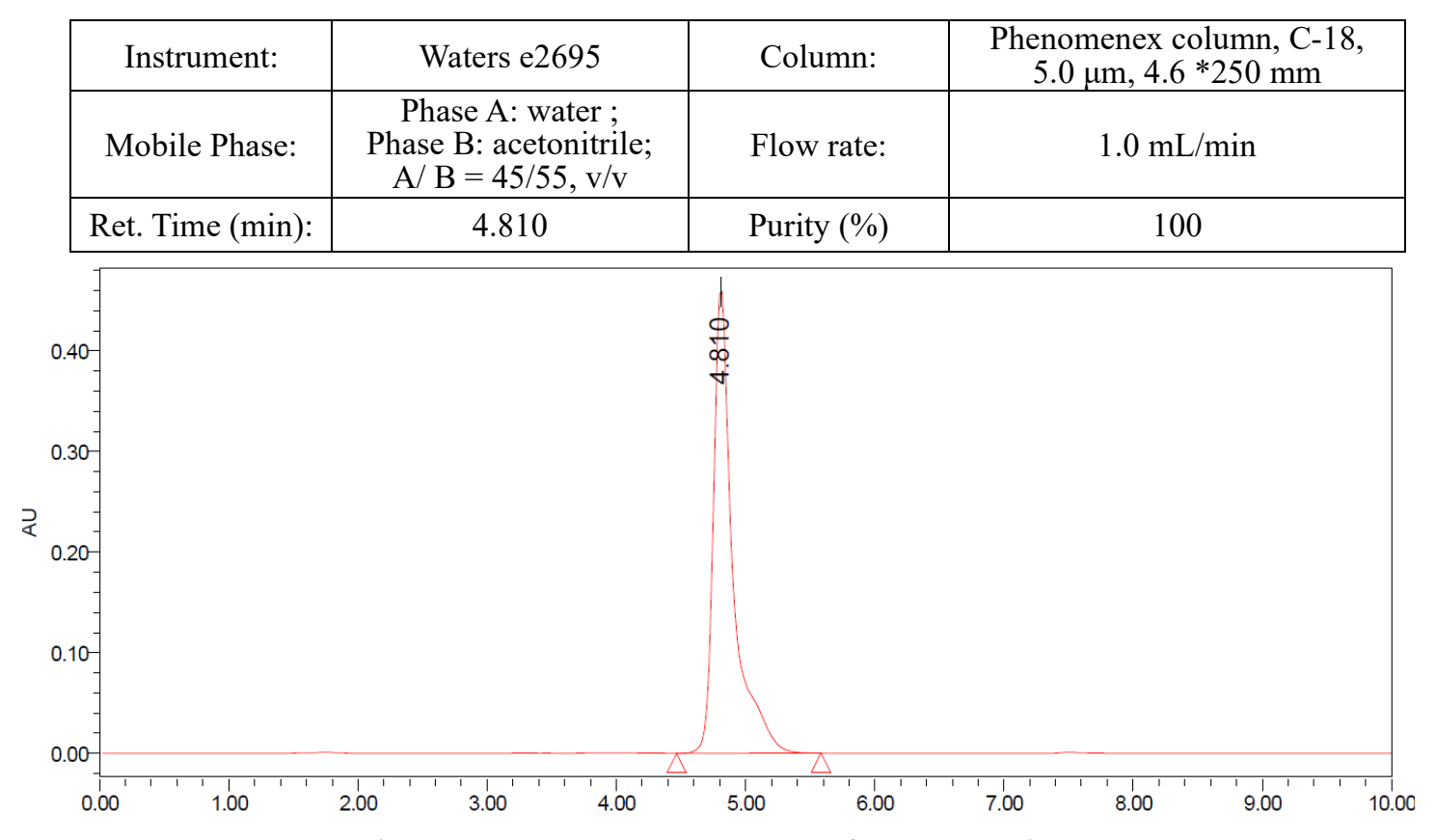

Figure S46. HPLC spectrum of compound $\mathbf{5 0}$ 


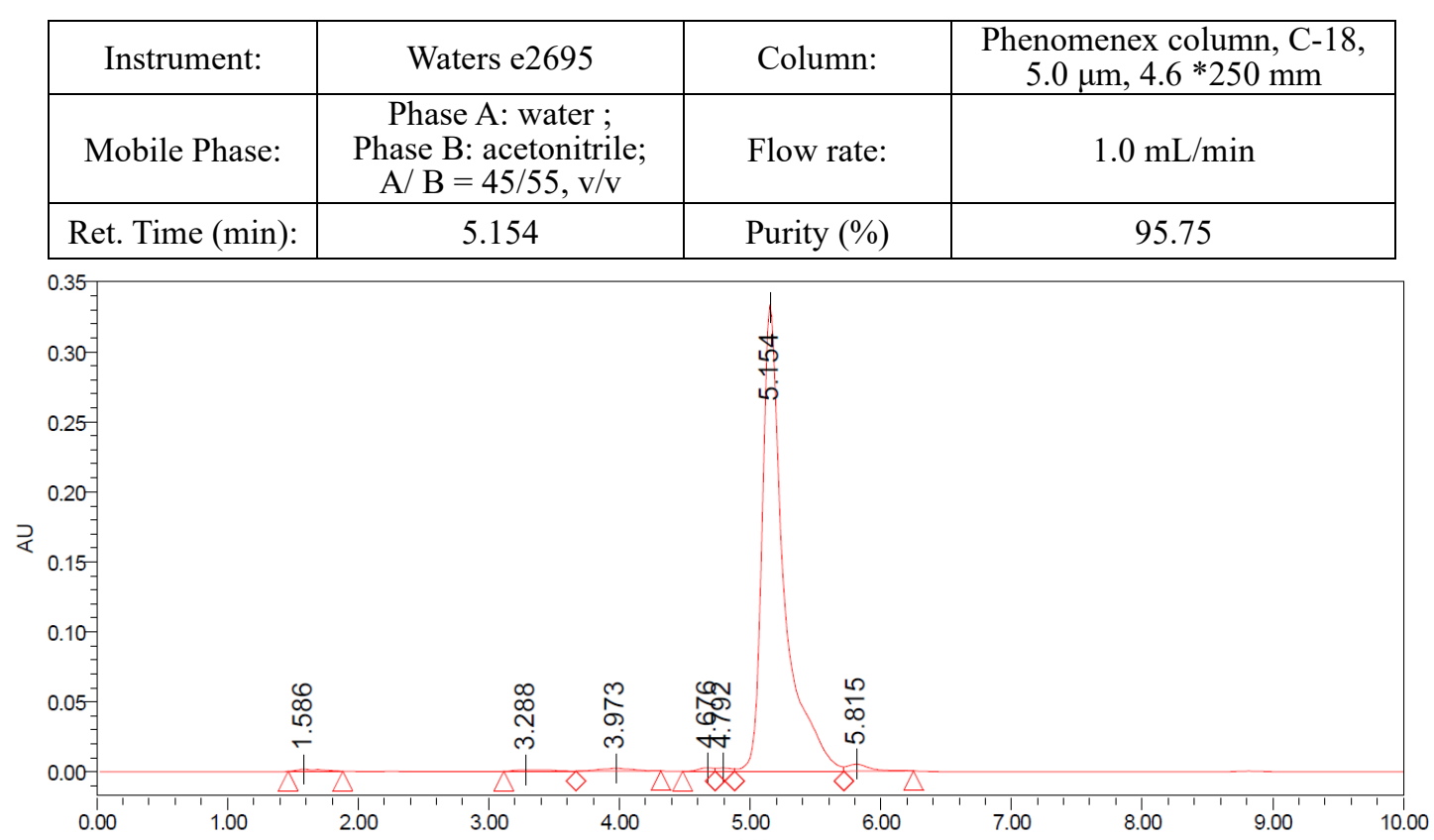

Figure S47. HPLC spectrum of compound $\mathbf{5 1}$

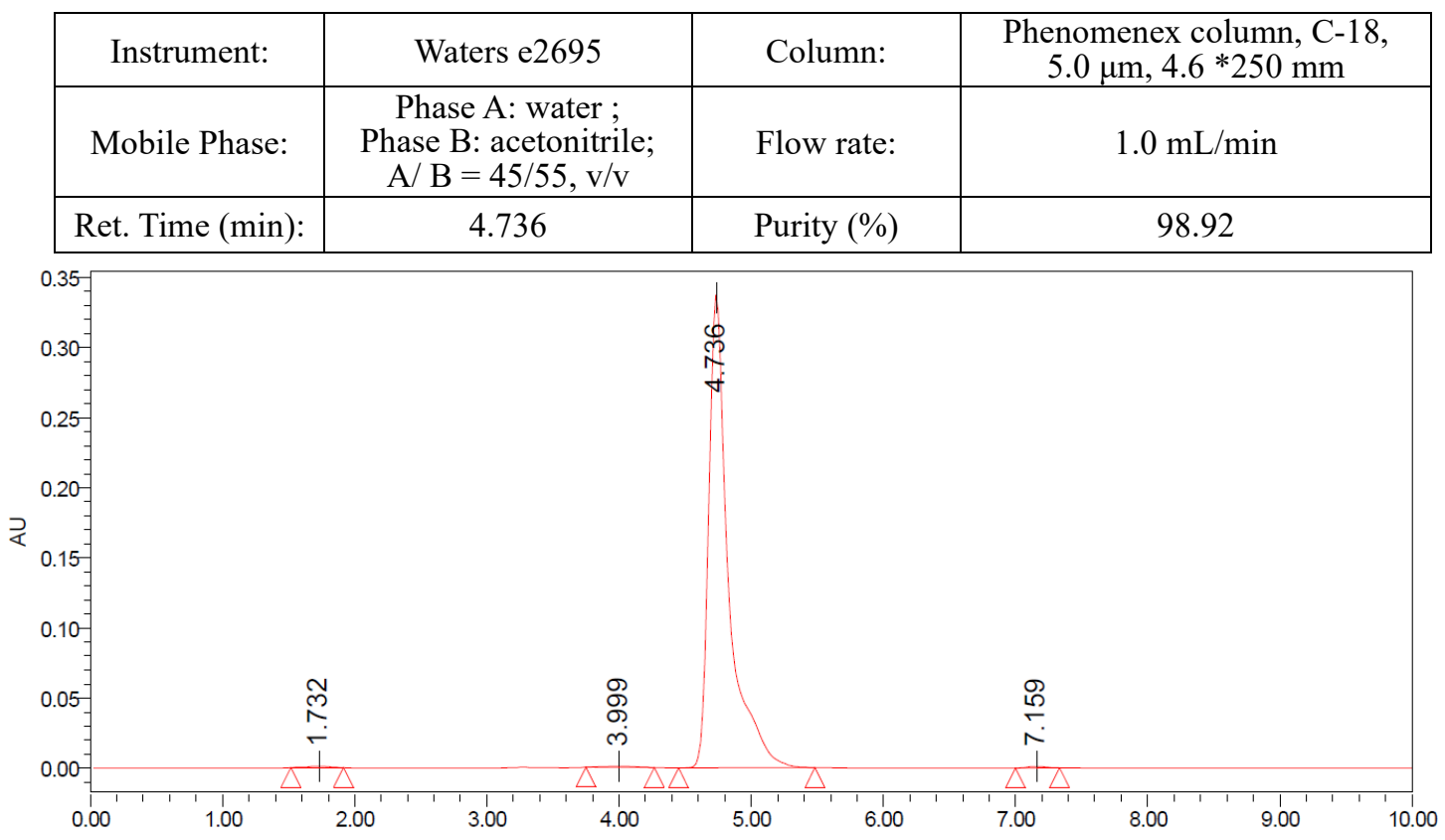

Figure S48. HPLC spectrum of compound $\mathbf{5 2}$ 


\begin{tabular}{|c|c|c|c|}
\hline Instrument: & Waters e2695 & Column: & $\begin{array}{c}\text { Phenomenex column, C-18, } \\
5.0 \mu \mathrm{m}, 4.6 * 250 \mathrm{~mm}\end{array}$ \\
\hline Mobile Phase: & $\begin{array}{c}\text { Phase A: water; } \\
\text { Phase B: acetonitrile; } \\
\text { A/ B = 45/55, v/v }\end{array}$ & Flow rate: & $1.0 \mathrm{~mL} / \mathrm{min}$ \\
\hline Ret. Time (min): & 5.038 & Purity (\%) & 97.52 \\
\hline
\end{tabular}

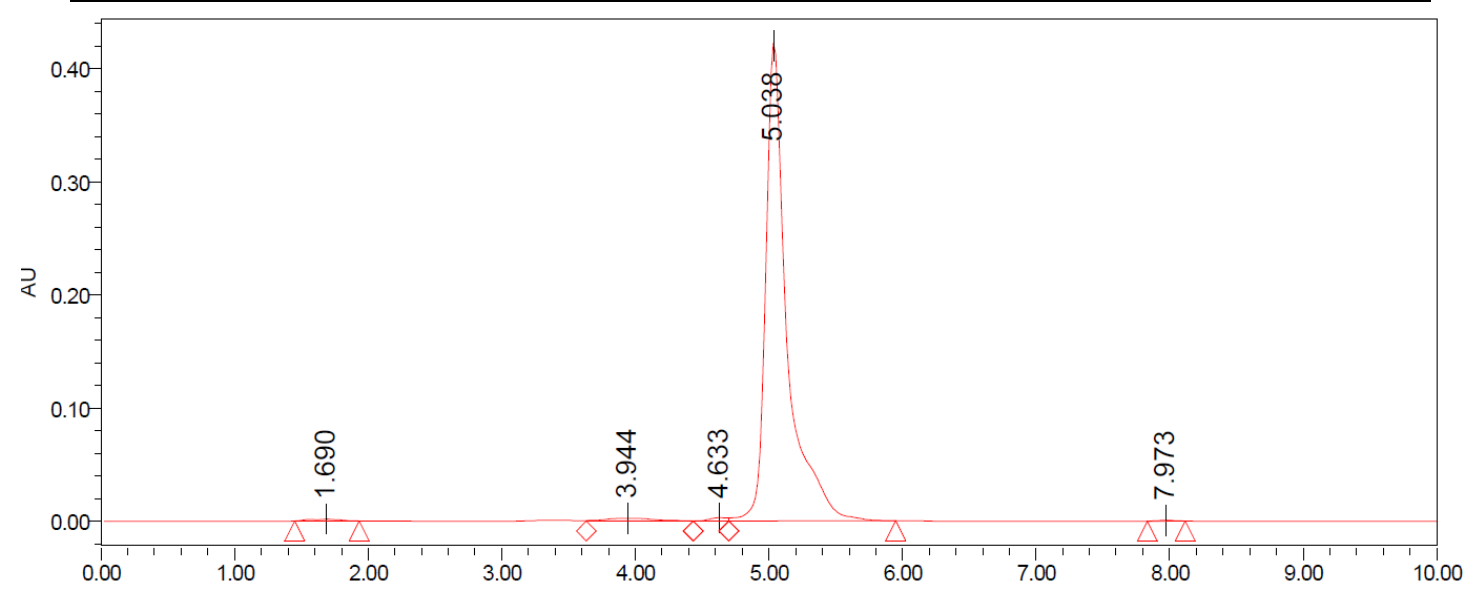

Figure S49. HPLC spectrum of compound $\mathbf{5 3}$

\begin{tabular}{|c|c|c|c|}
\hline Instrument: & Waters e2695 & Column: & $\begin{array}{c}\text { Phenomenex column, C-18, } \\
5.0 \mu \mathrm{m}, 4.6 * 250 \mathrm{~mm}\end{array}$ \\
\hline Mobile Phase: & $\begin{array}{c}\text { Phase A: water; } \\
\text { Phase B: acetonitrile; } \\
\text { A B = 45/55, v/v }\end{array}$ & Flow rate: & $1.0 \mathrm{~mL} / \mathrm{min}$ \\
\hline Ret. Time (min): & 4.749 & Purity (\%) & 98.20 \\
\hline
\end{tabular}

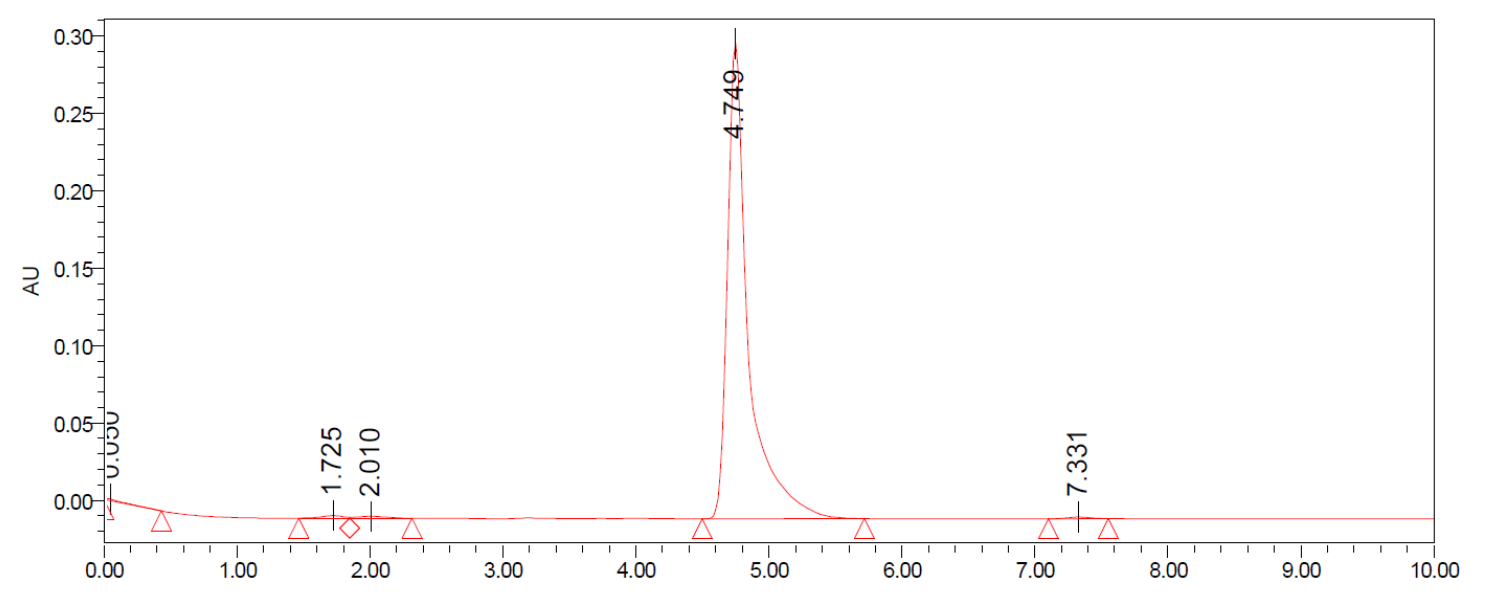

Figure S50. HPLC spectrum of compound $\mathbf{5 4}$ 


\begin{tabular}{|c|c|c|c|}
\hline Instrument: & Waters e2695 & Column: & $\begin{array}{c}\text { Phenomenex column, C-18, } \\
5.0 \mu \mathrm{m}, 4.6 * 250 \mathrm{~mm}\end{array}$ \\
\hline Mobile Phase: & $\begin{array}{c}\text { Phase A: water } \\
\text { Phase B: acetonitrile; } \\
\text { A/ B = 45/55, v/v }\end{array}$ & Flow rate: & $1.0 \mathrm{~mL} / \mathrm{min}$ \\
\hline Ret. Time (min): & 6.370 & Purity (\%) & 97.87 \\
\hline
\end{tabular}

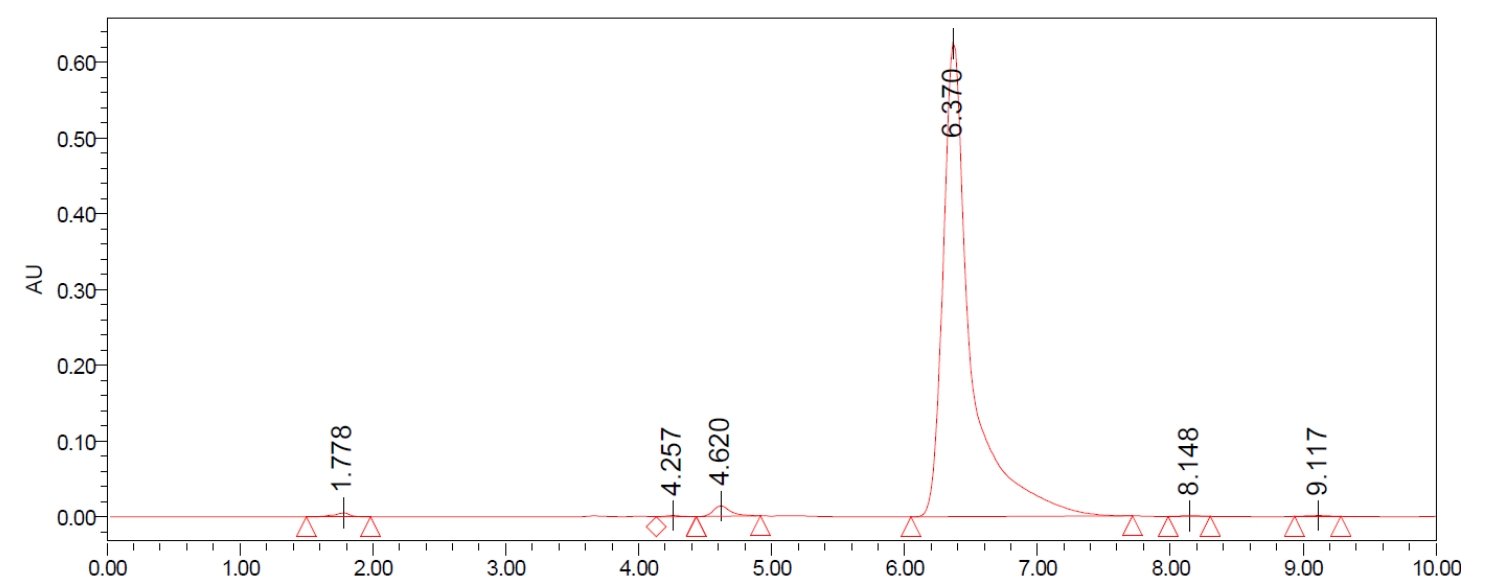

Figure S51. HPLC spectrum of compound $\mathbf{5 5}$ 